University of Tennessee Health Science Center

UTHSC Digital Commons

$12-2008$

\title{
Investigation of the Vesicular Stomatitis Virus Matrix Protein: Uncoating and Assembly
}

Chad E. Mire

University of Tennessee Health Science Center

Follow this and additional works at: https://dc.uthsc.edu/dissertations

Part of the Viruses Commons

\section{Recommended Citation}

Mire, Chad E. , "Investigation of the Vesicular Stomatitis Virus Matrix Protein: Uncoating and Assembly" (2008). Theses and Dissertations (ETD). Paper 185. http://dx.doi.org/10.21007/etd.cghs.2008.0213.

This Dissertation is brought to you for free and open access by the College of Graduate Health Sciences at UTHSC Digital Commons. It has been accepted for inclusion in Theses and Dissertations (ETD) by an authorized administrator of UTHSC Digital Commons. For more information, please contact jwelch30@uthsc.edu. 


\title{
Investigation of the Vesicular Stomatitis Virus Matrix Protein: Uncoating and Assembly
}

\author{
Abstract \\ Vesicular Stomatitis Virus (VSV) is a simple, enveloped, nonsegmented negative-strand RNA virus and is \\ the prototype rhabdovirus to study viral entry, transcription, replication, and assembly. The matrix protein \\ (M) of VSV is a central component of the viral replication cycle. While being the smallest of the viral \\ proteins it is multifunctional and is involved in uncoating, cytopathic effects (CPE), and assembly of the \\ virus. $M$ protein interactions involved in the uncoating and assembly of VSV have been examined in this \\ dissertation. \\ Uncoating of VSV involves dissociation of M from the ribonucleoprotein core (RNPs) of the virus. Current \\ models of VSV uncoating propose that following membrane fusion M protein is released from the RNP \\ with subsequent diffusion of $M$ into the cytoplasm and distribution of some of the released $M$ to the \\ nucleus of a host cell. The studies in Chapter 2 investigated where in the endocytic pathway uncoating \\ occurs, where $M$ is located following uncoating, and the role of the cytoskeleton in distribution of input $M$ \\ by using a VSV, containing fluorescent M protein (rVSV-M-Lumio-Green). I found that uncoating occurs \\ primarily in early endosomes and results in the majority of $M$ remaining associated with the endosomal \\ membrane which eventually localizes to the perinuclear recycling endosomes. A small fraction of $M$, \\ which is presumably released into the cytosol, gets delivered to the nuclear envelope, and I found that the \\ typical polymerized actin or microtubules within host cells were not required for distribution of $\mathrm{M}$ to the \\ nuclear envelope.
}

Uncoating and assembly of the VSV genome occurs on membranes within the cytoplasm of the host cell. Exactly how both of these processes can occur in the same environment (e.g. the cytoplasmically exposed membrane surface) without modification of $\mathrm{M}$ protein by phosphorylation, cleavage, or some other change has been an intriguing question in the field. In Chapter 3 I present results showing a pH effect on the M-Lumio-Green protein fluorescence in vitro and during the endocytosis of virions which was dependent on $\mathrm{G}$ protein. I also observed that low pH enhanced the release of M from rVSV-wt virions, which suggested that acidification of the virus interior results in the dissociation of $\mathrm{M}$ contacts within the virus enhancing the uncoating process.

An exposed protease-sensitive loop located between amino acids 120 to 129 of $\mathrm{M}$ has been shown to be important for $\mathrm{M}$ protein self-association and has been proposed to be crucial for assembly of virions. This knowledge comes from protease treated, purified $M$ protein and not from mutagenesis studies. In Chapter $4 \mathrm{I}$ examined mutations in the exposed loop and in particular a conserved aspartate at residue 125 of a conserved LXD sequence. I found that virions with mutations at residue 123 or 125 of the LXD motif have two phenotypes; 1 ) an assembly defect and 2) reduced viral protein synthesis starting at 4 hours post infection. These two phenotypes have not been separated genetically and the LXD motif may represent a motif of M involved in assembly and support of VSV protein translation. Vesicular Stomatitis Virus (VSV) is a simple, enveloped, nonsegmented negative-strand RNA virus and is the prototype rhabdovirus to study viral entry, transcription, replication, and assembly. The matrix protein (M) of VSV is a central component of the viral replication cycle. While being the smallest of the viral proteins it is multifunctional and is involved in uncoating, cytopathic effects (CPE), and assembly of the virus. M protein interactions involved in the uncoating and assembly of VSV have been examined in this dissertation.

\section{Document Type}

Dissertation 


\section{Degree Name}

Doctor of Philosophy (PhD)

\section{Program}

Molecular Sciences

\section{Research Advisor}

Michael A. Whitt, Ph.D.

\section{Keywords}

Vesicular Stomatitis Virus, VSV, matrix, M, uncoating, entry, trafficking, assembly, translation, LXD motif

\section{Subject Categories}

Medicine and Health Sciences | Organisms | Viruses 


\title{
Investigation of the Vesicular Stomatitis Virus Matrix Protein: Uncoating and Assembly
}

\author{
A Dissertation \\ Presented for \\ The Graduate Studies Council \\ The University of Tennessee \\ Health Science Center
}

\author{
In Partial Fulfillment \\ Of the Requirements for the Degree \\ Doctor of Philosophy \\ From The University of Tennessee
}

By

Chad Edward Mire

December 2008 
Copyright (C) 2008 by Chad Edward Mire All rights reserved 


\section{Dedication}

This dissertation is dedicated to my wife, Mrs. Erika Mire and to my parents, Mr. Carl Mire and Mrs. Connie Mire for all of their support and belief in me throughout my life. 


\section{Acknowledgments}

I would like to thank my advisor, Michael A. Whitt, Ph. D., for giving me the opportunity to work in his lab and for his excellent training. I have appreciated his patience and guidance in preparing me for my career in science. I would also like to thank my committee members Robert Belland, Ph.D., John Cox, Ph.D., Lawrence Pfeffer, Ph.D., and Patrick Ryan, Ph.D. for their input and suggestions during my graduate studies.

I would be remiss if I did not acknowledge past and present members of the Whitt lab for their interaction and support over the course of my graduate school experience. Carolyn Matthews, Hideki Tani, Ph.D., Makiko Wantanabe, Ph.D., Elizabeth Matheny, Himangi Jayakar, Ph.D., and Erika Dillard are all those that I would like to thank for making the Whitt lab a positive experience for me.

I would also like to thank Scot Ouellette, Ph.D. and Tony Marion, Ph.D. for their interactions with me. I appreciated the ability to talk about life in general with them but also being able to talk about all realms of science and our ideas and opinions about them. 


\begin{abstract}
Vesicular Stomatitis Virus (VSV) is a simple, enveloped, nonsegmented negativestrand RNA virus and is the prototype rhabdovirus to study viral entry, transcription, replication, and assembly. The matrix protein (M) of VSV is a central component of the viral replication cycle. While being the smallest of the viral proteins it is multifunctional and is involved in uncoating, cytopathic effects (CPE), and assembly of the virus. M protein interactions involved in the uncoating and assembly of VSV have been examined in this dissertation.

Uncoating of VSV involves dissociation of M from the ribonucleoprotein core (RNPs) of the virus. Current models of VSV uncoating propose that following membrane fusion $\mathrm{M}$ protein is released from the RNP with subsequent diffusion of $\mathrm{M}$ into the cytoplasm and distribution of some of the released $\mathrm{M}$ to the nucleus of a host cell. The studies in Chapter 2 investigated where in the endocytic pathway uncoating occurs, where $\mathrm{M}$ is located following uncoating, and the role of the cytoskeleton in distribution of input M by using a VSV, containing fluorescent M protein (rVSV-MLumio-Green). I found that uncoating occurs primarily in early endosomes and results in the majority of $\mathrm{M}$ remaining associated with the endosomal membrane which eventually localizes to the perinuclear recycling endosomes. A small fraction of $\mathrm{M}$, which is presumably released into the cytosol, gets delivered to the nuclear envelope, and I found that the typical polymerized actin or microtubules within host cells were not required for distribution of $\mathrm{M}$ to the nuclear envelope.
\end{abstract}

Uncoating and assembly of the VSV genome occurs on membranes within the cytoplasm of the host cell. Exactly how both of these processes can occur in the same environment (e.g. the cytoplasmically exposed membrane surface) without modification of $\mathrm{M}$ protein by phosphorylation, cleavage, or some other change has been an intriguing question in the field. In Chapter 3 I present results showing a $\mathrm{pH}$ effect on the M-LumioGreen protein fluorescence in vitro and during the endocytosis of virions which was dependent on $\mathrm{G}$ protein. I also observed that low $\mathrm{pH}$ enhanced the release of $\mathrm{M}$ from rVSV-wt virions, which suggested that acidification of the virus interior results in the dissociation of $\mathrm{M}$ contacts within the virus enhancing the uncoating process.

An exposed protease-sensitive loop located between amino acids 120 to 129 of M has been shown to be important for $\mathrm{M}$ protein self-association and has been proposed to be crucial for assembly of virions. This knowledge comes from protease treated, purified $\mathrm{M}$ protein and not from mutagenesis studies. In Chapter 4 I examined mutations in the exposed loop and in particular a conserved aspartate at residue 125 of a conserved LXD sequence. I found that virions with mutations at residue 123 or 125 of the LXD motif have two phenotypes; 1) an assembly defect and 2) reduced viral protein synthesis starting at 4 hours post infection. These two phenotypes have not been separated genetically and the LXD motif may represent a motif of M involved in assembly and support of VSV protein translation. 


\section{Table of Contents}

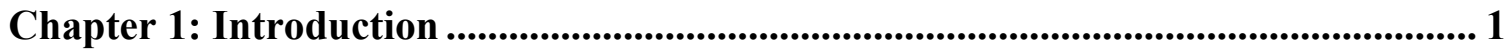

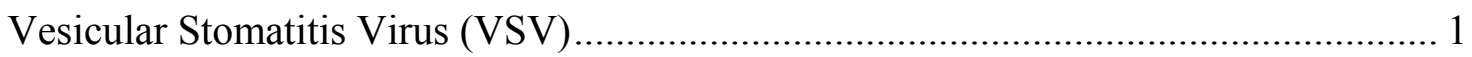

Vesicular stomatitis virus (VSV): the prototype Rhabdovirus..................................... 1

A brief history of VSV in the Americas................................................................ 1

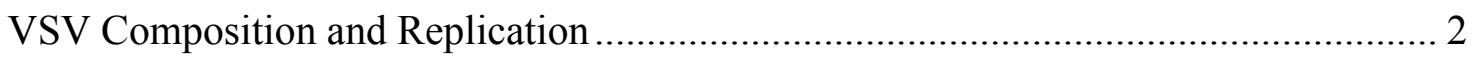

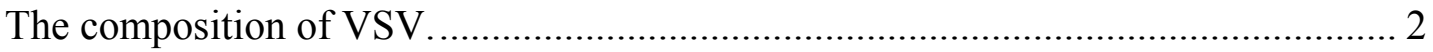

The viral replication cycle of VSV ....................................................................... 2

The VSV Matrix (M) Protein ......................................................................................... 4

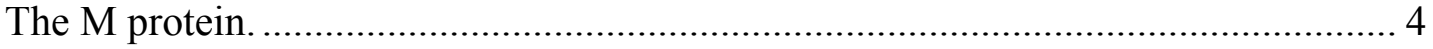

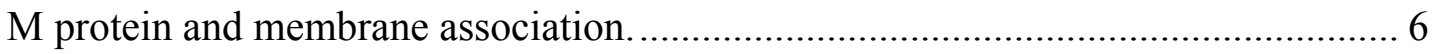

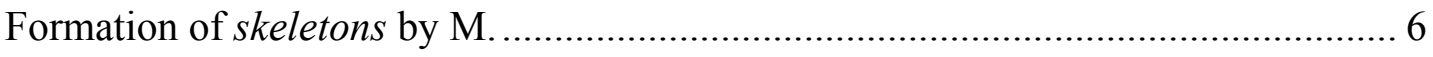

Membrane associated M protein and its association with the

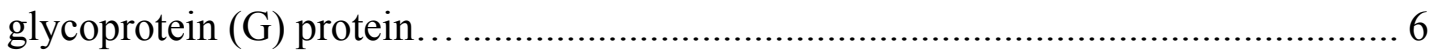

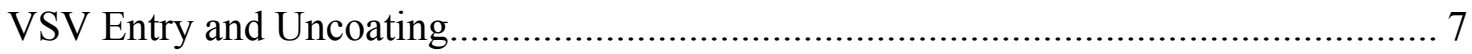

Entry of enveloped viruses into the host cell........................................................... 7

Uncoating of genomes after entry of enveloped viruses............................................ 8

VSV and the uncoating dilemma....................................................................... 8

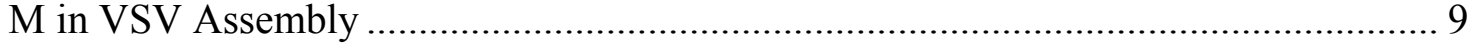

Budding of VSV progeny from host cells. ............................................................. 9

$\mathrm{M}$ protein and nucleocapsid condensation............................................................ 9

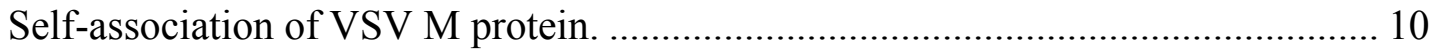

VSV Cytopathogenicity and Methionine 51 of M ................................................... 10

Leader RNA transcripts. ...................................................................................... 10

Inhibition of host gene expression by M protein.................................................. 10

Inhibition of nucleocytoplasmic transport by M protein: implications for

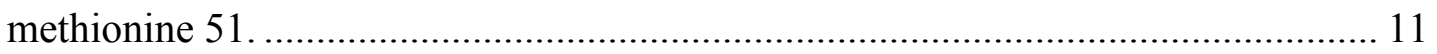

Cell rounding: as a hallmark of VSV infection. .................................................... 12

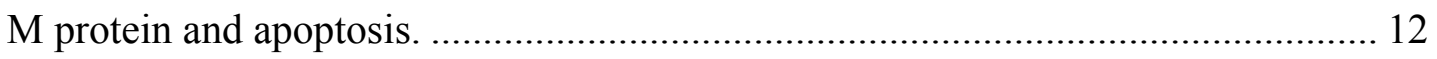

Enveloped RNA Viruses: Origins and M Proteins..................................................... 13 


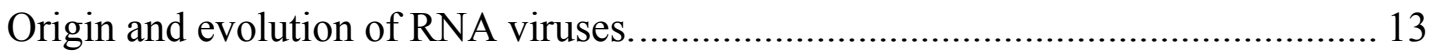

M proteins of enveloped RNA viruses............................................................... 13

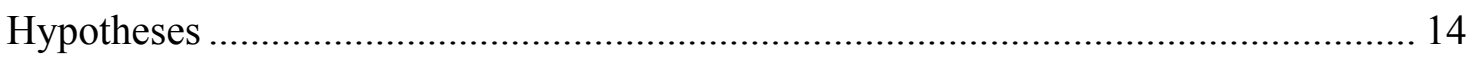

Hypothesis 1 . ............................................................................................... 14

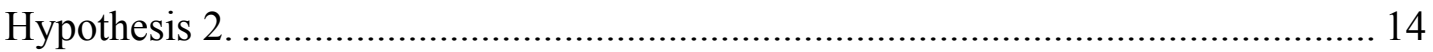

\section{Chapter 2: Analysis of Matrix Protein Distribution after}

Vesicular Stomatitis Virus Uncoating ....................................................... 16

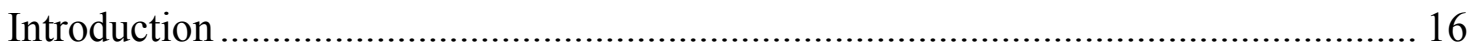

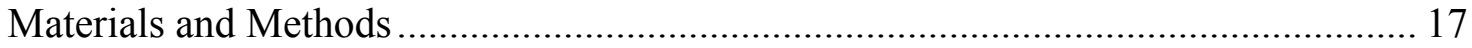

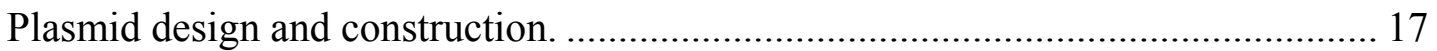

Generation of full-length VSV cDNAs encoding MFC, M-GST, M-Lumio,

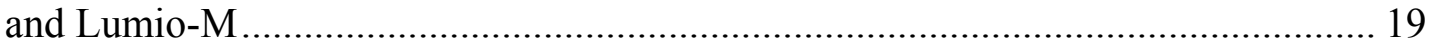

Recovery and characterization of recombinant VSV (rVSV)................................. 19

Transient expression of MFC, M-Lumio, and Lumio-M proteins. ............................ 19

Immunofluorescence (IF) staining and confocal microscopy................................... 20

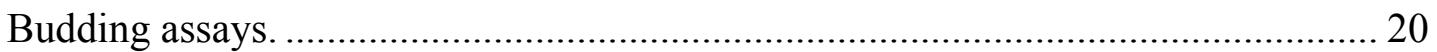

Immunoblot analysis of rVSV-wt and rVSV-M-MFC virions.................................. 21

Lumio $^{\mathrm{TM}}$ Green labeling and quantification of rVSV ............................................. 21

Live-cell synchronized entry assay......................................................................... 22

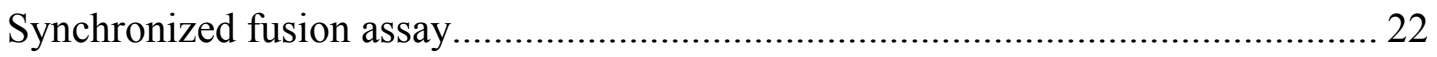

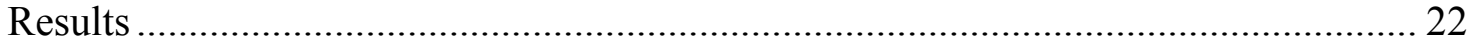

Recovery and characterization of recombinant VSV encoding fluorescent M

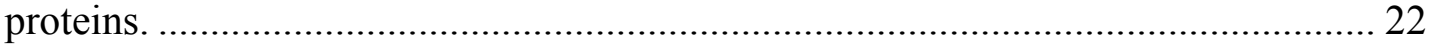

Fluorescent labeling of rVSV-M-Lumio ............................................................... 26

Live-cell entry of rVSV-M-Lumio-Green............................................................. 30

Synchronized fusion assay with rVSV-M-Lumio-Green........................................... 30

The perinuclear endosomes associated with M-Lumio-Green colocalize

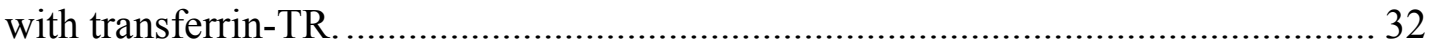

Trafficking of M-Lumio-Green to perinuclear endosomes and the nuclear envelope does not require actin or tubulin. 
Decrease in colocalization of M-Lumio-Green and N correlates with productive infection and does not require tubulin................................................. 36

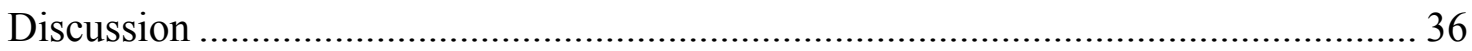

Chapter 3: Low pH Enhancement of Matrix Uncoating......................................... 44

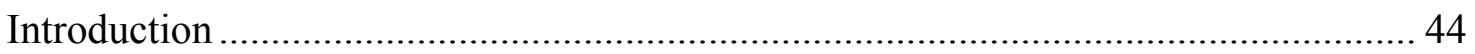

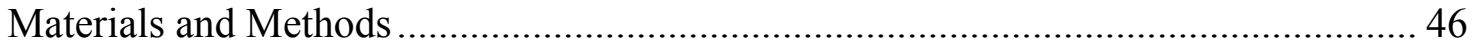

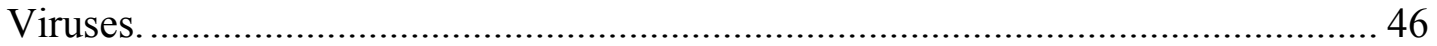

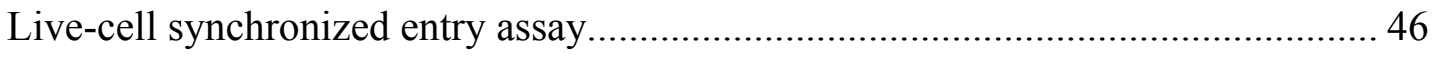

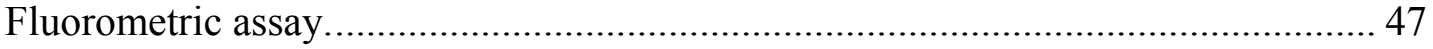

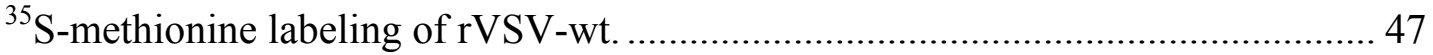

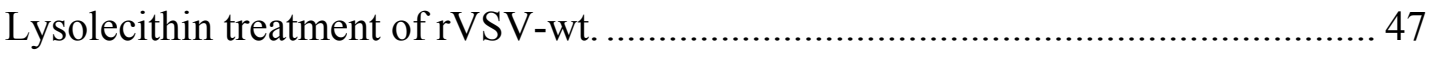

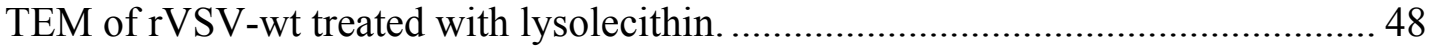

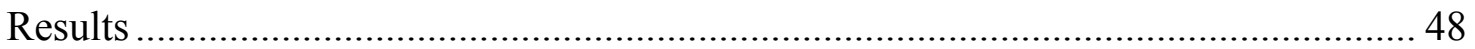

Loss of fluorescence intensity over time during rVSV entry.............................. 48

Loss of fluorescence intensity of rVSV-M-Lumio-Green in the

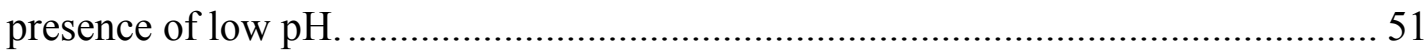

Fluorescence intensity loss of rVSV-M-Lumio-Green is G protein dependent. ....... 51

Low $\mathrm{pH}$ enhances release of $\mathrm{M}$ protein from VSV virions................................... 53

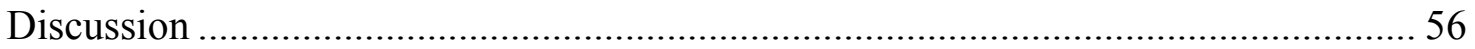

\section{Chapter 4: Examination of Mutations in the Exposed Loop of the}

VSV Matrix Protein ...........................................................................63

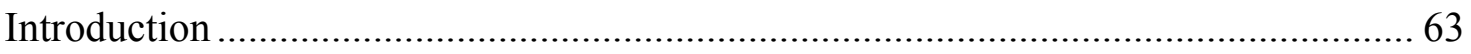

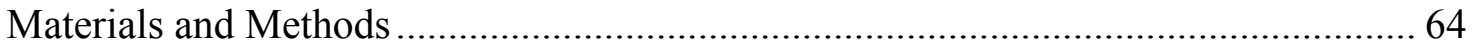

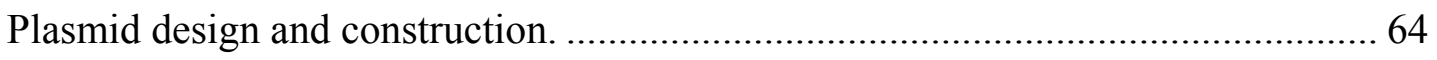

Generation of full-length VSV cDNAs encoding M loop mutants. ........................ 64

Recovery and characterization of recombinant VSV (rVSV). ............................... 64

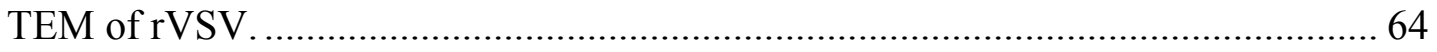

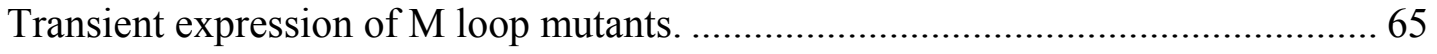

Immunofluorescence (IF) staining and confocal microscopy. .............................. 65 


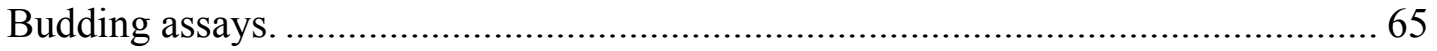

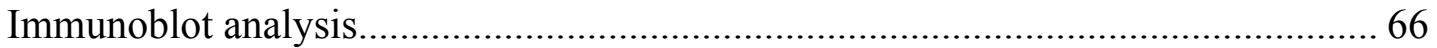

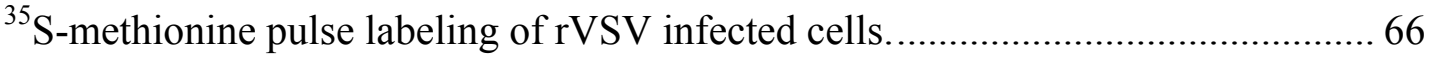

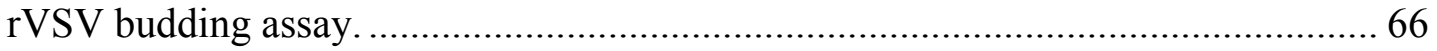

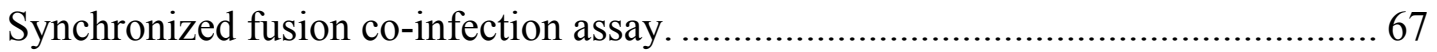

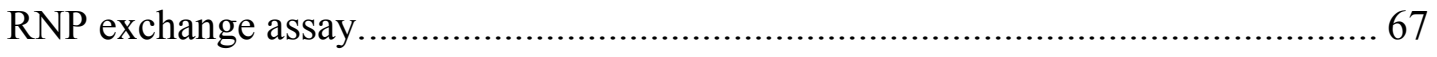

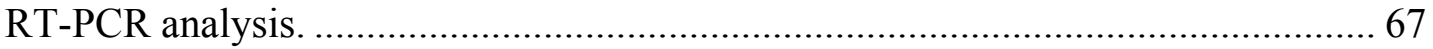

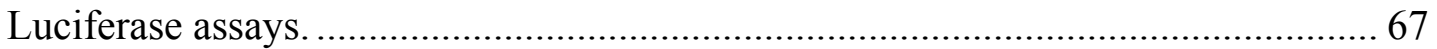

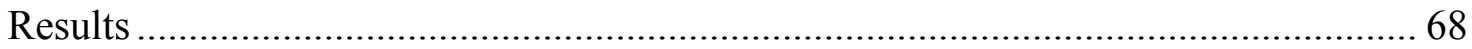

Recovery and characterization of recombinant (r)VSV with M loop mutations....... 68

rVSV M loop mutants show reduced viral protein synthesis at later times post

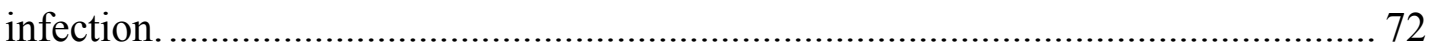

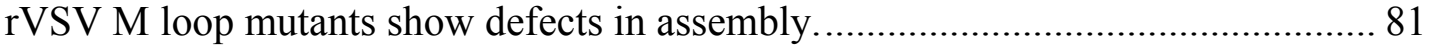

Reduction in viral protein synthesis is not due to classical host responses,

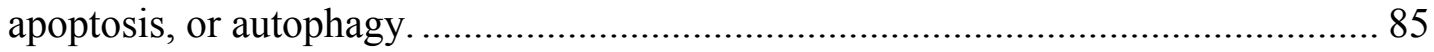

rVSV infection does not reduce cap-independent protein synthesis. ...................... 89

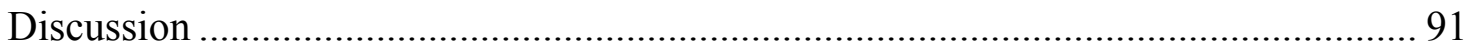

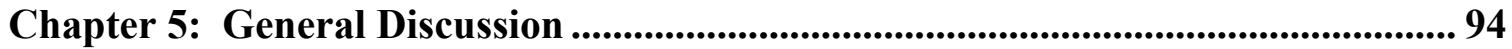

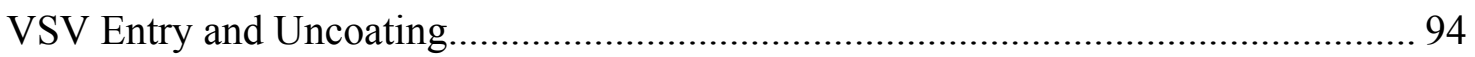

Upon entry, the VSV virion must be disassembled.............................................. 94

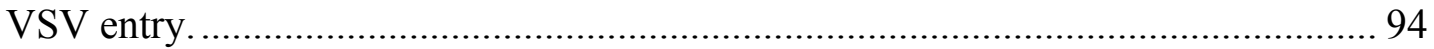

Acidification of the VSV interior and its effect on M....................................... 97

Completion of uncoating and the subsequent distribution of M. ........................... 97

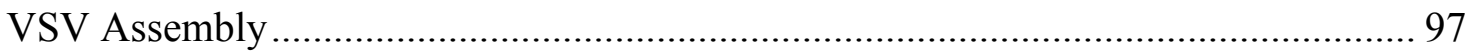

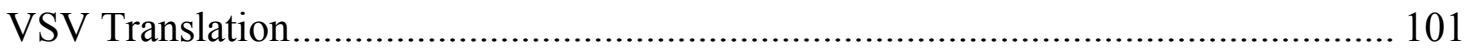

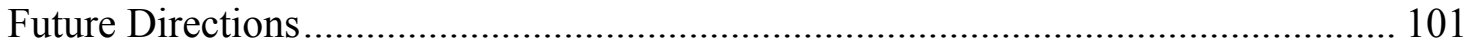

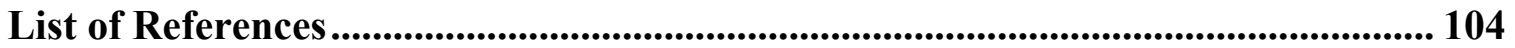

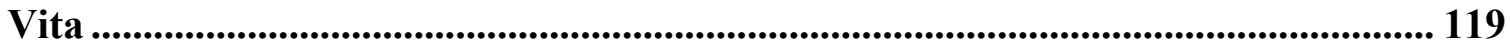




\section{List of Figures}

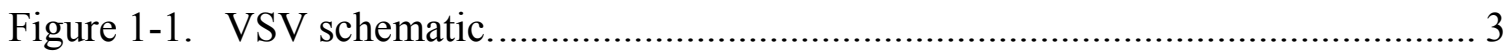

Figure 1-2. Crystal structure of the VSV M protein. ................................................. 5

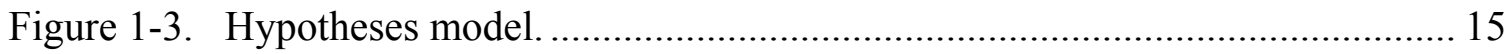

Figure 2-1. Schematic of fluorescent M proteins and rVSV genomes. ........................ 18

Figure 2-2. Characterization of the MFC protein and rVSV-M-MFC virions. .............. 24

Figure 2-3. Characterization of Lumio-M and M-Lumio and rVSV-M-Lumio/(Lumio-M) virions................................................. 27

Figure 2-4. Labeling of rVSV-M-Lumio with Lumio ${ }^{\mathrm{TM}}$ Green. ................................... 29

Figure 2-5. Synchronized entry assay with rVSV-M-Lumio-Green ........................... 31

Figure 2-6. Synchronized fusion assay with rVSV-M-Lumio-Green........................... 33

Figure 2-7. Identification of perinuclear structures associated with M-Lumio-Green post fusion. ............................................................ 34

Figure 2-8. Distribution of M-Lumio-Green in the presence of cytochalasin D........... 37

Figure 2-9. Distribution of M-Lumio-Green in the presence of nocodazole................. 38

Figure 2-10. Release of RNPs from M-Lumio endosomes and productive infection in the presence of nocodazole................................... 39

Figure 3-1. $\Delta \mathrm{G}$ and rVSV-M-Lumio-Green fluorescence intensity during entry.......... 49

Figure 3-2. $\mathrm{pH}$ - and G-dependence of fluorescence intensity loss. ............................. 52

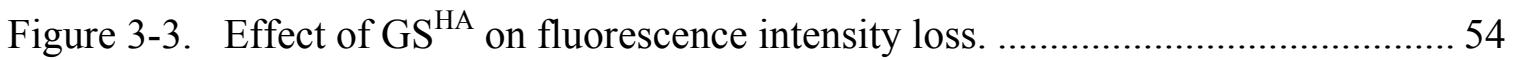

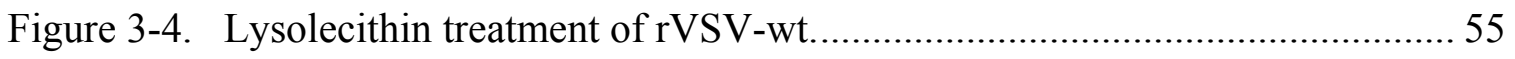

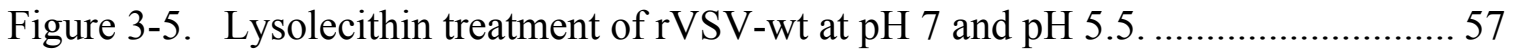

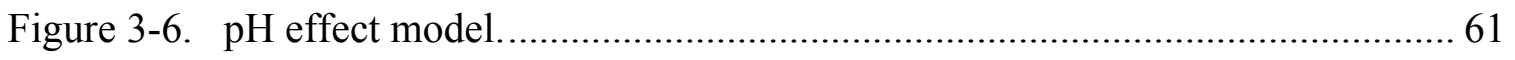

Figure 4-1. Design and characterization of the M loop mutant proteins. ...................... 69 
Figure 4-2. M loop sequence alignments.

Figure 4-3. Cell rounding of rVSV-wt and rVSV-D125A infected cells. .................... 73

Figure 4-4. Characterization of rVSV with M loop mutations. ................................ 75

Figure 4-5. ${ }^{35}$ S-methionine metabolic labeling of rVSV infected cells....................... 76

Figure 4-6. Time course of viral protein synthesis by

${ }^{35}$ S-methionine metabolic labeling................................................. 78

Figure 4-7. Quantitative real-time RT-PCR of virus genomes and mRNA during rVSV infection.................................................... 80

Figure 4-8. ${ }^{35}$ S-methionine metabolic labeling of cells co-infected with rVSV .......... 82

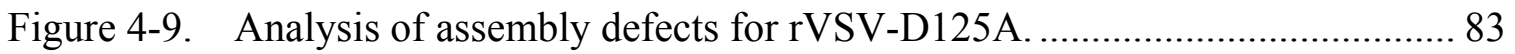

Figure 4-10. Analysis of PKR and RNaseL activation in rVSV-D125A infected cells. . 86

Figure 4-11. Analysis of reduced protein synthesis and host cell

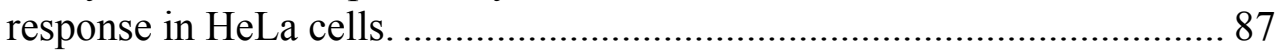

Figure 4-12. EMCV/IRES expression of luciferase in rVSV infected BSR cells. .......... 90

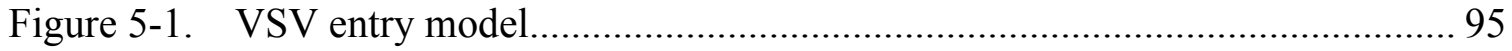

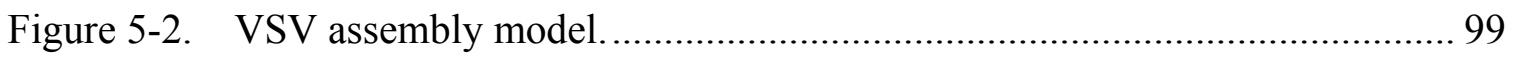

Figure 5-3. VSV translational support model for M........................................... 102 


\section{Chapter 1: Introduction}

\section{Vesicular Stomatitis Virus (VSV)}

Vesicular stomatitis virus (VSV): the prototype Rhabdovirus. VSV, is a simple, enveloped, nonsegmented, negative-strand RNA virus in the family Rhabdoviridae, and the order Mononegavirales. The Paramyxoviridae, Bornaviridae, and Filoviridae families complete the order Mononegavirales. Virions from these four families have a similar genome organization and share some properties of their transcription, replication, and assembly.

The Rhabdoviridae family includes three genera: the Lyssavirus genus, which includes rabies and the rabies-like viruses, the Emphemerovirus genus, which includes bovine ephemeral fever virus (BEFV), and the Vesiculovirus genus, which includes VSV. $\mathrm{VSV}$, is the prototype virus from the Rhabdoviridae family, and has been a platform of study for viral entry, transcription, replication, and assembly for many years. This is a direct result of its simple genome, broad cell tropism, and ability to grow to high titers in cell culture. There are two main serotypes of VSV found in the Western Hemisphere: the Indiana and New Jersey serotypes. Outside of the laboratory, mammals, mosquitoes, mites, and sand flies are among the hosts that can be infected by both serotypes. Of particular importance is the economic impact of the disease it causes, vesiculo stomatitis, in cattle, swine, and horses (121). This disease is characterized by vesicular lesions on the hooves, teats, and gums of infected animals, which are similar characteristics to foot and mouth disease. Though rare, VSV infections of humans can occur by accidental exposure in the laboratory or contact with infected animals in rural areas (56). Infection can result in flu-like symptoms for a few days, with vesicular lesions of the mouth, or no symptoms at all.

While not a human pathogen, VSV is a member of an order with many important human pathogens such as rabies virus, Ebola virus, Marburg virus, human parainfluenza virus, respiratory syncytial virus, and measles virus. Because virions found in the Mononegavirales share some aspects of their viral replication cycles, further insight into VSV's viral cycle can open a window to important information about the viral cycles of the human pathogens in this order. In addition to being a model, recombinant VSVs (rVSVs) are being tested as vaccine vectors and viral cytolytic vectors to fight certain cancers and HIV infections $(185,193)$. Developing a clearer understanding of VSV's viral cycle could help make these vectors more effective and safe for use in humans.

A brief history of VSV in the Americas. The two main serotypes of VSV found in the United States, the Indiana and New Jersey, were isolated in the 1920s. In 1925 a shipment of cattle that arrived in Richmond, Indiana from Missouri developed vesicular lesions in the mouth and were associated with an outbreak of vesicular stomatitis in the area. Virions isolated from the lesions were able to infect horses and could be serially 
passaged in animals. This virus then became the VS-Indiana strain (39). An outbreak of vesicular stomatitis occurred among cattle in New Jersey during the year of 1926. The agent responsible for this disease was found to be filterable and that it could infect cattle, guinea pigs, and horses. However, it was found that this virus was serologically different from the Indiana strain and became known as the VSV-New Jersey strain $(38,39)$. The Indiana and New Jersey serotypes have been the most commonly isolated strains in the Americas. In the United States, the last Indiana outbreak occurred in 1965 (96), whereas New Jersey was responsible for outbreaks in the United States in 1944, 1949, 1957, 1959, $1963,1982-83,1985,1995,1997,1998$, and 2005 that were mainly located in the west $(1,19,143,144)$. However, there is an island (Ossabaw) off of the coast of Georgia that is the only known place to have VSV-New Jersey as an enzootic disease where it is believed that a sand fly (Lutzomyia shannoni) on the island may be the vector that passes the virus to the feral pigs, horses, deer, cattle and raccoons that inhabit it (191). Besides the United States, VSV can also be found in other parts of the Americas including southern Mexico, Central America, and northern South America where sporadic epidemics emerge from the tropical areas (121).

\section{VSV Composition and Replication}

The composition of VSV. VSV is composed of an $11 \mathrm{~Kb}$, negative-strand RNA genome that contains five genes ( $\left.3^{\prime}-\mathrm{N}-\mathrm{P}-\mathrm{M}-\mathrm{G}-\mathrm{L}-5^{\prime}\right)$. These genes encode the nucleocapsid protein $(\mathrm{N})$, the phosphoprotein $(\mathrm{P})$, the matrix protein $(\mathrm{M})$, the glycoprotein (G), and the large polymerase protein (L) (Fig. 1-1). The virion's structure is composed of a helical nucleocapsid, containing L-P-N-RNA-M, surrounded by a membrane derived from an infected host cell membrane which has transmembrane trimeric $\mathrm{G}$ proteins protruding from it. These bullet-shaped VSV particles are an average of $180 \mathrm{~nm}$ long and $75 \mathrm{~nm}$ wide with around 400 trimeric $\mathrm{G}$ protein spikes on the exterior of the viral membrane and about 1,800 molecules of $\mathrm{M}$ protein between the nucleocapsid core and the viral membrane. The RNA genome is encapsidated by N protein with around 1,200 molecules of this protein tightly complexed to the RNA like beads along a string. The RNA-dependent RNA polymerase, consisting of the $\mathrm{L}$ and $\mathrm{P}$ proteins, is associated with this ribonucleoprotein complex and these proteins are present at 50 and 500 molecules per virion, respectively. This L-P-N-RNA complex makes up the ribonucleoprotein particle (RNP) which is coiled into a tightly packed helix of around 35 turns inside the viral membrane. The M protein condenses the RNP into this tightly packed helix, which, when condensed as described, is referred to as the skeleton.

The viral replication cycle of VSV. VSV takes advantage of host cell machinery to replicate. There are five stages involved in VSV replication that lead to progeny virions. The first stage consists of adsorption of virions, via the $G$ protein, to an unidentified receptor on the host cell membrane and entering the cell through the clathrin-mediated endocytic pathway (139). The second stage, uncoating, involves the 


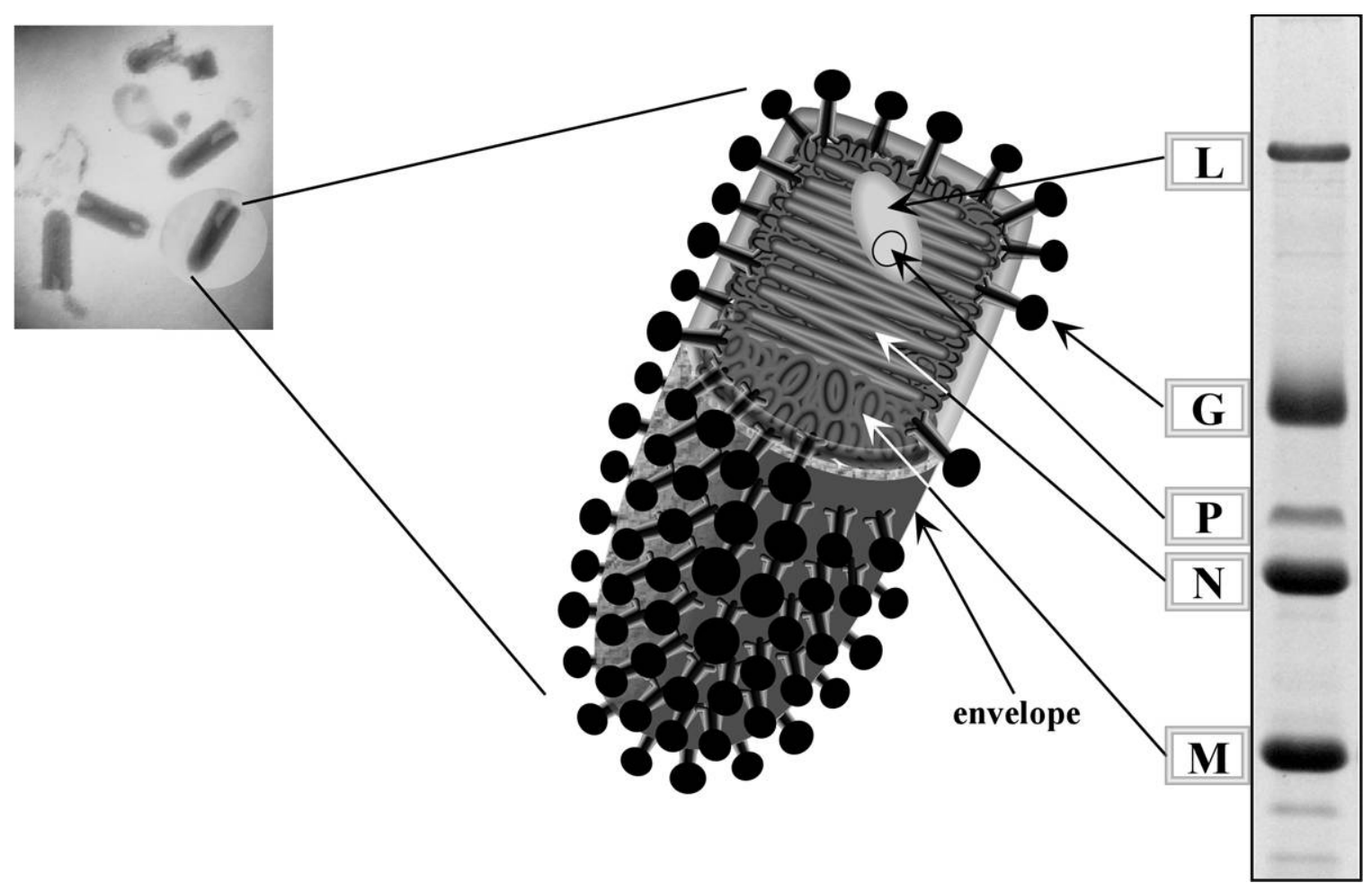

Figure 1-1. VSV schematic.

Electron Micrograph of VSV particles with a cartoon denoting viral proteins (note the skeleton within the viral envelope of the cartoon) and their location within virions and an SDS-PAGE gel. L, large polymerase protein, G, glycoprotein, P, phosphoprotein, N, nucleocapsid protein, M, matrix protein. Illustration from the lab of Michael A. Whitt, Ph.D. with permission from Clint Robison, Ph.D (personal communication). 
$\mathrm{pH}$-dependent fusion of the viral membrane with the endosomal membrane through the activity of $\mathrm{G}$ protein which results in the release of the RNP core into the cytoplasm where replication occurs (139). Either directly after or concomitant with membrane fusion, M protein dissociates from the RNP core resulting in decondensation of the skeleton, which when combined with membrane fusion results in completion of VSV uncoating $(130,174)$. However, the trigger for $M$ protein dissociation within a host cell is unknown at this time. In the third stage, the decondensed, RNP serves as a template for transcription of viral mRNAs by the packaged RNA-dependent RNA polymerase. VSV transcription, like other nonsegmented negative strand RNA viruses, is sequential and polar where each of the five genes is transcribed in the order that they appear from the 3 ' end of the genome $(87,88,130)$. There is a $\sim 30 \%$ reduction in the amount of downstream message that is made when compared to the upstream message, resulting in $\mathrm{N}$ mRNA being the most abundant message and L mRNA the least abundant (203). The viral mRNAs are capped, methylated, and polyadenylated by the VSV polymerase $(85$, 186). Once primary transcription has occurred, stage four begins where the VSV polymerase uses the same encapsidated genomic RNA as a template to synthesize a fulllength, anti-genomic replicative intermediate (RI). The RI functions as a template for replication of more genomic RNA which can be used in subsequent rounds of transcription, replication, and for packaging into progeny virions. Stage five involves the assembly of the VSV virion which can be divided into three phases: (i) encapsidation of newly made genomic RNA by the $\mathrm{N}$ protein and subsequent association of $\mathrm{L}$ and $\mathrm{P}$ forming the RNP, (ii) condensation of RNP by the M protein and association with the plasma membrane forming the skeleton, and (iii) budding from the host cell surface by envelopment of the skeleton and then release of the progeny virion. The last two phases of stage five are attributed to the activity of M protein $(10,67,100,130,132,153,154)$.

\section{The VSV Matrix (M) Protein}

The M protein. M, the smallest of the virion's proteins at 229 amino acids (26 $\mathrm{KDa}$ ), is an unglycoslylated protein that is present at about 1800 copies per virion. It is composed of a pleomorphic $\mathrm{N}$ terminus (residues 1-58) and a globular $\mathrm{C}$ terminus (residues 59-229) made up of 7 beta sheets that "cap" three alpha helices (Fig. 1-2). The M protein plays a key role in virus assembly and budding at the host cell's plasma membrane. It has the ability to bud vesicles from cells when it is expressed in the absence of other VSV proteins (97), a property attributed to its PPxY (PY) motif that binds WW domains of cellular proteins involved in multivesicular body formation (80). Around $10 \%$ of VSV M is associated with membranes in infected cells, whereas the remaining amount is cytoplasmic $(30,31,146,147)$ and nuclear $(133)$. M protein, being multifunctional, is also involved in the cytopathic effects inflicted on host cells infected

by the virus. These effects include inhibition of host mRNA expression (14), translation (181) and nucleocytoplasmic transport (53), and the disruption of host cell cytoskeletal organization (92). Over the past thirty years, many discoveries have been made concerning M protein's role in virus assembly and host cell cytopathic effects. However, key questions still exist, including: what causes the matrix protein to dissociate, where 
A
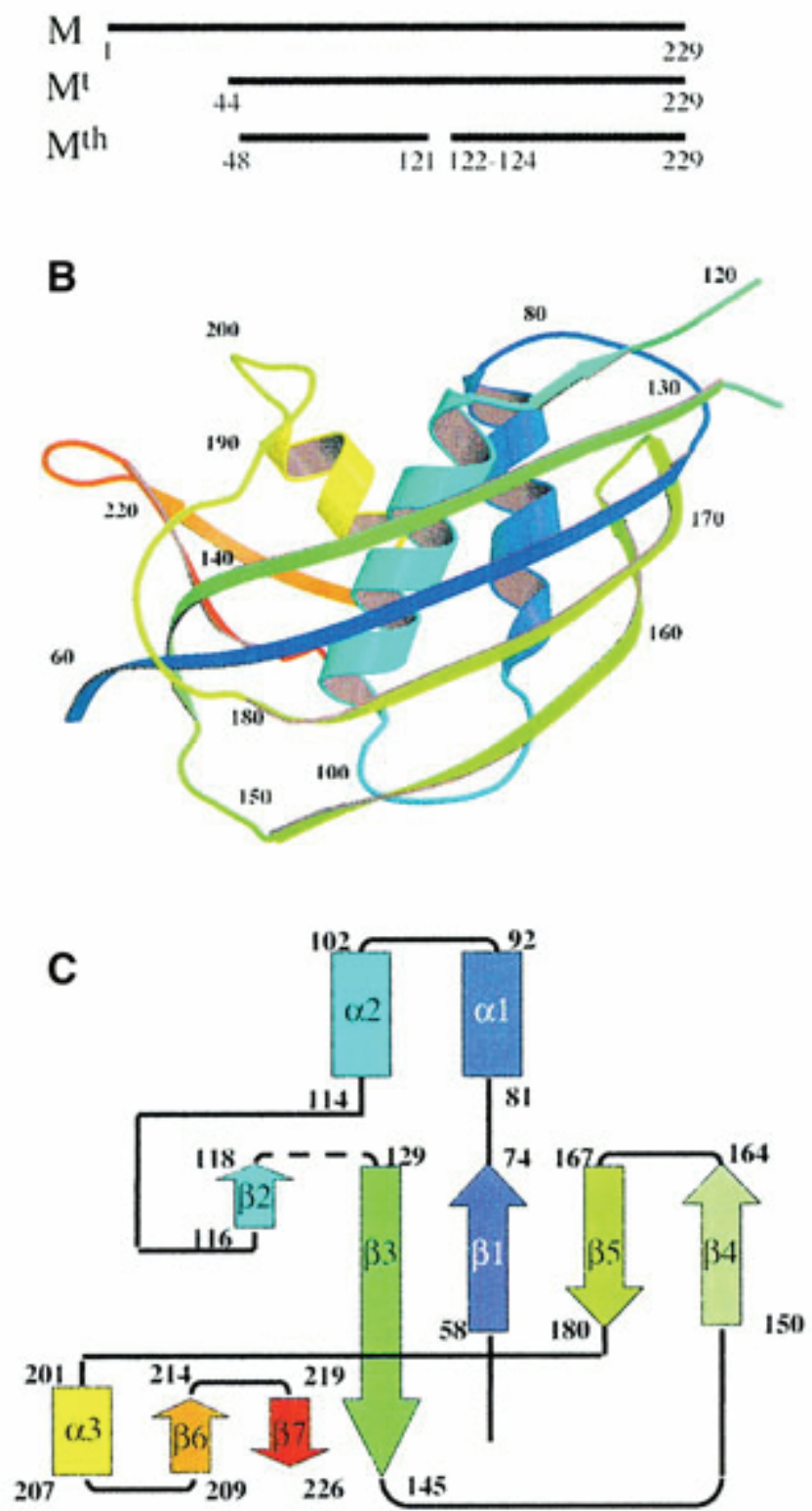

Figure 1-2. Crystal structure of the VSV M protein.

(A) Schematic of full-length $\mathrm{M}$ and the M proteins proteolytically cleaved by trypsin and thermolysin, respectively. (B) Structure of the thermolysin cleaved globular core of the $M$ protein with 7 beta sheets surrounding 3 alpha helices also shown in (C). The exposed loop is located at amino acids 120 to 129 . Reprinted with permission from Marcel Knossow, Gaudier, M., Y. Gaudin, and M. Knossow. 2002. Crystal structure of vesicular stomatitis virus matrix protein. Embo J 21:2886-92, (66). 
does it distribute within cells during viral uncoating events, and which region(s) of $\mathrm{M}$ interact during virion assembly?

$M$ protein and membrane association. A fraction of $M$ protein within infected cells is associated with the inner leaflet of the plasma membrane (31). This localization at the plasma membrane can occur even in the absence of other viral proteins. There are no significant stretches of hydrophobic residues in the $\mathrm{M}$ protein that would serve as a transmembrane region (120) nor are there any modifications by fatty acids to explain its plasma membrane association. The $\mathrm{N}$ terminal region of $\mathrm{M}$ has been implicated in the interaction with membranes via the hydrophobic, photo-reactive probe ${ }^{125}$ I-TID (120). Also the $\mathrm{N}$ terminus of $\mathrm{M}$ contains a highly basic region which might be able to interact with the negatively charged head groups of the membrane phospholipids by electrostatic interactions. Though a logical assumption, deletion of the 43 residues in the $\mathrm{N}$ terminus, which includes the basic region, only weakens membrane association and does not completely reduce the interaction of $\mathrm{M}$ with membranes (30). This observation points to perhaps another membrane interaction domain, which is speculated to be between residues 88-119 (217) and/or a hydrophobic surface located close to a hydrophobic loop that is surrounded by conserved basic residues $(30,66)$. VSV M cell membrane association is necessary for its budding activity (119) and this activity is important for virion release and hence virus assembly (91).

Formation of skeletons by M. Along with $M$ association at the inner leaflet of the plasma membrane, RNP condensation by M is also important in VSV assembly (132) and this occurs at the plasma membrane (157). It has been shown that cytosolic and membrane bound M can bind to RNPs but the proteins have a higher affinity for RNPs isolated from virus versus cytosolic RNPs (57). These observations suggested that $\mathrm{M}$ binding to RNPs is dependent on a change in cytosolic RNPs at the plasma membrane and recent evidence has led to a model where $G$ and RNPs associate in microdomains at budding sites and then $\mathrm{M}$ is incorporated (195). Treating purified VSV virions with detergent at low ionic strength solubilizes the viral membrane and G protein liberating the highly condensed, tightly coiled skeleton which consists of the RNP and M protein $(153,154)$. When these skeletons are placed in the presence of high ionic strength buffer $(\sim 250 \mathrm{mM} \mathrm{NaCl})$ the result is decondensation of these compact structures by dissociation of $\mathrm{M}$ protein. The decondensed RNPs can reform skeletons by dialyzing away the salt in the presence of $\mathrm{M}$ protein. The association of $\mathrm{M}$ and subsequent condensation of RNPs into skeletons results in an inhibition of viral transcription in vitro as well as in infected cells $(26,44,166)$. The $\mathrm{N}$ terminus of $\mathrm{M}$ is essential for binding to the nucleocapsid in vitro $(30,158)$, but proteolytic cleavage of skeletons showed that the $\mathrm{N}$ terminus is exposed to proteases when bound to nucleocapsids suggesting an indirect interaction between this region and nucleocapsids (100).

Membrane associated $M$ protein and its association with the glycoprotein (G) protein. The G protein of VSV is incorporated into virions at budding sites along the 
plasma membrane and this has been shown to occur in microdomains that are not detergent resistant (21) but that are enriched for sphingomyelin and cholesterol $(111,125$, 126). It has been suggested that $G$ is the protein that forms budding sites where the internal viral components can then finish the process of forming a progeny virion (21). G and $\mathrm{M}$ have been shown to interact within virions by chemical crosslinking experiments (46) and within cells using fluorescence photobleaching assays which showed mutations in the $\mathrm{M}$ protein have an effect on the mobility of the G protein on the surface of the cells (173). Purified M protein and skeleton complexes were also shown to interact with and stabilize $G$ protein into trimers in in vitro assays (128). However, the amino acids of the $\mathrm{G}$ protein cytoplasmic tail are not specific to this interaction. In fact the cytoplasmic tail of G just needs to be 1 amino acid in length in order to be incorporated into VSV virions (210). The ability to pseudo-type VSV with foreign glycoproteins also adds to the data that suggests the interaction between G, M, and skeletons is a non-specific interaction (184). Although VSV has a non-specific G cytoplasmic tail interaction in assembly, the $\mathrm{G}$ cytoplasmic tail of rabies virus is important for incorporation into rabies virions (149). This observation suggests the interaction between the cytoplasmic tails of each virion's $G$ protein with their respective skeleton is different between these two viruses found in Rhabdoviridae. Recently, it has been shown that $\mathrm{G}$ and $\mathrm{N}$ may interact and this interaction allows for the RNP to associate with the M protein at the budding sites (195).

\section{VSV Entry and Uncoating}

Entry of enveloped viruses into the host cell. Virions must penetrate the host's cellular membrane to release their genomes and proteins into the cytosol where they gain access to the host's machinery to replicate. In the case of enveloped viruses, this penetration into the host's cytosol requires a membrane fusion event to take place, which is accomplished by a fusion protein on the viral surface. The membrane that is penetrated in this case can either be the plasma membrane or the membrane of an intracellular organelle along an endocytic pathway.

It is known that enveloped viruses like Sendai virus and HIV-I have $\mathrm{pH}-$ independent fusion proteins and can therefore fuse on the surface of the cell $(52,141)$. The $\mathrm{pH}$-independent nature of fusion for these virions and others like them leads one to assume that fusion only occurs at the plasma membrane but this is difficult to prove as these virus particles are continuously taken up by endocytosis as well.

Overall a majority of enveloped virions enter the cell through the endocytic pathway, giving them the ability to move further into the cell. This allows them to bypass potential barriers and a crowded cytosol (135) using the cytoskeletal machinery involved in endocytic vesicle movement (45) in order to penetrate the cell for replication. Virions that enter the cell along the endocytic pathway like VSV and influenza, use the decreasing $\mathrm{pH}$ of endocytic organelles as a trigger for fusion in order to penetrate the cell (207). 
Uncoating of genomes after entry of enveloped viruses. Virions entering a cell have highly condensed genomes protected by proteins. Before these particles can replicate in a host cell, after fusion and entry, an uncoating event must occur. This uncoating event is described as release of the viral genome in a form that is immediately ready for replication (DNA viruses), transcription ((-) RNA viruses), or translation $((+)$ RNA viruses). Typically, uncoating involves the dissociation of the proteins condensing the genome. Once the genome has uncoated replication of the virus can begin and this process is the opposite of what occurs to package the virus for release. Here lies the dilemma for how a virus can accomplish these two distinct mechanisms for the same protein.

Influenza virus is the best example of how a virus can accomplish this through virus-host interactions. As influenza virus is taken into the cell by endocytosis, the virus encounters decreasing $\mathrm{pH}$ the further it is along the pathway. The virus takes advantage of this $\mathrm{pH}$ decrease in another manner that is not linked with the $\mathrm{pH}$-dependent fusion during entry. Within its viral membrane there is an integral membrane protein called M2 that acts as an ion channel that shuttles the protons in the endosome into the virus (110, 169). In turn the acidification within the virion facilitates the dissociation of the M1 protein away from the viral RNPs of influenza virus $(24,222)$ resulting in enhanced uncoating. This is a fascinating, yet simple approach for a virus to achieve the uncoating dilemma by taking advantage of the different environments during the entry pathway.

VSV and the uncoating dilemma. VSV faces the same intriguing question about what is responsible for the dissociation of M protein from RNPs. Unlike influenza virus, VSV lacks an ion channel protein although previous studies have found at low $\mathrm{pH}$ spike proteins of enveloped viruses such as VSV, Sindbis virus, and Semliki Forest virus can make the viral envelope permeable to propidium iodide $(101,114)$. Also, cells expressing these proteins on the cell surface show a $\mathrm{pH}$-dependent permeability (101, 114). These observations coupled with the knowledge of how influenza virus uncoats would lead one to believe that protons may be able to cross the viral envelope and affect $\mathrm{M}$ in a manner similar to M1. However, in vitro studies did not discover a $\mathrm{pH}$ effect on the $\mathrm{M}$ protein associated with skeletons (130). Instead it was suggested that dissociation of $\mathrm{M}$ protein from skeletons occurs spontaneously as a result of the lack of $\mathrm{M}$ protein in the cytosol since it was observed that association and dissociation of $\mathrm{M}$ at physiological ionic strength with RNPs isolated from cells had characteristics of a dynamic reversible equilibrium (130). The very idea that protons may cross the viral envelope is still an intriguing concept considering that there are at least two populations of $\mathrm{M}$ (membrane and RNP bound) within the virus. The skeleton associated $\mathrm{M}$ has been investigated in previous studies but the effect of $\mathrm{pH}$ on $\mathrm{M}$ within the whole virion has not, which leads to an interesting aspect to examine, namely whether VSV may employ a similar strategy as influenza virus. This strategy may be similar for many of the enveloped virions that use the endocytic pathway for entry. 


\section{$M$ in VSV Assembly}

Budding of VSV progeny from host cells. Enveloped, negative-strand RNA viruses have proteins with late domain motifs that are involved in recruiting the cellular vacuolar protein sorting machinery to bud progeny virions from the host cell. Examples include the gag proteins of retroviruses (165), the Ebola virus VP40 protein (12), rabies virus M protein (80), and VSV M protein (80). These proteins contain either PS/TAP or PPxY (PY) motifs that recruit WW containing ubiquitin ligases used in the cellular fission machinery and/or the ESCRT-I complex $(78,103,194,201)$. VSV has both the PPPY and PSAP motifs at the N-terminus of M, where the PY motif has been shown to be important for budding activity $(80,91)$. The PSAP motif has not been shown to have a major late domain role, but mutations in this region led to attenuation of virus both in vitro and in vivo (86).

$M$ protein and nucleocapsid condensation. As discussed previously, the condensation of RNPs by M protein is a reversible process. Despite the fact that the cytoplasm of VSV infected cells contains high concentrations of both intracellular RNPs and soluble M protein, the two do not colocalize with one another $(133,160)$. The plasma membrane is where M and RNPs interact with each other, suggesting that condensation of RNPs does not begin until they associate with the membranes right before budding $(146,157)$. Also, it has been proposed that an additional priming event is needed in order to initiate the assembly process and may involve specific M nucleation sites (67) or modification of the RNPs by viral or host factors $(57,130,195)$.

The exact location of $\mathrm{M}$ within virions is a controversial topic at this time. The general consensus is that M binds to RNPs and condenses them into skeletons (44, 153, 154). Whether $M$ is inside and/or outside the skeleton is still not clearly defined. The traditional view point is that $\mathrm{M}$ acts as a bridge between the nucleocapsid and the viral membrane; putting it on the outside of the RNP $(157,162,220,221)$. Another point of view is that $\mathrm{M}$ protein may form a rod-like scaffold around which the nucleocapsid is wound $(10,11)$. A more detailed analysis of the virion's structure is needed before one or both of these ideas are accepted. It is clear though that there are two populations inside the virion, the membrane and RNP bound forms. M protein has the ability to selfassociate: purified $\mathrm{M}$ protein was found to be a monomer in high ionic conditions $(500 \mathrm{mM} \mathrm{NaCl})$ in the absence of detergent, and in aggregates in low ionic conditions $(<200 \mathrm{mM} \mathrm{NaCl})(147)$. This self-association under low salt conditions was reversible by treatment with Triton X-100 (147), suggesting that a hydrophobic interaction is involved along with electrostatic interactions seen under the different ionic strength conditions. Furthermore, nucleation sites have been implicated in the ability of $M$ to self-associate, where the $\mathrm{N}$ terminus is important for the creation of these nucleation sites and an exposed loop in the $\mathrm{C}$ terminus is important for recruitment into these sites $(65,67)$. Biophysical studies and electron microscopy have shown the aggregates of $\mathrm{M}$ to be rodlike in shape and to have similar striations like those found in skeletons (67). Suggesting 
that the aggregates have similar morphological features when compared to skeletons and that the exposed loop is important for the assembly of VSV.

Self-association of VSV M protein. The protease-sensitive exposed loop, located between amino acids 120-129, of the M protein has been implicated in virion assembly, specifically in regard to M self-association (66). Cleavage of this loop with the protease thermolysin results in $\mathrm{M}$ that cannot self-associate. In fact, $\mathrm{M}$ self-association was an obstacle in the crystallization of the protein. It was found that cleavage by thermolysin prevented self-association and allowed diffractable crystals to form (66). This cleavage site was different from trypsin sites (loss of pleiomorphic N-terminus) on $\mathrm{M}$ and was found to be between amino acids 120-PAVLADQGQP-129 (65). Full-length $M(M), M$ cleaved by trypsin $\left(M_{t}\right)$, and $M$ cleaved by thermolysin $\left(M_{t h}\right)$ were studied and found to have a decrease in the ability to self-associate in the order $M>M_{t}>M_{\text {th }}$ (65). This exposed loop is also believed to be involved in association of matrix with the plasma membrane, which is where the matrix protein condenses the RNP into a skeleton (66, 157). The crystal structure of the $\mathrm{M}_{\text {th }}$ globular $\mathrm{C}$-terminus revealed that there are seven $\beta$ sheets that cover three core $\alpha$ helices like a "turtle shell" (66). The loop is located on the exterior (thermolysin cleavage) between a large and small $\beta$ sheet that leads to one of the core $\alpha$ helices (66). Mutations in the loop at amino acids 121 to 124 have been studied and were found to have a minor effect on VSV assembly, and also infection of cells with this virus resulted in reduced viral protein synthesis at 6 to 8 hours post infection (35).

\section{VSV Cytopathogenicity and Methionine 51 of M}

Leader RNA transcripts. VSV infection causes a range of cytopathic effects from the shut down of host protein synthesis to the rounding of the cell at later times post infection (138). Two products involved in inhibition of protein and RNA synthesis were implicated in ultraviolet (UV) mapping experiments of which one was the leader RNA transcript $(48,71,206)$. The leader transcript is 45 nucleotides long and is transcribed from the 3' end of VSV's genome before viral mRNAs are made. The role of the leader RNA transcript in RNA synthesis inhibition has been implicated by observations that revealed leader transcripts could be found in the nucleus of a host cell shortly after infection (108), that leader RNA could associate with La protein, a putative RNA polymerase III transcription factor (175), and that leader RNA could inhibit transcription of RNA by the RNA polymerase II and III enzymes in HeLa cell extracts $(72,73,148)$. It must also be said that leader RNA on its own was shown to be insufficient to inhibit host transcription, where a host factor or viral protein(s) may be required for the inhibition $(47,170)$.

Inhibition of host gene expression by $\mathbf{M}$ protein. The viral protein required to inhibit host transcription was found to be $\mathrm{M}$ by in vitro and in vivo studies. Within infected cells the M protein is found in the nucleus (133), and the expression of the 
protein alone in the absence of other viral components results in the inhibition of host directed gene expression (14). In fact, it is difficult to express $M$ from vectors that rely on the host cell transcription machinery $(16,122)$. Inhibition of transcription by $\mathrm{M}$ was shown to be very effective, where it reduced the amount of gene reporter expression by $50 \%$ with 1,000 fold less protein present than the amount found in host cells between 4 and 6 hours post infection (131). M protein has been shown to reduce expression from the IFN $\beta$ promoter (55), which was correlated with inhibition of RNA transcription and protein translation (4). The inhibition of transcription has been linked to interference with RNA polymerase II in a non-promoter-dependent manner and RNA polymerase III in a promoter-dependent manner (3). It has also been shown that $\mathrm{M}$ interferes with transcription factor (TF) IID but most likely in an indirect manner as M could not interfere with TFIID in an RNA polymerase II in vitro transcription assay $(218,219)$.

The inhibition of host gene expression was found to be genetically separable from the assembly functions of M. In a study of the temperature-sensitive (ts) mutant tsO82, where $\mathrm{M}$ has a M51R mutation and is defective in its ability to inhibit host gene expression, it was shown that ts 082 could rescue a group III M mutant tsO23 virus that is defective in assembly (15). This separation of host gene expression inhibition and assembly activity was also shown by observing the MN1 deletion mutant, lacking amino acids 4-21 at the N-terminus, which was assembly defective but was still able to function in inhibition of gene expression (15).

\section{Inhibition of nucleocytoplasmic transport by M protein: implications for} methionine 51. The M protein has multiple cytopathic effects on the host cell and in addition to the inhibition of transcription it can inhibit nucleocytoplasmic transport of host mRNA when the protein is expressed in cells (168). In Xenopus laevis oocytes, M inhibited the import of snRNPs and karyophillic proteins as well as export of snRNAs (60) and mRNAs but not tRNAs (168). This block of transport was shown to inhibit snRNA processing and assembly within 1 hour of infection and may be the first cytopathic effect by VSV (60).

The inhibition of nucleocytoplasmic transport by the $\mathrm{M}$ protein has been shown to be through the interaction with RaeI and the nucleoporin Nup98 at the nuclear pore complex (NPC) $(53,204)$. The region on the M protein that is responsible for this interaction has been mapped to amino acids 51-59 with methionine 51 (M51) being the most important for colocalization to NPCs and inhibition of nucleocytoplasmic transport (168). Recently, it was also shown that the VSV New Jersey M48 and M51 residues are important for inhibiting gene expression, suggesting that these may be involved in a similar mechanism for this M protein (104).

The M51 residue and its role in nucleocytoplasmic transport inhibition has been put to the forefront of oncolytic therapy with $\operatorname{VSV}(50,109,127,193)$. It was shown that attenuated VSVs with a deletion or mutations at the M51 residue were able to exploit the inability of oncogenic cells to respond to interferon, leading to infection and oncolysis. While the cells cannot respond to interferon, they can make interferon and this leads to 
protection of cells that can respond to interferon in vivo $(192,193)$. This is the basis for oncolytic therapy at the present time and will be a part of the future of VSV as an oncolytic vector $(8,9,36,49,62,127,156,171,188,213,214)$.

Cell rounding: as a hallmark of VSV infection. A characteristic of wild-type VSV infections is the loss of a cell's normal morphology resulting in cells that become round $(155,217)$. This ability to round the cells has been attributed to the M protein (16) and the sequential disassembly of the cytoskeleton (190). It was also shown that $\mathrm{M}$ protein could co-immunoprecipitate with tubulin from infected cell extracts and that $\mathrm{M}$ could interact with polymerized and disassembled tubulin in vitro (150). In these studies it was suggested that the highly basic N-terminus was important for this effect as proteolytically cleaved $\mathrm{M}$ did not associate with tubulin as well in vitro and this interaction was indicated through the acidic C-terminus of tubulin (150). This suggestion does not correlate with data from cells expressing the deletion mutant MN1, which is missing amino acids 4-21 and therefore the highly basic region, which lost their morphology and became round (129).

The tsO82 M51R mutation in M protein on the other hand affected the ability to cause the dissociation of the cytoskeleton in cells expressing that protein (129). This observation correlates with data that suggested two additional translation products from the M gene, called M2 and M3, were important for the cell rounding phenotype of the M protein (92). It was found that these products were the result of downstream methionines in the same open reading frame as full-length $\mathrm{M}$, at methionines 33 and 51. When these residues were mutated to alanines (M-33,51A) the translation of M2 and M3 was abolished and cell rounding was delayed without affecting virus assembly. Transient expression of M2 and M3 was sufficient to cause cell rounding and expression of these proteins from a separate cistron in a virus that expressed M-33,51A restored cell rounding, suggesting these two translation products are required for cell rounding (92). These data taken together with the M51R M mutant data suggest that not only is the methionine 51 residue important for inhibition of nucleocytoplasmic transport but also as a start site for a polypeptide of $M$ that contains amino acids 51-229. One could imagine a scenario where this offers VSV an advantage of having different populations of M: (1) a population with the N-terminal amino acids important for membrane binding and budding activity that is used for assembly of progeny virions and (2) a population without those amino acids that cannot be sequestered to the cellular membranes as readily and therefore could interact with NPCs and cause other cytopathic effects. Not only has this been shown for VSV Indiana M protein but it has recently been shown that methionines 48 and 51 of the VSV New Jersey M protein have similar requirements for CPE (104).

M protein and apoptosis. It was found that VSV infection in HeLa cells resulted in fragmentation of chromosomal DNA into nucleosomal oligomers and in nuclear fragmentation, which are cell effects usually associated with apoptosis (107). In fact, it was shown that expression of the M protein from mRNA within HeLa and BHK cells could induce apoptosis because the cells underwent morphological changes and caspase- 3 
was activated (106). It was also suggested that the cell rounding ability of $\mathrm{M}$ was a result of apoptotic activity (105). Comparison of wild-type M and the M51R mutant revealed that these proteins could induce apoptosis differently where wild-type used the mitochondrial induced pathway and was shown to be sensitive to over expression of Bcl2 (63). In contrast, the M51R mutant could induce apoptosis through the death receptor pathway and required activation of caspase-8 (63) through PKR, Fas, and Daxx signaling (62).

\section{Enveloped RNA Viruses: Origins and M Proteins}

Origin and evolution of RNA viruses. The origin of "the ancestor" nsRNA virus and the relationship between this ancestral virus and present day virions that are continually evolving is a topic that is not easily addressed. Unlike the evolutionary study of animals and plants, there is no fossil record of their existence over billions of years. All that can be studied are the genomes and their products, the replication strategies, and host ranges of viruses to make any inference about where they may have originated in terms of evolution. Based on these observations viruses have been classified by either the International Committee on Taxonomy of Viruses (ICTV) or by the Baltimore classification system $(7,140)$. Evolution is a consequence of the reproductive success of individuals and their phenotypes that have an influence on the subsequent genotypes that occur from generation to generation. Due to their self-replication and polymerase fidelity mutations can occur that increase the fitness of nsRNA viruses. The escape from host antiviral responses by HIV-I (37) and changes in host range by influenza A (205) are a few of the examples of the evolution of some RNA viruses whether they be short or long term changes.

M proteins of enveloped RNA viruses. Enveloped RNA viruses use M proteins to condense RNPs and to link RNPs to the virus membrane. In the case of retroviruses, which have a precursor gag polyprotein consisting of the matrix (MA), capsid (CA), and nucleocapsid (NC) proteins, the CA protein condenses the genome and the MA is responsible for RNP association with the virus membrane. Though similar in function, the sequence homology of the $\mathrm{M}$ proteins from filo-, rhabdo-, myxo-, and paramyxovirus and retroviral MA do not show clear homology although the L domains of PS/TAP or PY motifs are similar in these proteins that function in budding of virions from host cells (40, $79,80,119,216)$. Comparison of the structural homology has been examined by structural biologists in reviews of the literature between Ebola virus VP40, VSV M, and HIV MA and these were found to have no structural homology (200), but a comparison between influenza virus M1 and HIV MA and CA proteins revealed that the M1 protein may have a combination of the important structural assembly elements of the MA and CA proteins (77). Taken together these observations show that trying to find the evolutionary link between proteins from RNA viruses is a complicated endeavor.

Proteins that have the same function can just be a result of necessary functions needed to release protected genomes from cells so the virus has acquired (or evolved) a protein for 
this function. Alternatively, these proteins may have structures that have arisen for the functions suggesting modular evolution.

\section{Hypotheses}

Hypothesis 1. Based on the literature discussed in this introduction, the first hypothesis investigated in this dissertation is that VSV uses host cell factors/processes for dissociation and distribution of the matrix protein during uncoating (Chapters 2 and 3 ). The areas of investigation for this hypothesis are indicated by (1) and (2) respectively, in Figure 1-3.

Hypothesis 2. The second hypothesis investigated is that the amino acid sequence of the exposed protease-sensitive loop of the M protein is important for VSV assembly (Chapter 4) at the site indicated by (3) in Figure 1-3. 


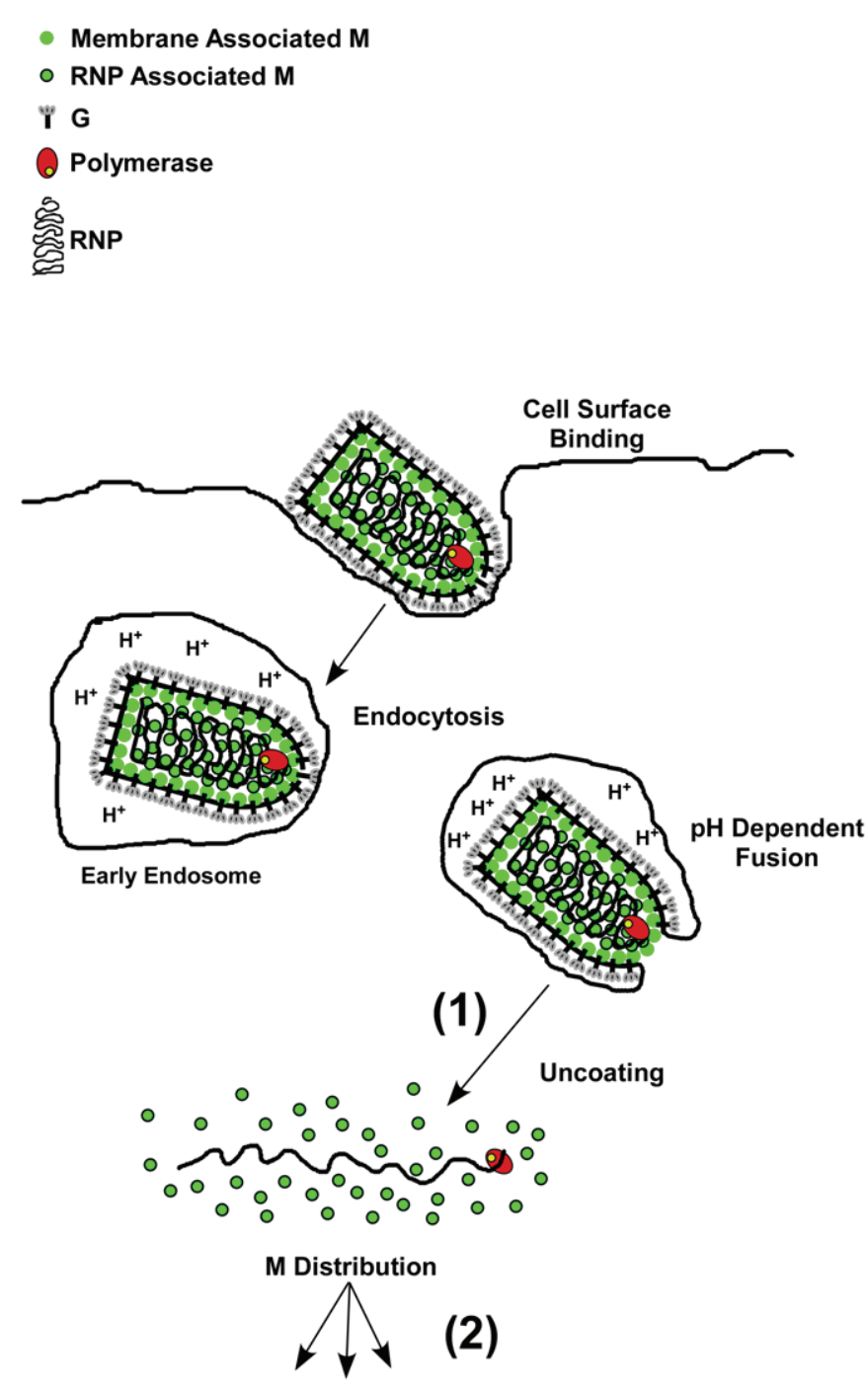

VSV Entry

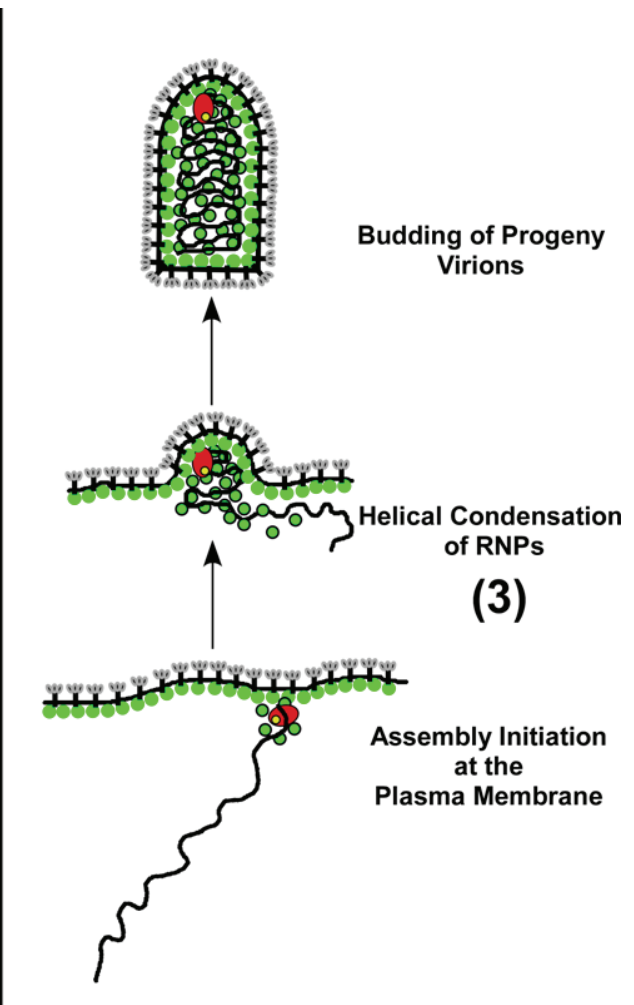

VSV Assembly

\section{Figure 1-3. Hypotheses model.}

(1) Location within the VSV entry pathway where the question of what cellular factor is involved in uncoating will be addressed. (2) After dissociation of M from RNPs what cellular processes are involved in M distribution? (3) Location in assembly of VSV where the question of the amino acids in the protease-sensitive loop will be examined. 


\section{Chapter 2: Analysis of Matrix Protein Distribution after Vesicular Stomatitis Virus Uncoating}

\section{Introduction}

Entry of enveloped viruses through the clathrin-mediated endocytosis pathway generally occurs by attachment to the cell surface and uptake of virions in coated vesicles that are transported through the endosomal pathway to early or late endosomes. At early or late endosomes there is a $\mathrm{pH}$ induced fusion of the endosomal and viral membrane, which results in virus uncoating and release of the genome $(114,136)$. Vesicular stomatitis virus (VSV), a prototype enveloped, nonsegmented, negative-strand RNA virus in the family Rhabdoviridae, enters the host cell through this $\mathrm{pH}$-dependent endocytic pathway (139). VSV's genome encodes five major viral proteins: the nucleocapsid protein $(\mathrm{N})$, the phosphoprotein $(\mathrm{P})$, the matrix protein $(\mathrm{M})$, the glycoprotein $(\mathrm{G})$, and the large polymerase protein $(\mathrm{L})$. The viral genome is encapsidated by the $\mathrm{N}$ protein and associates with the viral RNA-dependent RNA polymerase (complexes of $\mathrm{L}$ and $\mathrm{P}$ proteins), which together form the ribonucleoprotein (RNP) complex. The M protein within virions is associated with RNPs in structures called skeletons $(153,154)$ and a separate membrane associated population of $\mathrm{M}$ anchors the skeletons to the viral membrane $(31,151)$, which has transmembrane $G$ protein spikes that protrude from the membrane. The $\mathrm{G}$ protein is responsible for attachment of virions and fusion of the endosomal and viral membranes $(18,82,134,161,198,211)$ and results in the transfer of the genome into the cytoplasm where VSV replication occurs following fusion (139). Either directly after or concomitant with membrane fusion, $\mathrm{M}$ protein dissociates from the RNP core which results in the decondensation of the skeleton $(153,154)$ and completes virus uncoating (174). After uncoating, the decondensed RNP serves as a template for transcription of viral mRNAs by the packaged RNA-dependent RNA polymerase.

Virus uncoating, defined as the dissociation of M from RNPs is an essential step which is required for a productive infection (130); without this occurrence it is thought that transcription of viral mRNAs will not proceed since it has been shown that transcription from RNPs is inhibited by M protein $(32,44,212)$. This essential step is also shared by another enveloped negative-strand virus, influenza, which requires removal of its matrix protein (M1) for a productive infection $(102,137)$. To better understand this essential uncoating step, we wanted to develop a recombinant VSV (rVSV) that would allow us to visualize the release of M protein in live cells.

Five different rVSVs encoding fluorescent $\mathrm{M}$ proteins were constructed for this study. Three of these contained M fused to green fluorescent protein (GFP). This strategy has been used to generate rVSVs that encoded fluorescent G (G-GFP) and P (PeGFP) proteins which were used to examine virus entry and assembly $(41,42)$. In previous studies, GFP has been fused to $\mathrm{M}$ at the $\mathrm{N}$ and $\mathrm{C}$ termini to observe $\mathrm{M}$ protein expression, function, and localization in the absence of other viral proteins $(69,123,168)$, but these chimeric proteins were not studied in the context of virus assembly. Here we 
fused GFP to the C-terminus of M (MFC) to avoid the important regions on the Nterminus involved in membrane association $(30,31,120,217)$ and virus budding $(80,91)$. Unexpectedly, the M-GFP chimeras had reduced budding activity when compared to M wild-type (Mwt), and rVSVs that encoded MFCs as the sole M protein were unable to be recovered; however virus encoding Mwt and MFC as an additional gene between $\mathrm{G}$ and L (rVSV-M-MFC) was recovered, but the amount of MFC incorporated into virions was low and we were unable to detect the virus bound to cells by fluorescence microscopy.

In an alternative approach we fused a tetracysteine (-CCRECC-) Lumio $^{\mathrm{TM}}$ tag to $\mathrm{M}$ and recovered infectious virus. Lumio $^{\mathrm{TM}}$ technology has been used previously to study Ebola VP40 membrane localization (164) and to label human immunodeficiency virus-1 (HIV-1) integrase for live cell HIV-1 entry (5). Using in vivo labeling with Lumio $^{\mathrm{TM}}$ Green and purification of labeled virus we visualized VSV entry and the subsequent fate of M-Lumio-Green in fixed and live cells.

We show that after fusion of rVSV-M-Lumio-Green with endosomes, the bulk of M-Lumio-Green remains associated with recycling endosomes but some also localizes to the nuclear envelope. The delivery of M-Lumio-Green to the nuclear envelope was not dependent on actin or tubulin suggesting this may occur by diffusion. Using confocal microscopy we observed that RNPs physically separated from M-Lumio-Green on the endosomal membrane and that the release of RNPs into the cytoplasm was also not dependent on microtubules. We believe the release of RNPs seen after rVSV-M-LumioGreen virions have fused with endosomes is a measure of an uncoating event and therefore defines the start of a productive infection for rVSV virions.

\section{Materials and Methods}

Plasmid design and construction. Construction of the cDNAs of MFC-II, -III, and -IV (MFC) illustrated in Figure 2-1A was accomplished using pBluescript(BS) MФT (wild-type; Indiana) as a vector and overlapping PCR to fuse the N-terminus of GFP to the C-terminus of M. Primers (GFP-M-C-Link-C, CEM 4, or 5) which had a 5' NheI site followed by sequences encoding an 8 (-II), 15 (-III), or 20 (-IV) amino acid (Gly-Ser) linker that joined to the initiating methionine of GFP, were used together with a noncoding primer (GFP-NdeI-NC) to amplify a PCR fragment that served as a link between $\mathrm{M}$ and GFP in a triple ligation of gel purified fragments. The M-GST cDNA was constructed by fusing the $\mathrm{N}$-terminus of GST to the $\mathrm{C}$-terminus of M using an 8 amino acid $\left(\mathrm{Gly}_{3}-\mathrm{Ser}\right)_{2}$ linker in a similar way but using the primer 5'GST-cod to join the linker to the initiating methionine of glutathione-S-transferase.

pBS-M-Lumio was constructed by annealing two overlapping oligonucleotides that encoded the tetracysteine $\left(^{*}\right)$ Lumio $^{\mathrm{TM}}$ tag (Fig. 2-1B, amino acids 230-246) to the Cterminus of M. A coding and non-coding oligonucucleotide (CEM6 and CEM7) that encoded the amino acids in the tag were phosphorylated with $\mathrm{T} 4$ polynucleotide kinase 
A

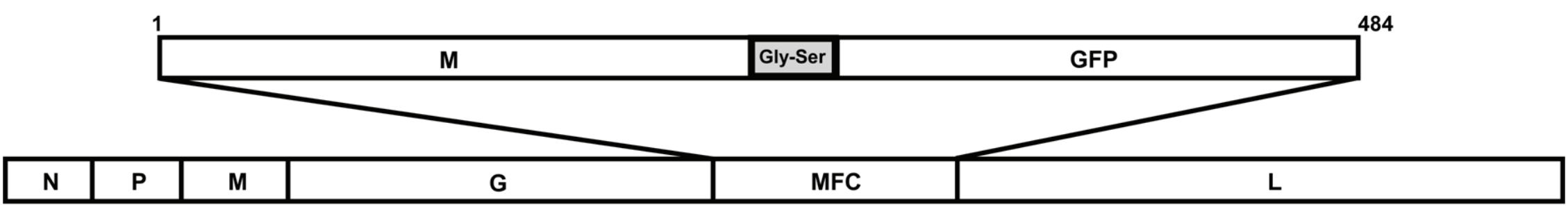

rVSV-M-MFC

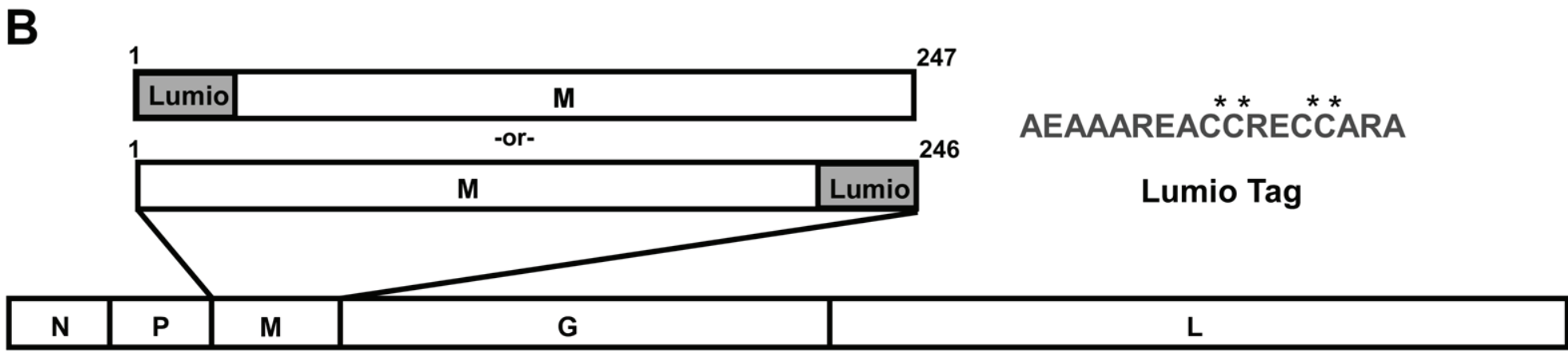

rVSV-M-Lumio

Figure 2-1. Schematic of fluorescent $M$ proteins and rVSV genomes.

(A) Depiction of the MFC protein and the organization of the rVSV-M-MFC genome. (B) Illustration of the Lumio-M and M-Lumio proteins. Amino acid positions are designated by numbers and the amino acid sequence of the Lumio $^{\mathrm{TM}}$ tag (gray box) at the C-

terminus is shown. The Lumio-M protein contains an initiating methionine on the N-terminus of the Lumio-tag. The cysteines that bind the biarsenic Lumio ${ }^{\mathrm{TM}}$ reagent are denoted by ${ }^{*}$ symbols. The location of Lumio-M or M-Lumio in the viral genome is shown. 
and were annealed to form a linker with NheI and EagI overhangs, and used to ligate into the vector pBS-M-Flag-C digested with NheI and EagI.

The pBS-Lumio-M plasmid was constructed by using PCR amplification of pBSMФT as a template with a primer containing KpnI and $A s c$ I restriction sites, a Kozak sequence (TTCATCATGG), and the sequence for the Lumio ${ }^{\mathrm{TM}}$ tag at the 5 ' end which overlapped with the first 24 bp of the coding sequence of $\mathrm{M}$ (CEM8) and a 3' reverse T3 primer. The product was digested with KpnI and EagI, gel purified, and ligated into pBSMФT digested with the same enzymes. Sequences of constructed cDNAs were determined by the Molecular Resources Center at the University of Tennessee Health Sciences Center and no changes from predicted sequences were found.

Generation of full-length VSV cDNAs encoding MFC, M-GST, M-Lumio, and Lumio-M. The MFC, M-GST, M-Lumio, and Lumio-M chimera sequences were cloned into the polylinker (PL) of a modified version of the VSV anti-genome $\mathrm{p} \Delta \mathrm{M}-\mathrm{PLF}$ (91) except there was no GFP between the $\mathrm{G}$ and L coding regions. The resulting plasmids were called pVSV-MFC-II, -III, -IV, pVSV-M-GST, pVSV-Lumio-M, and pVSV-Lumio-M. For rVSV plasmids encoding Mwt and the MFC chimeras together, the cDNAs for MFC-II, -III, and -IV were cloned into the polylinker of pVSV-GL-PL (92) located between the $\mathrm{G}$ and $\mathrm{L}$ genes.

Recovery and characterization of recombinant VSV (rVSV). Recombinant viruses were recovered using reverse genetics (116) with some modifications as described previously (91). Single-step growth curves were performed by adsorbing virus to baby hamster kidney (BHK-21) cells in $35 \mathrm{~mm}$ plates at a multiplicity of infection (MOI) of 10 for 1 hour at $31^{\circ} \mathrm{C}$ while continually rocking. The inoculum was removed, the cells were washed 4x with serum-free Dulbecco's minimal Eagle's medium (DMEM) to remove unbound virus, DMEM containing 5\% fetal bovine serum (FBS) was added, and the cells were placed at $37^{\circ} \mathrm{C}$. Every two hours post infection (hpi) a $5 \%$ aliquot of media was collected, replaced with the same volume of fresh media, and then virus titers were determined in duplicate by plaque assay on BHK-21 cells. Growth curves were performed in triplicate for each virus.

Transient expression of MFC, M-Lumio, and Lumio-M proteins. BHK-21 cells at $\sim 90 \%$ confluency on glass cover slips in $35 \mathrm{~mm}$ dishes, were infected with a modified vaccinia virus (vTF7-3) encoding T7 RNA polymerase (61) at MOI 5 for 1 hour at $31^{\circ} \mathrm{C}$ in SF-DMEM. The inoculum was removed and the cells were transfected with $5 \mu \mathrm{g}$ of pBS-MФT (wild-type; Indiana), pBS-MFC-II, -III, or -IV, pBS-M-Lumio, or pBS-Lumio-M using $20 \mu 1$ TransfectACE in SF-DMEM (180). Five hours post transfection (p.t.) the transfection mix was removed and replaced with 5\% FBS DMEM containing antibiotics (100U/ml streptomycin and penicillin), and 18 hours p.t. the cells were fixed and processed for immunofluorescence or were used in a budding assay as described below. 
Immunofluorescence (IF) staining and confocal microscopy. Cells either transiently expressing $\mathrm{M}$ proteins from plasmids or infected with rVSV were washed twice with phosphate-buffered saline (PBS) and then fixed for 15 minutes with 3\% paraformaldehyde (PFA) in PBS at room temperature (r.t.). The fix solution was then removed and the cells washed twice with PBS containing $10 \mathrm{mM}$ Glycine and $0.05 \%$ sodium azide (PBS-Glycine), and then permeabilized with 1\% Triton X-100 in PBSglycine at r.t. for 1 minute. After permeabilization, the cells were washed twice with PBS-Glycine and then stained for; $\mathrm{M}$ protein using an anti-M monoclonal antibody ( $\mathrm{mAb}$ 23H12) conjugated to rhodamine, VSV N protein using anti-N mAb 10G4 conjugated to rhodamine, Nup62 using anti-Nup62 mAb (\# 610497, BD Biosciences), mannose 6 phosphate receptor (M-6-P-R) using anti-M-6-P-R mAb (\# MA1-066, Affinity BioReagents), LAMP-1 using anti-LAMP-1 mAb (Developmental Studies Hybridoma Bank at the University of Iowa), or microtubules using anti- $\alpha$ tubulin mAb (\# A-11126, Molecular Probes). The unconjugated primary antibodies were detected with a goat antimouse secondary antibody conjugated to rhodamine (Jackson Research Laboratories) or for anti-Nup62 we used Zenon Goat anti-mouse IgG2b Alexa Fluor 647 (\# A-21242 Invitrogen; Molecular Probes). Phalloidin conjugated to Texas Red-X (Invitrogen; Molecular Probes) was used to stain actin. The distribution of the indicated proteins was examined using laser scanning confocal microscopy (Zeiss LSM 510 AIM version 3.2 software). Optical slices of $1 \mu \mathrm{m}$ were captured using 488nm, 543nm, and 633nm laser excitation. Quantification for colocalization of $\mathrm{M}$ and $\mathrm{N}$ protein was accomplished using the "Profile" function of the Zeiss LSM software by counting the number of N protein foci that colocalized with $\mathrm{M}$ in a total of 50 cells from three different experiments.

Budding assays. Cells transiently expressing the indicated $M$ protein were washed twice with methionine-free, SF-DMEM and then incubated in this media for 15 minutes to deplete the intracellular methionine pools at 5 hours p.t. After depletion, a media composed of 1 part 5\% FBS DMEM to 9 parts methionine-free media and supplemented with $50 \mu \mathrm{Ci}$ of ${ }^{35} \mathrm{~S}$-Methionine (Met) Express Protein Labeling Mix (Perkin-Elmer) per ml was added and 18 hours later media and cells were collected separately and processed for immunoprecipitation (IP).

The media were centrifuged at $170 \mathrm{x}$ g for 10 minutes to remove dislodged cells and the supernatant was placed on ice after addition of 200U of aprotonin (U.S. Biochemicals). The supernatant was made to $50 \mathrm{mM}$ Tris- $\mathrm{HCl}, 150 \mathrm{mM} \mathrm{NaCl}, 0.1 \% \mathrm{NP}-$ 40, $1 \mathrm{mM}$ EDTA, $20 \mathrm{mM} \mathrm{NaN}_{3}, 0.3 \% \mathrm{SDS}$, and radio-labeled $\mathrm{M}$ proteins were immunoprecipitated with $\mathrm{M} \mathrm{mAb}(23 \mathrm{H} 12)$ overnight at $4^{\circ} \mathrm{C}$ and retrieved using Pansorbin (\#507861, CALBIOCHEM).

Cell extracts were prepared by washing the cells twice with PBS and then lysing with $800 \mu \mathrm{l}$ of detergent solution (10 mM Tris-HCl [pH 7.4], $66 \mathrm{mM}$ EDTA, 0.4\% sodium deoxycholate, $1 \%$ Triton $\mathrm{X}-100,0.05 \% \mathrm{NaN}_{3}$ ) containing $200 \mathrm{U} / \mathrm{ml}$ of aprotonin for 5 minutes at r.t. on a rocker. The cell extract was collected, nuclei and insoluble material removed by centrifugation at $10,000 \times \mathrm{g}$ for 5 minutes and a $200 \mu 1$ aliquot of the cell extract was made to $0.3 \%$ SDS and immunoprecipitated with M mAb (23H12) 
overnight at $4{ }^{\circ} \mathrm{C}$ followed by Pansorbin retrieval. One-quarter of the radio-labeled $\mathrm{M}$ proteins in the cell extract were compared to the amount released into the supernatant by SDS polyacrylamide gel electrophoresis (PAGE) on a 9\% polyacrylamide gel and visualized by fluorography.

Immunoblot analysis of rVSV-wt and rVSV-M-MFC virions. To detect the presence and location of M-wt and MFC within virions, $1 \mu \mathrm{g}$ of protein $\left(\sim 1 \times 10^{7} \mathrm{PFU}\right)$ from purified rVSV-wt or rVSV-M-MFC was solubilized by $0.1 \%$ Triton X-100 TN Buffer (10 mM Tris- $\mathrm{HCl}$ [pH 8.0], $150 \mathrm{mM} \mathrm{NaCl})$ in a total volume of $1 \mathrm{ml}$ or left untreated. One $\mathrm{ml}$ of each solubilized virus preparation was then ultracentrifuged over 3.5 $\mathrm{ml}$ of a $20 \%$ sucrose cushion $(10 \mathrm{mM}$ Tris- $\mathrm{HCl}$ [pH 8.0], $150 \mathrm{mM} \mathrm{NaCl})$ at 45,000 rpm for 35 minutes in an AH-650 swinging bucket rotor (Sorvall). After centrifugation $1 \mathrm{ml}$ of the supernatant was collected and the proteins in this fraction were precipitated by adding trichloroacetic acid (TCA) to a final concentration of 10\%. Viral proteins in the pellet (RNP fraction) and the supernatant were separated by SDS-PAGE on a 10\% polyacrylamide gel and then transferred to a polyvinylidene fluoride (PVDF) microporous membrane (Immobilon-P; Millipore). Monoclonal antibodies to M (23H12) and GFP (\# MMS-118R Covance) were used to detect each protein followed by addition of a goat anti-mouse antibody tagged with horseradish peroxidase (Jackson Research Laboratories). Proteins were located using a chemiluminescence kit (Immobilon Western; Millipore) according to the manufacturer's instructions.

Lumio $^{\text {TM }}$ Green labeling and quantification of rVSV. BHK-21 cells on 100 mm plates at $\sim 95 \%$ confluency were infected with rVSV-wt, rVSV-M-Lumio, or $\Delta \mathrm{G}-\mathrm{M}$ Lumio at MOI 1. The inoculum was removed after 1 hour, the cells were washed two times with SF-DMEM and $10 \mathrm{ml}$ of 5\% FBS DMEM was added. At 4 hpi the cells were washed twice with reduced-serum Opti-MEM I media (GIBCO) and replaced with $10 \mathrm{ml}$ of Opti-MEM I containing $200 \mathrm{nM}$ Lumio $^{\mathrm{TM}}$ Green (Invitrogen). Eighteen hpi the supernatant was collected and labeled virus was concentrated by centrifugation over a $20 \%$ sucrose cushion at 38,000 rpm in an SW41 swinging bucket rotor (Beckman) for 45 minutes. The viral pellet was resuspended on ice in $1 \mathrm{ml}$ of $10 \%$ sucrose $\mathrm{TN}$ buffer. Residual, unbound Lumio ${ }^{\mathrm{TM}}$ Green reagent was removed from the resuspended virus preparation by adding $100 \mu \mathrm{l}$ aliquots to Sephadex G-50 (Fine) columns (Roche). The void volume containing the virus was collected and stored at $-80^{\circ} \mathrm{C}$ except for $\Delta \mathrm{G}$ virions which were used for experiments on the same day of preparation. Titers were determined by standard plaque assay on BHK-21 cells and protein concentration was determined by a BCA protein assay (Pierce) according to the manufacturer's directions.

The amount of fluorescence in the virus suspensions was determined by adding one microgram of viral protein to a cuvette that contained $3 \mathrm{ml}$ of HMTN buffer $(10 \mathrm{mM}$ HEPES, $10 \mathrm{mM}$ MES, $10 \mathrm{mM}$ Tris-HCl [pH 8.0], $150 \mathrm{mM} \mathrm{NaCl}$ ) using a Perkin-Elmer Luminescence Spectrometer (LS 50B). Protein-specific fluorescence was examined by dissociating virus in SDS-PAGE sample buffer containing one-tenth the normal concentration of $\beta$-mercaptoethanol. Viral proteins were resolved on an SDS-10\% 
polyacrylamide gel and the gel was analyzed for fluorescent protein bands using a STORM 860 fluorescence imager $(480 \mathrm{~nm})$ and Imagequant software (Molecular Dynamics). The same gel that was scanned for fluorescence was processed for immunoblot analysis using the anti-M antibody (23H12) and proteins were visualized as described above.

Live-cell synchronized entry assay. BHK-21 cells plated onto $35 \mathrm{~mm}$ glass bottomed culture dishes (MatTek Corporation) at $90 \%$ confluency were washed twice with $2 \mathrm{ml}$ of ice-cold Opti-MEM I and then placed at $4^{\circ} \mathrm{C}$ for 10 minutes. Lumio-labeled virus (MOI 50) and human transferrin conjugated to Texas Red (50 $\mu \mathrm{g})$ were adsorbed for 90 minutes in $100 \mu \mathrm{l}$ ice-cold Opti-MEM I with rocking every 15 minutes at $4^{\circ} \mathrm{C}$. To initiate entry, the ice-cold inoculum was replaced with Opti-MEM pre-warmed to $37^{\circ} \mathrm{C}$ and the cells were observed by laser scanning confocal microscopy on a heated stage set to $37^{\circ} \mathrm{C}$ and equipped with an objective heating collar using $488 \mathrm{~nm}$ and $543 \mathrm{~nm}$ laser excitation with identical offset, gain and detector settings for each time point. The nonentry time point ( $\mathrm{t}-0$ ) was examined following addition of $2 \mathrm{ml}$ of ice-cold Opti-MEM I on a stage at ambient temperature. To reduce problems of sample photobleaching, separate plates were examined for each of the time points ( $t-10, t-15, t-20, t-25$, and $t-30)$.

Synchronized fusion assay. To prevent the acidification of endosomes and synchronize fusion of virions with endosomal membranes we used the lysosomotropic reagent $\mathrm{NH}_{4} \mathrm{Cl}$ as described previously (174) with some modifications. BHK-21 cells on coverslips were washed twice with PBS and then washed twice with PBS containing $100 \mathrm{mM} \mathrm{NH}_{4} \mathrm{Cl}$. Viruses (MOI 50-100) either with or without $50 \mu \mathrm{g}$ of transferrin-TR were adsorbed in SF-DMEM containing $100 \mathrm{mM} \mathrm{NH}_{4} \mathrm{Cl}$ for 60 or 90 minutes. After adsorption the cells were washed with PBS four times to remove the $\mathrm{NH}_{4} \mathrm{Cl}$ and were either fixed with $3 \%$ PFA (t-0), or $2 \mathrm{ml}$ of SF-DMEM at $37^{\circ} \mathrm{C}$ containing $10 \mu \mathrm{g} / \mathrm{ml}$ cycloheximide was added for 60 minutes (t-60) and then fixed. Synchronized fusion assays using the cytoskeletal inhibitors cytochalasin D or nocodazole were performed as above, except virus was adsorbed for 60 minutes in media with $100 \mathrm{mM} \mathrm{NH}_{4} \mathrm{Cl}$ and the medium was replaced with SF-DMEM containing $10 \mu \mathrm{M}$ of the inhibitor and $100 \mathrm{mM}$ $\mathrm{NH}_{4} \mathrm{Cl}$ and incubated for 30 minutes. The $\mathrm{NH}_{4} \mathrm{Cl}$ was then washed out as described above but the cytoskeletal inhibitors remained for 60 or 120 minutes post lysosomotropic washout (t-60 or 120) and the cells were fixed with 3\% PFA and prepared for IF.

\section{Results}

\section{Recovery and characterization of recombinant VSV encoding fluorescent M}

proteins. To investigate virus uncoating and the fate of released $\mathrm{M}$ protein we constructed two different types of $\mathrm{M}$ proteins with fluorescent tags. The first type consisted of green fluorescent protein (GFP) fused to the C-terminus of M (Fig. 2-1A, MFC). Three different MFC constructs were generated with linkers of $8\left(\mathrm{Gly}_{3}-\mathrm{Ser}\right)_{2}$ 
(MFC-II), 15 (Gly ${ }_{4}$-Ser) $)_{3}$ (MFC-III), and 20 (Gly 4 -Ser) $)_{4}$ (MFC-IV) amino acids. Previously others had reported the construction of M-GFP fusion proteins with GFP fused at either the N- $(69,168,204)$ or C-terminus (123) of M, but none of these contained a linker separating $\mathrm{M}$ and GFP, which we reasoned may be important for retaining the assembly function of $\mathrm{M}$ protein. We chose to fuse GFP at the C-terminus to minimize interference with membrane association, which maps to the N-terminus of $\mathrm{M}$ $(30,31)$. All three MFC proteins had identical intracellular distribution patterns when examined by immunofluorescence microscopy (IF) which was indistinguishable from $\mathrm{M}$ wild-type (wt) distribution (Fig. 2-2A).

To determine if the three MFC constructs retained the ability to bud vesicles when expressed in cells (97) the amount of $\mathrm{M}$ released into the supernatant was examined. Despite the normal intracellular distribution of the three MFC proteins, there was a significant reduction in the amount of ${ }^{35} \mathrm{~S}$-methionine labeled protein in supernatants from cells expressing the MFCs (MFC-III shown) compared to cells expressing Mwt (Fig. 2-2B lanes 3 and 2, respectively). To determine if the reduced budding activity was due to a large C-terminal extension or was a specific GFP effect on budding activity, we fused glutathione-S-transferase (GST) to the C-terminus of M (M-GST). Similar to the results with the MFC proteins, we observed a large reduction in budding activity (Fig. 22B lane 4) even though there was no difference in cellular distribution of M-GST when compared to Mwt (data not shown).

The low level budding activity of MFC and M-GST suggested that these proteins might not be able to support virus assembly. Indeed, multiple attempts to recover rVSV expressing any of the three MFC proteins or M-GST as the sole M protein were unsuccessful ( $n=6$ per construct). However, we did recover rVSVs that expressed both Mwt and the MFC proteins (rVSV-M-MFC, -II, -III, and -IV) from the same genome (Fig. 2-1A). Characterization of these viruses showed that the growth kinetics were similar to rVSV-wt (Fig. 2-2C, rVSV-M-MFC-III shown), however it was found that MFC-III was incorporated at only 5\% when compared to the amount of Mwt in rVSV-MMFC-III virions (Fig. 2-2D lane 4). When virions were solubilized with Triton X-100 and the RNP and envelope fractions examined, MFC-III was found mainly in the RNP pellet fraction (Fig. 2-2D lanes 5 and 9). This result was unexpected since MFC-III was seen at the plasma membrane of cells infected with rVSV-M-MFC-III (Fig. 2-2E, arrows). To determine if the small amount of MFC-III incorporated into virions was enough to detect in an uncoating assay, purified virus was bound to cells in the cold at a multiplicity of 100. Cells were washed to remove unbound virus, fixed with paraformaldehyde and then examined by fluorescence microscopy. Although virions could be detected when the fixed cells were permeabilized and probed with an $\mathrm{M}$ antibody, no GFP signal could be detected (data not shown). Considering the low amount of MFC incorporation and lack of GFP signal when virions were bound to cells, it was necessary to use an alternative strategy to make rVSV with fluorescent M protein.

The second type of fluorescent $\mathrm{M}$ protein we generated utilized Lumio ${ }^{\mathrm{TM}}$ technology, in which a tetracysteine (-CCRECC-) Lumio $^{\mathrm{TM}}$ tag (Fig. 2-1B) was fused to the $\mathrm{N}$ or $\mathrm{C}$-terminus of $\mathrm{M}$ (Lumio-M and M-Lumio, respectively). To assess the cellular 
Figure 2-2. Characterization of the MFC protein and rVSV-M-MFC virions. (A) Laser scanning confocal microscopy of GFP, M-wt (Wt), and MFC-III expressed in BHK-21 cells. Images are from $1 \mu \mathrm{m}$ optical sections near the center of the cell where GFP was detected using laser excitation at $488 \mathrm{~nm}$ and $M$ by staining with a $\mathrm{M} \mathrm{mAb}$ conjugated to rhodamine using $543 \mathrm{~nm}$ excitation. (B) M protein budding assay. Cell extracts and supernatants from cells infected with vTF7-3 alone (lane 1), or expressing Mwt (lane 2), MFC-III (lane 3), or M-GST (lane 4) were immunoprecipitated with M $\mathrm{mAb}$ after a 16 hour ${ }^{35} \mathrm{~S}$-methionine labeling and examined by SDS-PAGE. (C) Growth kinetics of rVSV-M-MFC and rVSV-wt. Aliquots of media were collected at the indicated times post infection and titers were determined by plaque assay on BHK-21 cells. (D) Immunoblots of purified rVSV-wt (Wt) and rVSV-M-MFC (M-MFC) virus (lanes 1 and 4) using a monoclonal anti-M antibody, or RNP (lanes 2 and 7, lanes 5 and 9) and TCA precipitated supernatants (lanes 3 and 8, lanes 6 and 10), after Triton X-100 solubilization of virus and ultracentrifugation over a $20 \%$ sucrose cushion, using anti-M and anti-GFP monoclonal antibodies. (E) Localization of MFC in BHK-21 cells infected with rVSV-M-MFC observed by confocal microscopy using laser excitation at 488nm at 8 hours post infection. Arrows show MFC associating with the plasma membrane of cells. 
A
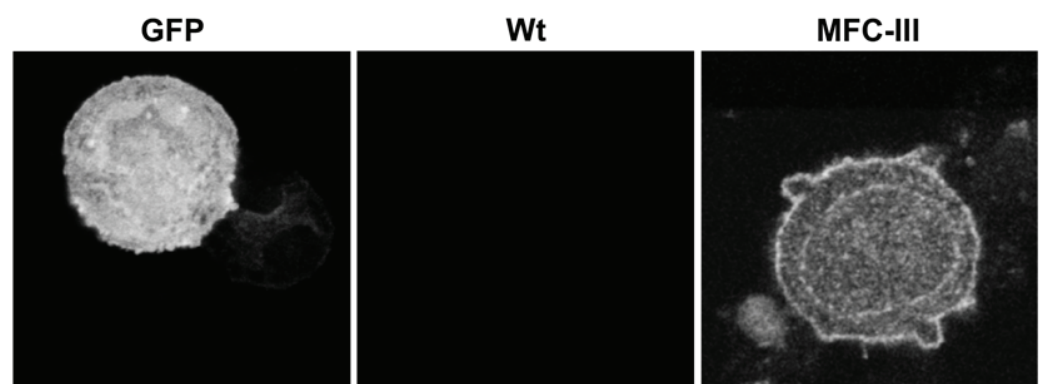

ex. $488 n m$
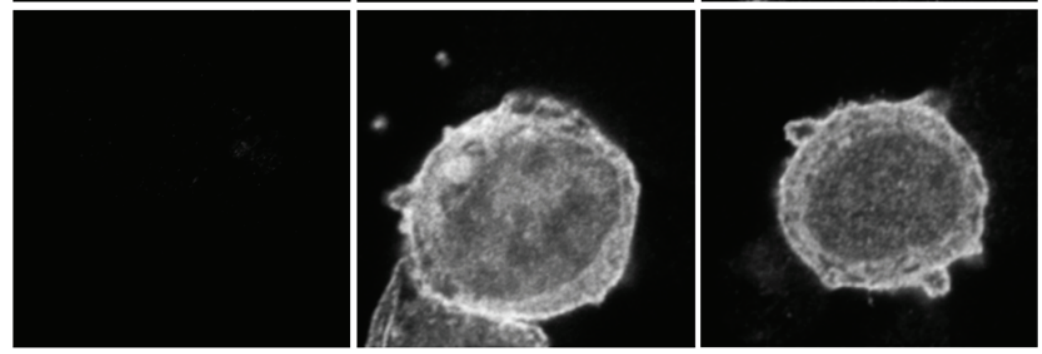

ex. $543 \mathrm{~nm}$

B

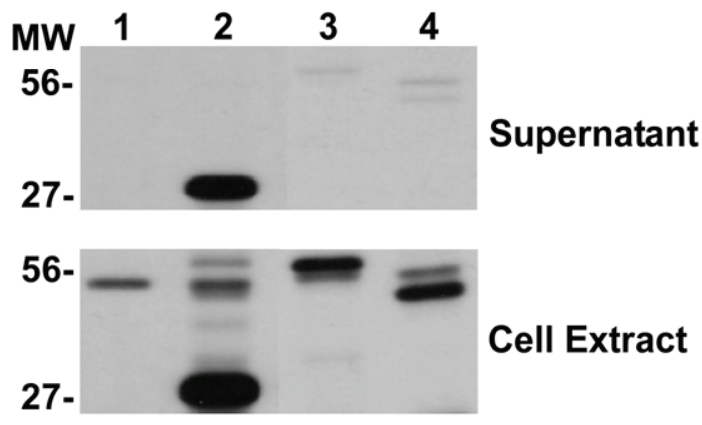

C

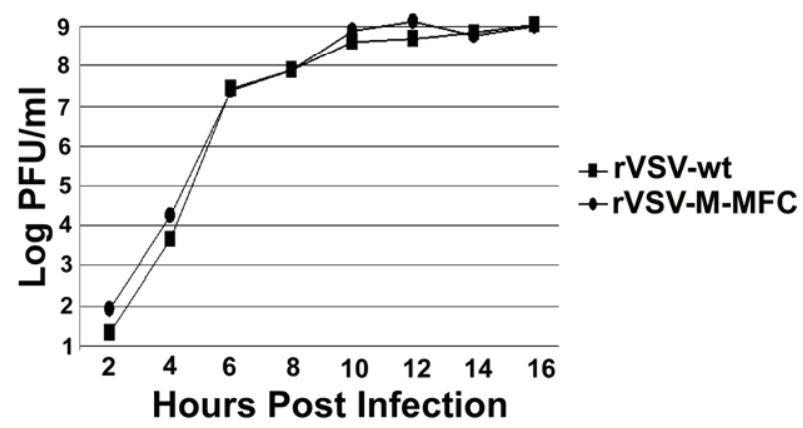

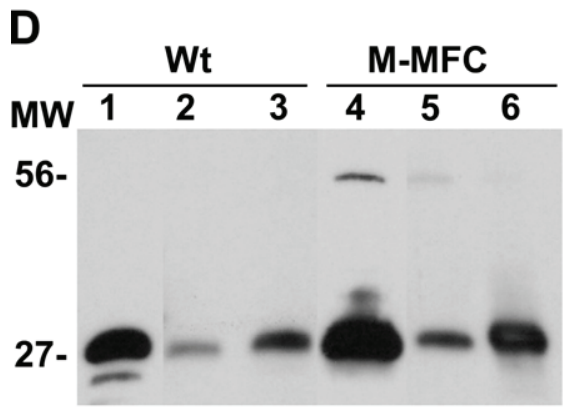

anti-M

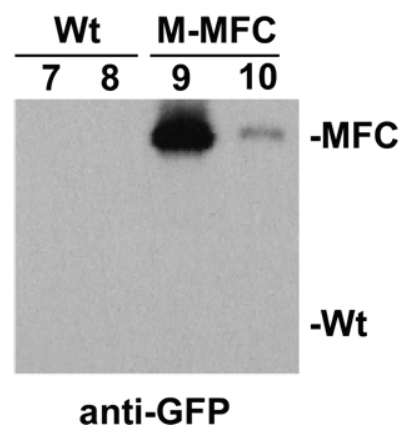

E

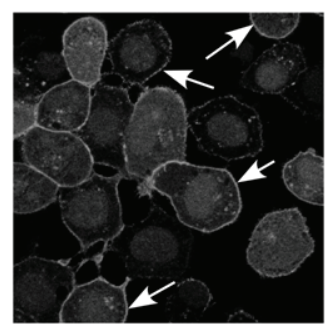


distribution and assembly function of these tagged proteins we compared them to $\mathrm{M}$-wt by IF (Fig. 2-3A) and in a budding assay (Fig. 2-3B). There was no discernable difference between the distributions of the $M$ proteins as they all were located in the nucleus, were cytosolic, and localized to the plasma membrane (Fig. 2-3A). When examined for budding activity, the N-terminally tagged Lumio-M showed a reduction in the amount of protein released from cells (Fig. 2-3B lane 4) although not to the extent of the MFC protein (Fig. 2-2B lane 3). The C-terminally tagged M-Lumio on the other hand had wild-type budding activity (Fig 2-3B lane 6).

To determine if Lumio-M or M-Lumio could support virus assembly we replaced the Mwt gene with the Lumio tagged versions in the viral genome (Fig. 2-1B). Both viruses were easily recovered demonstrating that $\mathrm{M}$ proteins with small $\mathrm{N}$ or $\mathrm{C}$ terminal tags are functional for virus replication and assembly. However, there was a growth defect observed for the N-terminally tagged Lumio-M virus whereas the growth kinetics of the virus with the C-terminally tagged M-Lumio virus was similar to rVSV-wt (Fig. 2$3 \mathrm{C})$. Because the $\mathrm{C}$-terminally tagged $\mathrm{M}$-Lumio virus had growth properties indistinguishable from rVSV-wt we used the rVSV-M-Lumio construct to make fluorescent rVSV and for the uncoating studies described below.

Fluorescent labeling of rVSV-M-Lumio. Lumio ${ }^{\mathrm{TM}}$ Green is a small (MW 664.5), membrane permeable fluorophore which fluoresces green when covalently bound to the thiol pairs in the Lumio $^{\mathrm{TM}}$ tag (Fig. 2-1B, *) $(2,64,70)$. To fluorescently label the rVSV-M-Lumio virus, we initially mixed purified virus with the Lumio ${ }^{\mathrm{TM}}$ Green reagent similar to that described by others to label HIV virions encoding a Lumio-tagged integrase protein (5). However, in our case no labeling occurred suggesting that the Lumio $^{\mathrm{TM}}$ tag was not available, or incapable of interacting with the fluorophore within virions. We reasoned that the Lumio $^{\mathrm{TM}}$ tag may be more accessible for labeling within cells before assembly into M-Lumio virions, therefore we added Lumio ${ }^{\mathrm{TM}}$ Green to the media of cells infected with rVSV-M-Lumio at 5 hours post infection (hpi). The fluorophore containing media was removed one hour later and the cells were examined by IF (Fig. 2-4A). We observed that the M-Lumio protein bound the fluorophore and the labeling colocalized with $\mathrm{M}$ protein staining (Fig. 2-4A, merge). To produce fluorescent virus, the fluorophore was added to infected cells at 4 hpi and the virus in the media was harvested at $18 \mathrm{hpi}$. The labeled virus (rVSV-M-Lumio-Green) was concentrated by ultracentrifugation through a sucrose cushion, resuspended in Tris-saline sucrose, and passed through Sephadex G-50 columns to separate labeled virions from residual free Lumio $^{\mathrm{TM}}$ reagent. The purified virions were titered and the amount of fluorescence determined using a fluorometer. To examine relative infectivity of the labeled rVSV-MLumio-Green to a similarly prepared batch of rVSV-wt, we compared the protein profiles from $1 \times 10^{7}$ PFU of rVSV-wt and rVSV-M-Lumio-Green after electrophoresis on SDSpolyacrylamide gels (Fig. 2-4B, left-hand gel). The slightly slower migration of MLumio compared to Mwt is due to the additional 17 amino acids of the Lumio ${ }^{\mathrm{TM}}$ tag. Coomassie-blue staining indicated the two virus preparations had similar amounts of protein, but the rVSV-M-Lumio-Green had a 60-fold higher fluorescence signal than rVSV-wt virus. To determine whether the fluorescent signal from the virions was due to 
Figure 2-3. Characterization of Lumio-M and M-Lumio and rVSV-MLumio/(Lumio-M) virions.

(A) Laser scanning confocal microscopy of $\mathrm{M}$ proteins expressed in BHK-21 cells and stained with monoclonal anti-M antibody conjugated to rhodamine. (B) M protein

budding assay. Cell extracts (lanes 1, 3, and 5) and supernatants (lanes 2, 4, and 6) from cells expressing Mwt, Lumio-M, and M-Lumio after a 16 hour ${ }^{35}$ S-methionine labeling, immunoprecipitation with $\mathrm{M} \mathrm{mAb}$, and analysis by SDS-PAGE. (C) One-step growth curve of rVSV-wt, rVSV-Lumio-M, and rVSV-M-Lumio. 
A

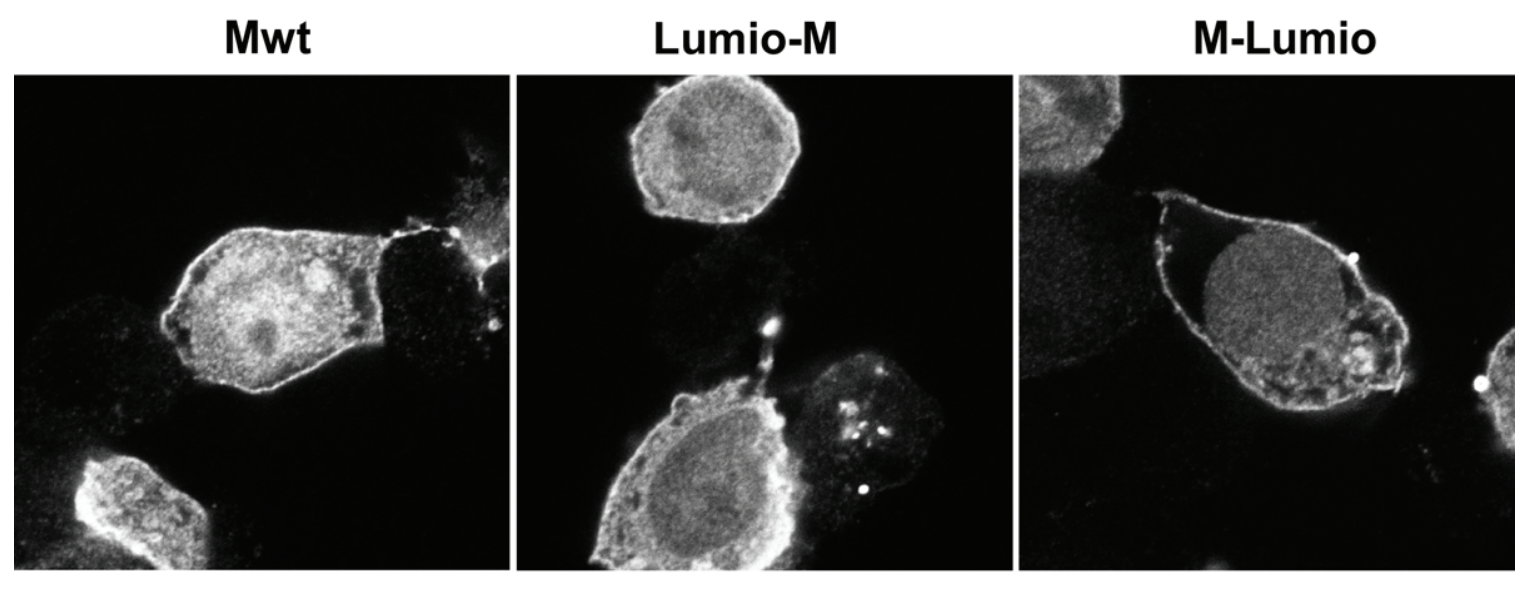

B

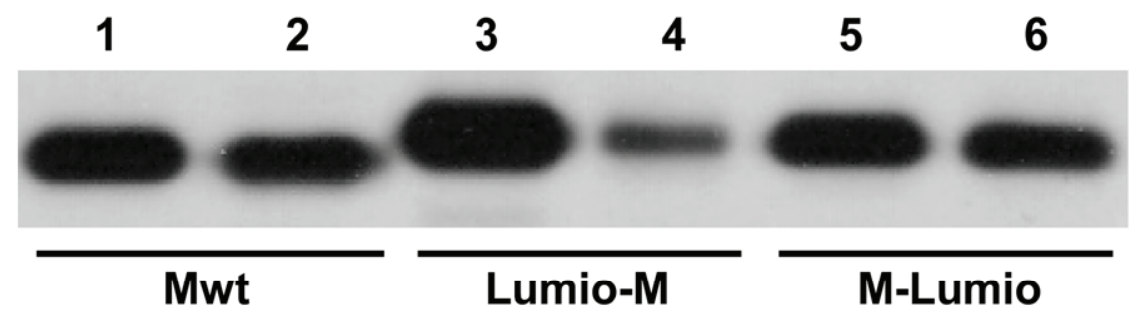

C

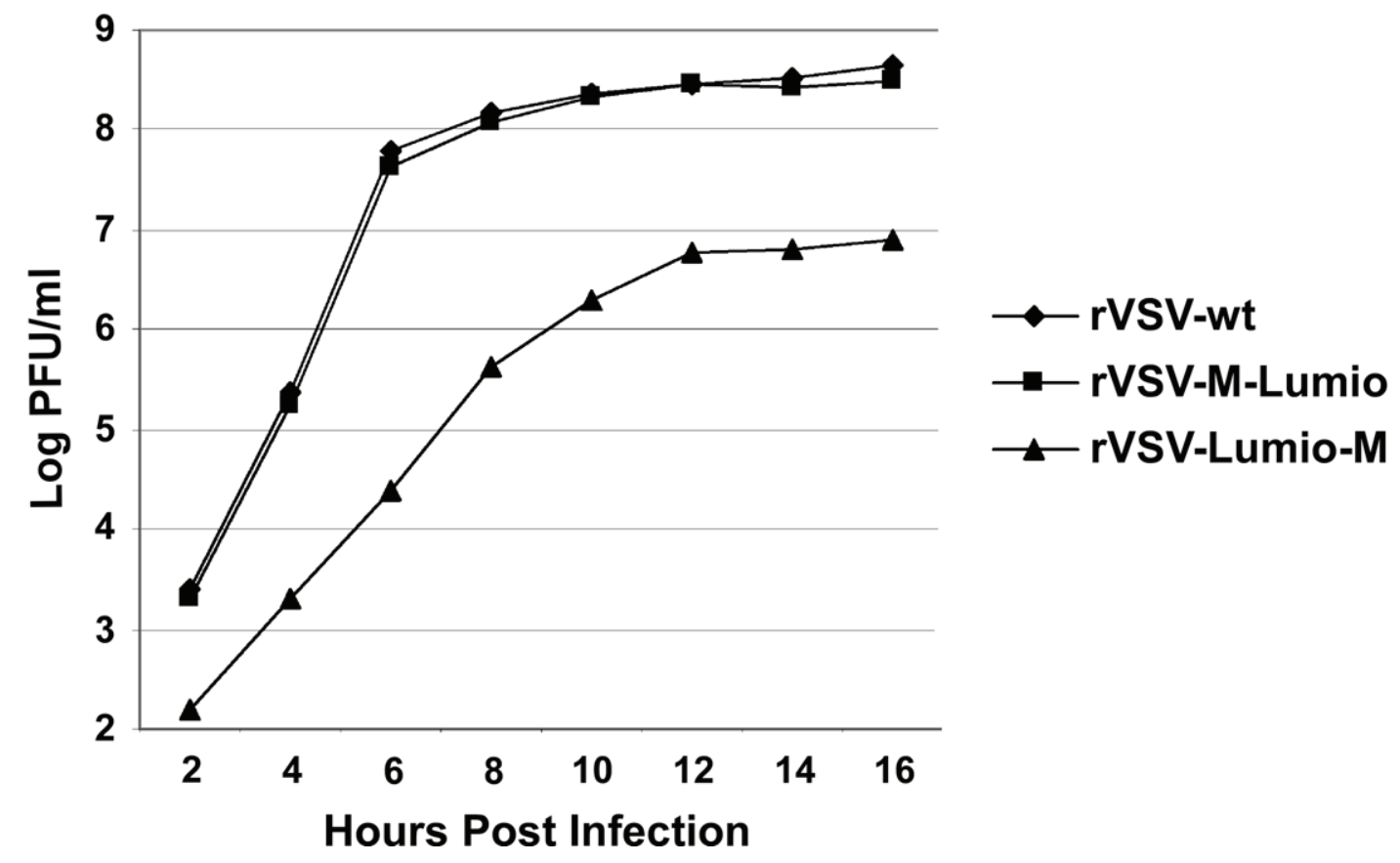




\section{A}
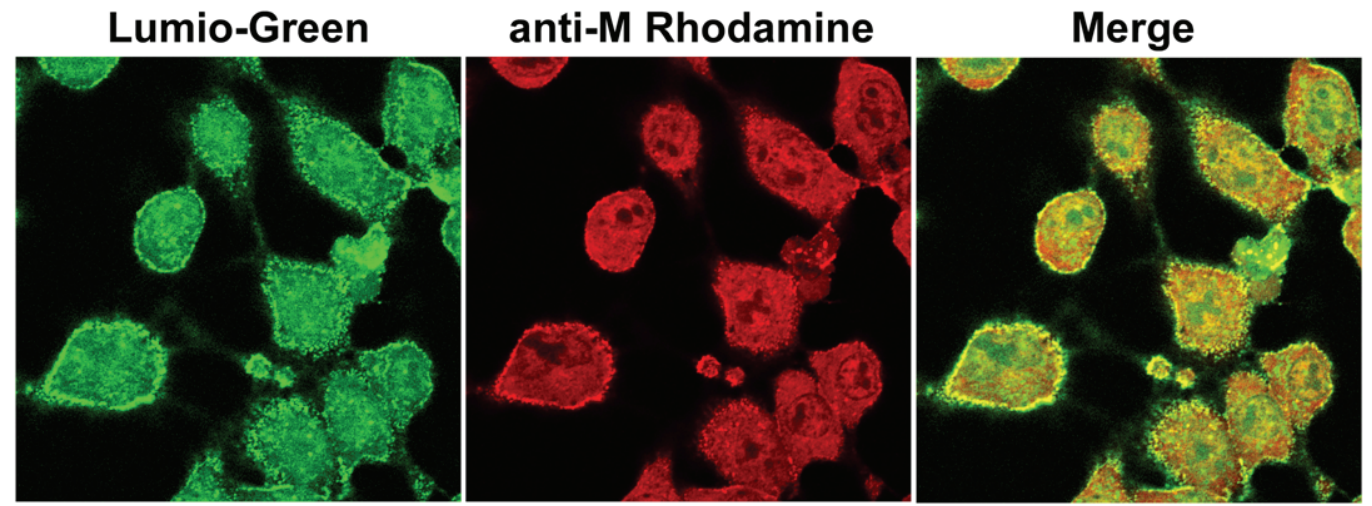

$6 \mathrm{hpi}$

B

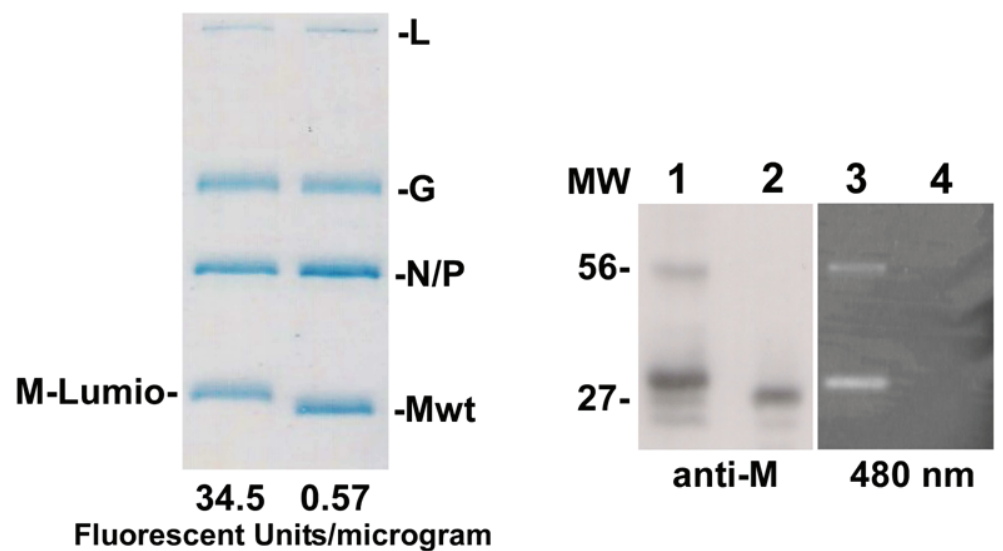

Figure 2-4. Labeling of rVSV-M-Lumio with Lumio ${ }^{\mathrm{TM}}$ Green.

(A) Laser scanning confocal microscopy of BHK-21 cells infected with rVSV-M-Lumio and labeled with Lumio ${ }^{\mathrm{TM}}$ Green reagent for 1 hour at 6 hours post infection. (B) SDSPAGE analysis of $10^{7}$ PFU rVSV-M-Lumio-Green and rVSV-wt virions. The left-hand gel is stained with Coomassie blue. The relative fluorescence signal $/ \mu \mathrm{g}$ is shown below each lane. The center gel is an immunoblot for $\mathrm{M}$ (anti-M, lanes 1 and 2), and the righthand gel (lanes 3 and 4) was visualized by excitation at 480nm on a STORM phosphorimager. Lanes 1 and 3 contain rVSV-M-Lumio and lanes 2 and 4 contain rVSV-wt. The anti-M reactive and fluorescent bands at approximately $56 \mathrm{KDa}$ are likely artificially induced M-Lumio dimers formed as a result of the reduced concentration of $\beta$ mercaptoethanol used in the sample buffer (see Methods for details). 
specific binding of the Lumio $^{\mathrm{TM}}$ reagent to the M-Lumio protein, we used a STORM fluorescence imager (Molecular Dynamics) to visualize fluorescent proteins from purified virus after separation by SDS-PAGE. Fluorescent protein bands were only observed with the rVSV-M-Lumio virus and the bands that corresponded to fluorescent M-Lumio protein were also detected in an immunoblot using an M-specific antibody (Fig. 2-4B lanes 1 and 3, respectively). A higher molecular weight band was seen also in the M-Lumio lanes by fluorescence and immunoblot analysis. This band corresponded to an M-Lumio dimer and was the result of two conditions: i) the reduced amount of $\beta$ mercaptoethanol in the protein sample buffer, which was required to retain the Lumio ${ }^{\mathrm{TM}}$ Green on the M-Lumio protein since increased reducing conditions results in the loss of Lumio-Green binding (data not shown); and ii) the addition of four cysteines to $\mathrm{M}$ (wildtype has one cysteine at residue 135). Overall, these data demonstrated that rVSV-MLumio-Green was specifically labeled on the M protein and therefore should be useful to examine virus uncoating and fate of M-Lumio-Green within cells.

Live-cell entry of rVSV-M-Lumio-Green. To follow the entry of rVSV-MLumio-Green virions into live cells, we adsorbed the virus (MOI 50) at $4^{\circ} \mathrm{C}$ for 90 minutes to prevent endocytosis of virions, and then initiated their entry by endocytosis after addition of media at $37^{\circ} \mathrm{C}$. Transferrin conjugated to Texas Red (transferrin-TR) was used as an early and recycling endosomal marker during endocytosis of rVSV-MLumio-Green and both reagents were followed in 5 minute intervals over a 30 minute time span, post addition of warm media (Fig. 2-5). Images for each time point were acquired using identical detector settings to allow changes in fluorescence intensity to be quantified. Figure 2-5 shows representative images of rVSV-M-Lumio-Green and transferrin-TR at t-0 (top panels), t-5 (middle panels), and t-20 (bottom panels). At t-0, rVSV-M-Lumio-Green and transferrin-TR were bound to the plasma membrane and showed some colocalization. At t-5 (Fig. 2-5, t-5) both markers were internalized, presumably in early endosomes. At t-20 (Fig. 2-5, t-20), the bulk of M-Lumio-Green remained associated with perinuclear endosomes that colocalized with transferrin-TR (Fig. 2-5, t-20 merge), while a fraction of M-Lumio-Green was found at what appeared to be the nuclear envelope (Fig. 2-5, t-20 arrows). M-Lumio-Green could be detected on the nuclear envelope as early as t-10 (data not shown), with increasing intensity up to t20 , but there was no apparent gain in intensity of M-Lumio-Green fluorescence at the nuclear envelope after 20 minutes post-entry. At no time did we observe transferrin-TR associated with the nuclear envelope.

Synchronized fusion assay with rVSV-M-Lumio-Green. To examine the role of new viral protein synthesis on the distribution of M-Lumio-Green after uncoating, we synchronized viral fusion in the presence of cycloheximide as was done in a previous study (174) with some modifications. rVSV-M-Lumio-Green virions (MOI 50) were adsorbed to cells in the presence of $\mathrm{NH}_{4} \mathrm{Cl}$ to prevent acidification of endosomes and thereby inhibiting fusion of endocytosed virions $(82,174)$. The inoculum and the lysosomotropic agent were removed, the cells washed 4 times, and media containing cycloheximide was added. Cells were then fixed at varying times post $\mathrm{NH}_{4} \mathrm{Cl}$ removal, 


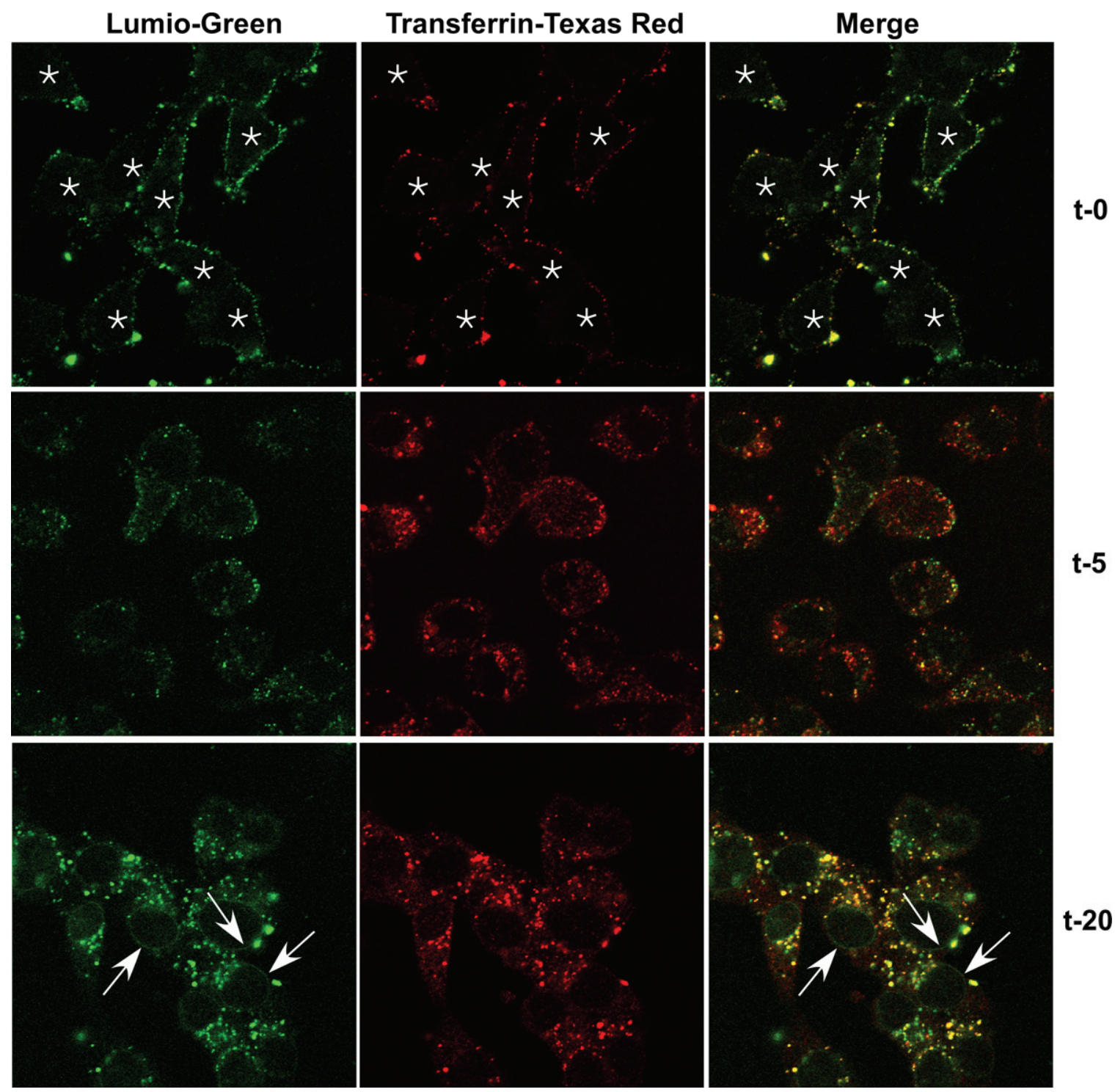

Figure 2-5. Synchronized entry assay with rVSV-M-Lumio-Green.

Cells were inoculated with rVSV-M-Lumio-Green and transferrin-TR at $4^{\circ} \mathrm{C}$ for 90 minutes, and either fixed immediately with ice-cold $3 \%$ paraformaldehyde $(\mathrm{t}-0)$ or were quickly warmed by the addition of $37^{\circ} \mathrm{C}$ media and examined by laser scanning confocal microscopy. Images were collected every 5 minutes for 20 minutes using separate $35 \mathrm{~mm}$ glass bottomed dishes for each time point to reduce loss of signal by photobleaching. (Top panels) Representative images of M-Lumio-Green and transferrin-TR at t- 0 where cells were fixed at $4^{\circ} \mathrm{C}$, * represents the center of a cell. (Middle panels) Images collected at 5 minutes ( $\mathrm{t}-5)$ post addition of warm media. (Bottom panels) Images taken at 20 minutes ( $\mathrm{t}-20)$ after warm-up. Note the localization of M-Lumio-Green at the nuclear envelope (arrows) and in perinuclear endosomes that colocalizes with transferrin. Magnification 100X. 
stained with Nup62 antibody which binds to the nuclear pore complex (NPC) on the nuclear envelope, and with an $\mathrm{M}$ mAb, and observed by IF. At t-0, rVSV-M-LumioGreen particles were in peripheral endosomes, similar to that found at the t- 5 time point in the live-cell synchronized entry assay (Fig. 2-6, t-0). At t-60, the bulk of M-LumioGreen was found in perinuclear endosomes (Fig. 2-6, t-60) and at the nuclear envelope (arrows). Similar results were observed for rVSV-wt infected cells that were stained using the $\mathrm{M} \mathrm{mAb}$ (data not shown) except nuclear envelope staining was not detected by the $\mathrm{M} \mathrm{mAb}$ in either case (Fig. 2-6, t-60 center image) as this antibody binds to the site on $\mathrm{M}$ required to bind at the NPC (168). Delivery of M-Lumio-Green to the nuclear envelope was confirmed by colocalization with the nucleopore protein Nup62 (Fig. 2-6, t60 merge). These data showed that after $\mathrm{NH}_{4} \mathrm{Cl}$ washout $\mathrm{M}$-Lumio-Green localized to the nuclear envelope in the absence of new protein synthesis and therefore represents $\mathrm{M}$ protein that is released into the cytoplasm and trafficked to the nucleus after uncoating.

The perinuclear endosomes associated with M-Lumio-Green colocalize with transferrin-TR. M-Lumio-Green distribution was perinuclear by 15 to 20 minutes post infection during synchronized entry of virus and at time points past t-20 (data not shown) of the synchronized fusion assay. To determine the identity of these perinuclear structures, we used markers that corresponded to lysosomes (LAMP-1), late endosomes/lysosomes (Mannose-6-Phosphate receptor, M-6-P-R), and early/recycling endosomes (transferrin-TR). We performed synchronized fusion assays with rVSV-MLumio-Green, as described above, and used monoclonal antibodies to label the cells for LAMP-1 (Fig. 2-7A) and M-6-P-R (Fig. 2-7B), or visualized directly for transferrin-TR (Fig. 2-7C). While some M-Lumio-Green colocalized with LAMP-1 (Fig. 2-7A) and M6-P-R (Fig. 2-7B) the majority of M-Lumio-Green colocalized with transferrin-TR (Fig. 2-7C). When images of the transferrin-TR endosomes that colocalized with M-LumioGreen were magnified (500X) the M-Lumio-Green was not evenly distributed throughout the endosome, but instead appeared to be located on one side of the endosome (Fig. 2-7D arrows). A similar distribution of M-Lumio-Green and transferrin-TR was observed for images from the t-20 time point for synchronized entry (Fig. 2-5, t-20), which suggested the results seen in figure $2-7 \mathrm{C}$ were not an artifact of lysosomotropic reagent treatment. In contrast to the asymmetric distribution of rVSV-M-Lumio-Green in endosomes at $\mathrm{t}-60$, internalized $\Delta$ G-rVSV-M-Lumio-Green virions, which lack $\mathrm{G}$ protein and therefore cannot fuse with the endosomal membrane, had a more diffuse distribution within the transferrin-TR positive endosomes (Fig. 2-7E), suggesting the asymmetric localization of M-Lumio-Green may represent virus that has fused and released its cargo. These data indicate that even after 60 minutes most of the virus is not trafficked to late endosomes and lysosomes, but remains in compartments that are thought to be the primary site of virus uncoating.

Trafficking of M-Lumio-Green to perinuclear endosomes and the nuclear envelope does not require actin or tubulin. To determine whether or not actin and tubulin were required for delivery of M-Lumio-Green to the perinuclear endosomes at t60 or were required for delivery of M-Lumio-Green to the nuclear envelope, we treated 

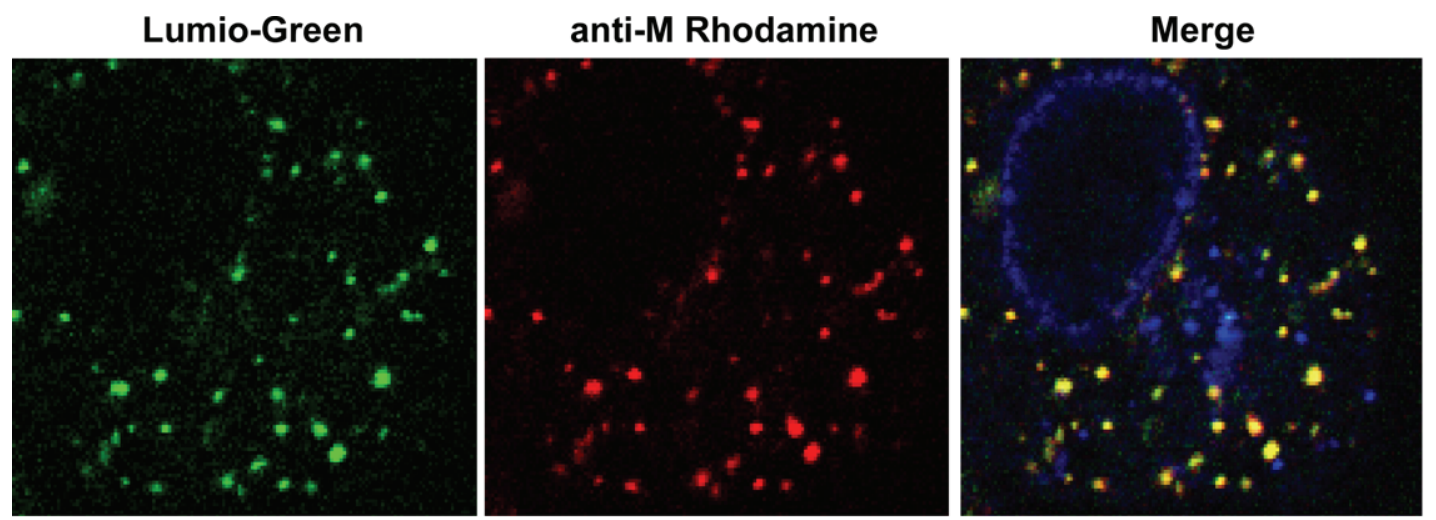

t-0
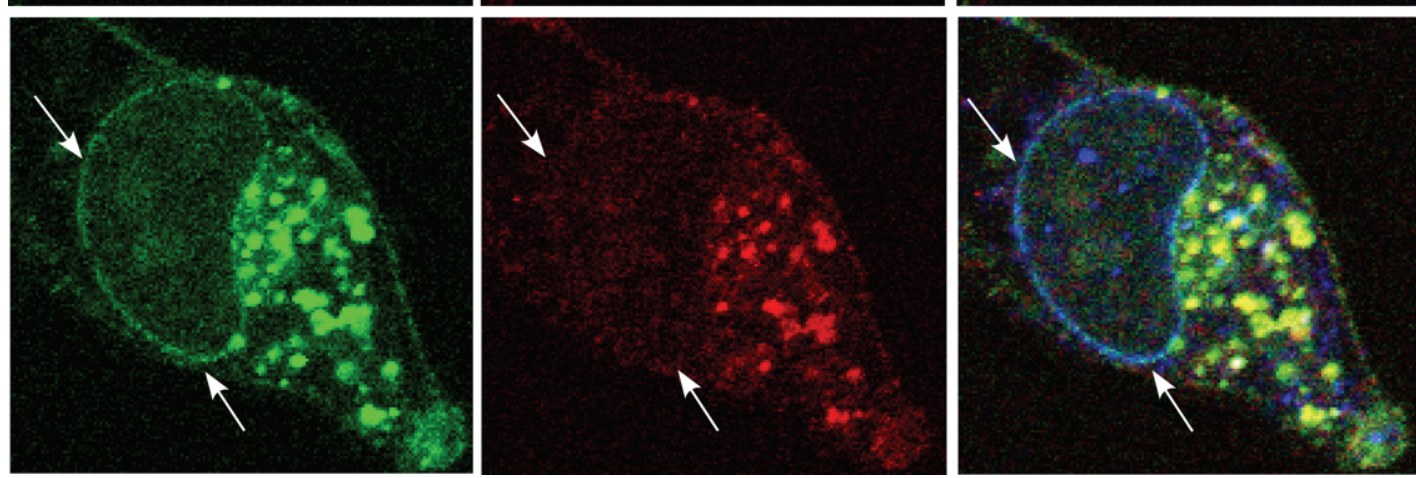

Figure 2-6. Synchronized fusion assay with rVSV-M-Lumio-Green.

BHK cells were inoculated with rVSV-M-Lumio-Green at MOI 50 in the presence of $\mathrm{NH}_{4} \mathrm{Cl}$ for 90 minutes to allow accumulation of virus in early endosomes. Entry was initiated by incubation in media without $\mathrm{NH}_{4} \mathrm{Cl}$, but containing cycloheximide. Cells were fixed at $\mathrm{t}-0$ (top panels) and $\mathrm{t}-60$ (bottom panels), stained for $\mathrm{M}$ protein using a rhodamine conjugated anti-M mAb (red) and Nup62 labeled with Alexa 647 (blue), and examined by laser scanning confocal microscopy. Arrows indicate the localization of MLumio-Green at the nuclear envelope. Note the $\mathrm{M} \mathrm{mAb}$ does not detect $\mathrm{M}$ protein localized to the nuclear envelope. Magnification 200X. 
Figure 2-7. Identification of perinuclear structures associated with M-Lumio-Green post fusion.

A synchronized fusion assay was performed as described for figure 2-6. Cells were fixed at t-60 then stained for (A) LAMP1, (B) Mannose-6-Phosphate Receptor, or (C)

Transferrin-Texas Red and examined by confocal microscopy at $100 \mathrm{X}$ magnification. (D) is at 500X magnification of perinuclear vesicles containing M-Lumio-Green and transferrin-TR. Arrows indicate an asymmetric distribution of M-Lumio-Green within transferrin-TR positive endosomes. (E) 500X magnification image of $\Delta \mathrm{G}-\mathrm{rVSV}-\mathrm{M}-$

Lumio-Green inoculated cells showing transferrin-TR positive endosomes with a uniform distribution of M-Lumio-Green within endosomes. 
A
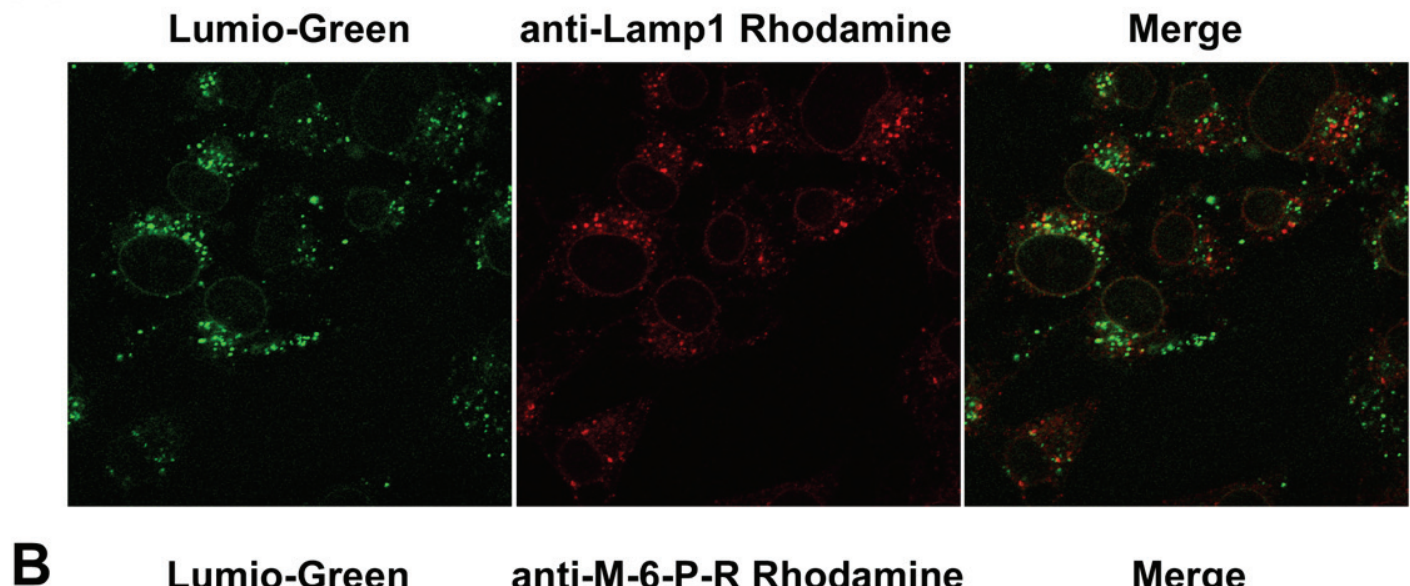

Merge

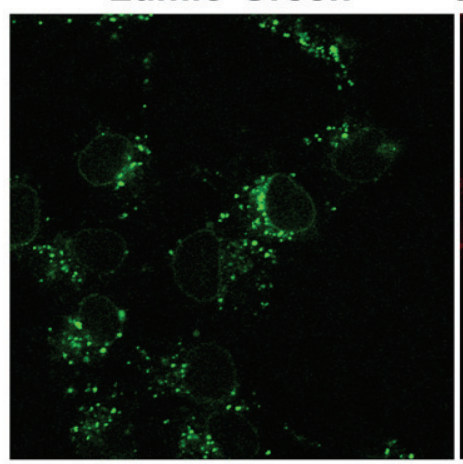

anti-M-6-P-R Rhod

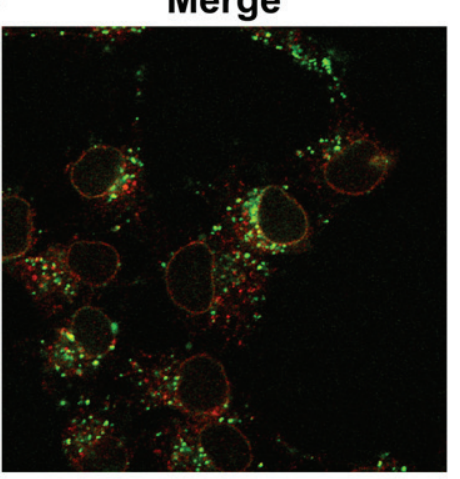

C

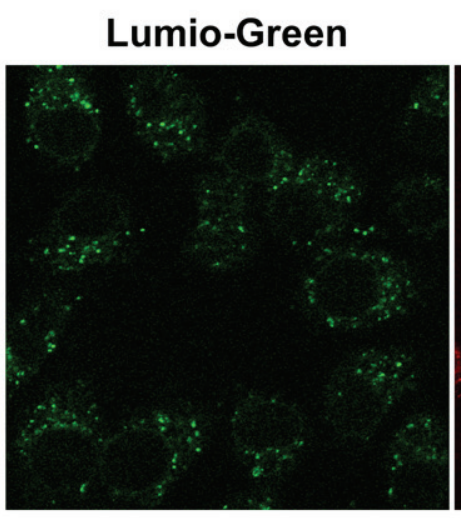

Transferrin-Texas Red

Merge

D

rVSV-M-Lumio-Green

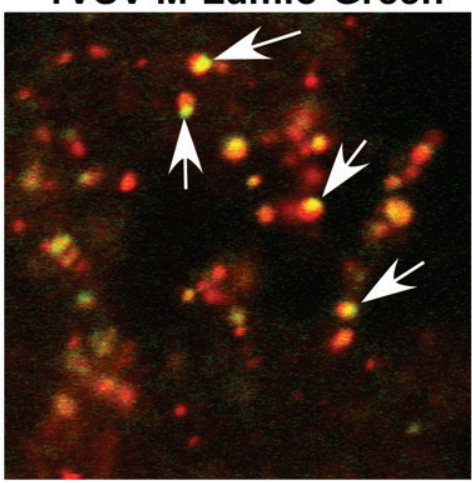

E

$\Delta$ G-rVSV-M-Lumio-Green 
cells with the cytoskeletal inhibitors cytochalasin D or nocodazole in a modified synchronized fusion assay. Cells were inoculated with virions in the presence of $\mathrm{NH}_{4} \mathrm{Cl}$ for 60 minutes, and then the cytoskeletal inhibitors were added for the last 30 minutes of a 90 minute incubation. After the 90 minutes of accumulation in early endosomes, the cells were washed with media that either did or did not contain the cytoskeletal inhibitors, and that also contained cycloheximide. At t-60 the cells were fixed and stained for filamentous actin, using Texas Red-X phalloidin (Fig. 2-8) or for tubulin (Fig. 2-9). Either in the absence (Fig. 2-8, top panels) or presence of cytochalasin D (Fig. 2-8, bottom panels) after $\mathrm{NH}_{4} \mathrm{Cl}$ wash out M-Lumio-Green was found in perinuclear endosomes and at the nuclear envelope (Fig. 2-8, arrows). When cells were treated with nocodazole to determine the involvement of microtubules in M-Lumio-Green distribution to the perinuclear region and nuclear envelope, we observed a reduction in the tight perinuclear localization of endosomes containing M-Lumio-Green (Fig. 2-9, top versus bottom panels), but the treatment did not prevent the association of M-Lumio-Green with the nuclear envelope (Fig.2-9, arrows bottom panels). These results suggest that delivery of M-Lumio-Green to the nuclear envelope does not require intact actin or microtubules.

\section{Decrease in colocalization of M-Lumio-Green and $\mathrm{N}$ correlates with} productive infection and does not require tubulin. To determine if the M-LumioGreen nuclear envelope distribution was a result of uncoating, we assessed whether or not we could detect M-Lumio-Green physically separating from nucleocapsids by observing a decrease in colocalization of M-Lumio-Green and $\mathrm{N}$ using $\mathrm{N}$ mAb conjugated to rhodamine in the presence of nocodazole and cycloheximide. rVSV-M-Lumio-Green (MOI 100) was endocytosed into cells in the presence of $\mathrm{NH}_{4} \mathrm{Cl}$ and nocodazole and the cells were observed at t- 0 (Fig. 2-10A) and t-120 post $\mathrm{NH}_{4} \mathrm{Cl}$ washout, either in the presence of nocodazole and cycloheximide (Fig. 2-10B), or in media that contained only nocodazole (Fig. 2-10C). At t-0, Figure 2-10A shows there was $92.8 \%(+/-3.4 \%, \mathrm{n}=50$ cells) colocalization of $\mathrm{M}$ and $\mathrm{N}$, whereas at t-120 (Fig. 2-10B) most of the $\mathrm{M}$ had separated from the nucleocapsids as indicated by only $31.3 \%(+/-8.2 \%, \mathrm{n}=50$ cells) colocalization (Fig. 2-10B). As previously reported by others (42), we also observed that rVSV-M-Lumio-Green established a productive infection without an organized microtubule network (Fig. 2-10C). These results suggest that the physical separation of M-Lumio-Green from N protein observed by confocal microscopy represents the end result of an uncoating event which can lead to a productive infection, and that this uncoating event does not require an intact microtubule network.

\section{Discussion}

Recently visualization within live cells of Ebola VP40 (164) and HIV-1 integrase (5) has been reported using a fluorescent Lumio ${ }^{\mathrm{TM}}$ technology developed within the last decade $(2,70)$. Fluorescently tagged viral proteins using GFP to study entry dynamics and intracellular trafficking of $\operatorname{HIV}-1(25,152)$ and VSV $(41,42,202)$ have also been used. Although the G $(41,202)$ and P (42) proteins of VSV have been fluorescently 

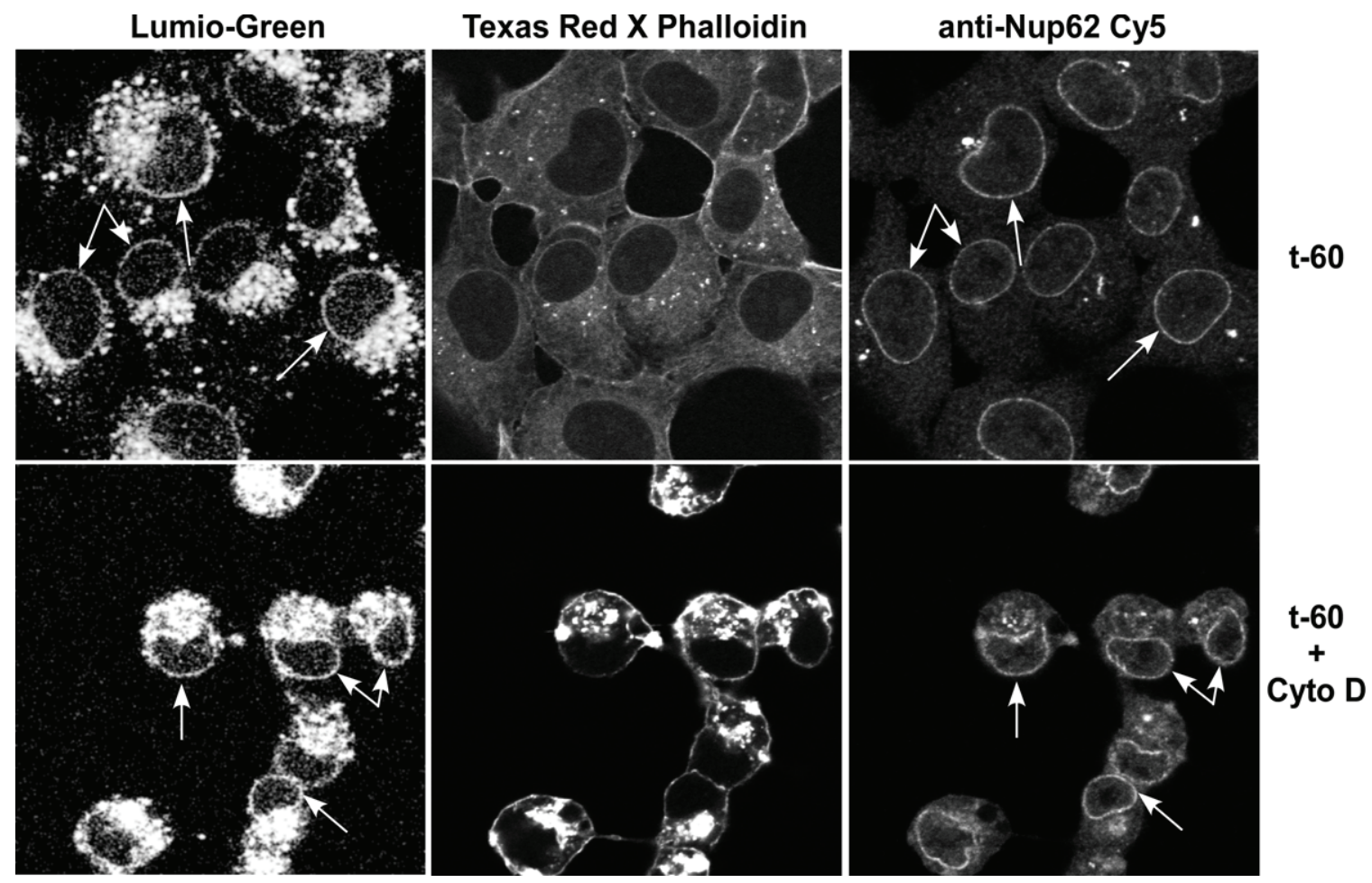

Figure 2-8. Distribution of M-Lumio-Green in the presence of cytochalasin D. (Top panels) Representative images of fixed cells from t-60 of a synchronized fusion assay showing distribution of M-Lumio-Green, actin, and Nup62. (Bottom panels) The same experiment and labeling as above except the cells were treated with cytochalasin D for 30 minutes before $\mathrm{NH}_{4} \mathrm{Cl}$ washout and cytochalasin $\mathrm{D}$ remained in the post washout media until cells were fixed 60 minutes later. Arrows indicate nuclear envelope localization. For all the images in this figure signal levels were enhanced using Canvas 11 software to maximize the Lumio-Green signal after conversion to grayscale (A and B). 


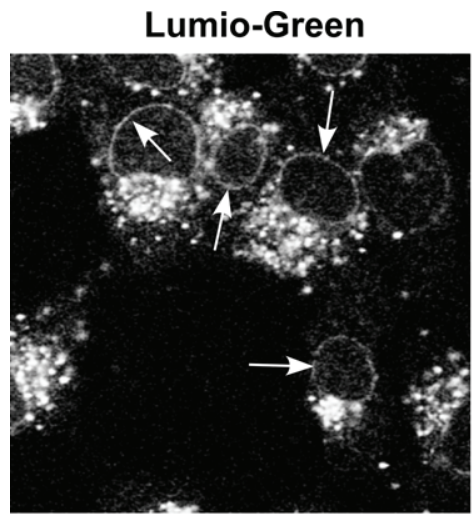
anti-tubulin Rhodamine

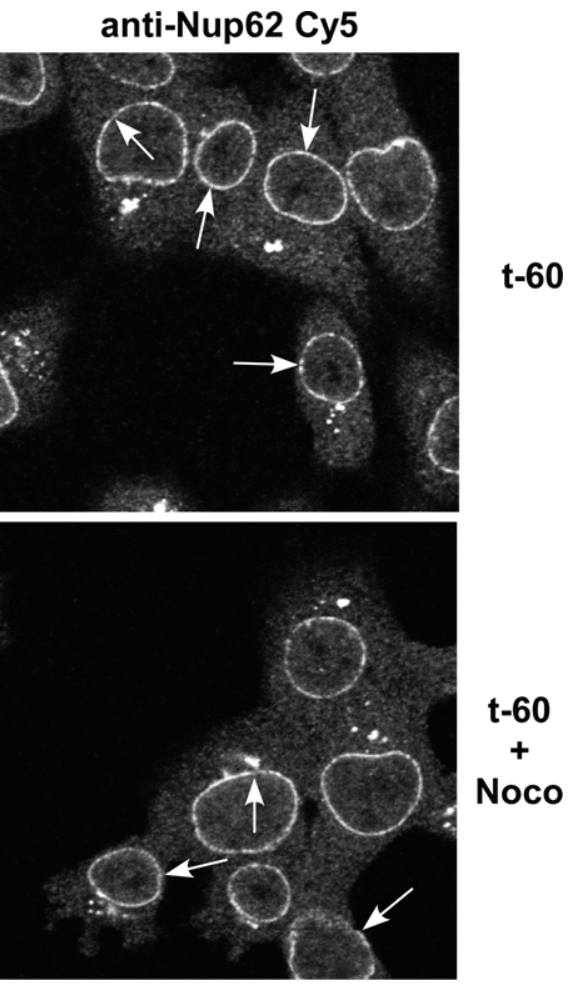

Figure 2-9. Distribution of M-Lumio-Green in the presence of nocodazole. (Top panels) Representative images of fixed cells from $\mathrm{t}-60$ of a synchronized fusion assay showing M-Lumio-Green, tubulin, and Nup62. (Bottom panels) The same experiment and labeling as in the top panels except the cells were treated with nocodazole to depolymerize microtubules for 30 minutes before $\mathrm{NH}_{4} \mathrm{Cl}$ washout and cells were maintained in the presence of nocodazole until the cells were fixed at $\mathrm{t}-60$. Arrows indicate nuclear envelope localization. For all the images in this figure signal levels were enhanced using Canvas 11 software to maximize the Lumio-Green signal after conversion to grayscale (A and B). 
A

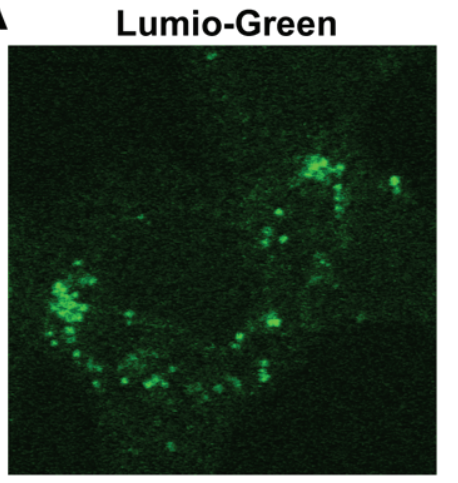

B

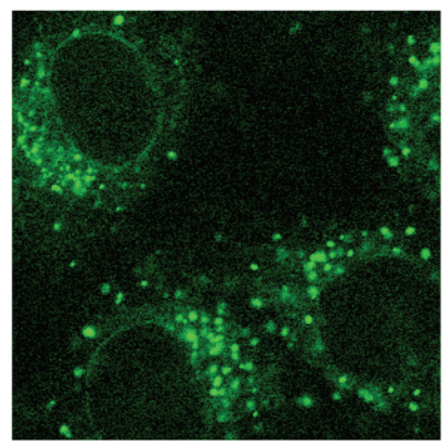

C

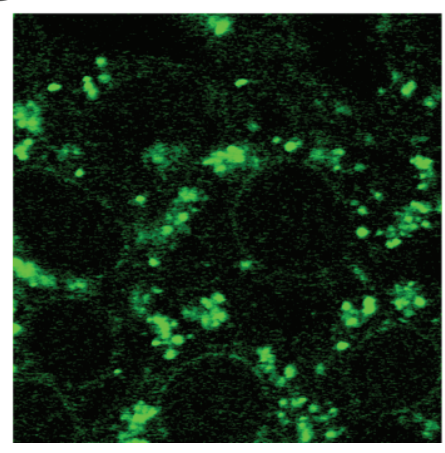

anti-N Rhodamine
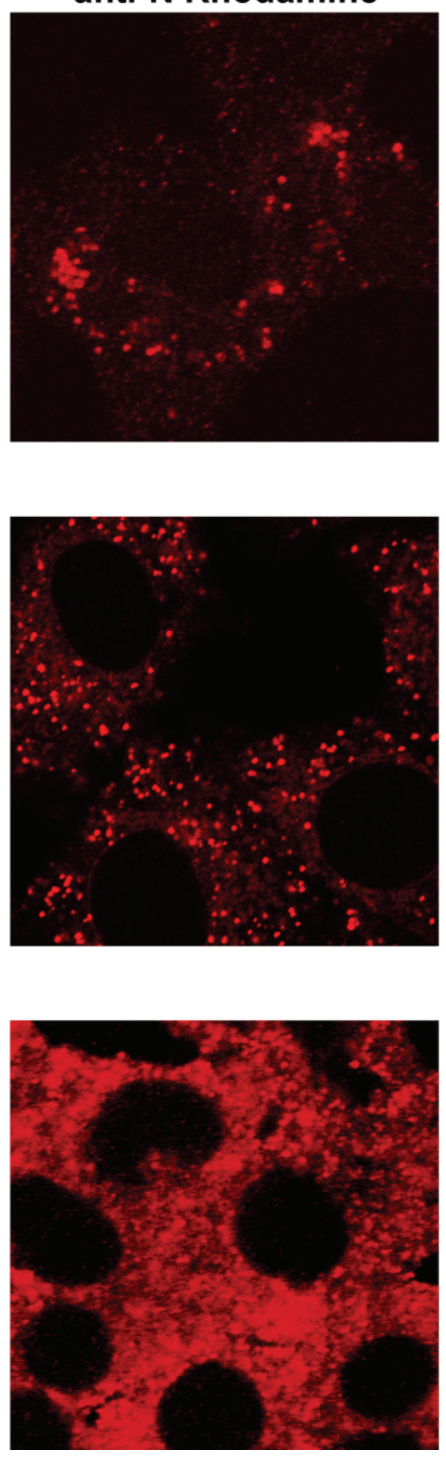

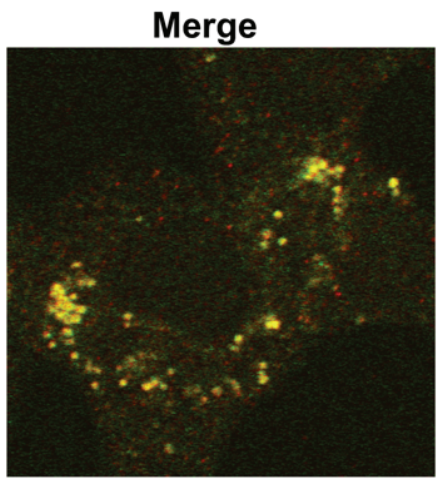

$\mathrm{M} / \mathrm{N}$

$92.8 \%$

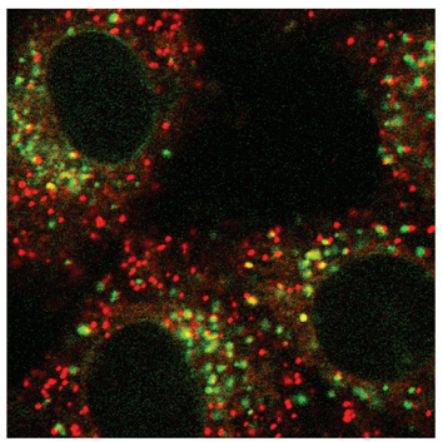

$31.3 \%$

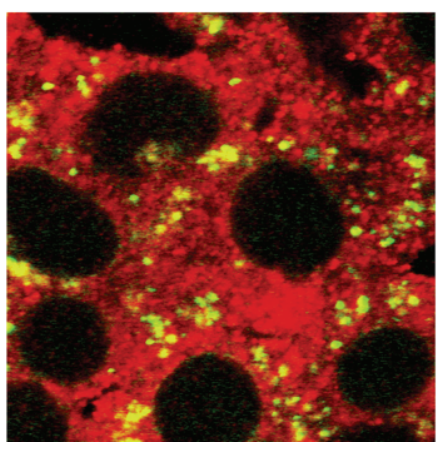

Figure 2-10. Release of RNPs from M-Lumio endosomes and productive infection in the presence of nocodazole.

Representative confocal images of cells inoculated with rVSV-M Lumio-Green (MOI 100) at t-0 (A) and t-120 (B and C), post $\mathrm{NH}_{4} \mathrm{Cl}$ washout, in the presence of cycloheximide and nocodazole (B) or just nocodazole $(\mathrm{C})$, and stained for VSV N protein using $\mathrm{N} \mathrm{mAb}$ conjugated to rhodamine. Colocalization of $\mathrm{M}$ and $\mathrm{N}$ was measured post fusion using the colocalization function in the LSM software version 3.2 and the percent colocalization of $(\mathrm{M} / \mathrm{N})$ is indicated for $\mathrm{t}-0$ (A) and t-120 (B). 
tagged with GFP and studied, there have been no reports of fluorescently tagged $\mathrm{M}$ proteins within virions for the study of M's fate after entry. Here we report the development and characterization of rVSVs with fluorescently tagged M using GFP (rVSV-M-MFC) and Lumio ${ }^{\mathrm{TM}}$ technology (rVSV-M-Lumio-Green). rVSV-M-LumioGreen was the best of these two virions for entry studies where the fate of the M-LumioGreen protein could be followed without the need for antibodies and due to epitope restriction in previous studies could be seen for the first time at the nuclear envelope after entry (Fig. 2-6, bottom panel arrows).

Previously, the M protein was fused to GFP and GST to study the nucleocytoplasmic transport, nuclear localization signal $(69,168,204)$, and mitochondrial localization (123) of the M protein. Except for the mitochondrial localization study (123), the majority of these fusions were at the N-terminus. However, the effect of these $\mathrm{M}$ fusions on assembly of VSV virions was not examined. In these studies the $\mathrm{N}$ and $\mathrm{C}$-terminal fusions did not affect the $\mathrm{M}$ activity studied $(69,123,168)$. However, the $\mathrm{N}$-terminus of the $\mathrm{M}$ protein is important in the assembly of virions as this portion of the protein contains the membrane association region $(30,31,120,217)$ and the PY motif $(80,91)$. In particular, membrane associated $M$ has been shown to be important for binding RNPs to the membrane and is thought to act as a bridge between the membrane and RNP of virions $(30,31)$. In addition, the PY motif at the N-terminus of $\mathrm{M}$ is important for budding activity and therefore budding of virions from the host cell $(80,91)$. With this in mind we fused GFP to the C-terminus (Fig. 2-1A, MFC).

Surprisingly, we found that large C-terminal extensions, such as GST and GFP, can affect M protein's budding activity (Fig. 2-2B lanes 3 and 4) although there was no apparent effect on $\mathrm{M}$ distribution to the plasma membrane (Fig. 2-2A). To our knowledge, this is the first example of a fusion at the C-terminus of $\mathrm{M}$ affecting M's budding activity, whereas only N-terminal mutations have been previously shown to effect this activity (91). Attempts to recover virions expressing MFC proteins (-II, -III, -IV, and M-GST as well) as the sole $M$ contributing to assembly were unsuccessful and we attributed this failure to the inability of these proteins cause budding. We were able to recover rVSV expressing both Mwt and MFCs and when the location of MFC within rVSV-M-MFC virions was examined in Figure 2-2D, it was found to be associated mainly with the RNP pellet (lane 9) versus the solubilized membrane fraction (lanes 10). This result showed that MFC, despite the large C-terminal extension, associated with RNPs and was almost excluded from the membrane of virions, which was surprising since MFC was clearly at the plasma membrane of rVSV-M-MFC infected cells (Fig. 2-2E, arrows). Although the majority of MFC within the virions was found with RNPs, it was only present at $5 \%$ of the total $\mathrm{M}$ protein population. Assuming that each virus particle has approximately 1,800 molecules of M protein (199), rVSV-M-MFC virions would average around 90 molecules of MFC. Based on a previous report, this amount of GFP within the virions may not be enough to visualize by microscopy since it was shown that 120 molecules of eGFP were sufficient for detection of individual rotavirus particles (28) whereas PeGFP virions (VSV-PeGFP) containing almost half of the 450 approximate copies of $\mathrm{P}$ per nucleocapsid could be visualized under high magnification, live-cell microscopy (42). The discrepancy between rVSV-M-MFC ( $\sim 90$ MFC copies) and VSV PeGFP $(\sim 225$ PeGFP copies) virions could also be due to the inability of the GFP fused to $M$ to 
undergo the proper conformational change to fluoresce. Also, there could be one population of virions with high MFC content and others with hardly any MFC which could lead to our inability to correlate plaque forming units (PFU) and percent MFC with the expected fluorescence at laser excitation of $488 \mathrm{~nm}$, especially when one considers the particle to PFU ratio of 1 to 200 (145) or 1 to 1,000 for VSV (unpublished lab data comparing genomic RNA to PFU).

We were able to recover rVSV with fluorescent $\mathrm{M}$ using Lumio $^{\mathrm{TM}}$ technology which utilizes a tetracysteine (-CCRECC-) motif that binds to membrane permeable Lumio-fluorophores that fluoresce when bound to this motif $(2,70)$. We fused the Lumio $^{\mathrm{TM}}$ tag to the $\mathrm{N}$ and $\mathrm{C}$ terminus of $\mathrm{M}$ and observed that the distribution of Lumio$\mathrm{M}$ and M-Lumio was similar to Mwt and unlike the larger $\mathrm{C}$-terminal extension of MFC, M-Lumio with its smaller extension was able to retain a budding activity comparable to Mwt (Fig. 2-3B lane 6), whereas Lumio-M was reduced in its budding activity (Fig. 2-3B lane 4). Lumio-M or M-Lumio was able to support VSV assembly as the sole M protein expressed from rVSV-M-Lumio genomes. Characterization of the growth kinetics of the recombinants showed that rVSV-M-Lumio was the best virus to use of the two tagged versions (Fig. 2-3C) and the protein to PFU ratio (Fig. 2-4B, left-hand gel) showed that this construct was able to grow and incorporate other viral proteins to similar levels as wild-type virus. The N-terminal tag results supported our original apprehension of fusing a tag at that terminus. Next, we showed that M-Lumio could be labeled with Lumio ${ }^{\mathrm{TM}}$ Green (M-Lumio-Green) during infection and unlike the entry study involving the labeling of HIV-1 integrase protein within virions with Lumio ${ }^{\mathrm{TM}}$ reagent in vitro (5), we could only label rVSV-M-Lumio virions within cells. Even though Lumio ${ }^{\mathrm{TM}}$ Green is membrane permeable, rVSV-M-Lumio virions were not able to be labeled in vitro and we hypothesize that this may have to do with the compact nature of the M-Lumio within the virion where the Lumio ${ }^{\mathrm{TM}}$ tag is not accessible or cannot flex in order to bind to the fluorophore.

In the synchronized entry assays, the bulk of M-Lumio-Green at 20 minutes post warm-up was associated with perinuclear endosomes and a smaller proportion at the nuclear envelope. This pattern was interesting as it was shown previously that $\sim 15 \%$ of input $\mathrm{M}$ could be found soluble within the cytosol after entry (174) and may account for our observation that the bulk of input $\mathrm{M}$ is not in the cytosol. In the synchronized fusion assays, we were able to show that M-Lumio-Green localized to the nuclear envelope after fusion (Fig. 2-6, bottom panels). We believe the localization to the nuclear envelope is indicative of M-Lumio-Green associating with nuclear pore complexes (NPCs), as the localization is in the same area as Nup62 as shown by the blue staining in the merged image of figure 2-6. We did not expect to see clear colocalization of the proteins since $\mathrm{M}$ associates with RaeI and Nup98 at the NPCs (53) and not directly with Nup62. The nuclear envelope association was similar to that of GFP-M association with NPCs as shown in a study of M binding to NPCs (168). This same study reported that M is not labeled by the monoclonal antibody (23H12) for M (Fig. 6, t-60, arrows, anti-MRhodamine), which recognizes the motif that binds to RaeI, and can prevent its binding and localization to the NPCs $(53,168)$. Because we did not require antibody to visualize M-Lumio-Green we were able to observe released $M$ that was distributed to the nuclear 
envelope and to our knowledge this is the first reported observation of this type of distribution after entry. We propose that upon VSV entry into host cells, released M protein associates at the NPCs to block nucleocytoplasmic transport (84), giving the virus an advantage over the host cell by blocking host cell mRNA transport (84) and thus the innate immune response (193) before viral protein synthesis has begun.

To address what the M-Lumio-Green associated perinuclear bodies were, we looked at markers for the endocytic pathway that corresponded to lysosomes, late endosomes/lysosomes, and early/recycling endosomes. A small proportion of M-LumioGreen colocalized with LAMP-1 and Mannose-6-Phosphate receptor (Fig. 2-7A and B), however the bulk of colocalization was with transferrin-TR (Fig. 2-7C). At higher magnification, we showed the M-Lumio-Green distribution within the endosome was asymmetric (Fig. 2-7D, arrows). In contrast, we observed a uniform distribution for noninfectious internalized $\Delta \mathrm{G}-\mathrm{rVSV}$-M-Lumio-Green virions suggesting the asymmetric distribution seen after entry of rVSV-M-Lumio-Green was G-dependent and could be the result of one of two conditions. A model for an increase in $\mathrm{G}$ protein binding with endosomal membranes at low $\mathrm{pH}$ has previously been proposed (59) and could explain the rVSV-M-Lumio-Green asymmetric distribution as virions that have not fused could still be bound to the inner leaflet of the endosomal membrane whereas $\Delta \mathrm{G}$ virions would not be able to bind in this manner resulting in the difference between the two distributions. In the other condition, the increased binding affinity of $\mathrm{G}$ at low $\mathrm{pH}$ could result in fusion of virions to one side of the endosome and the place where fusion occurs could be marked by the membrane bound $\mathrm{M}$ population that is now on the outside of the endosomal membrane after RNPs are released and this could not be seen as $\Delta \mathrm{G}$ virions lack the ability to fuse with the endosomal membrane. Here we hypothesize that the viral envelope associated M-Lumio-Green is on the cytosolic side of the endosomal membrane of early endosomes, after fusion, at the site of fusion.

While there is a need for microtubules to traffic vesicles within the endocytic pathway (74), it has been shown that VSV has the ability to fuse with early endosomes and release its genome prior to Rab7 dependent trafficking $(54,189)$. The distribution observed after fusion (Figs. 2-8 and 9) and the $\mathrm{M}$ and $\mathrm{N}$ separation (Fig. 2-10B) in the presence of cytoskeletal inhibitors seem to support the hypothesis that fusion and release from early endosomes is the main entry pathway. The change in $\mathrm{N}$ staining (Fig. 2-10A and B) at t-120 in the presence of nocodazole and cycloheximide could be the result of two conditions; (i) protein synthesis was not completely blocked or (ii) the uncoating of genomes has increased the amount of $\mathrm{N}$ protein that is available for staining, since the scanning parameters (detector and gain settings) and amount of antibody for each condition were kept the same. We also observed some LAMP-1 and M-6-P-R colocalization with virus in Figures 2-7A and B which showed VSV can reach later points in the endocytic pathway such as the endosomal carrier vesicles/multivesicular bodies further along the pathway where it has been reported to undergo a back fusion event for entry (117).

The ability of M-Lumio-Green to localize to the nuclear envelope without fully polymerized actin (Fig. 2-8, arrows bottom panel) or tubulin (Fig. 2-9, arrows bottom 
panel) was unexpected since the localization seen in the synchronized entry assays occurred within 20 minutes of post warm-up (Fig. 2-5, arrows bottom panel) which suggested trafficking would be involved, along with the fact that $M$ has nuclear localization signals (69). We conclude that after uncoating, M-Lumio-Green distributes to the nuclear envelope without the full cytoskeletal network that is known to contribute to transport within cells (183) and therefore may get there by diffusion, but we also have not directly ruled out delivery of $\mathrm{M}$ to the nuclear envelope by vesicular trafficking. Also it has been shown that RaeI binds to microtubules in mitotic spindle complexes $(17,215)$ and M may bind this protein in the cytoplasm and enter the NPCs in this manner.

Here we considered delivery of M-Lumio-Green to the nuclear envelope (Fig. 29, bottom panel arrows), separation of $\mathrm{M}$ and $\mathrm{N}$ (Fig. 2-10B), and productive infection (Fig. 2-10C) after microtubule dissociation as clear indicators that uncoating occurs at early endosomes along the endocytic pathway. The distribution that we observed was different from the completely cytosolic and nuclear distribution of $\mathrm{M}$ we expected after uncoating (174). This difference could be attributed to the M labeling technique, lower MOIs, and the confocal microscopy used for this study. Instead of visualizing uncoating as $\mathrm{M}$ being distributed throughout the cytosol as expected, we have shown that this process is visualized by asymmetric association of $\mathrm{M}$ with endosomes possibly at sites of fusion and delivery of a fraction of M to the nuclear envelope. Use of rVSV with MLumio-Green and other viral proteins fused to red and yellow fluorescent proteins would allow for a tri-fluorescent virus to study entry in greater detail giving us further insight into the mechanisms of VSV uncoating. 


\section{Chapter 3: Low pH Enhancement of Matrix Uncoating}

\section{Introduction}

Vesicular stomatitis virus (VSV), a prototype enveloped, nonsegmented, negative-strand RNA virus in the family Rhabdoviridae, is composed of an $11 \mathrm{~Kb}$ genome that encodes five genes (3'-N-P-M-G-L-5'). These genes are the nucleocapsid protein $(\mathrm{N})$, the phosphoprotein $(\mathrm{P})$, the matrix protein $(\mathrm{M})$, the glycoprotein $(\mathrm{G})$, and the large RNA dependent RNA polymerase protein $(\mathrm{L})$. The virion's structure is composed of a helical nucleocapsid, containing L-P-N-RNA-M, surrounded by a membrane derived from an infected host cell membrane with transmembrane, trimeric $\mathrm{G}$ proteins protruding from the surface. These bullet-shaped VSV particles are $180 \mathrm{~nm}$ long and $75 \mathrm{~nm}$ wide with around 400 trimeric $G$ protein spikes on the exterior of the viral membrane and about 1,800 molecules of $\mathrm{M}$ protein between the nucleocapsid core and the viral membrane (199). The RNA genome is encapsidated by N protein with around 1,200 molecules of this protein tightly complexed to the RNA like beads along a string (199). The RNA-dependent RNA polymerase, consisting of the L and P proteins, is associated with this ribonucleoprotein complex and these proteins are present at 50 and 500 molecules per virion, respectively (199). This L-P-N-RNA complex makes up the ribonucleoprotein particle (RNP) which is coiled into a tightly packed helix of around 35 turns inside the viral membrane. The M protein condenses the RNP into this tightly packed helix, which, when condensed as described, is referred to as the skeleton (153, 154). A population of membrane bound $M$ anchors the skeletons to the viral membrane $(31,151)$.

When VSV enters the host cell through the clathrin-mediated endocytic pathway (139), it uses the decreasing $\mathrm{pH}$ of endocytic organelles as a trigger for fusion in order to penetrate the cell as is the case for influenza virus (207). To enter the cell VSV virus attaches to the surface and is endocytosed by coated vesicles and transported through the endosomal pathway to early or late endosomes. In early endosomes, $\mathrm{pH}$-dependent fusion of the endosomal and viral membrane occurs and subsequent virus uncoating and release of the genome in the form of the RNP $(114,136)$. The G protein of VSV is directly responsible for entry steps attachment and fusion $(18,82,134,161,198,211)$ where fusion results in the release of the RNP core into the cytoplasm where VSV replication occurs (139). Either directly after or concomitant with membrane fusion, $\mathrm{M}$ protein dissociates from the RNP core which results in the decondensation of the skeleton $(153,154)$ and completes uncoating (174). After uncoating, the decondensed RNP serves as a template for transcription of viral mRNAs by the packaged RNA-dependent RNA polymerase.

Most enveloped virus particles entering a cell have highly condensed genomes protected by proteins. Before these particles can replicate in a host cell, after fusion and entry, uncoating is required and this is described as the dissociation of some proteins that keep the genome in a condensed or a biologically inactive state. The interesting aspect of uncoating is that this process is the opposite of what occurs to package the virus for 
release. Here lies the dilemma; how does the same protein of a virus accomplish two distinct processes in the same intracellular environment. Viral uncoating, in the case of $\mathrm{VSV}$, is regarded as the dissociation of M from RNPs and is an essential step in entry (130). Without the release of M protein transcription of viral mRNAs (productive infection) cannot proceed since it has been shown that $M$ protein inhibits transcription $(32,44,212)$. This essential step is also shared by influenza virus, another negativestrand RNA virus, which requires removal of its matrix protein (M1) for a productive infection $(102,137)$. While in endosomes the M2 protein of influenza virus acts as an ion channel $(110,169)$ that allows protons across the viral envelope $(81)$. Acidification of the virus interior decreases the interaction between M1 and nucleocapsids allowing for their dissociation before entry of the nucleocapsids into the cytoplasm (222). VSV lacks a similar proton channel, though previous studies have found that spike proteins of enveloped viruses such as VSV, Sindbis virus, and Semliki Forest virus and Ebola virus GP2 can increase membrane permeability $(76,101,112)$. Though not studied in the context of virus it has been suggested that the haemaglutinin (HA) protein of Influenza at the $\mathrm{pH}$ required for fusion can also cause cell permeability and proton influx when found on the cell membrane (182). As with influenza virus M1, VSV M protein is found within the cytoplasm of the host cell in soluble form separated from nucleocapsids (174). However, the regulatory elements involved in the dissociation of M protein from RNPs are still not understood. In vitro studies using biophysical measurements at physiological ionic strength $(60-90 \mathrm{mM} \mathrm{NaCl})$ showed that the association of $\mathrm{M}$ with the RNP had the characteristics of a dynamic reversible equilibrium (130). This suggested that $\mathrm{M}$ protein will dissociate from skeletons in the cytoplasm of newly infected cells, and later in infection the concentration of $\mathrm{M}$ is high enough for equilibrium to be reached with regards to cytoplasmic and skeleton associated $\mathrm{M}$ (130). These in vitro observations could explain why dissociation and association of $\mathrm{M}$ can occur under the same ionic conditions, however little is known about the contribution of host factors in dissociation and the distribution of $\mathrm{M}$ protein during uncoating.

To study the release of $\mathrm{M}$ protein during uncoating, we developed and characterized a recombinant VSV (rVSV, (rVSV-M-Lumio-Green)) encoding an M protein that could be fluorescently tagged and gave us the ability to follow the fate of fluorescent $\mathrm{M}$ during entry of virions (Chapter 2). Here, we report that there is a transient loss of fluorescence intensity of rVSV-M-Lumio-Green virions at early times after the initiation of endocytosis in a synchronized entry assay. In vitro, rVSV-M-Lumio-Green particles had strong fluorescence when excited at a wavelength of $508 \mathrm{~nm}$ at $\mathrm{pH} 7$, but there was a dramatic loss in fluorescence when the $\mathrm{pH}$ was lowered. Based on the $\mathrm{pH}$ dependent fluorescence properties of Lumio ${ }^{\mathrm{TM}}$ Green, this observation suggested that the interior of the virion was becoming acidified. We found that the effect of $\mathrm{pH}$ on the $\mathrm{M}$ Lumio-Green fluorescence within intact virus was G-dependent and further biochemical analysis indicated that low $\mathrm{pH}$ resulted in an enhancement of $\mathrm{M}$ dissociation from partially permeabilized but intact virions. We propose a model for the initial uncoating events of VSV based on our observations and past viral spike protein membrane disruption studies $(76,101,112,182)$. 


\section{Materials and Methods}

Viruses. rVSV-wt was used in biochemical analysis of ${ }^{35}$ S-Methionine labeled virions. rVSV-M-Lumio-Green (Chapter 2) was used in live-cell synchronized entry assays (in BHK-21 cells at MOI 50) and in the fluorometric assays. $\Delta$ G-rVSV-MLumio-Green pseudotyped with either G or G-stem (GS) with a N-terminal haemaglutinin (HA) tag ( $\mathrm{GS}^{\mathrm{HA}}$ ) complemented $\Delta \mathrm{G}$-rVSV-M-Lumio-Green were also used in the fluorometric assays. $\Delta$ G-rVSV-M-Lumio-Green were complemented with G or $\mathrm{GS}^{\mathrm{HA}}$ by methods used in previous studies (93) with some modifications. Briefly, BHK cells at $\sim 80 \%$ confluency were transfected with $2 \mu \mathrm{g}$ of $\mathrm{pC}-\mathrm{G}$ or $-\mathrm{GS}^{\mathrm{HA}}$ plasmid and infected with G-complemented $\Delta$ G-rVSV-M-Lumio-Green at MOI 10 at 18 hours post transfection. The Lumio-tagged viruses were labeled as done previously (Chapter 2) using 200nM Lumio ${ }^{\mathrm{TM}}$ Green in Opti-MEM I at 4 hours post infection. Eighteen hpi the viral supernatants were collected and ultracentrifuged over a $20 \%$ sucrose cushion at $38,000 \mathrm{rpm}$ in an SW41 swinging bucket rotor (Beckman) to pellet the virions. The supernatant and sucrose cushion were then removed by aspiration and the viral pellet was resuspended on ice in $10 \%$ sucrose TN solution $(10 \mathrm{mM}$ Tris- $\mathrm{HCl}$ [pH 8.0], $150 \mathrm{mM}$ $\mathrm{NaCl}$ ). Residual unbound Lumio $^{\mathrm{TM}}$ Green reagent was removed from the virus preparation by passing $100 \mu \mathrm{l}$ aliquots through Sephadex G-50 (Fine) columns (Roche) by centrifugation according to the manufacturer's instructions. The void volume was then analyzed for the fluorescence intensity using a fluorometer (Perkin Elmer LS 50 B) and protein composition was analyzed by Coomassie stained SDS-PAGE in which equivalent fluorescence was loaded. Viral preparations were used immediately resulted in disruption of the viral envelopes.

Live-cell synchronized entry assay. To examine entry of labeled virus into live cells, $\Delta \mathrm{G}$ or rVSV-M-Lumio-Green (MOI 50) together with $50 \mu \mathrm{g}$ of transferrin-TR were added to BHK-21 cells in $35 \mathrm{~mm}$ glass bottomed culture dishes (MatTek Corporation) at $90 \%$ confluency after cells had been washed twice with $2 \mathrm{ml}$ of ice-cold Opti-MEM I. After the ice-cold wash, the plates were placed at $4^{\circ} \mathrm{C}$ for 10 minutes and (as done previously Chapter 2). The virus and transferrin-TR were adsorbed for 90 minutes in 100 $\mu \mathrm{l}$ ice-cold Opti-MEM I with rocking every 15 minutes at $4^{\circ} \mathrm{C}$. The cells were covered to prevent light photobleaching. Five plates of cells were used per experiment, each corresponding to a 5 minute time point post entry. After the 90 minute adsorption, $37^{\circ} \mathrm{C}$ media was added to initiate entry. The binding of rVSV-M-Lumio-Green and transferrinTR at the non-entry time point $(\mathrm{t}-0)$ was observed by laser scanning confocal microscopy (Zeiss LSM 510) following addition of $2 \mathrm{ml}$ of ice-cold Opti-MEM I. Separate plates were used to examine entry after addition of $37^{\circ} \mathrm{C}$ media $5,10,15$, and 20 minutes (t-5, t$10, \mathrm{t}-15, \mathrm{t}-20)$ by confocal microscopy. Pixel intensity of rVSV-M-Lumio-Green fluorescence was analyzed using the Profile function of LSM software (version 3.2).within transferrin-TR containing endosomes and then plotted as mean pixel intensity as a function of time. GraphPad software was used to calculate the P values by the twotailed t-test for the statistical analysis of the mean pixel intensity. 
Fluorometric assay. To measure the fluorescence intensity of rVSV-M-LumioGreen virions in vitro, we placed $1 \mu \mathrm{g}$ of the virus in HMN (HEPES 10mM, MES 10mM, $\mathrm{NaCl} 150 \mathrm{mM}, \mathrm{pH} 7$ ) buffer and measured the fluorescence signal over 10 seconds at 1 second intervals for each measurement with a Perkin Elmer LS 50B. To determine the effect of $\mathrm{pH}$ on fluorescence intensity of, we recorded the fluorescence signal at $\mathrm{pH} 7$ and added $1 \mathrm{M} \mathrm{NaOH}$ to raise the $\mathrm{pH}$ to 7.5 and then 8 taking readings at each point. We then dropped the $\mathrm{pH}$ by adding $1 \mathrm{M} \mathrm{HCl}$ to reach a $\mathrm{pH}$ of $6.5,6,5.5$, or 5 with fluorescence signal recorded for each $\mathrm{pH}$. These readings were then quantified as a percentage of fluorescence intensity loss from the $\mathrm{pH} 7$ signal.

To determine if $\mathrm{G}$ was important for the decrease in fluorescence intensity signal of rVSV-M-Lumio-Green virions, we applied the same protocol for the $\mathrm{pH}$-dependent fluorescence intensity measurements of $\Delta$ G-rVSV-M-Lumio-Green and GS ${ }^{\mathrm{HA}}$ complemented $\Delta$ G-rVSV-M-Lumio-Green virions. These virions were normalized to the same fluorescence signal for each experiment (since these are not infectious) and were based on the $\Delta$ G-rVSV-M-Lumio-Green signal since this virus produces the lowest amount of virus. Virions were then analyzed by SDS-PAGE based on their fluorescence signal. To determine if the viral envelope was a barrier to the $\mathrm{pH}$ effect on M-LumioGreen signal, virions were solubilized HMN buffered to $\mathrm{pH} 5.5$ with $0.1 \%$ Triton $\mathrm{X}-100$ $(\mathrm{w} / \mathrm{v})$ after the initial readings at $\mathrm{pH} 5.5$, and the percent fluorescence loss was measured from $\mathrm{pH} 7$ readings.

${ }^{35}$ S-methionine labeling of rVSV-wt. BHK-21 cells were infected with rVSVwt at MOI 5. At 4 hours post infection the cells were starved of methionine for 30 minutes and then ${ }^{35} \mathrm{~S}$-methionine in methionine-free DMEM $(50 \mu \mathrm{Ci} / \mathrm{ml})$ was added to the cells. Fourteen hours post infection the virus was isolated from the supernatant by ultracentrifugation (27K rpm, Beckman SW-28) over a 20\% sucrose TN cushion for 1.5 hours. The amount of protein in the viral preparation was determined using a BCA protein assay (Pierce) following the manufacturer's instructions.

Lysolecithin treatment of rVSV-wt. To test the effect of on internal VSV proteins, we applied a method of gently "poking holes" in the viral envelope using the mild detergent lysolecithin that has been used to permeabilize cells in other studies (6, 124). To determine the appropriate concentration to use, we treated $1 \mu \mathrm{g}$ of ${ }^{35} \mathrm{~S}$ methionine labeled rVSV-wt with varying concentrations of lysolecithin ranging from 0 , $3.9,7.8,15.6,31.2$, and $62.4 \mu \mathrm{g} / \mathrm{ml}$ at $\mathrm{pH} 5.5$ for 10 minutes on ice. The suspension was ultracentrifuged for 35 minutes $(45 \mathrm{~K}$ rpm, Sorvall AH-650) over a $20 \%$ sucrose TN (10 $\mathrm{mM}$ Tris- $\mathrm{HCl} \mathrm{pH} \mathrm{8,} \mathrm{NaCl} 150 \mathrm{mM}$ ) cushion to separate the proteins in the supernatant from the virus that pelleted to the bottom of the tube. The supernatant was TCA precipitated, resuspended in reducing sample buffer, and loaded onto SDSpolyacrylamide gels and compared to the whole pellet by fluorography.

To compare protein in the supernatant of virions treated with lysolecithin at $\mathrm{pH} 7$ and $\mathrm{pH} 5.5$, we treated $1 \mu \mathrm{g}$ of ${ }^{35} \mathrm{~S}$-methionine labeled rVSV-wt with lysolecithin at a 
concentration of $3.9 \mu \mathrm{g} / \mathrm{ml}$ in $\mathrm{HMN}$ buffer at $\mathrm{pH} 7$ or $\mathrm{pH} 5.5$. We prepared the mixes as stated above and quantitated the amount of ${ }^{35} \mathrm{~S}$-methionine labeled $\mathrm{M}$ protein in the supernatant to the $\mathrm{N}$ protein in the pellet by phosphorimaging.

TEM of rVSV-wt treated with lysolecithin. To confirm virions were not disrupted by lysolecithin treatment $1 \mu \mathrm{g}$ of rVSV-wt was treated with lysolecithin (3.9 $\mu \mathrm{g} / \mathrm{ml}$ ) in HMN buffer at $\mathrm{pH} 7$ or $\mathrm{pH} 5.5$ and ultracentrifuged over a $20 \%$ sucrose cushion. The pellets were resuspended in $10 \%$ sucrose and placed on ice. To decrease the sucrose concentration, the virus preparations were then diluted $1: 10$ in $\mathrm{dH}_{2} \mathrm{O}$ and prepared for transmission electron microscopy (TEM) by adsorbing virus to a grid using the drop-to-drop method and negative staining with $2 \%$ phosphotungstic acid (PTA, pH 5.9).

\section{Results}

Loss of fluorescence intensity over time during rVSV entry. To follow the entry of $\Delta \mathrm{G}$ (bald) or rVSV-M-Lumio-Green (G containing) virions into live cells, we adsorbed virus (MOI 50) onto cells at $4^{\circ} \mathrm{C}$ to synchronize their entry as described in Chapter 2 for figure 2-5. We used transferrin conjugated to Texas Red (transferrin-TR) as an endosomal marker during endocytosis of the virions and the cells were observed using confocal microscopy every 5 minutes over a 20 minute span, post addition of media at $37^{\circ} \mathrm{C}$. Single images from parallel sets of plates were collected at identical detector and gain settings for each time point, and in which the threshold value was set to ensure no pixel saturation occurred. By keeping the settings the same, and below threshold, we quantified the average fluorescence intensity from multiple cells between time points. We observed that the fluorescence intensity of rVSV-M-Lumio-Green virions decreased over the time course of entry (Fig. 3-1A, left-hand graph). These data ( $\mathrm{n}=50$ per time point in triplicate) were compiled using the Profile function of the LSM 510 software (Zeiss version 3.2), which measures peak intensity and size of fluorescent signals for each scan. The size of the particles within endosomes from which the fluorescent intensity measurements were collected were no larger than 180nm, which is similar to the average length of a VSV virion (VSV's size is $\sim 180 \mathrm{~nm} \times 70 \mathrm{~nm}$ ). Only M-Lumio-Green signals that colocalized with transferrin-TR were used for quantification which ensured the fluorescence intensity measured was within an endosome. We observed the largest reduction in fluorescence intensity of rVSV-M-Lumio-Green particles at 15 minutes post addition of warm media, where the mean fluorescence intensity was $27 \%$ below that of t0 . This difference was statistically significant with a $P$ value less than 0.0001 using a paired t-test for the mean intensity of particles (Fig. 3-1A, right-hand graph). Also, when we compared the mean fluorescence intensity of $\Delta \mathrm{G}$ and rVSV-M-Lumio-Green particles at t-15 (Fig. 3-1B) the difference was found to be $27.3 \%$ which was also statistically significant $(\mathrm{p}<0.0001)$. 
Figure 3-1. $\Delta G$ and rVSV-M-Lumio-Green fluorescence intensity during entry. (A) From synchronized entry assay images in Chapter 2 (Fig. 2-5). rVSV-M-LumioGreen virions and transferrin-TR were bound to BHK-21 cells in cold media for 90 minutes, then the cells were washed and warm media was added to allow entry to occur. The fluorescence intensity of M-Lumio-Green particles, with transferrin-TR positive endosomes that measured $\sim 180 \mathrm{~nm}$ using the Zeiss LSM 50 profile software was read for 50 particles per time point in triplicate every 5 minutes for 20 minutes (left-hand graph). The difference in average fluorescence intensity at 0 minutes and 15 minutes post warm media addition was significant. (*, right-hand graph) $\mathrm{p}<0.0001$ using a paired t-test. (B) Comparison of the average fluorescence intensity of bald $\Delta \mathrm{G}$ and rVSV-M-LumioGreen virions 15 minutes post warm media addition. $\left(^{*}\right) \mathrm{p}<0.0001$ using a paired t-test. 
A
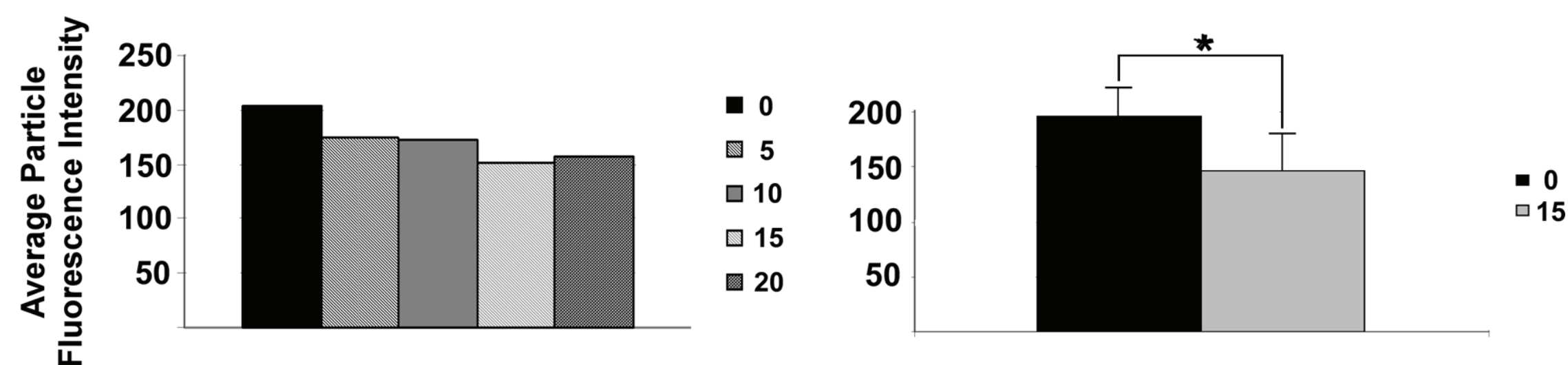

B

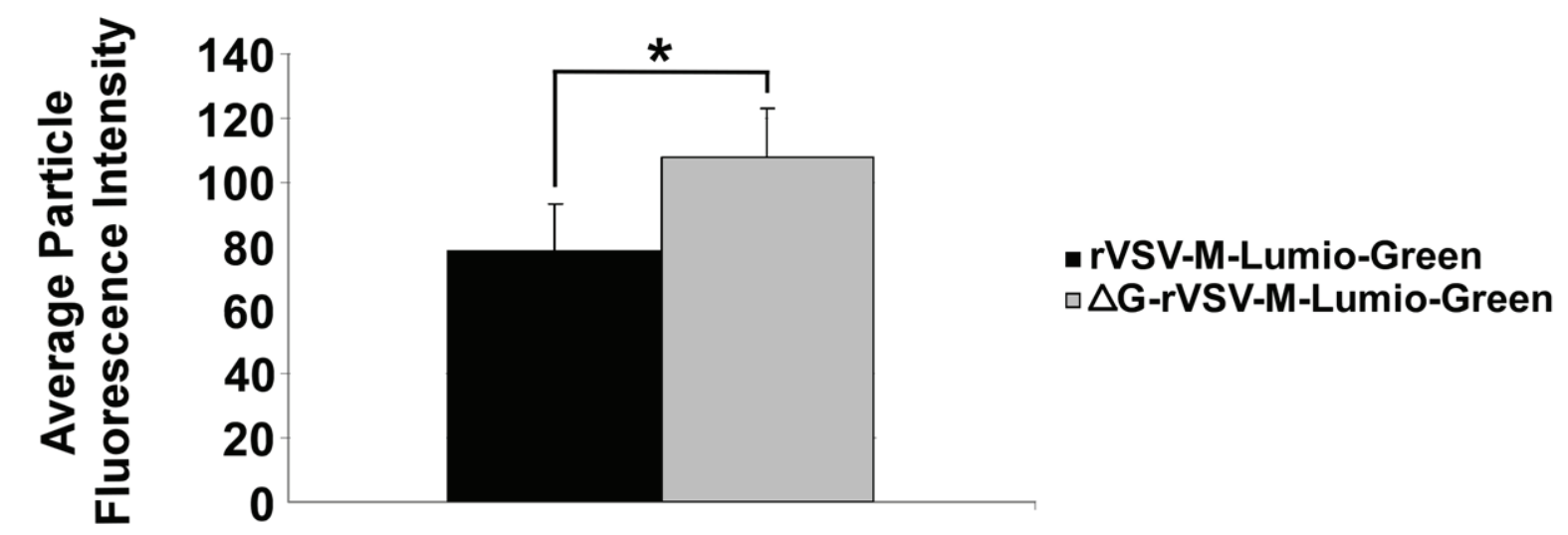




\section{Loss of fluorescence intensity of rVSV-M-Lumio-Green in the presence of}

low pH. In the live-cell entry assay we observed a difference in mean fluorescence intensity of particles when bound to the cell surface at $\mathrm{t}-0$ and at $\mathrm{t}-15$ where most of the endocytosed virus would be in endosomes at the appropriate $\mathrm{pH}$ for fusion of the viral envelope and endosomal membrane $(51,58,172)$ (Fig. 3-1A). This observation was interesting when one considers that the apparent $\mathrm{pKa}$ of Lumio $^{\mathrm{TM}}$ Green for the tetracysteine motif is $5.4(70)$. We reasoned that acid $\mathrm{pH}$ could be responsible for the decrease in fluorescence intensity during entry since VSV enters the cell through the clathrin-mediated endosomal pathway where the lumenal $\mathrm{pH}$ of endosomes drops as they move from early to late endosomes. To assess whether $\mathrm{pH}$ had an effect on the fluorescence of rVSV-M-Lumio-Green virions, we mimicked the drop in $\mathrm{pH}$ along the endosomal pathway by addition of $\mathrm{HCl}$ to a $\mathrm{HMN}$ (HEPES $10 \mathrm{mM}$, MES $10 \mathrm{mM}, \mathrm{NaCl}$ $120 \mathrm{mM}$ ) buffer and measured (in triplicate; standard deviations shown) the effect on rVSV-M-Lumio-Green fluorescence in vitro using a fluorometer. When excited at a wavelength of 508nm in HMN buffer at $\mathrm{pH} 7,1 \mu \mathrm{g}$ of rVSV-M-Lumio-Green virions gave an average fluorescence reading of $34.5(+/-4.7)$ at $528 \mathrm{~nm}$, when excited at a wavelength of $508 \mathrm{~nm}$. The fluorescence was similar at a $\mathrm{pH}$ of 7.5 and 8.0 ; however, we found that below $\mathrm{pH} 7$ the fluorescence of rVSV-M-Lumio-Green progressively decreased as the buffer became more acidic (Fig. 3-2A). These data confirmed that loss of Lumio ${ }^{\mathrm{TM}}$ Green fluorescence intensity was $\mathrm{pH}$-dependent. Interestingly, at a $\mathrm{pH}$ of 6, the $\mathrm{pH}$ at which G-mediated fusion occurs, we observed a similar loss of fluorescence intensity in vitro as was seen at t- 15 in cells ( $29 \%$ versus $27 \%$, respectively). We also observed that the curve for fluorescence intensity loss was similar to $\mathrm{pH}$ curves seen for VSV G protein-dependent fusion $(51,172)$. This similarity suggested that $\mathrm{G}$ somehow contributes to the loss in fluorescence, which we interpret as exposure of M-LumioGreen to acidic $\mathrm{pH}$ within the intact virions.

Fluorescence intensity loss of rVSV-M-Lumio-Green is G protein dependent. To determine if $\mathrm{G}$ was involved in the fluorescence intensity loss, we compared the responses of rVSV-M-Lumio-Green and $\Delta \mathrm{G}-\mathrm{rVSV}-\mathrm{M}$-Lumio-Green virions to low $\mathrm{pH}$. To determine if there was a difference in the incorporation of Lumio ${ }^{\mathrm{TM}}$ Green into $\Delta \mathrm{G}$ virus compared to virus containing $\mathrm{G}$ protein we evaluated the protein to fluorescence ratio by Coomassie stained SDS-PAGE gels. We observed that there was a similar amount of VSV proteins when normalized by fluorescence signal (Fig. 3-2B). We then tested whether $\Delta \mathrm{G}-\mathrm{rVSV}$-M-Lumio-Green virions would lose fluorescence in a manner like rVSV-M-Lumio-Green when exposed to low $\mathrm{pH}$. We measured the initial fluorescence of virions in $\mathrm{HMN}$ buffer at $\mathrm{pH} 7$ and added $\mathrm{HCl}$ to bring the $\mathrm{pH}$ to 5.5 (in triplicate) and measured fluorescence intensity again. The fluorescence signal of the $\mathrm{G}$ containing virus was reduced $57 \%$ while bald $\Delta \mathrm{G}$ virus lost only $8 \%$ (Fig. 3-2C, lefthand bars). To determine if removal the envelope of the $\Delta$ G-rVSV-M-Lumio-Green virions could result in the loss in fluorescence, we added detergent to the virion preps (both $\mathrm{G}$ containing and bald) to solubilize the envelopes and found that there was a loss in fluorescence intensity similar to rVSV-M-Lumio-Green virions (Fig. 3-2C, right-hand bars). 
A
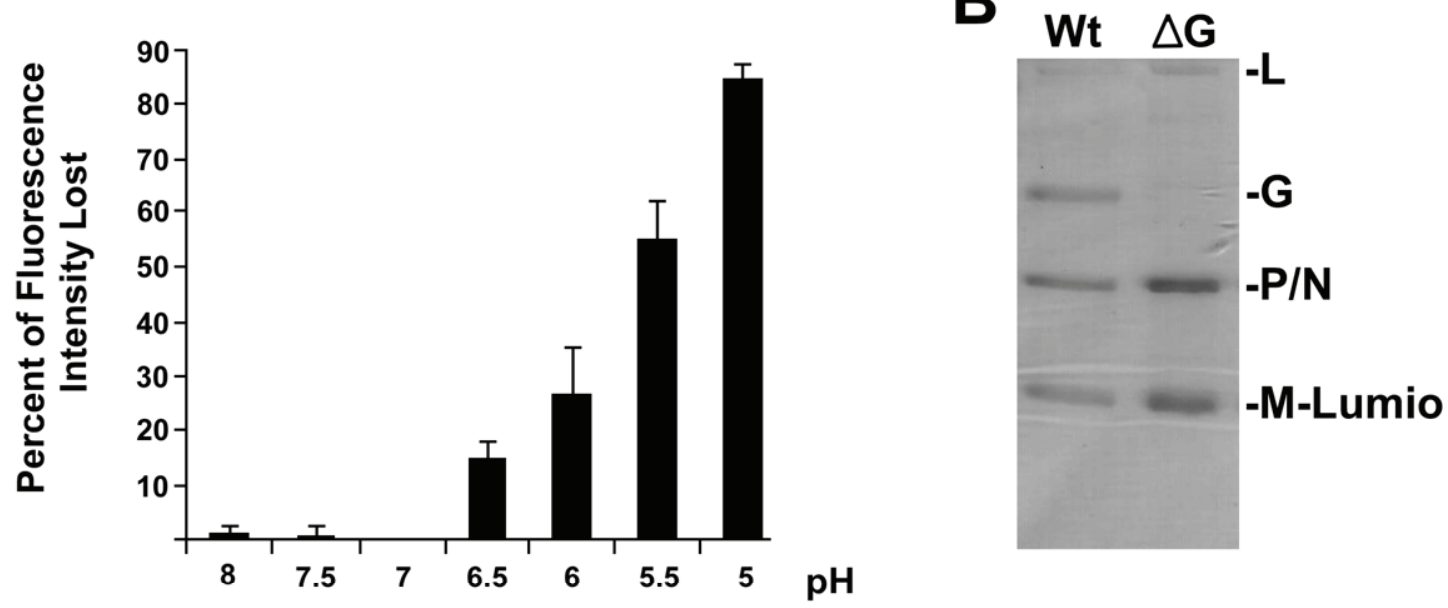

C

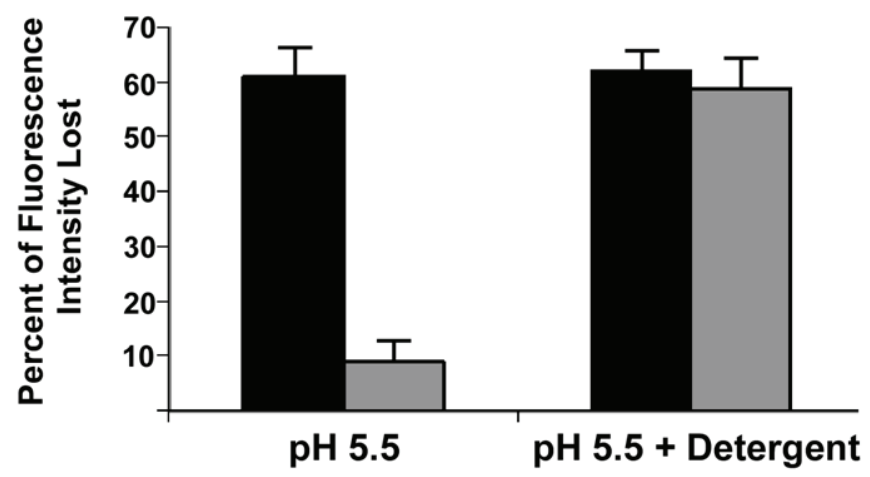

口-rVSV-M-Lumio

口- $\Delta$ G-rVSV-M-Lumio

Figure 3-2. pH- and G-dependence of fluorescence intensity loss.

(A) Percentage of fluorescence intensity loss over a $\mathrm{pH}$ range from 8 to 5.5 when compared to fluorescence at $\mathrm{pH}$ 7. rVSV-M-Lumio-Green virions were suspended in $\mathrm{HMN}$ buffered to the indicated $\mathrm{pH}$ and the fluorescence was read by a fluorometer in triplicate. Standard deviations are shown. (B) Representative Coomassie stained SDSPAGE of rVSV-M-Lumio-Green and $\triangle$ G-rVSV-M-Lumio-Green virions. The amount of virus loaded was based on the fluorescence signal and 35 fluorescence units were examined. (C) Fluorescence intensity loss for rVSV-M-Lumio-Green and $\triangle \mathrm{G}-\mathrm{rVSV}-\mathrm{M}$ Lumio-Green virions at $\mathrm{pH} 5.5$ plus or minus detergent when compared to fluorescence at $\mathrm{pH}$ 7. Samples were measured in triplicate and standard deviations are shown. 
It was previously reported that VSV released from cells expressing G on their surface could incorporate a truncated version of $\mathrm{G}$ made up of 71 amino acids that contained the transmembrane domain and cytoplasmic tail (29). It was also shown that $\Delta$ G-rVSV could incorporate a version of this protein that has the full cytoplasmic tail of $\mathrm{G}$, the transmembrane domain, and the 42 membrane-proximal amino acids on the exterior of the envelope which was named G-stem (GS) (176). Considering this protein can be found in virions that express the full-length $G$ protein (29), we wanted to assess whether or not GS could be involved in fluorescence intensity loss observed at low $\mathrm{pH}$ for rVSV-M-Lumio-Green virions. Virus containing GS and M-Lumio-Green was produced by complementing $\Delta$ G-rVSV-M-Lumio-Green virions with GS $^{\mathrm{HA}}$ (N-terminal haemaglutinin (HA) tag on GS; (94)). We also generated virus complemented with wildtype $\mathrm{G}$ (wt-G) to ensure similar virus preparations were compared. The amount of GS $\mathrm{S}^{\mathrm{HA}}$ incorporated into the complemented virus was similar to that found in virus pseudo-typed with wt-G as determined by immunoblot analysis using a monoclonal antibody to the cytoplasmic tail of $\mathrm{G}$ on an immunoblot (Fig. 3-3A). Equivalent amounts of the two viruses based on fluorescent signal were treated at $\mathrm{pH} 5.5$ and the reduction in fluorescence intensity from $\mathrm{pH} 7$ was compared. We observed a larger loss of fluorescence signal with the GS ${ }^{\mathrm{HA}}$ complemented virions versus $\Delta$ G-rVSV-M-LumioGreen virions; however the reduction was not as much as the virus with the full-length $G$ protein (Fig. 3-3B).

Low pH enhances release of $M$ protein from VSV virions. To determine if the loss in fluorescence observed when rVSV-M-Lumio-Green is exposed to low $\mathrm{pH}$ represents a biologically relevant acidification of the virion interior we asked whether internal viral proteins were affected when virus was suspended in low $\mathrm{pH}$ buffer. We hypothesized that low $\mathrm{pH}$ may be important for virus uncoating as had been shown for influenza virus (222), therefore we gently "poked holes" in the viral envelope using lysolecithin $(6,124)$, in the presence of low $\mathrm{pH}$ to allow any $\mathrm{pH}$ affected proteins on the inside of the virion to be released into the supernatant. To establish the conditions necessary to permeabilize virions without solubilizing the viral envelope, we labeled rVSV-wt virions with ${ }^{35} \mathrm{~S}$-methionine and treated them at $\mathrm{pH} 5.5$ in $\mathrm{HMN}$ buffer with different concentrations of lysolecithin and pelleted the mixture over a sucrose cushion where the released proteins would be in the supernatant and retained proteins in the viral pellet (Fig 3-4A). With the loss of G protein in lower lysolecithin concentration treatment we established appropriate conditions for our analysis (Fig. 3-4A). We also confirmed that the lysolecithin $(3.9 \mu \mathrm{g} / \mathrm{ml})$ treated virions at $\mathrm{pH} 7$ or $\mathrm{pH} 5.5$ were intact by negative stain transmission electron microscopy (TEM) (Fig 3-4B), indicating that we were observing the release of $\mathrm{M}$ from the virions rather than from disrupted RNPs. When virions were treated with either 3.9 or $7.8 \mu \mathrm{g} / \mathrm{ml}$ lysolecithin at $\mathrm{pH} 5.5$ some of the $\mathrm{M}$ protein was released and remained in the supernatant.

To determine if there was a difference in the amount of $\mathrm{M}$ released into the supernatant at $\mathrm{pH} 7$ versus $\mathrm{pH} 5.5$, we treated $1 \mu \mathrm{g}$ of virions with or without lysolecithin in HMN buffer at $\mathrm{pH} 7$ or $\mathrm{pH} 5.5$ for 10 minutes on ice and then ultracentrifuged the mixtures over a sucrose cushion to separate any released protein in the supernatant from 
A

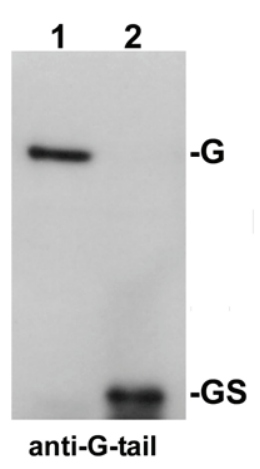

B

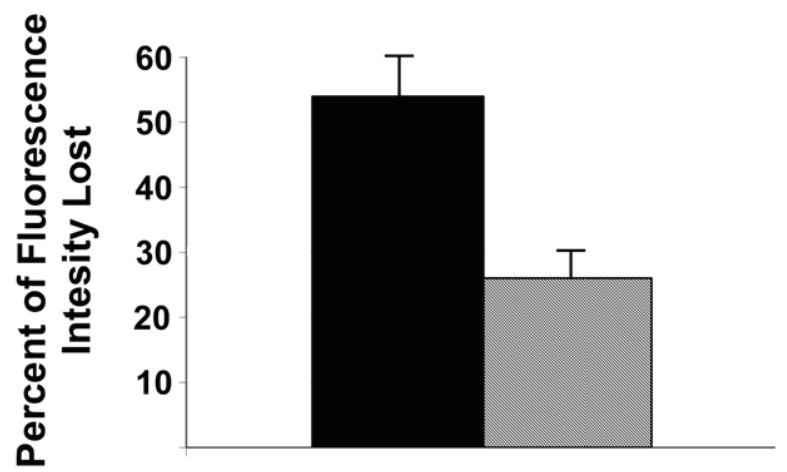

- rVSV-M-Lumio-Green

$\square \quad \mathrm{GS}^{\mathrm{HA} *} \Delta \mathrm{G}$ rVSV-M-Lumio-Green

Figure 3-3. Effect of GS ${ }^{\mathrm{HA}}$ on fluorescence intensity loss.

(A) Immunoblot using monoclonal antibody to the cytoplasmic tail of the $G$ protein to assess relative levels of $\mathrm{G}$ and $\mathrm{GS}^{\mathrm{HA}}$ incorporation into $\triangle \mathrm{G}-\mathrm{rVSV}-\mathrm{M}$-Lumio-Green virions. The amount of virus loaded for each sample was based on the fluorescence signal and 35 units of fluorescence were examined. (B) Fluorescence intensity loss for $\mathrm{G}$ and $\mathrm{GS}^{\mathrm{HA}}$ complemented $\Delta \mathrm{G}-\mathrm{rVSV}-\mathrm{M}$-Lumio-Green virions at $\mathrm{pH} 5.5$ when compared to $\mathrm{pH} 7$ fluorometer readings in triplicate and standard deviations are shown 


\section{A}

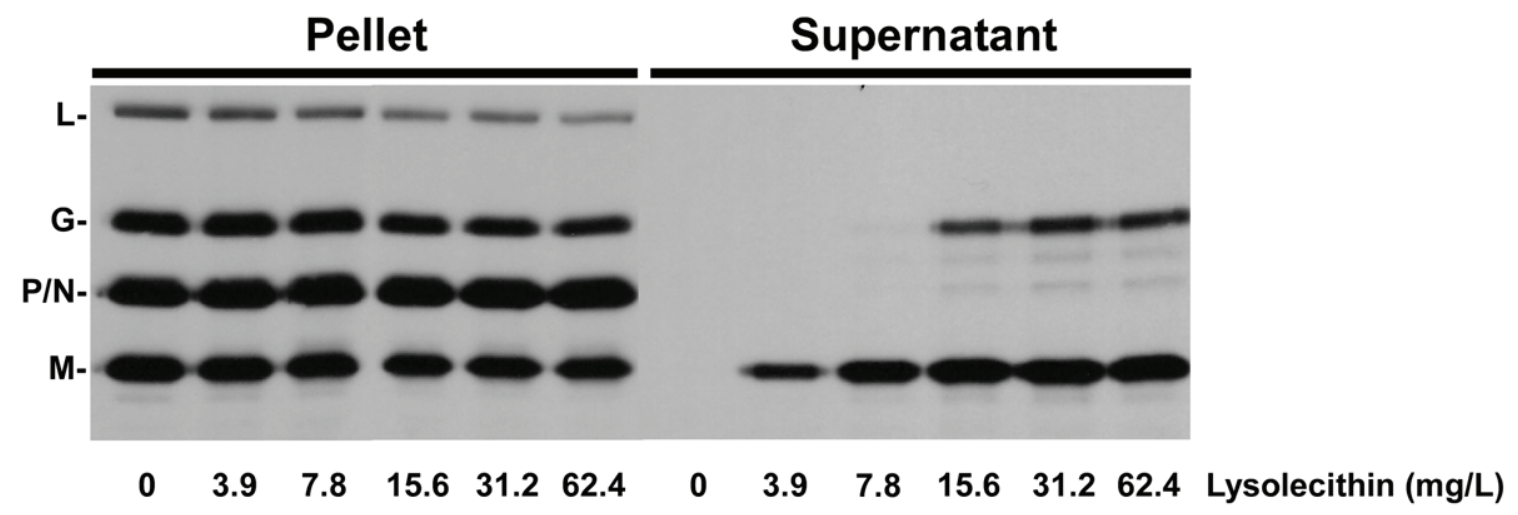

B

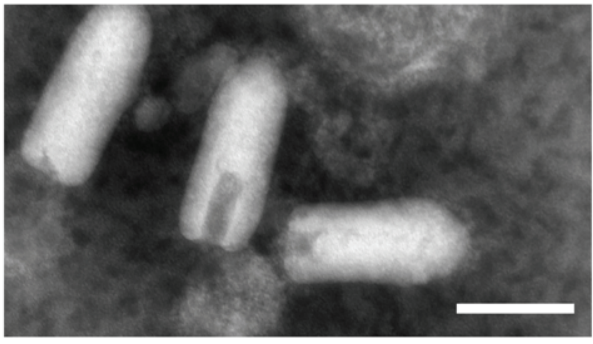

7

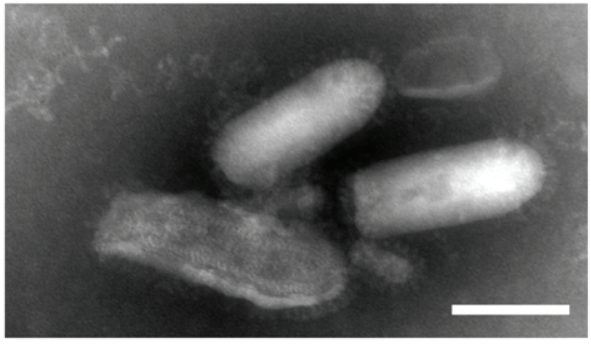

5.5

Figure 3-4. Lysolecithin treatment of rVSV-wt.

(A) Fluorograph of ${ }^{35} \mathrm{~S}$-methionine labeled rVSV-wt treated with different lysolecithin concentrations in HMN buffer at $\mathrm{pH}$ 5.5. The mixtures were ultracentrifuged over a sucrose cushion and the pellet and TCA precipitated supernatant were run on an SDSPAGE gel. (B) Transmission electron microscopy of rVSV-wt in HMN buffer at $\mathrm{pH} 7$ or $\mathrm{pH} 5.5$ treated with lysolecithin at a concentration of $3.9 \mu \mathrm{g} / \mathrm{ml}$. 100,000x Bar equals $150 \mathrm{~nm}$. 
protein retained in the virions. There was no protein release when virus was in the absence of lysolecithin at either $\mathrm{pH}$; however, we observed a difference in the amount of $\mathrm{M}$ released from lysolecithin permeabilized virus in $\mathrm{HMN}$ buffer at $\mathrm{pH} 7$ and $\mathrm{pH} 5.5$ (Fig. 3-5A). Quantification of the amount of $\mathrm{M}$ released as determined by the ratio of $\mathrm{M} / \mathrm{N}$ for virions treated with lysolecithin was $15 \%(+/-1 \mathrm{SD})$ at $\mathrm{pH} 7$ and $28 \%(+/-2 \mathrm{SD})$ at $\mathrm{pH} 5.5$ (Fig. 3-5B). Therefore, low $\mathrm{pH}$ enhanced the release of $\mathrm{M}$ protein from virions, but was not absolutely required.

\section{Discussion}

Directly after or concomitant with viral membrane fusion to the endosomal membrane, VSV M protein dissociates from the RNP core which results in the decondensation of the skeleton $(153,154)$ and the RNP is released into the host cytoplasm thereby completing uncoating (174). After uncoating, the decondensed RNP serves as a template for transcription of viral mRNAs by the packaged RNA-dependent RNA polymerase. This uncoating process is the opposite of what occurs when virus is assembled from infected cells. The exact mechanisms by which VSV can accomplish the two distinct events for the same protein are not fully understood. Uncoating of another enveloped negative-strand RNA virus, influenza virus, requires removal of its matrix protein (M1) for a productive infection $(102,137)$. The mechanism for uncoating of influenza virus has been shown to be influenced by $\mathrm{pH}$ with the M2 protein of influenza virus acting as an ion channel $(110,169)$ that allows protons across the viral envelope (81). Acidification of the virion interior decreases the interaction between M1 and nucleocapsids allowing for their dissociation before entry of the nucleocapsids into the cytoplasm (222). To understand the uncoating mechanism of VSV, we developed and characterized the virus rVSV-M-Lumio-Green which gave us the ability to follow fluorescent $\mathrm{M}$ release and distribution during entry of virions in live-cell studies (Chapter 2). rVSV-M-Lumio-Green virions were constructed with an $\mathrm{M}$ protein that has a $\mathrm{C}$ terminal fusion to a tetracysteine (-CCRECC-) Lumio $^{\mathrm{TM}}$ tag. This allowed the fluorescent labeling of $\mathrm{M}$ with the biarsenic fluorescein reagent Lumio ${ }^{\mathrm{TM}}$ Green $(2,64$, $70)$.

During live-cell synchronized entry assays we observed a loss in fluorescence intensity of rVSV-M-Lumio-Green virions during endocytosis of virus after the addition of warm media. The reduction in fluorescence signal peaked at 15 minutes post warm media addition (Fig 3-1) and then steadily increased thereafter. When we considered the $\mathrm{p} K a$ of the interaction between the fluorophore and the tetracysteine motif (70), the data suggested that the acidic environment of endosomes at $t-15$, where most of the endosomes would be near the appropriate $\mathrm{pH}$ for fusion of the viral envelope and endosomal membrane $(51,58,172)$, had an effect on the fluorescence of rVSV-MLumio-Green (Fig. 3-1B). Indeed, we observed an effect on fluorescence intensity when the $\mathrm{pH}$ was reduced from 7.0 to 5.5. In fact, the percentage of fluorescence loss seen within cells at t-15 (Fig. 3-1A, right-hand graph) was similar to the loss at $\mathrm{pH} 6$ in vitro (Fig. 3-2A). By 15 minutes the virus has likely reached an endocytic compartment with a 
Figure 3-5. Lysolecithin treatment of rVSV-wt at pH 7 and pH 5.5.

(A) Representative fluorographs of ${ }^{35} \mathrm{~S}$-methionine labeled rVSV-wt treated at a concentration of 0 or $3.9 \mu \mathrm{g} / \mathrm{ml}$ lysolecithin concentrations in HMN buffer at $\mathrm{pH} 7 \mathrm{pr} \mathrm{pH}$ 5.5. (B) Quantification of $M$ protein release as determined by the percentage of $M$ in the supernatant when compared to the amount of $\mathrm{N}$ found in the pellet. Data are the mean and standard deviation for the experiment performed in triplicate. 
A

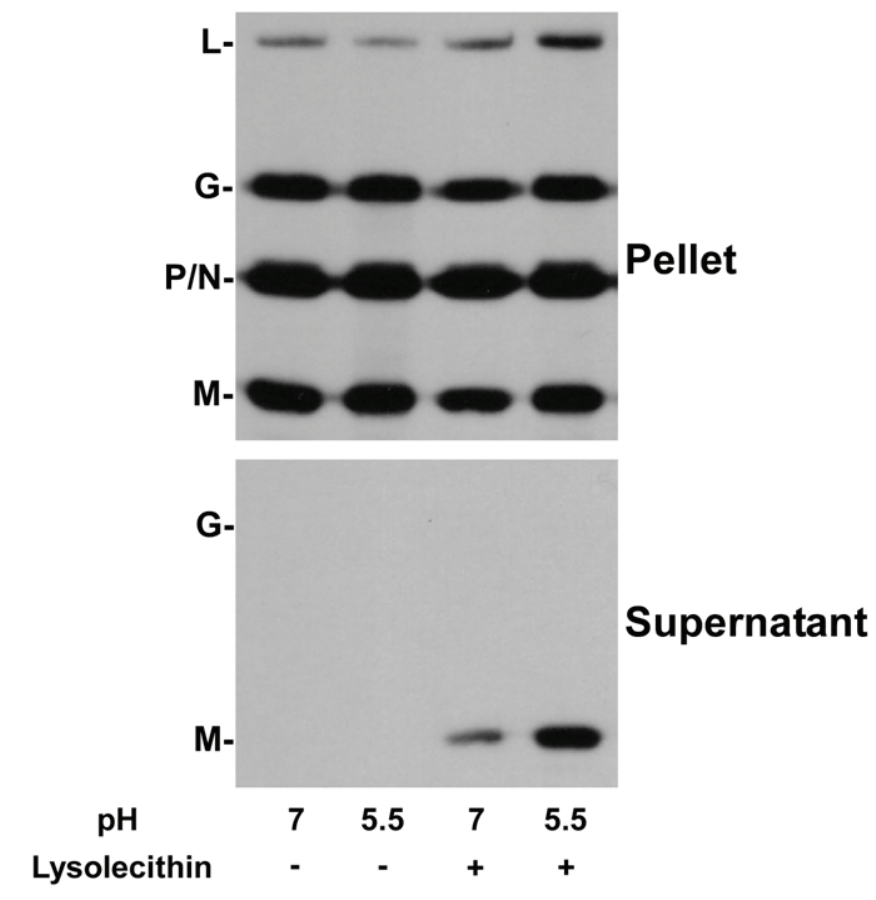

B

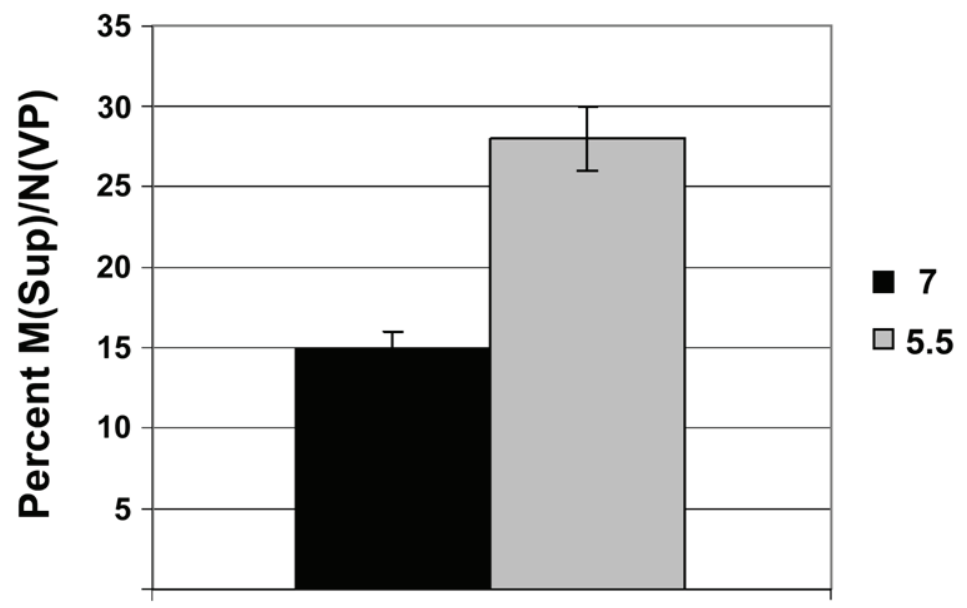


$\mathrm{pH}$ close to 6.0 (75). We also observed that the curve for fluorescence intensity loss was similar to $\mathrm{pH}$ curves for VSV G protein $\mathrm{pH}$-dependent fusion $(51,172)$ further suggesting a correlation between $\mathrm{pH}$-dependent conformation changes in $\mathrm{G}$ and the effect resulting in changes in M-Lumio-Green fluorescence. We then compared the fluorescence intensity loss of $\Delta$ G-rVSV-M-Lumio-Green and virions complemented with G (Fig. 32C) and $\mathrm{GS}^{\mathrm{HA}}$ (Fig. 3-3) in the presence of low $\mathrm{pH}$ and found that the full-length $\mathrm{G}$ protein was needed for the maximal effect of $\mathrm{pH}$ on fluorescence in vitro.

When we considered the $\mathrm{pKa}$ of $\mathrm{Lumio}^{\mathrm{TM}}$ Green when bound to the tetracysteine motif and compared the loss of fluorescence between $\mathrm{G}$, and $\Delta \mathrm{G}$ virions either treated or not treated with detergent (Fig. 3-2B), we concluded that $\mathrm{G}$ in the presence of acidic $\mathrm{pH}$ was responsible for acidification of the interior of rVSV-M-Lumio-Green virions. This was an interesting result since influenza virus uses the M2 ion channel protein for acidification of the virion interior $(110,169)$ and our results suggested that VSV uses a different method to accomplish a similar feat with its G protein. Previous studies have found spike proteins of several different enveloped viruses including VSV, Sindbis Virus (SIN), Semliki Forest virus (SFV), influenza HA, and Ebola virus GP2 when exposed to acidic $\mathrm{pH}$, can increase membrane permeability when cells expressing these proteins on the cell surface are exposed to low $\mathrm{pH}(76,101,112,182)$. The SFV spike protein's involvement in membrane disruption at low $\mathrm{pH}$ was extensively studied with cell permeabilization assays, whole-cell patch-clamp recordings, and dual voltage-clamp intracellular current flow monitoring $(112,113,115)$ and was predicted to be due to pore formation by the $\mathrm{E}_{1}-\mathrm{E}_{2}-\mathrm{E}_{3}$ protein trimers that make up the SFV spike (114). However, the mechanism of how G protein may permeabilize the VSV envelope has yet to be investigated. The $\mathrm{G}$ protein may form a pore while under going $\mathrm{pH}$-dependent conformational changes $(161,177,178)$ or rearrangement of $G$ protein trimers to the virion poles (22) may cause enough disruption of the envelope to allow protons to the interior of the virion.

The influenza virus M1 protein is affected by low $\mathrm{pH}$ within the virion which has been shown to decrease the interaction between $\mathrm{M} 1$ and nucleocapsids resulting in their dissociation before entry of the nucleocapsids into the cytoplasm (222). It has also been proposed that the capsid protein of SFV is affected by $\mathrm{pH}$ in a similar manner that would allow for efficient uncoating and association of its genome with ribosomes (114). After our observation that $\mathrm{pH}$ affected the fluorescence intensity of M-Lumio-Green within virions in a $\mathrm{G}$ protein and $\mathrm{pH}$-dependent manner (Fig. 3-2), we reasoned that exposure to low $\mathrm{pH}$ may affect the $\mathrm{M}$ proteins within VSV virions in a similar manner. It was previously shown that $\mathrm{M}$ protein exists in a dynamic equilibrium in its association with nucleocapsids and that low $\mathrm{pH}$ did not have an effect on this equilibrium (130).

Consistent with the results of that study, we found that skeletons treated with $\mathrm{pH}$ 5.5-6.0 did not release M protein from RNPs (data not shown). Even though these results suggested that $\mathrm{pH}$ has no effect on the association of the population of tightly bound $\mathrm{M}$ associated with RNPs, we reasoned that low $\mathrm{pH}$ may affect a different population of $\mathrm{M}$ inside the virion, in particular the $\mathrm{M}$ associated with the viral envelope that acts as a bridge to the nucleocapsid $(31,120,221)$ versus the $\mathrm{M}$ associated with the nucleocapsid after isolation from solubilized virions $(10,100,153)$. Indeed, virions treated with 
lysolecithin in the presence of low $\mathrm{pH}$ showed an enhanced release of $\mathrm{M}$ protein when compared to virions treated at neutral $\mathrm{pH}$ (Fig. 3-5). It is important to note that low $\mathrm{pH}$ may not be absolutely necessary for uncoating to occur since VSV pseudotypes having envelope that are $\mathrm{pH}$-independent and that fuse at the cell surface are still infectious $(95$, $142,159)$.

From this study we propose that when virions are exposed to the low $\mathrm{pH}$ environment in the endosome during entry, the contact between the $\mathrm{M}$ that bridges the viral envelope and the skeletons is reduced (30) (Fig. 3-6, green circles and black rimmed circles respectively). Acidification may trigger a slight structural change in $\mathrm{M}$ that reduces the interaction between M proteins and between the M and RNPs within the virion just before or concomitant with fusion. This reduced contact between the $\mathrm{M}$ populations would enhance the release of the skeleton into the cytosol. The $\mathrm{M}$ on the released skeleton could then dissociate away from the RNP into the M protein-less cytoplasm of newly infected cells as previously suggested (130). In summary we report a low $\mathrm{pH}$ effect on M-Lumio-Green fluorescence within virions and a low $\mathrm{pH}$ enhancement of $\mathrm{M}$ released from lysolecithin treated virions that together suggests $\mathrm{pH}$ is one of the regulatory elements involved in VSV uncoating. Although this effect is accomplished by a different mechanism in regards to influenza virus, it may indicate the involvement of low $\mathrm{pH}$ as a trigger for uncoating for many enveloped viruses that enter the host cell through the endocytic pathway. 
Figure 3-6. pH effect model.

(A) Illustration of $\mathrm{pH}$ effect on rVSV-M-Lumio-Green fluorescence and depiction of what may occur to rVSV-wt when treated with lysolecithin. (B). Model for VSV

uncoating enhanced by endosomal $\mathrm{pH}$. Acidification of the interior of virions in early endosomes affects the contact between the RNP and membrane bound M proteins (depicted by red stars) which results in the release of skeletons from membrane bound $\mathrm{M}$ protein. The release of skeletons could occur in either way or both ways depicted and then uncoating can be completed in the cytosol. 

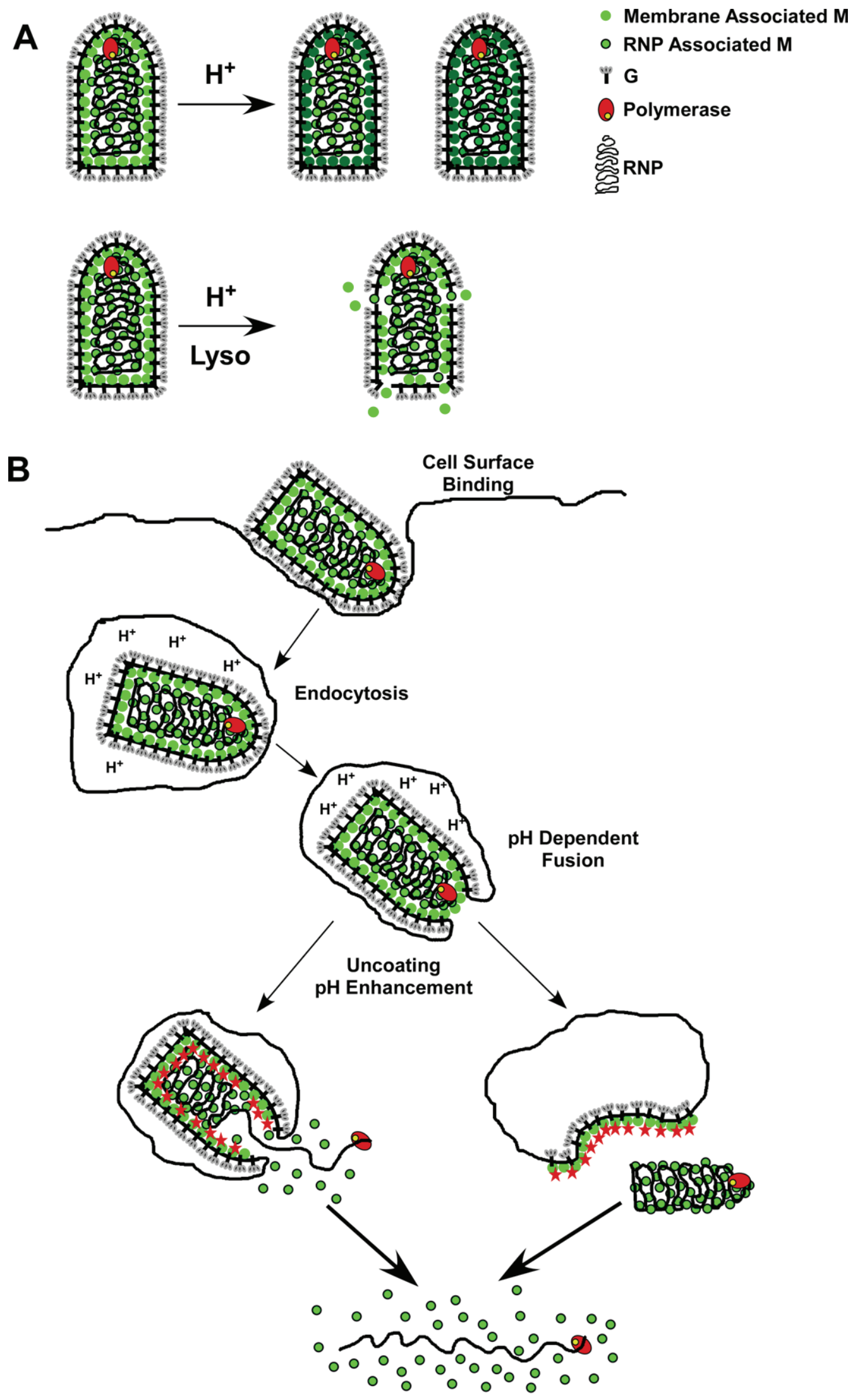

Cytosolic M Release 


\section{Chapter 4: Examination of Mutations in the Exposed Loop of the VSV Matrix Protein}

\section{Introduction}

Vesicular stomatitis virus (VSV) is a simple, enveloped, non-segmented negativestrand RNA virus that has served as a model to study virus assembly. The matrix protein (M) of VSV plays a key role in virus assembly by condensing the RNA genome and influencing the budding of virions from host cells $(30,78,91,153)$. The M protein is also involved in the cytopathic effects inflicted on host cells which include disruption of cytoskeletal organization, inhibition of host mRNA expression, translation, and nucleocytoplasmic transport $(3,4,33,53,168)$.

Biochemical studies have suggested that an exposed protease-sensitive loop (120PAVLADQGQP-129) in M may be important for assembly of virions. Cleavage of the loop with the protease thermolysin resulted in a matrix protein that no longer selfassociated $(65,66)$. M self-association has been implicated in condensation of the genome $(67,154)$ which is thought to result in inhibition of viral mRNA synthesis $(163)$ and to be critical in assembly of bullet shaped virions $(65-67,153)$. Recently M loop mutations at amino acids 121 to 124 were examined to assess their effect on assembly of virions. The assembly effect observed was minor whereas these mutations resulted in a reduction of viral protein synthesis (35). To address the involvement of the loop in virion assembly, before the report on residues 121 to 124 , we made different mutations within the loop with the hypothesis that the amino acids present (aspartate residue 125 in particular) were important for assembly. The mutants were studied in the context of $\mathrm{M}$ protein activity, and by recovery and characterization of recombinant VSV (rVSV).

Cellular distribution and budding activity of the loop mutants were comparable to wild-type. However, characterization of the mutant rVSVs revealed a reduction in growth kinetics and viral budding activity, unlike previous rVSVs with different loop mutations in $\mathrm{M}$ (35). Our results were consistent with the notion of that the loop may be involved in assembly. In addition, ${ }^{35} \mathrm{~S}$-methionine metabolic labeling studies revealed a reduction in viral protein synthesis for the rVSVs containing loop mutations from 4 to 8 hours post infection, as reported by others previously (35). Real-Time RT-PCR of cells infected with loop mutants revealed there was more viral mRNA in mutant infected cells compared to wild-type infection. A broad analysis of host responses to viral products that affect protein translation revealed that PKR activation, RNase L rRNA degradation, IFN response, apoptosis or autophagy were not involved in the reduced protein synthesis. We were able to show that this effect was most likely on a cap-dependent translation factor as translation from a Type 2 internal ribosome entry site (IRES) was not affected. These results suggest that the exposed protease-sensitive loop in M may be involved in assembly and in viral translation or that the assembly defect causes a novel host response to RNA virus infection. 


\section{Materials and Methods}

Plasmid design and construction. Construction of the cDNAs containing the loop mutations in $\mathrm{M}$ was accomplished using pBluescript(BS) M-Lumio (Chapter 2) as a vector and overlapping PCR to insert the mutations. Overlapping primers (CEM 9 and $10,11,12$ and 13,14 and 15,16 and 17,18 and 19, 20 and 21,22 and 23, used in that order for the mutations $\Delta$ Loop, 2X Loop, A Loop, D Loop, D125A, D125E, L123A, and L123S respectively) which encoded the mutations were used for PCR amplification with a 5' forward primer (CEM 1) and a 3' reverse primer (T3) respectively. Another round of PCR amplification with the overlapping 5' and 3' fragments as the template and the two outside (CEM 1 and T-3-reverse) were used to create the desired mutations in the MLumio gene. The PCR product was gel purified, digested with AscI and EagI, gel purified, and ligated into pBS-SK- $\Phi$ T cut with the same enzymes.

Generation of full-length VSV cDNAs encoding M loop mutants. Creation of the full-length VSV genomes with M loop mutants was accomplished by separately cloning each sequence into a $\mathrm{p} \triangle \mathrm{M}-\mathrm{PLF}$ VSV anti-genome which has a multiple cloning site in the place of the M protein coding sequence (91), except GFP is not encoded in the plasmid. For each construct the plasmids were digested with $A s c \mathrm{I}$ and $E a g \mathrm{I}$, the $\Delta \mathrm{M}$ vector and $\mathrm{M}$ loop mutant fragments were gel purified and ligated by two way ligation to create $\mathrm{pVSV}$-(Loop Mutation).

Recovery and characterization of recombinant VSV (rVSV). Recombinant viruses were recovered using reverse genetics (116) with some modifications as described previously (91). Single-step growth curves were performed by adsorbing virus to baby hamster kidney (BHK-21) cells in $35 \mathrm{~mm}$ plates at a multiplicity of infection (MOI) of 10 for 1 hour at $31^{\circ} \mathrm{C}$ while continually rocking. The inoculum was removed, the cells were washed $4 \mathrm{x}$ with serum-free Dulbecco's minimal Eagle's medium (DMEM) to remove unbound virus, DMEM containing $5 \%$ fetal bovine serum (FBS) was added, and the cells were placed at $37^{\circ} \mathrm{C}$. Every two hours post infection (hpi) a $5 \%$ aliquot of media was collected, replaced with the same volume of fresh media, and then virus titers were determined in duplicate by plaque assay on BHK-21 cells. Growth curves were performed in triplicate for each virus. Relative infectivity was assessed by Coomassieblue staining after SDS-PAGE analysis of $1 \times 10^{7} \mathrm{PFU}$ of rVSV.

TEM of rVSV. To confirm the morphology of rVSV, purified virus was resuspended in $10 \%$ sucrose $10 \mathrm{mM}$ Tris- $\mathrm{HCl}$ [pH 8.0], $150 \mathrm{mM} \mathrm{NaCl}$ solution and placed on ice. To decrease the sucrose concentration, the virus preparations were then diluted 1:10 in $\mathrm{dH}_{2} \mathrm{O}$ and prepared for transmission electron microscopy (TEM) by adsorbing virus to a carbon-coated grid using the drop-to-drop method and negative staining with $2 \%$ phosphotungstic acid (PTA, pH 5.9). 
Transient expression of $\mathbf{M}$ loop mutants. BHK-21 cells at $\sim 90 \%$ confluency on glass cover slips, were infected with a modified vaccinia virus (vTF7-3) encoding T7 RNA polymerase (61) at MOI 5 for 1 hour at $31^{\circ} \mathrm{C}$ in SF-DMEM. The inoculum was removed and the cells were transfected with $5 \mu \mathrm{g}$ of pBS-MФT (wild-type; Indiana) or pBS-(Loop Mutant) using $20 \mu 1$ TransfectACE in SF-DMEM (180). Five hours post transfection (p.t.) the transfection mix was removed and replaced with 5\% FBS DMEM containing antibiotics (100U/ml streptomycin and penicillin), and 18 hours p.t. the cells were fixed and processed for immunofluorescence or were used in a budding assay as described below.

Immunofluorescence (IF) staining and confocal microscopy. Cells either transiently expressing M proteins from plasmids or infected with rVSV were washed twice with phosphate-buffered saline (PBS) and then fixed for 15 minutes with 3\% paraformaldehyde (PFA) in PBS at room temperature (r.t.). The fix solution was then removed and the cells washed twice with PBS containing $10 \mathrm{mM}$ Glycine and $0.05 \%$ sodium azide (PBS-Glycine), and then permeabilized with $1 \%$ Triton X-100 in PBSglycine at r.t. for 1 minute. After permeabilization, the cells were washed twice with PBS-Glycine and then stained for; $M$ protein using an anti-M monoclonal antibody (mAb 23H12) conjugated to FITC or rhodamine, VSV N protein using anti-N mAb 10G4 conjugated to rhodamine., or microtubules using anti- $\alpha$ tubulin mAb (\# A-11126, Molecular Probes). The unconjugated primary antibodies were detected with a goat antimouse secondary antibody conjugated to rhodamine (Jackson Research Laboratories). Phalloidin conjugated to Texas Red-X (Invitrogen; Molecular Probes) was used to stain actin. The distribution of the indicated proteins was examined using laser scanning confocal microscopy (Zeiss LSM 510). Optical slices of $1 \mu \mathrm{m}$ were captured using $488 \mathrm{~nm}$ or $543 \mathrm{~nm}$ laser excitation.

Budding assays. Cells transiently expressing the indicated $\mathrm{M}$ protein were washed twice with methionine-free, SF-DMEM and then incubated in this media for 15 minutes to deplete the intracellular methionine pools at 5 hours p.t. After depletion, a media composed of 1 part 5\% FBS DMEM to 9 parts methionine-free media and supplemented with $50 \mu \mathrm{Ci}$ of ${ }^{35} \mathrm{~S}-$ Methionine (Met) Express Protein Labeling Mix (Perkin-Elmer) per ml was added and 18 hours later media and cells were collected separately and processed for immunoprecipitation (IP).

The media were centrifuged at $170 \mathrm{x}$ g for 10 minutes to remove dislodged cells and the supernatant was placed on ice after addition of $200 \mathrm{U}$ of aprotonin (U.S. Biochemicals). The supernatant was made to 50mM Tris-HCl, $150 \mathrm{mM} \mathrm{NaCl}, 0.1 \% \mathrm{NP}-$ 40, $1 \mathrm{mM}$ EDTA, $20 \mathrm{mM} \mathrm{NaN}_{3}, 0.3 \% \mathrm{SDS}$, and radio labeled $\mathrm{M}$ proteins were immunoprecipitated with $\mathrm{M}$ mAb (23H12) overnight at $4^{0} \mathrm{C}$.

Cell extracts were prepared by washing the cells twice with PBS and then lysing with $800 \mu \mathrm{l}$ of detergent solution (10 mM Tris- $\mathrm{HCl}$ [pH 7.4], $66 \mathrm{mM}$ EDTA, 0.4\% sodium deoxycholate, $1 \%$ Triton $\mathrm{X}-100,0.05 \% \mathrm{NaN}_{3}$ ) containing $200 \mathrm{U} / \mathrm{ml}$ of aprotonin 
for 5 minutes at r.t. on a rocker. The cell extract was collected, nuclei and insoluble material removed by centrifugation at $10,000 \mathrm{x}$ g for 5 minutes and a $200 \mu \mathrm{l}$ aliquot of the cell extract was made to $0.3 \%$ SDS and immunoprecipitated with $\mathrm{M} \mathrm{mAb}(23 \mathrm{H} 12)$ overnight at $4^{\circ} \mathrm{C}$. One-quarter of the radio labeled $\mathrm{M}$ proteins in the cell extract were compared to the amount released into the supernatant by SDS polyacrylamide gel electrophoresis (PAGE) on a 9\% polyacrylamide gel and visualized by fluorography.

Immunoblot analysis. To detect the proteins in BHK-21 or HeLa cells that were mock treated/infected, rapamycin treated, or infected with $\mathrm{rVSV}$ cell lysates from $\sim 10^{6}$ cells were collected at different times post infection by addition $400 \mu \mathrm{l}$ of detergent solution (10 mM Tris- $\mathrm{HCl}$ [pH 7.4], $66 \mathrm{mM}$ EDTA, $0.4 \%$ sodium deoxycholate, $1 \%$ Triton $\mathrm{X}-100,0.05 \% \mathrm{NaN}_{3}$ ) containing $200 \mathrm{U} / \mathrm{ml}$ of aprotonin. The cell extracts $(5 \%)$ were separated by SDS-PAGE on a $10 \%$ polyacrylamide gel and then transferred to a polyvinylidene fluoride (PVDF) microporous membrane (Immobilon-P; Millipore). Monoclonal antibodies to M (23H12), $\alpha$ tubulin (\# A-11126, Molecular Probes), eIF2 $\alpha$ (\# 9722, Cell Signaling Technologies (CST)), phosphorylated eIF2 $\alpha$ (\# 9721, CST), caspase 3 (\# 9668, CST), phosphorylated eIF4G (\# 2441, CST), and LC3B (\# 2775, CST) were used to detect each protein followed by addition of a goat anti-mouse or -rabbit antibody tagged with horseradish peroxidase (Jackson Research Laboratories). Proteins were visualized using a chemiluminescence kit (Immobilon Western; Millipore) according to the manufacturer's instructions.

${ }^{35}$ S-methionine pulse labeling of $\mathbf{r V S V}$ infected cells. BHK-21 cells were infected with rVSV at an MOI of 10. At increasing times post infection the cells were starved of methionine for 10 minutes and then an ${ }^{35}$ S-methionine labeling mix at $50 \mu \mathrm{Ci} / \mathrm{ml}$ was added to the cells for 10 minutes. Cell extracts were prepared by washing the cells twice with PBS and then lysing with $400 \mu$ lof detergent solution $(10 \mathrm{mM}$ Tris$\mathrm{HCl}$ [pH 7.4], $66 \mathrm{mM}$ EDTA, $0.4 \%$ sodium deoxycholate, $1 \%$ Triton X-100, $0.05 \%$ $\mathrm{NaN}_{3}$ ) containing $200 \mathrm{U} / \mathrm{ml}$ of aprotonin for 5 minutes at r.t. on a rocker. The nuclei and insoluble material were removed by centrifugation at $10,000 \mathrm{x}$ for 5 minutes and $5 \%$ of the cell extract was analyzed by SDS-PAGE fluorography and quantified by phosphorimaging using a STORM (Molecular Dynamics) scanner.

rVSV budding assay. BHK-21 cells were infected with rVSV at an MOI of 10. At $3 \mathrm{hpi}$, cells were starved of methionine for 10 minutes and then an ${ }^{35} \mathrm{~S}$-methionine labeling mix at $50 \mu \mathrm{Ci} / \mathrm{ml}$ was added to the cells for 1 hour. The supernatant was then collected and virus was isolated from the supernatant by ultracentrifugation ( 35 minutes, $45 \mathrm{~K} \mathrm{rpm}$, Sorvall, AH-650) over a 20\% sucrose TN (10 mM Tris-HCl [pH 8.0], $150 \mathrm{mM}$ $\mathrm{NaCl}$ ) cushion. Also, cell extracts were prepared by washing the cells twice with PBS and then lysing with $400 \mu 1$ of detergent solution (10 mM Tris-HCl [pH 7.4], $66 \mathrm{mM}$ EDTA, $0.4 \%$ sodium deoxycholate, $1 \%$ Triton $\mathrm{X}-100,0.05 \% \mathrm{NaN}_{3}$ ) containing $200 \mathrm{U} / \mathrm{ml}$ of aprotonin for 5 minutes at r.t. on a rocker. The nuclei and insoluble material were removed by centrifugation at $10,000 \mathrm{x}$ g for 5 minutes. The whole viral pellet and $5 \%$ of 
the cell extract were analyzed by SDS-PAGE fluorography and quantified by phosphorimaging using a STORM scanner.

Synchronized fusion co-infection assay. To prevent the acidification of endosomes and synchronize fusion of virions with endosomal membranes we used the lysosomotropic reagent $\mathrm{NH}_{4} \mathrm{Cl}$ as described previously (174) with some modifications. BHK-21 cells were washed twice with PBS and then washed twice with PBS containing $100 \mathrm{mM} \mathrm{NH}_{4} \mathrm{Cl}$. rVSV (MOI 1), rVSV-D125A (MOI 10), or rVSV-wt (MOI 1) and rVSV-D125A (MOI 10) were adsorbed in SF-DMEM containing $100 \mathrm{mM} \mathrm{NH}_{4} \mathrm{Cl}$ for 90 minutes. After adsorption the cells were washed with PBS four times to remove the $\mathrm{NH}_{4} \mathrm{Cl}$ and protein synthesis or virus budding was examined by ${ }^{35} \mathrm{~S}$-methionine pulse labeling as described above.

RNP exchange assay. ${ }^{35}$ S-methionine labeled M-wt or M D125A mutant protein was made from plasmids in a TNT T7 in vitro transcription and translation reaction (\# L4610, Promega) in the presence of ${ }^{35}$ S-methionine labeling mix (\# AG1594, GE Healthcare). Five microliters of either translation reaction was added to 10, 50, or $100 \mu \mathrm{g}$ of solubilized rVSV-wt or rVSV-D125A in TN (Tris-HCl (pH 8.0), 150mM NaCl) buffer. The mixtures were placed on ice for 10 minutes and then the RNPs and supernatants were separated by ultracentrifugation $(35$ minutes, $45 \mathrm{~K} \mathrm{rpm}$, Sorvall, AH650 ) over a $20 \%$ sucrose TN cushion. Pellets and TCA precipitated supernatants were then analyzed by SDS-PAGE fluorography.

RT-PCR analysis. To rule out viral $\mathrm{mRNA}$ as the limiting factor in the reduction in protein synthesis during infection, real-time RT-PCR was performed on BHK-21 cells infected with rVSV at an MOI of 10 and at increasing times post infection cell lysates were collected in Trizol reagent (\# 15596-018, Invitrogen) and total RNA was extracted. Reverse transcription was performed using a primer (MR Nest) on a known amount of genomes from $10^{1}$ to $10^{13}$ to produce a standardized genomic curve for RT-PCR. Reverse transcription was also performed on total RNA extracted from cells using a poly dT primer for mRNA and a primer (MR Nest) that annealed to the intergenic region between $\mathrm{M}$ and $\mathrm{G}$ for genomes. RT-PCR was then performed and RNA was quantified using SYBR Green for genomes (primers CEM 2 and MR Nest) and P mRNA (P82F and $\mathrm{P}-210-\mathrm{REV}$ ) in a DNA Engine Opticon system and software (MJ Research/BioRad).

Luciferase assays. HeLa cells were mock transfected, transfected with a nonreporter plasmid, or $125 \mathrm{ng}$ of a plasmid that encoded Firefly luciferase under the control of the IFN $\beta$ promoter ( $p$ IFN $\beta$-Luc) that expressed luciferase. Five hours post transfection of IFN $\beta$-Luciferase plasmid cells were mock treated/infected, treated with $125 \mathrm{U}$ of human IFN, or infected with rVSV-wt or rVSV-D125A at an MOI of 10. Cell lysates were collected at increasing times post infection and analyzed using a luciferase assay system (\# E1500, Promega) in a luminometer (Turner Designs, TD-20/20). 
HeLa cells were also transfected with $2 \mu \mathrm{g}$ of a plasmid that expressed luciferase from a chicken actin promoter (pCAAGs-Luc) and at 5 hours post transfection were mock infected or infected with rVSV-wt or rVSV-D125A at an MOI of 10 and cell lysates at increasing times post infection were analyzed for luciferase activity as described above.

BSR cells (BHKs constitutively expressing T7 RNA polymerase, (179)) were transfected with $5 \mu \mathrm{g}$ of a plasmid that expressed luciferase with an internal ribosome entry site (pEMCV/IRES-Luc) and 18 hours post transfection the cells were mock infected/treated, treated with cycloheximide $(10 \mu \mathrm{g} / \mathrm{ml})$, or infected with $\mathrm{rVSV}$-wt or rVSV-D125A at an MOI of 10. Mock and cycloheximide treated cell lysates were assessed for luciferase activity at 6 hours post treatment and luciferase activity was measured at increasing times post infection in infected cells as described above.

\section{Results}

\section{Recovery and characterization of recombinant (r)VSV with M loop}

mutations. To investigate the role that the amino acids within the protease-sensitive loop of $\mathrm{M}$ protein may play in virus assembly we constructed $\mathrm{M}$ proteins with mutations in the loop (Fig. 4-1A). All of the constructs included a C-terminal Lumio tag, which we have shown does not affect $\mathrm{M}$ protein localization, budding activity, or uncoating when assembled into virus particles (Chapter 2). Figure 4-2A shows an alignment of the putative loop regions of vesiculovirus $M$ proteins showing that the aspartate residue (highlighted) is also conserved. To determine if the loop in $\mathrm{M}$ had conserved sequences we performed a PubMed blast search of the virus protein database and Figure 4-2B shows the results that revealed an LXD sequence for other virus proteins. Based on these alignments (Fig. 4-2), the mutations in Figure 4-1A were constructed to determine whether the loop ( $\Delta$ Loop), size of the loop (2X Loop), or specific amino acids were involved in the assembly and/or uncoating of VSV.

To assess the cellular distribution and assembly function of these $\mathrm{M}$ proteins with loop mutations, we transiently expressed these proteins in cells and compared them to $\mathrm{M}$ wt by immunofluorescence microscopy (IF)) and in a budding assay (Figs.4-1B and C). There were no discernable difference in the intracellular distributions of the M loop mutants as they all were located in the nucleus, were cytosolic, and localized to the plasma membrane as determined by reactivity with a monoclonal anti-M antibody conjugated to rhodamine (Fig. 4-1B). The loop mutants also had wild-type budding activity (Fig. 4-1C lane 6). Note D125A is the only mutant shown in the IF and budding assay but the other loop mutations retained similar wild-type activity.

To determine if $\mathrm{M}$ loop mutants could support virus assembly we replaced the Mwt gene with the loop mutants in the viral genome (Fig. 4-1A). Only the viruses with single amino acid mutations (D125A, L123A, and L123S) were recovered. Although we did not recover the D125E mutant from plasmids, it was obtained as a "revertant" from 
Figure 4-1. Design and characterization of the $M$ loop mutant proteins.

(A) Schematic of mutations made in the protease-sensitive loop region of $\mathrm{M}$ and their location within rVSV genomes. The residues in grey color and underlined are either mutated to the amino acids shown, or are added (2X Loop). The -PP-indicates deletion of the loop region ( $\triangle$ Loop) between the two prolines at residues 121 and 129 in the wildtype sequence $(\mathrm{Mwt})$. All constructs contained a tetracysteine Lumio ${ }^{\mathrm{TM}}$ tag. (B) Transient expression of Mwt and the D125A loop mutant in BHK-21 cells stained using an anti-M monoclonal antibody conjugated to rhodamine and examined by LSM using a $100 \mathrm{X}$ magnification objective and $543 \mathrm{~nm}$ excitation. Image is a $1 \mu \mathrm{m}$ optical section through the middle of the cell. (C) Budding assay showing intracellular protein expression (odd numbered lanes) and budding activity in mock transfected (lane 2), Mwt (lane 4), and D125A (lane 6) transfected cells. 
A

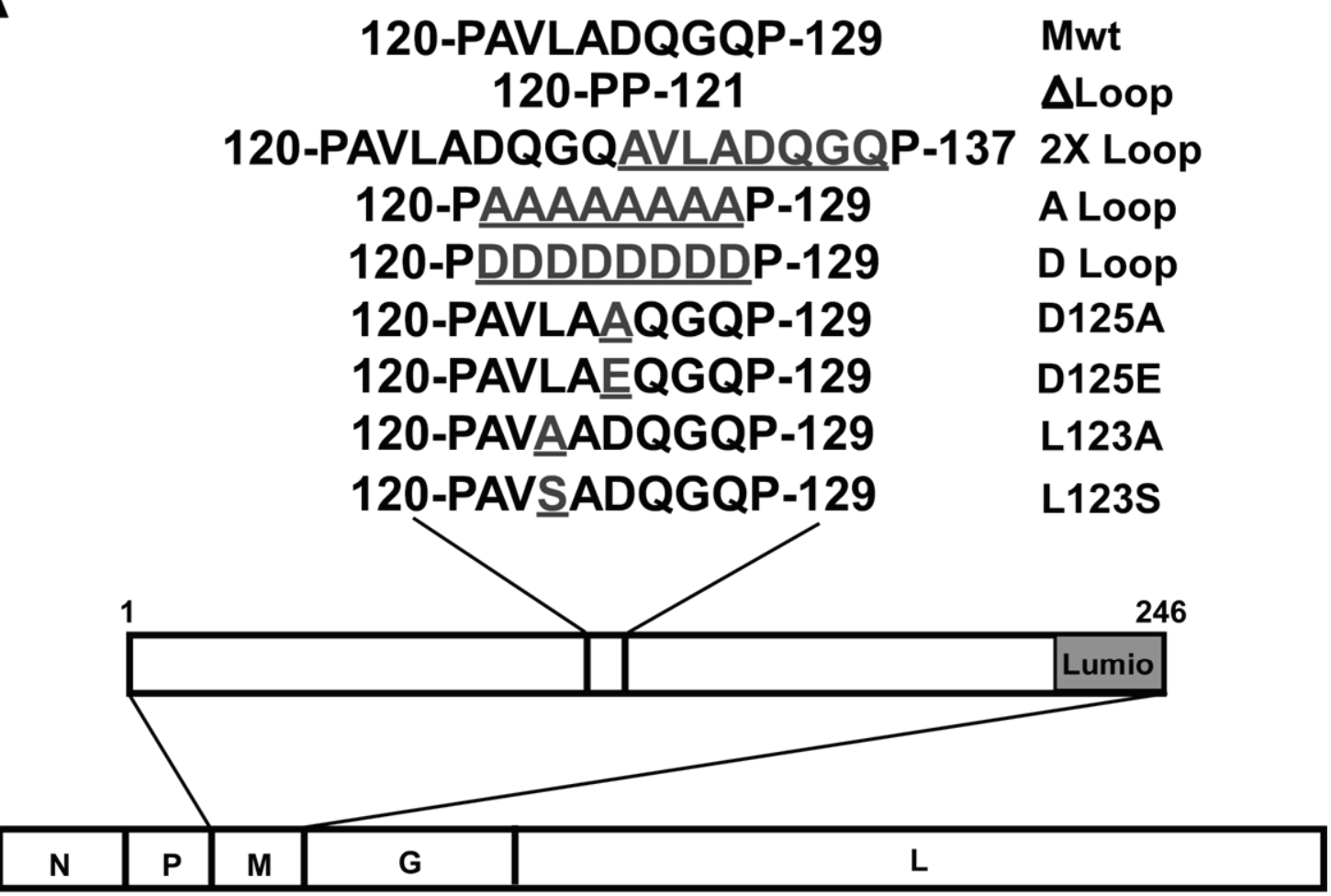

B

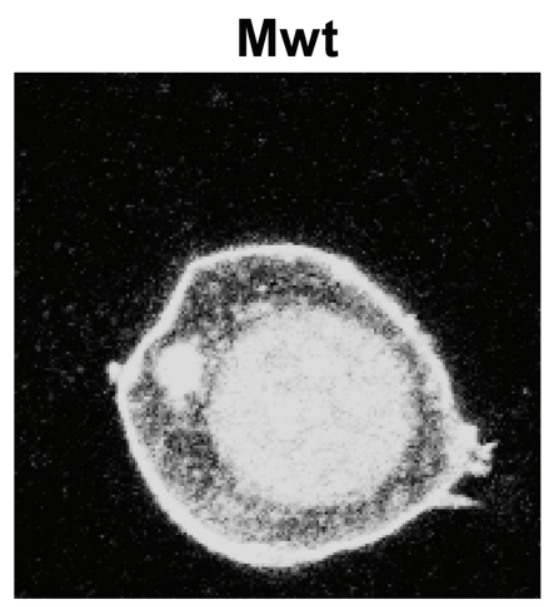

D125A

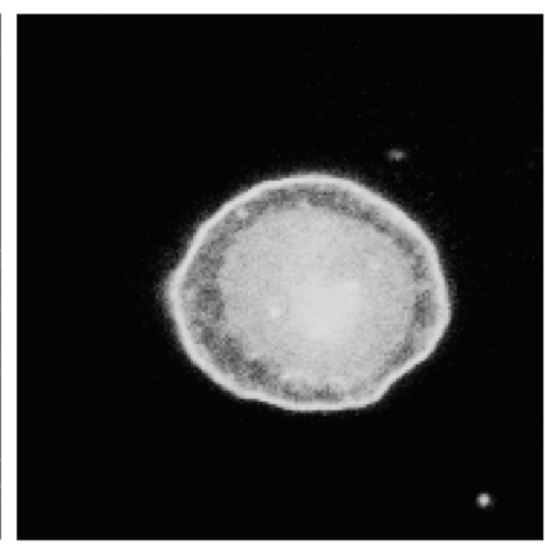

C

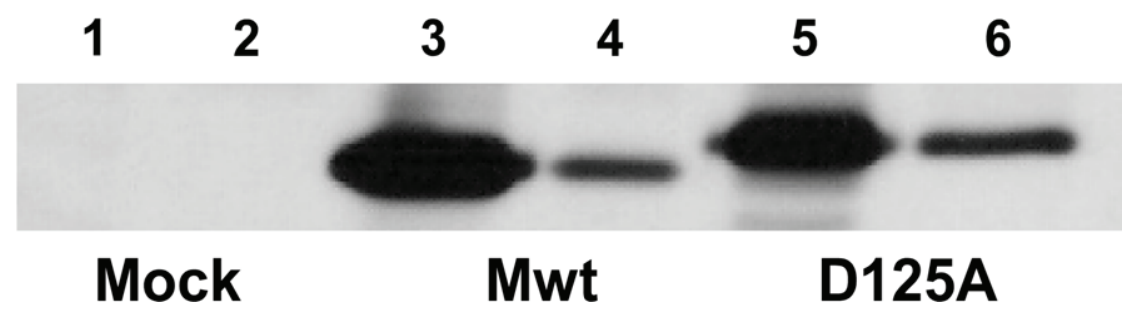




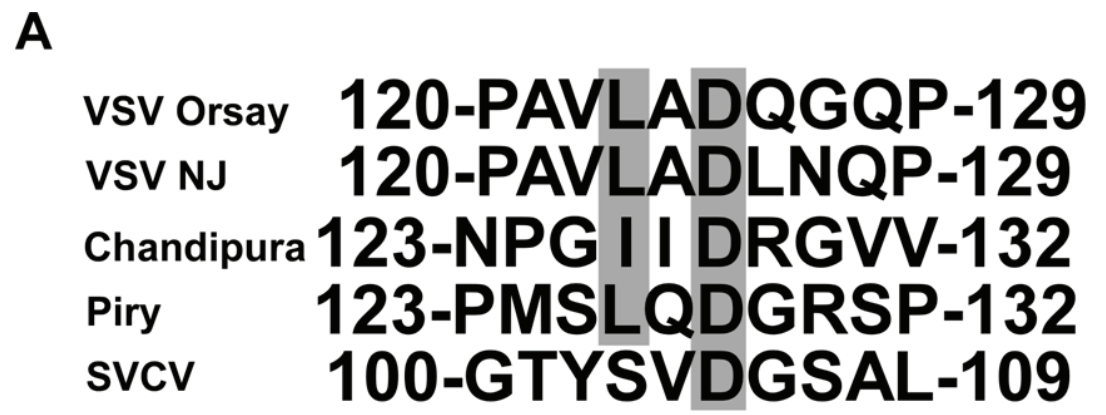

B

\begin{tabular}{lcc}
\hline \multicolumn{1}{c}{ Virus Protein } & LXD Sequence \\
\hline VSV Matrix & & $120-$ PAVLADQGQP-129 \\
Sendai virus Matrix & 220-VLDDQGE-226 \\
Rice stripe virus (T) Coat protein & $6-P A T L A D-11$ \\
SV5(w3) Nucleoprotein & & 79-PSATMLNVKLADQSP-95 \\
Turkey rhinotracheitis virus Matrix & $44-P A P V L A D Q---P-65$ \\
HSV-I(17) Capsid assembly protein (UL37) & & $612-P N V L A D P-618$ \\
Catfish herpesvirus gene 6 membrane protein & & 18-AVLAD-22 \\
Rice dwarf virus P2 outer Capsid protein & & 88-AVLAD-92 \\
Human herpsvirus 4 Capsid assembly protein & & 162-PATLAD-167 \\
Mammalian orthoreovirus 3 Core protein (Sigma 2) & $360-$-LADQTQ-365 \\
\hline
\end{tabular}

Figure 4-2. M loop sequence alignments.

(A) Zoomed in view of predicted loops from matrix proteins of different Vesiculoviruses showing the Leucine/Isoleucine and aspartate (L/I and D respectively, highlighted) residue and their location in the sequences. Alignment of matrix proteins from Gaudier et al. (66) using Clustal W was used to align the predicted loops. (B) Alignments and results of a PubMed blast search for the VSV M loop sequence, revealing an LXD sequence in proteins involved in some form of assembly from different viruses. 
rVSV-D125A after 7 passages on $10^{7}$ cells using an MOI of 0.001 at each passage. As described below, D125A forms very small plaques and does not cause cell rounding (Fig. 4-2A). The D125E mutation is referred to as a revertant since it restored (data not shown) the cell rounding activity that was lost in the D125A mutant phenotype when compared to wild-type (Fig. 4-3A). This phenotype was a significant observation since BHK-21 cells, which are highly susceptible to VSV cytopathic effects (CPE), did not round when infected with D125A. A previous study by others showed that infection of MDCK cells with a rVSV M loop mutant (121-DKQQ-124, rVSV-ФM) did not show a wild-type cell rounding but the mutant did in BHK cells (35). To determine if there were differences in the disruption of the actin- or tubulin-based cytoskeleton within cells infected with wild-type or the D125A mutant, we examined infected cells by laser scanning confocal microscopy after staining for $\mathrm{M}$ and either tubulin or actin at 8 hours post infection (hpi). We found that tubulin was not disrupted at this time post infection (Fig. 4-3B) while actin was reorganized in wild-type infected cells, but not in D125A infected cells (Fig. 4-3C). The result we observed was interesting since previous studies using different microscopy and methods had linked the disorganization of microtubules as the source for virus induced cell rounding $(150,190)$.

To determine if the growth kinetics of the M loop mutants were similar to rVSVwt we performed single-step growth curves for each virus. All the mutants grew more slowly and had a titer of one log less than wild-type at 10hpi (Fig. 4-4A). To assess the relative infectivity of the virions, proteins from $10^{7} \mathrm{PFU}$ of the rVSV-D125A and rVSVwt virions were separated by SDS-PAGE and Coomassie-blue stained (Fig. 4-4B lanes 1 and 2, respectively). The protein compositions from equivalent amounts of infectious virus were similar indicating the reduced infectivity for D125A found at $10 \mathrm{hpi}$ in the one-step growth curve analysis was due to less virus being assembled and released from infected cells. We then compared the morphology of purified virions by transmission electron microscopy using negative staining. As seen with the relative infectivity analysis, the morphologies of the D125A loop mutant and rVSV-wt (Wt) were both bullet-shaped with helically condensed RNPs (Fig. 4-4C). These results further supported the conclusion that the the reduction in growth kinetics of the loop mutants was due to an assembly defect.

rVSV M loop mutants show reduced viral protein synthesis at later times post infection. Previously it was shown that an rVSV M loop, rVSV- $\Phi M$, had a defect in viral protein synthesis (35) without significant assembly defects. To determine if our rVSV M loop mutants had similar defects in viral protein translation we infected cells at an MOI of 10 pulse-labeled the cells with ${ }^{35} \mathrm{~S}$-methionine for 10 minutes at 6 hpi and analyzed the labeled proteins by SDS-PAGE and phosphorimaging (Fig. 4-5). For each gel lane 1 shows the protein synthesis of mock infected cells. Quantitation of the difference in viral protein synthesis between wild-type and the D125(E or A) loop mutants (Fig. 4-5A lanes 2, 3, and 4, respectively) or the L123(A or S) mutants (Fig. 45B lanes 3 and 4, respectively) is represented in the graphs and shows a larger reduction in viral protein synthesis for the D125A mutant. The rVSV M loop mutants showed similar reduced growth kinetics to each other (Fig. 4-4A), but the D125A mutant had a 
Figure 4-3. Cell rounding of rVSV-wt and rVSV-D125A infected cells.

(A) BHK-21 cells were infected with wild-type (Wt) and mutant virions (D125A) at an MOI of 10 and phase-contrast images were taken at 24 hours post infection (hpi) showing reduced cell rounding in rVSV-D125A infected cells. (B and C) Cells at 4 hpi were fixed, permeabilized, and stained for $\mathrm{M}(\mathrm{Ex} .488 \mathrm{~nm})$ and tubulin (B) or actin (C) (Ex. $543 \mathrm{~nm})$, showing that reorganization of actin $(\mathrm{C}, \mathrm{Wt})$ correlated with cell rounding in rVSV-wt infected cells. 

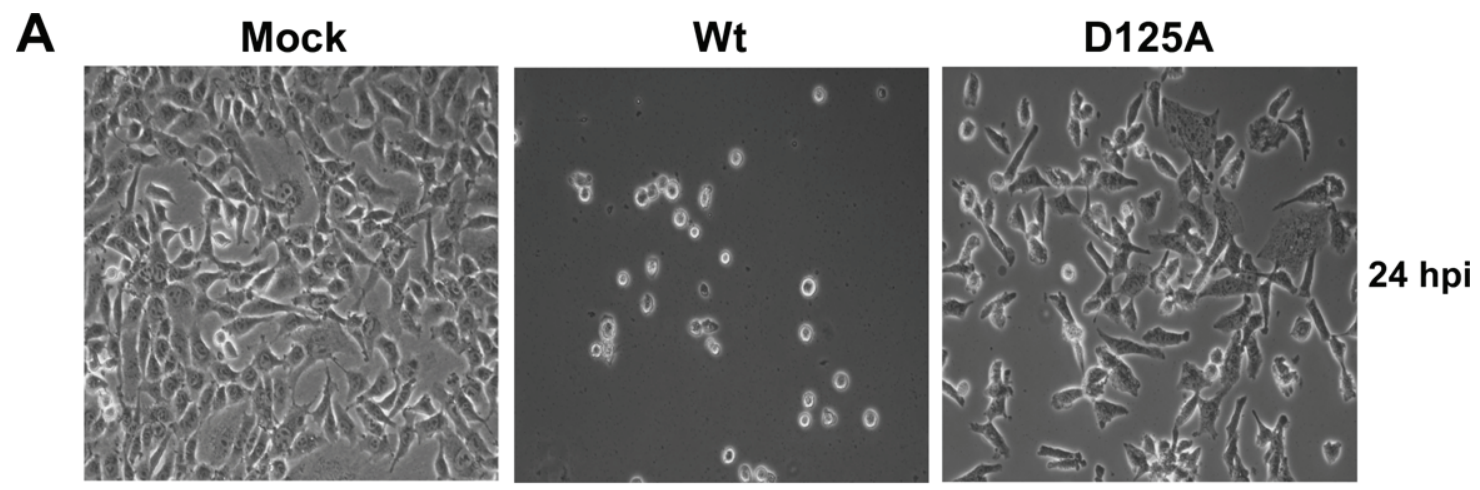

B

Ex. 488nm
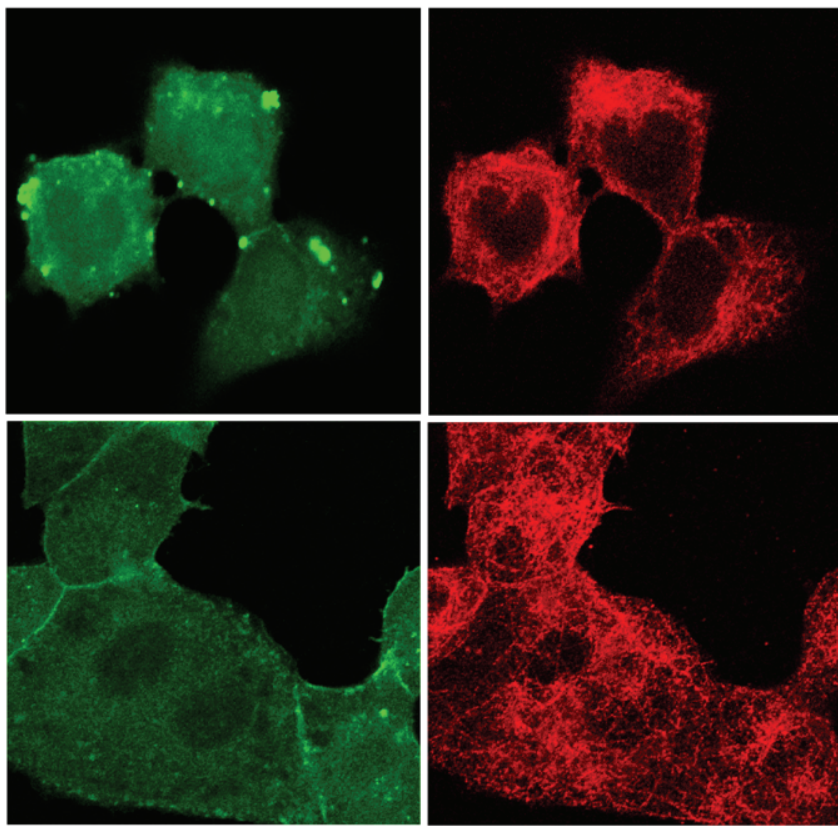

Wt

D125A

C
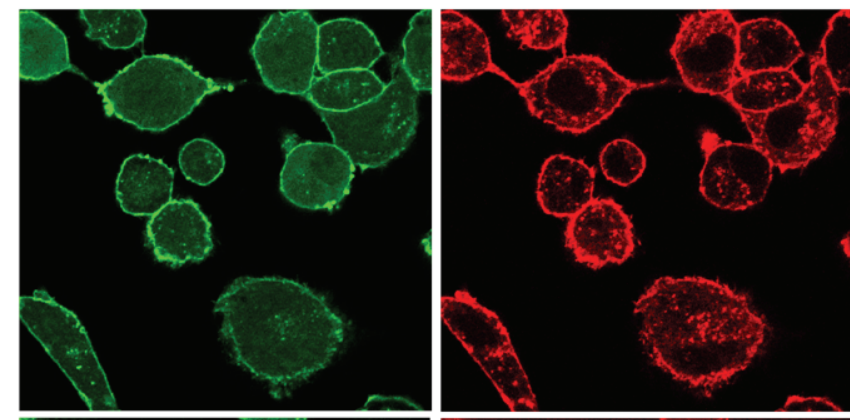

Wt
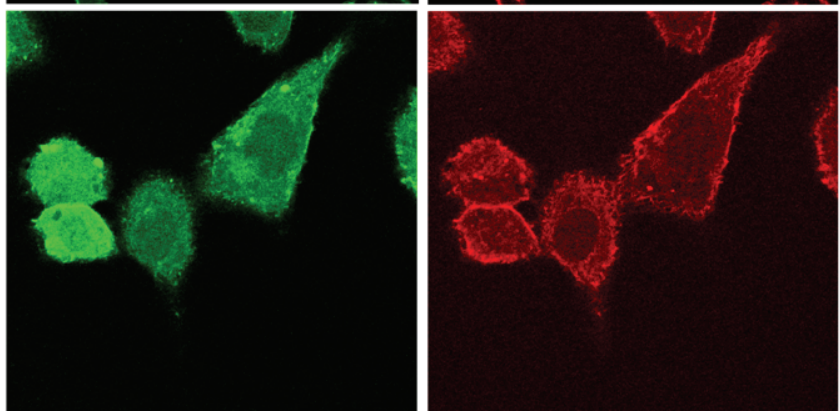

D125A 

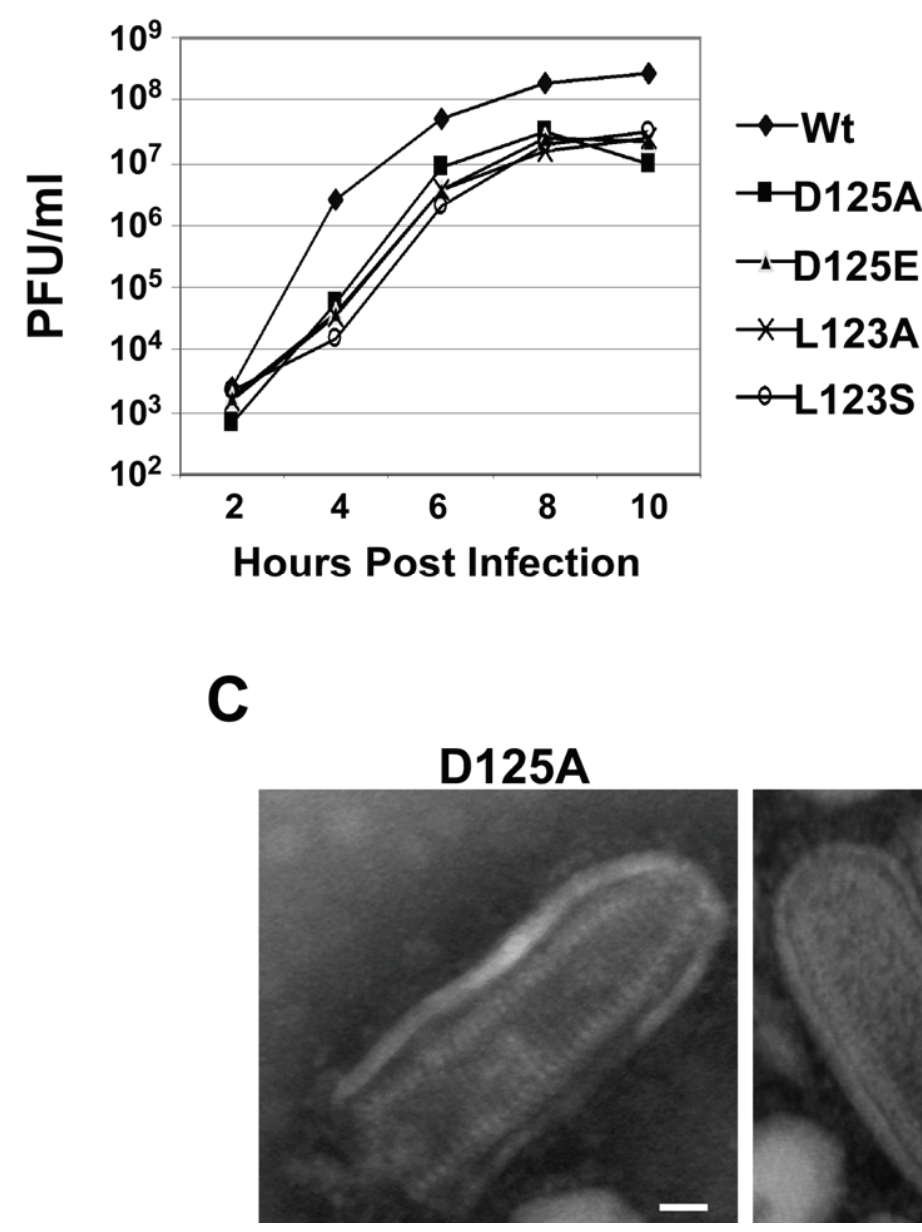

B

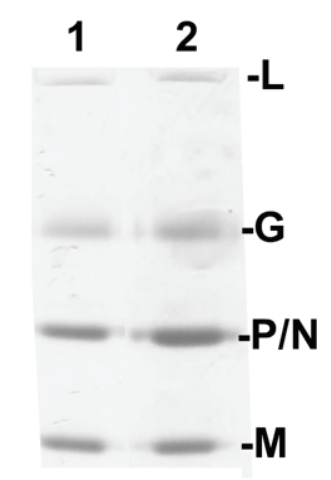

Figure 4-4. Characterization of rVSV with M loop mutations.

(A) One-step growth curve of rVSV M mutants and rVSV-wt in BHK-21 cells. The results show average titers at each time point from the experiment performed in triplicate.

The mutant rVSVs grow to one-log lower titer than Wt. (B) Coomassie-blue stained SDS-PAGE of $10^{7}$ PFU for rVSV-D125A (lane 1) and rVSV-wt (lane 2). (C) Transmission electron micrographs of purified rVSV-D125A (D125A) and rVSV-wt (Wt) virions. 130,000X, bar represents $20 \mathrm{~nm}$. 
A

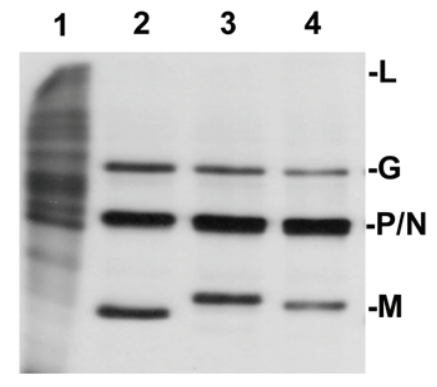

$6 \mathrm{hpi}$

B

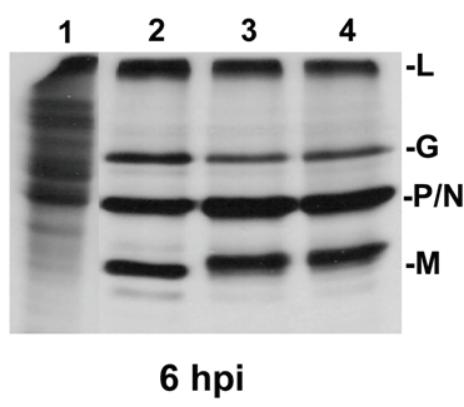

6 hpi

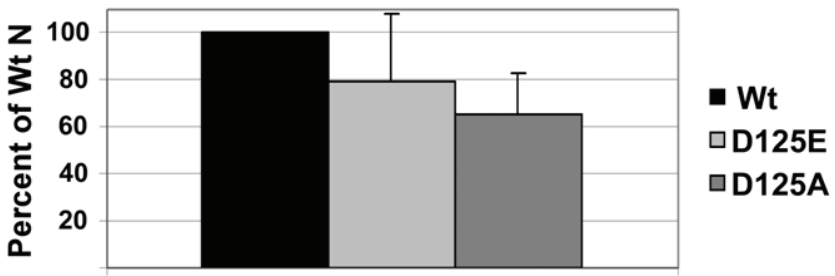

6 hpi

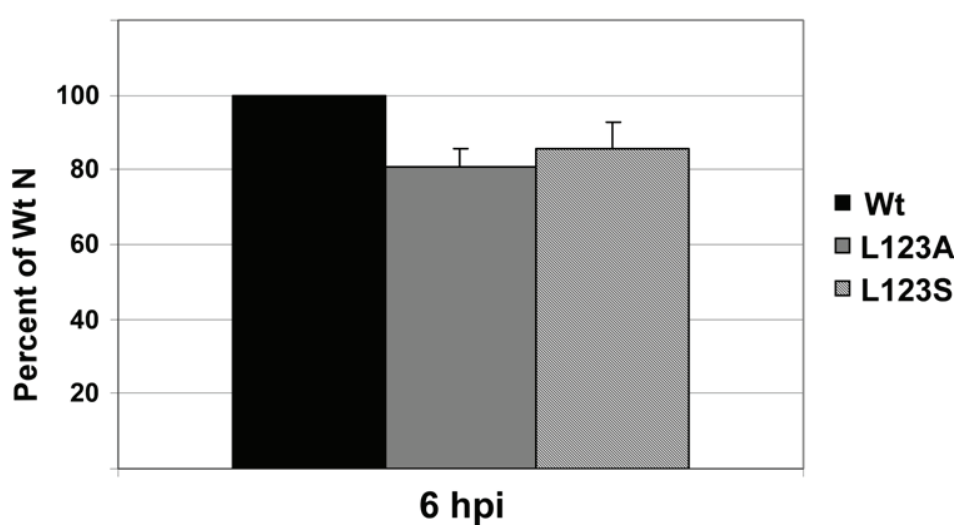

Figure 4-5. ${ }^{35} \mathrm{~S}$-methionine metabolic labeling of rVSV infected cells.

(A) SDS-PAGE fluorography of ${ }^{35}$ S-methionine pulse-labeled BHK-21 cell lysates from mock infected (lane 1), rVSV-wt (lane 2), rVSV-D125E (lane 3), or rVSV-D125A (lane 4) infected cells at 6 hours post infection (hpi). Quantification of the percent difference in labeled protein (mutant $\mathrm{N} / \mathrm{wt} \mathrm{N}$ protein) analyzed by phosphorimaging software is shown in the graph on the right. (B) SDS-PAGE fluorography of ${ }^{35}$ S-methionine pulsed BHK-21 cell lysates from mock infected (lane 1), rVSV-wt (lane 2), rVSV-L123A (lane 3 ), or rVSV-L125S (lane 4) infected cells at 6 hours post infection (hpi). Quantification of the difference in protein labeled (mutant $\mathrm{N} / \mathrm{wt} \mathrm{N}$ protein) analyzed by phosphorimaging software is shown in the graph on the right. 
more pronounced reduction in viral protein synthesis at $6 \mathrm{hpi}$ (Fig. 4-5). Note all recovered rVSV M loop mutants were analyzed in the following sections in triplicate but the rVSV-D125A mutant will be the focus in each of the remaining sections.

To examine the difference in protein synthesis between rVSV-wt and D125A infected cells in more detail, cells were pulse-labeled with ${ }^{35} \mathrm{~S}$-methionine for 10 minutes at 2, 4, 6, and 8 hpi and analyzed as described for Figure 4-4. Protein synthesis of viral and cellular proteins was similar for the two viruses at 2 and $4 \mathrm{hpi}$, with the characteristic inhibition of cellular protein synthesis seen from VSV infected cells beginning at 4 hpi. However, by 6 and 8 hpi there was a dramatic reduction in viral protein synthesis for the D125A mutant. Lane 1 shows cellular protein synthesis of mock infected cells. Quantification of viral protein synthesis at $8 \mathrm{hpi}$ from D125A infected cells showed an $82 \%$ reduction compared to wild-type levels (Fig. 4-6A (lane 8) and B). To determine if reduced viral protein synthesis could be observed outside of an rVSV infection, we transiently expressed the D125A or wild-type M proteins in cells and pulse-labeled the cells with ${ }^{35} \mathrm{~S}$-methionine at 18 hours post transfection (p.t.) and analyzed cell lysates immunoprecipitated with an M mAb by SDS-PAGE and phosphorimaging (Fig. 4-6C, lanes 1 and 2, respectively). We also analyzed protein expression using an in vitro transcription and translation system and compared the D125A mutant and wild-type by SDS-PAGE and phosphorimaging (Fig. 4-6D, lanes 1 and 2, respectively). Expression of D125A without other VSV proteins or products showed no reduced protein synthesis (Fig. 4-6C and D, lane 1) as observed at 8 hpi (Fig. 4-6A, lane 8) when compared to wildtype. These data suggested that the reduced viral protein synthesis observed is not solely due to the D125A mutant but requires other viral proteins or products in the context of infection.

To determine if the reduced viral protein synthesis was due to a reduction in viral mRNA, we performed quantitative real-time RT-PCR on RNA isolated from infected cells. Cells were infected with rVSV-wt or rVSV-D125A at an MOI of 10 and RNA extracts were prepared at increasing times post infection. The amount of genomic RNA was quantitated by RT-PCR using a VSV genomic standard (Fig. 4-7A), and when the ratio of $\mathrm{P}$ mRNA to genomic RNA was quantitated and we found the amount of P mRNA per genome was 3 fold higher at 5 hpi and 6 fold higher at 7 hpi in rVSV-D125A infected cells when compared to rVSV-wt infection (Fig 4-7B). These data showed that a decrease in viral mRNA synthesis was not responsible for the reduced viral protein synthesis seen with the D125A mutant. In fact, there was significantly more viral mRNA in the mutant infected cells than wild-type cells.

To find out if wild-type $\mathrm{M}$ could rescue the protein synthesis defect of the D125A mutant, we used a synchronized fusion assay in a co-infection experiment with rVSV-wt and rVSV-D125A. Cells were mock infected, infected with rVSV-wt (MOI 1), rVSVD125A (MOI 10), or rVSV-wt (MOI 1) and rVSV-D125A (MOI 10) in the presence of $\mathrm{NH}_{4} \mathrm{Cl}$ to synchronize the entry of virions (Chapter 2). This technique was used for the co-infection experiments because we have observed an increase in cells expressing both green fluorescent protein and red fluorescent protein from separate viral genomes in coinfected cells when fusion is synchronized as compared to normal (non-synchronized) 
Figure 4-6. Time course of viral protein synthesis by ${ }^{35} \mathrm{~S}-$ methionine metabolic labeling.

(A) SDS-PAGE fluorography of ${ }^{35} \mathrm{~S}$-methionine pulse-labeled BHK-21 cell lysates from mock infected (lane 1), rVSV-wt (lanes 3, 5, 7, and 9), or rVSV-D125A (lanes 2, 4, 6, and 8) infected cells at increasing time points post infection. (B) Quantification of the difference in protein labeled (mutant $\mathrm{N} / \mathrm{wt} \mathrm{N}$ protein) as analyzed by phosphorimaging software at 8 hours post infection shows an $80 \%$ loss in protein synthesis in rVSVD125A infected cells. (C) ${ }^{35} \mathrm{~S}$-methionine pulsed BHK-21 cell lysates from cells transiently expressing D125A (lane 1) or Mwt (lane 2). (D) ${ }^{35}$ S-methionine labeled protein from rabbit reticulolysates of an in vitro transcription and translation of D125A (lane 1) and Mwt (lane 2). 
A

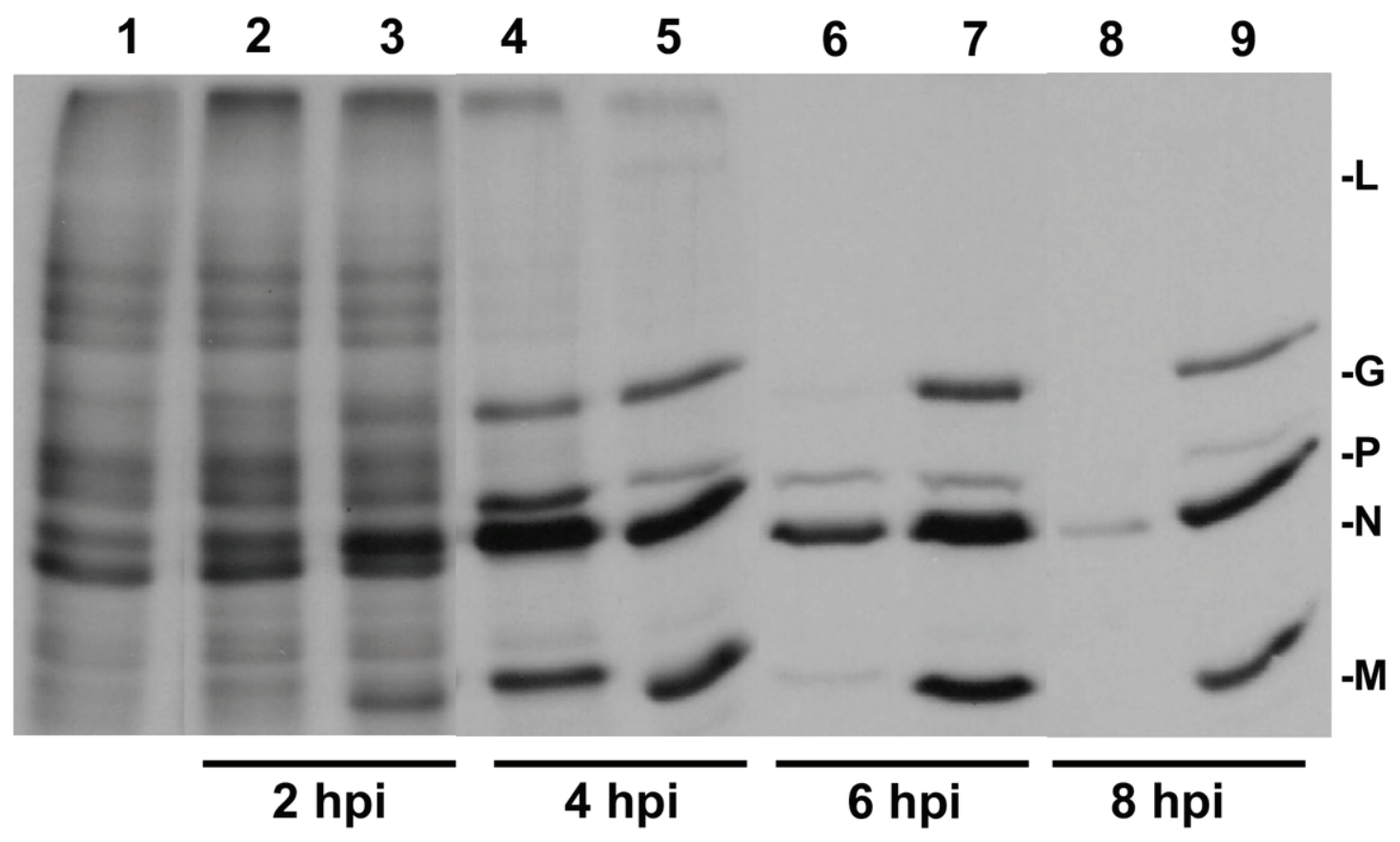

B
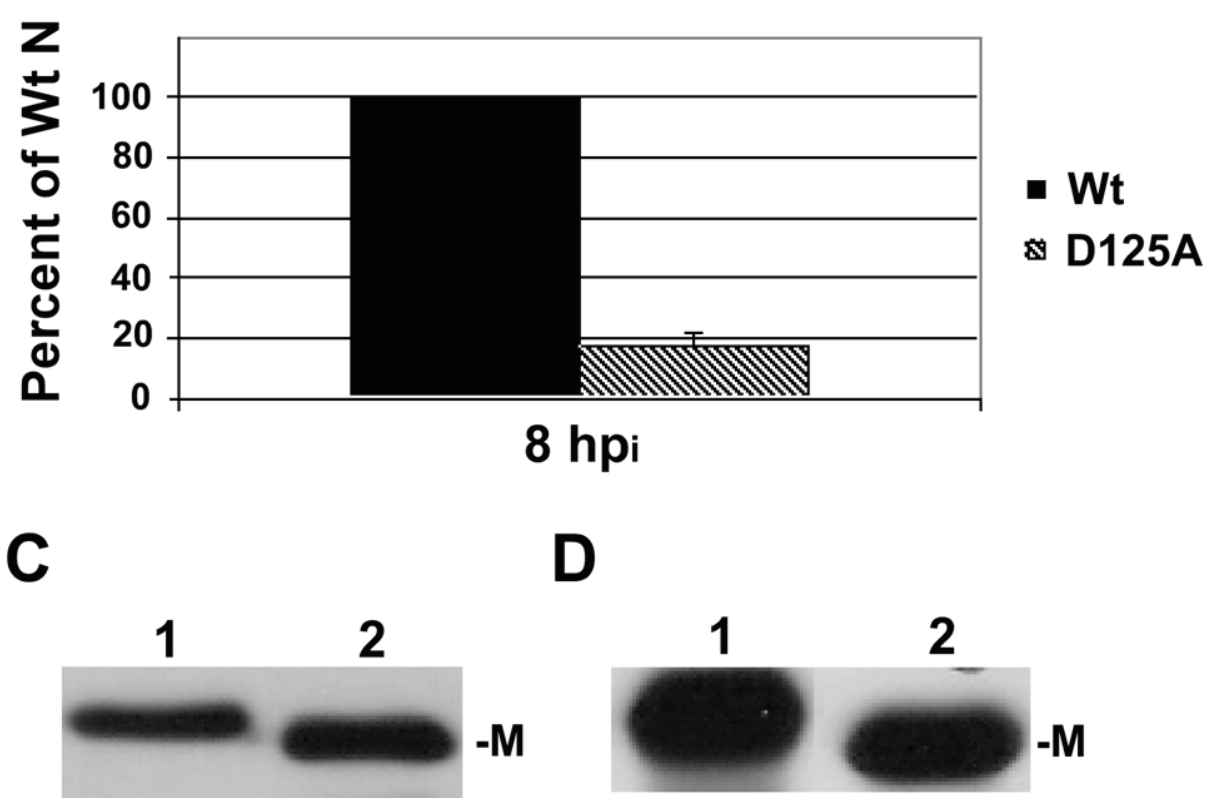


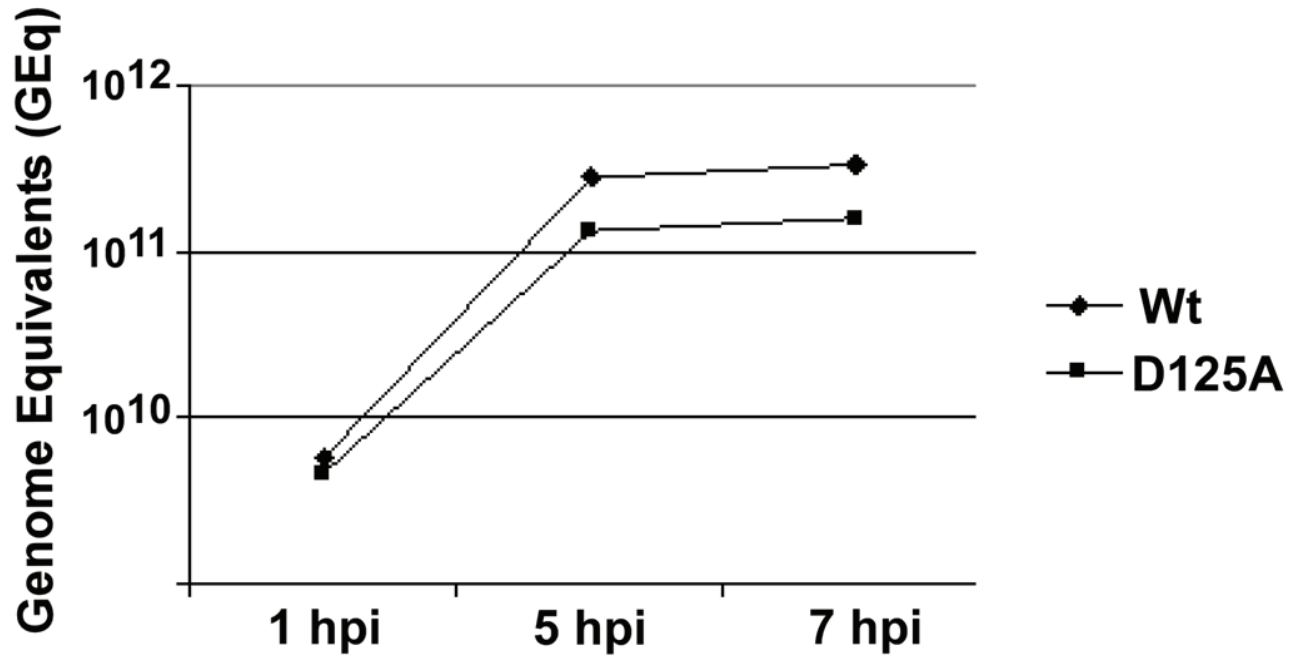

B

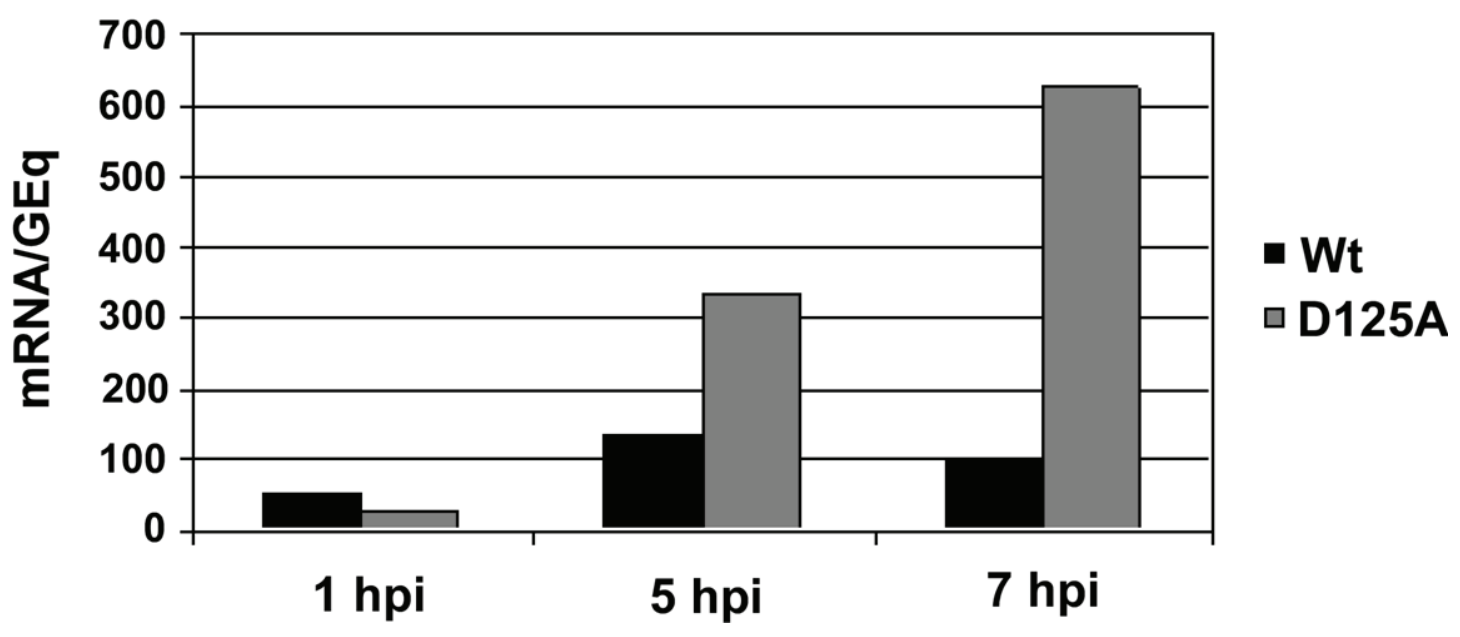

Figure 4-7. Quantitative real-time RT-PCR of virus genomes and mRNA during rVSV infection.

To rule out viral mRNA as the limiting factor in the reduction in protein synthesis at late time points during infection, RT-PCR was performed on BHK-21 cells infected with rVSV-wt (Wt) and rVSV-D125A (D125A). (A) Genome equivalents (based on purified VSV genomic RNA standards) of RNA extracted from cells at increasing time points post infection, measured by RT-PCR using SYBR green. (B) Levels of P mRNA were determined using quantitative RT-PCR and the $-\mathrm{Wt}$ and D125A mRNA to genome equivalents ratio was determined. rVSV-D125A infection leads to more mRNA per genome at 7 hours post infection showing that reduction in viral message did not effect protein translation. 
infection conditions (Watanabe, Mire, and Whitt, unpublished data). At 9 hpi cells were pulse-labeled with ${ }^{35}$ S-methionine and analyzed by SDS-PAGE and phosphorimaging (Fig. 4-8). We observed that co-infection could partially rescue protein synthesis at this time point post infection (Fig. 4-8A lane 3 compared to 1 ) and was up to $60 \%$ of rVSVwt infected cells (Fig. 4-8A lane 4 and Fig. 4-8B). These data showed that the D125A M protein is not a dominant negative mutant for the protein synthesis defect and could be partially rescued by the wild-type protein.

rVSV M loop mutants show defects in assembly. While we observed reduced viral protein translation beginning at $6 \mathrm{hpi}$, we also found that rVSV-D125A titers were one-log below wild-type (Fig. 4-4A) at 4 hpi when protein synthesis was similar between the two viruses (Fig. 4-6A, lanes 4 and 5). To determine if virus assembly was affected by the D125A mutation, we analyzed other assembly functions of M. It was previously observed that M protein associates with RNPs at the plasma membrane of VSV infected cells (157). To assess if this association was affected by the D125A mutation, we stained infected cells for $\mathrm{M}$ and $\mathrm{N}$ protein at 4 hpi and compared the distribution of the mutant and wild-type proteins by laser scanning confocal microscopy. The images in figure 49A are representative of infected cells stained with anti-M monoclonal antibody (23H12) conjugated to FITC and anti-N monoclonal antibody (10G4) conjugated to rhodamine and examined by $488 \mathrm{~nm}$ and $543 \mathrm{~nm}$ laser excitation. We observed similar distributions for the wild-type (Wt) and mutant (D125A) M proteins, but when we compared the $\mathrm{N}$ distributions in the D125A and wild-type (Wt) infected cells we found the $\mathrm{N}$ distribution during wild-type infection to be more closely associated with the cell periphery near the plasma membrane (Fig. 4-9A). These data suggested that RNPs were not being assembled at the plasma membrane as efficiently in rVSV-D125A infected cells when compared to rVSV-wt infection and we reasoned that this may affect the amount of virus released.

To determine if release of virions from infected cells was lower in rVSV-D125A infected cells when compared to rVSV-wt infected cells, we performed viral budding assays at 4 hpi. Cells were mock infected, infected with rVSV-wt, or rVSV-D125A at an MOI of 10 and at 3 hpi were pulse-labeled with ${ }^{35}$ S-methionine for 1 hour. At 4 hpi, virus was purified from the cell supernatants, cell lysates were collected, and both released virus and viral proteins in cell extracts were analyzed by SDS-PAGE and phosphorimaging. When the amount of labeled virus in the supernatant was compared to the cell lysates from rVSV-D125A it was found that only 1.3\% (Fig. 4-9B lanes 5 and 6) of labeled $\mathrm{N}$ protein was released from cells and rVSV-wt had 12\% (Fig. 4-9B lanes 3 and 4) labeled $\mathrm{N}$ protein released. We then determined whether this effect could be rescued by the wild-type $\mathrm{M}$ protein and we performed synchronized fusion co-infection experiments as mentioned above but in the context of the viral budding assay just explained. We observed that infection in the presence of $\mathrm{NH}_{4} \mathrm{Cl}$ did not affect virus release for rVSV-wt and rVSV-D125A at 4 hpi since the results were similar to that seen in Figure 4-9 with wild-type releasing 16\% (Fig. 4-9C lanes 1 and 2) of labeled $\mathrm{N}$ and the mutant releasing $0.9 \%$ (Fig. 4-9 lanes 3 and 4). However, we determined that coinfection of the virions resulted in partial rescue of virion release as labeled $\mathrm{N}$ protein 
A

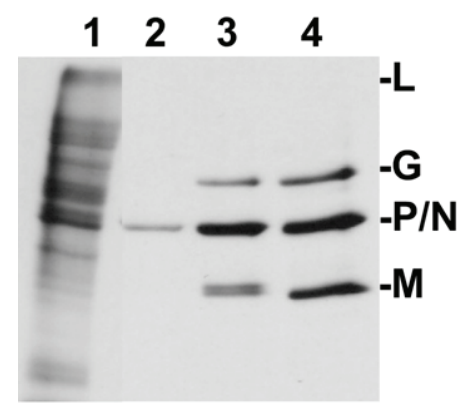

9 hpi
B

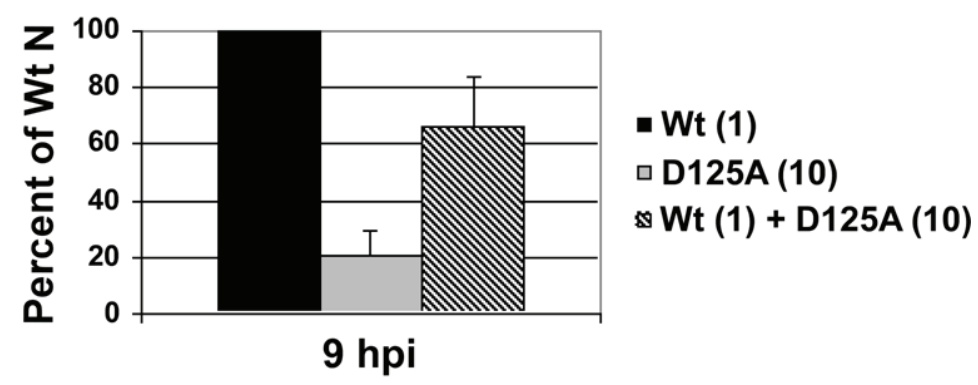

Figure 4-8. ${ }^{35}$ S-methionine metabolic labeling of cells co-infected with rVSV.

(A) Viral protein synthesis in BHK-21 cells was examined in mock infected (lane 1), infected with rVSV-wt (MOI 1, lane 4), rVSV-D125A (MOI 10, lane 2), or co-infected (lane 3) by ${ }^{35} \mathrm{~S}$-methionine pulse labeling at 9 hours post infection and cell lysates were analyzed by fluorography. (B) Quantification of protein synthesis using phosphorimaging shows a $60 \%$ rescue in co-infected cells. 
Figure 4-9. Analysis of assembly defects for rVSV-D125A.

(A) To characterize the distribution of the $\mathrm{N}$ and $\mathrm{M}$ proteins during assembly, laser scanning confocal microscopy of rVSV-wt (Wt) or rVSV-D125A infected cells (MOI 10) at 4 hours post infection was performed. Infected cells were fixed and labeled for the $M$ ( $\mathrm{M} \mathrm{mAb} 23 \mathrm{H} 12$ conjugated to FITC) and $\mathrm{N}$ ( $\mathrm{N} \mathrm{mAb} 10 \mathrm{G} 4$ conjugated to rhodamine) proteins. Magnification using 100X objective for a $1 \mu \mathrm{m}$ slice in the center of the cell. (B) Fluorography of cell lysates (odd numbered lanes) and released virus (even numbered lanes) from a viral budding assay of cells mock infected (lanes 1 and 2), infected with rVSV-wt (lanes 3 and 4), or rVSV-D125A (lanes 5 and 6) pulse-labeled with ${ }^{35} \mathrm{~S}$ -

methionine for an hour at 3 hours post infection (hpi). Phosphorimaging was used for the quantification of labeled $\mathrm{N}$ protein in the supernatant (released virus) versus cell lysates. (C) Co-infection data from viral budding assays as performed and quantified in (B). (D) RNP exchange assay. rVSV-wt (Wt RNP) or rVSV-D125A (D125A RNP) RNPs at 10, 50 , or $100 \mu \mathrm{g}$ were incubated with ${ }^{35} \mathrm{~S}$-methionine labeled Mwt or D125A produced by in vitro translation and the mixtures were ultracentrifuged over sucrose cushion to separate RNPs with associated M proteins incorporated input M (P; pellet) and un-incorporated $\mathrm{M}$ (S; supernatant). 
A

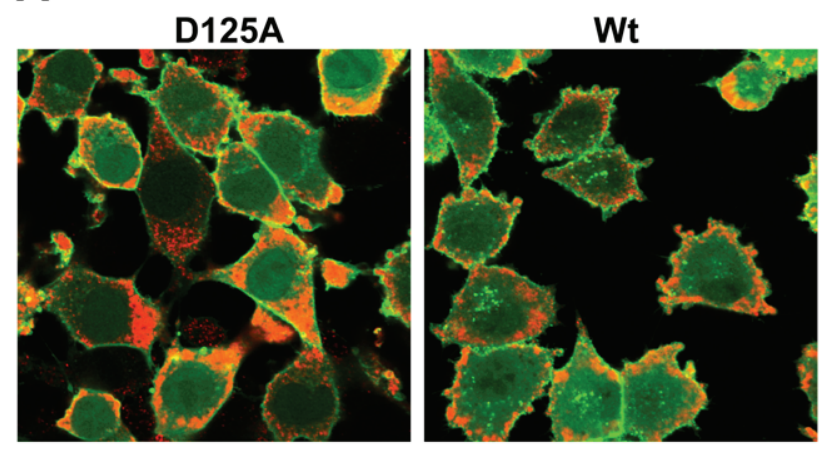

B

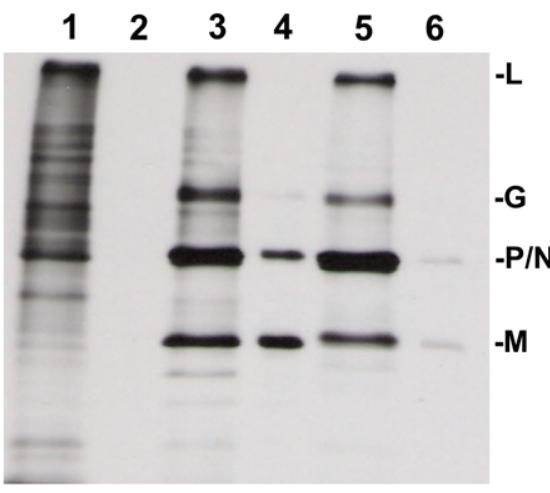

c

$4 \mathrm{hpi}$

4 hpi

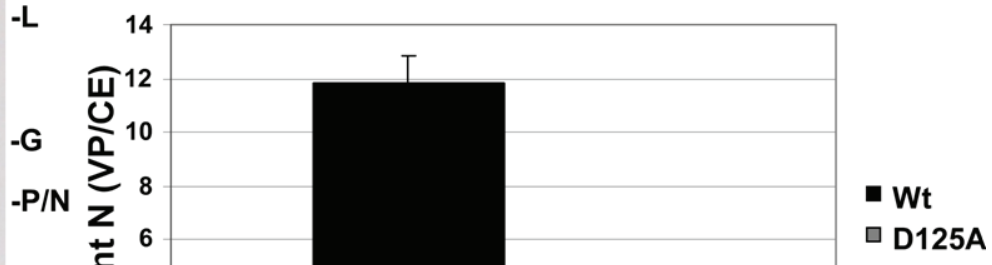

$\begin{array}{llllll}1 & 2 & 3 & 4 & 5 & 6\end{array}$

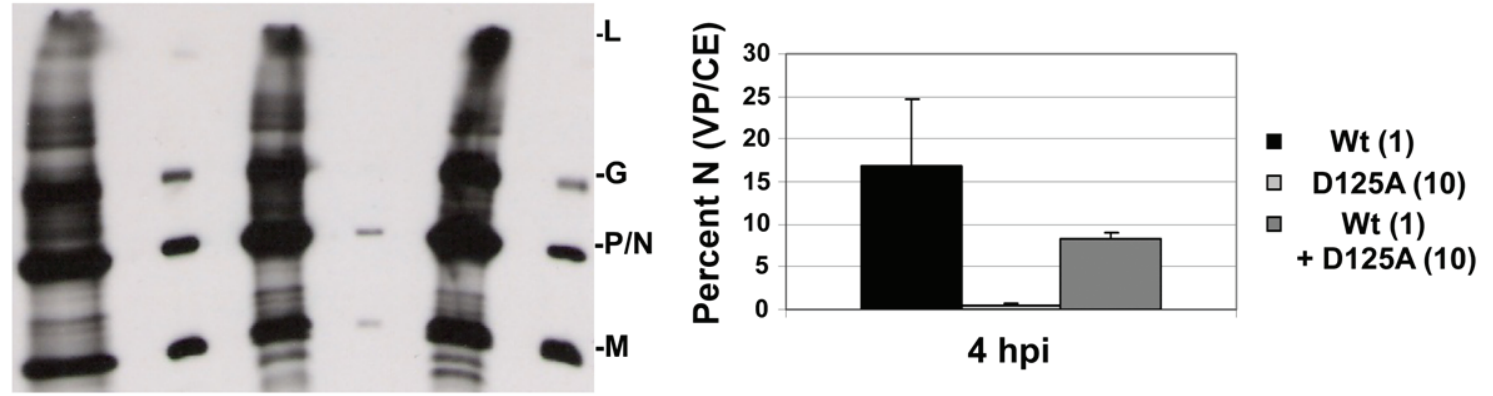

$4 \mathrm{hpi}$

D $10 \quad 50 \quad 100 \quad 10 \quad 50 \quad 100 \quad$-micrograms of Virus

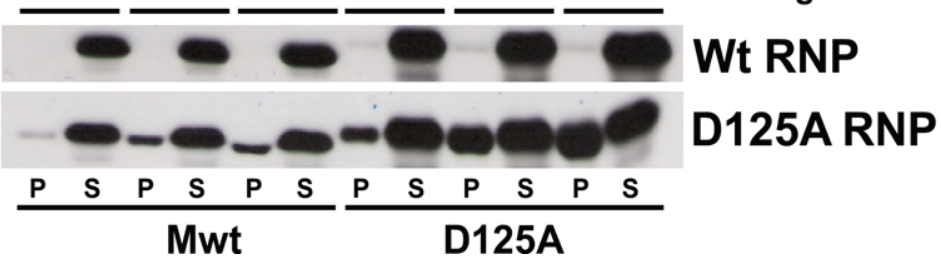


released was near 8\% (Fig. 4-9C lanes 5 and 6). These results suggested the D125A mutant $\mathrm{M}$ was not dominant negative for reduction virion assembly and release.

To determine if the decrease in virus release was due to inefficient condensation of RNPs by the D125A loop mutation, we performed an RNP exchange assay. Ten, 50, or $100 \mu \mathrm{g}$ of purified rVSV-wt or rVSV-D125A was detergent solubilized in the presence of ${ }^{35} \mathrm{~S}$-methionine labeled Mwt or D125A that had been synthesized in an in vitro translation system to allow exchange of the labeled M proteins with the RNPs of the virions. The RNPs were then separated from the supernatant by ultracentrifugation and the pellet and supernatant analyzed by SDS-PAGE and radiofluorography. We observed that the Wt RNPs did not incorporate any input ${ }^{35} \mathrm{~S}$-methionine labeled M protein even with $100 \mu \mathrm{g}$ of virus where more RNPs are available to exchange with input M (Fig. 49D, Wt RNP, P), while the D125A RNPs exchanged with each of the input labeled M proteins (Fig. 4-9D, D125A RNP, P). These data suggested that the D125A M protein was less tightly associated with the RNP derived from D125A virus and therefore resulted in exchange with input $\mathrm{M}$.

\section{Reduction in viral protein synthesis is not due to classical host responses,} apoptosis, or autophagy. To determine if the classical host responses to RNA viruses that can cause inhibition of viral and host protein synthesis were responsible for the reduced protein synthesis seen in rVSV-D125A infected cells, we examined the end products of RNase L and PKR activation. Cells were mock infected, infected with rVSVwt, or rVSV-D125A at an MOI of 10 and at increasing times post infection RNA was extracted for formaldehyde agarose electrophoresis (Fig. 4-9A) or cells lysates were separated by SDS-PAGE and analyzed by immunoblotting (Fig. 4-9B). To determine if RNase L was activated resulting in more degraded ribosomal RNA (rRNA) in the rVSVD125A infected cells, we examined extracted RNA on a RNA denaturing gel and observed that there were no degradation products from the $28 \mathrm{~S}$ and $18 \mathrm{~S}$ rRNA (Fig. 410A) where one would expect to see these products after RNase L activation. We then examined the cell lysates by immunoblotting for phosphorylated eIF2 $\alpha$ to determine if PKR activation was higher in cells infected with rVSV-D125A when compared to rVSVwt infection. We observed no increase in phosphorylation of eIF2 $\alpha$ in rVSV-wt and rVSV-D125A infected cells (Fig. 4-10B lanes 1-3, respectively). Taken together these data showed that neither classical response was involved in the decreased protein synthesis observed in D125A infected cells.

We used HeLa cells to analyze interferon, apoptosis, and autophagy responses to rVSV-D125A and rVSV-wt infections because the reagents required the use of human cells or only recognized human proteins. To determine if the reduced titers and protein synthesis phenotype of the D125A mutant were found in human cells we infected HeLa cells at an MOI of 10 and assessed total protein synthesis and virus production as described for the BHK-21 cell experiments. As seen in BHKs, protein synthesis was decreased for the rVSV-D125A (Fig. 4-11A lanes 6 and 8) when compared to rVSVwt (Fig. 4-11A lanes 5 and 7) but the reduction was somewhat delayed in HeLa and occurred at 6 hpi versus 4 hpi. The rVSV-D125A viruses also grew to lower titers in HeLa cells 
A

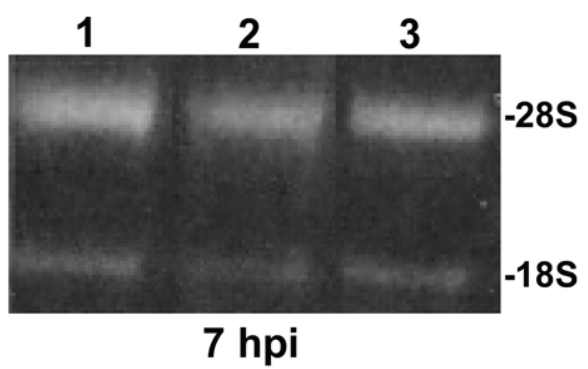

B

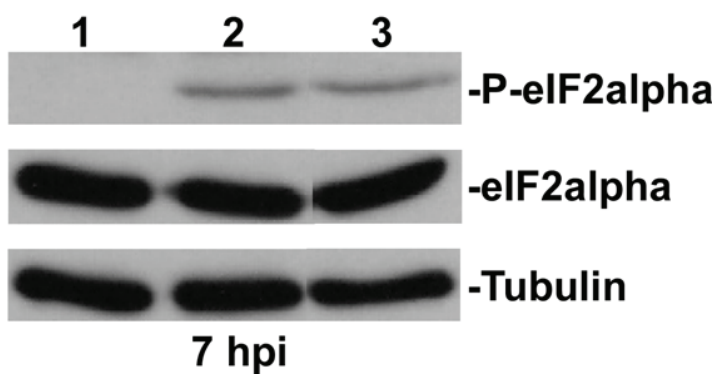

Figure 4-10. Analysis of PKR and RNaseL activation in rVSV-D125A infected cells. BHK-21 cells were mock infected (lane 1), infected at MOI 10 with rVSV-wt (lane 2) or rVSV-D125A (lane 3) and at 7 hours post infection cell extracts were collected and RNA was analyzed by denaturing gel electrophoresis (A) or by immunoblot analysis (B). (A) $2 \%$ formaldehyde-agarose gel of $2 \mu \mathrm{g}$ total input RNA to assess RNaseL activation. (B) Immunoblots using monoclonal antibodies to Tubulin, eIF2 $\alpha$, and phosphorylated eIF2 $\alpha$ was used to assess PKR activation. 
Figure 4-11. Analysis of reduced protein synthesis and host cell response in HeLa cells.

(A) HeLa cells were infected with rVSV-wt (lanes 1, 3, 5, and 7) or rVSV-D125A (lanes $2,4,6$, and 8) at an MOI of 10 and pulse-labeled with ${ }^{35}$ S-methionine at increasing times post infection. (B) rVSV-wt (Wt) and rVSV-D125A (D125A) titers at 8 hours post infection from single-cycle infection in HeLa cells. (C) HeLa cells transfected with a plasmid that expressed luciferase under the control of the IFN $\beta$ promoter were treated with $125 \mathrm{U}$ of human IFN $\beta$, or infected with rVSV-wt (Wt) or rVSV-D125A (D125A) and cell lysates were assessed for luciferase activity at 6 hours post infection. (D) Immunoblots of HeLa cell lysates from mock infected/untreated (lane 1) or rapamycin treated (lane 2) cells, and cell lysates from rVSV-wt (lanes 3, 5, and 7) or rVSV-D125A (lanes 4, 6, and 8) infected cells at increasing times post infection detecting phosphorylated eIF2 $\alpha$, full-length and cleaved caspase 3 , phosphorylated eIF4G, LC3B, VSV M, and tubulin using monoclonal antibodies. 
A
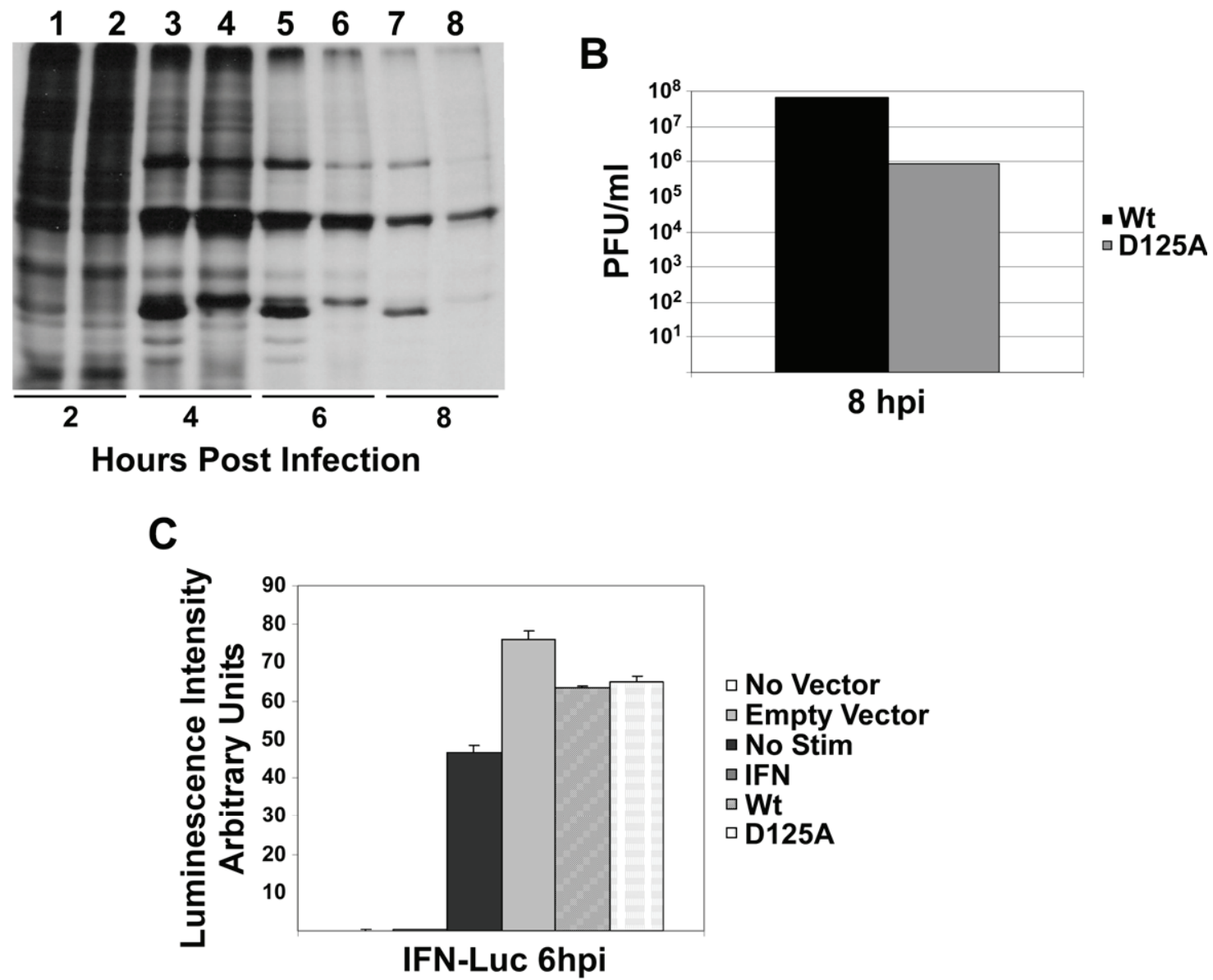

D

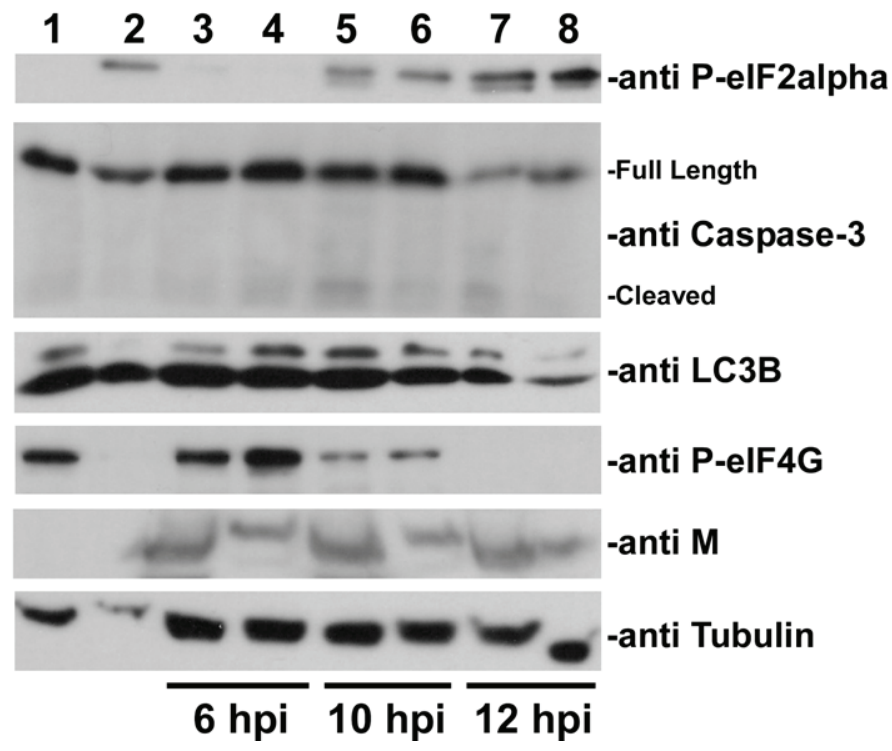


(also in NIH 3T3 and Vero cells; data not shown) when compared to rVSV-wt (Fig. 411B). These data showed the effects on protein synthesis and assembly were not restricted to BHK cells but also occurred in HeLa cells.

To determine if rVSV-D125A infection could lead to a more robust interferon response, we transfected cells with a luciferase reporter plasmid under the control of the interferon (IFN) $\beta$ promoter and at 5 hours post transfection the cells were treated with human (hu)IFN $\beta$, or they were infected with rVSV-D125A or rVSV-wt. At 6 hpi we observed no difference between the luciferase activity from the IFN $\beta$ promoter in cells infected with either virus (Fig. 4-11C). These data showed that in these cells increased IFN inducible gene expression is not responsible for the reduction in rVSV-D125A infected cells.

To determine if eIF2 $\alpha$ phosphorylation, apoptosis, or autophagy could cause reduced protein synthesis in rVSV-D125A infected HeLa cells when compared to rVSVwt infection, cells were mock infected or treated with rapamycin to induce autophagy as a control, and were infected (MOI 10). Cell lysates were collected at 6, 10, and $12 \mathrm{hpi}$ and analyzed by immunoblot. Phosphorylation of eIF2 $\alpha$ was enhanced in rVSV-D125A infected cells at 12 hpi but not at 6 hpi (Fig.4-11D lanes 8 and 6, respectively) where we observed reduced viral protein synthesis suggesting PKR activation is not involved in this effect seen at earlier times post infection. Apoptosis was ruled out as a possible mechanism for this effect as there was no difference in the cleavage of caspase 3 between rVSV-D125A and rVSV-wt infected cells (Fig. 4-11D, lanes 3-8). To examine the role of autophagy in reduced viral protein synthesis we examined proteins affected by this process and compared them between the two types of virus infected cells. Rapamycin treatment resulted in the complete conversion of LC3 protein to the LC3B form and the dephosphorylation of eIF4G, which is an important protein in the initiation of protein synthesis (Fig. 4-11D lane 2). However, we observed no difference between these autophagy markers within cells infected with rVSV-D125A or rVSV-wt. Overall, these data showed that PKR activation, induction of apoptosis, and autophagy were not involved in the reduction in viral protein synthesis seen in rVSV-D125A infected cells.

rVSV infection does not reduce cap-independent protein synthesis. To determine if protein synthesis from a Type 2 internal ribosome entry site (IRES) could be affected in rVSV-D125A infected cells, we transfected BSR cells constitutively expressing $\mathrm{T} 7$ polymerase with a plasmid that has a T7 promoter used to produce an mRNA with an encephalomyocarditis virus (EMCV) IRES for cap-independent protein synthesis of a luciferase reporter gene. At 18 hours p.t., cells were mock infected, treated with cycloheximide, or infected with rVSV-wt or rVSV-D125A at an MOI of 10. Cell lysates were then analyzed for luciferase activity in a luminometer at increasing times post infection. Luciferase activity of cell lysates from mock infected and cycloheximide treated BSRs at 6 hpi (of treatment) showed the basal level and background luciferase activity, respectively (Fig. 4-12). It is interesting to note that at $6 \mathrm{hpi}$ both rVSV-wt and rVSV-D125A infected cell lysates have higher luciferase activity than the mock infected cells. This effect may be due to restriction of host mRNA export from the nucleus which 


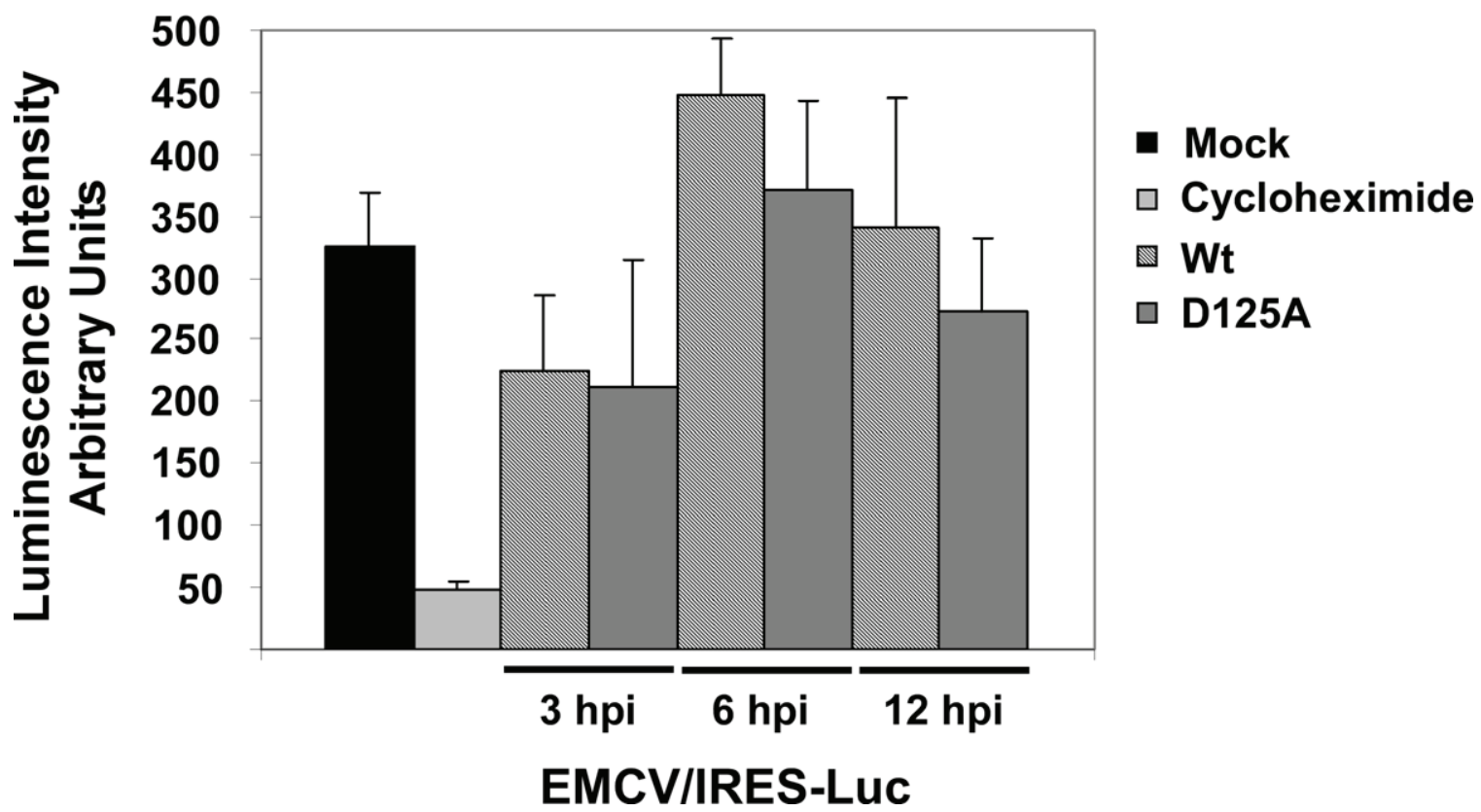

Figure 4-12. EMCV/IRES expression of luciferase in rVSV infected BSR cells. BSR cells constitutively expressing $\mathrm{T} 7$ were transfected with a plasmid that expresses IRES-luciferase from a T7 promoter. Eighteen hours post transfection; BSR cells were mock treated/infected or treated with cycloheximide, or infected rVSV-wt (Wt) or rVSVD125A (D125A). Luciferase activity was measured at 6 hours post treatment for cycloheximide treated cells and at increasing times post infection for infected cells. 
would reduce the competition for ribosomes by the cytosolic luciferase mRNA since in another experiment we found that both viruses were capable of limiting luciferase expression from a chicken actin promoter, which occurs in the nucleus by RNA polymerase II (data not shown). The results of this experiment also showed that the luciferase activity of cell lysates from both viruses did not significantly differ over the course of infection suggesting that the reduced protein synthesis seen at increasing time points post infection in rVSV-D125A infected cells only affected synthesis of proteins produced by a cap-dependent mechanism.

\section{Discussion}

The crystallization of the VSV M protein was made possible by proteolytic cleavage of an exposed loop that prevented the protein's self-association (10, 65-68). The self-association is affected by high ionic concentrations (67) and these salt conditions have also been shown to cause the decondensation of skeletons in an M-dependent manner (154). Altogether, these observations suggested that the exposed loop may be involved in the assembly of virions in regards to condensation of RNPs into skeletons. Others made mutations at amino acids 121 to 124 within this loop and a minor assembly defect was observed but there was an unexpected reduction in viral protein synthesis for this mutant rVSV (35). Here we report on a mutational analysis of different amino acids found within this loop with a focus on the conserved aspartate at residue 125 .

There were no discernable differences in cellular distribution and budding activity for the M loop mutants when compared to Mwt. However, rVSV encoding only four of the eight mutations could be recovered after multiple attempts ( $n=6$ per virus). These data suggested that the amino acids and size of the loop were important for recovery and therefore for assembly of virus. Indeed, we saw reduced growth kinetics (Fig. 4-4A) and virus budding (Fig. 4-9B) for the four rVSV M loop mutants that were recovered when compared to wild-type rVSV. We also observed what appeared to be a build-up of $\mathrm{N}$ protein (RNPs) within rVSV-D125A infected cells when compared to rVSV-wt (Fig. 49A) and we reasoned that this may be due to inefficient condensation of RNPs at the plasma membrane much like the build-up of uncondensed RNPs that occurs in the M protein temperature-sensitive mutant (tsG33) infected cells at non-permissive temperature (160). There were also additional observations that suggested RNPs may be inefficiently condensed. One was the high viral mRNA to genome ratio found in the rVSV-D125A infected cells when compared to rVSV-wt infection as M condensation of the RNP is thought to inhibit transcription (163). The other was that rVSV-D125A RNPs could exchange with more Mwt or the M D125A loop mutant proteins when compared to rVSV-wt RNPs (Fig. 4-9D) where exchange of M protein is thought to be indicative of the condensed nature of RNPs (57). These data correlated with the proteolytic cleavage data that suggested the exposed loop may be important in virion assembly $(65,66)$. These were not the only observations that we made in regards to the M loop mutations as we also noticed a reduction in viral protein synthesis (Fig. 4-5 and 4-6A). 
The observation that the rVSV-D125A mutant had a reduction in protein synthesis was also observed previously with different $M$ loop mutations (35). The assembly defects and higher viral mRNA to genome ratio phenotypes of the rVSVD125A mutant led us to reason that there may be more viral products within infected cells to provide a more robust antiviral response to the D125A mutant when compared to wild-type. To test the classical antiviral responses of cells to RNA virus infection that result in reduced protein synthesis, we compared PKR and RNaseL activation (196) in cells infected with rVSV-D125A and rVSV-wt for differences in phosphorylation of eIF $2 \alpha$ and degradation of rRNA and found these were not responsible for the reduction (Fig. 4-10). The expression IFN which leads to subsequent signaling events that produce and antiviral response that controls VSV replication (197), was also we examined by luciferase expression from an IFN $\beta$ promoter and it was found that there was no difference in the induction of IFN between the rVSV-wt and D125A mutant viruses, suggesting this was not responsible for the reduced viral protein synthesis (Fig. 4-11C). The experiment examining activity from the IFN $\beta$ promoter was performed in HeLa cells, but another cell line with a more robust IFN response like plasmacytoid dentritic cells (pDCs) may be a more appropriate cell to infect and to evaluate potential differences before completely disregarding the role of this pathway.

VSV can cause apoptosis in infected cells (106) but enhanced apoptosis did not appear to be involved in this reduction as there was no difference in cleavage of proCaspase 3 to caspase 3 when loop mutant and wild-type infected cells were compared (Fig. 4-11D). Recently, it was reported that the IFN response to VSV infection in pDCs was toll-like receptor 7 (TLR7) and autophagy dependent (118). This revealed a new model for how cells can autophagocytose viral ssRNA and traffic the RNA to TLR7. We therefore measured this response by examining the conversion of LC3 to LC3B in infected cells and found no difference between the virus infected cells. Altogether, these results suggest that the reduced viral protein synthesis may not be a host cell response, our analysis was not exhaustive as other responses like those through the cytosolic pattern recognition receptors such as RIG-I could be involved (187).

Recently, the VSV field has gained insight on how control of the translation of host and viral proteins is accomplished in VSV infected cells. It was shown that dephosphorylation of the eIF4E complex was responsible for the reduction of host protein synthesis (34) and phosphorylation of eIF $2 \alpha$ controls viral protein synthesis at later times post infection (33). A new model has been proposed for translation during infection where dephosphorylation of eIF4E results in inhibition of host protein synthesis and the host mRNA is then shuttled to inactive mRNPs which in turn allows for newly made viral mRNA to be translated (208) and this process may be enhanced between 4 to 8 hpi by M $(13,35,208)$. This is particularly interesting since we observed a reduction in viral protein synthesis from 4 to $8 \mathrm{hpi}$ (Fig. 4-6A), which we assumed was the result of a host response since protein synthesis was similar at early times post infection. However this may reflect the inability of the D125A loop mutant to positively regulate viral protein synthesis. 
Eukaryotic protein translation initiation is a complex process that requires many co-factors but the cap-independent translation of mRNA from picornaviruses such as encephalomyocarditis virus (EMCV) requires a smaller number of initiation factors when compared to the number required for cap-dependent translation initiation (167). EMCV mRNA contains a 5' untranslated region (UTR) that has an internal ribosome entry site (IRES) $(83,89)$ and translation from the IRES in vitro only requires ATP, eIF2, eIF3, eIF4A or eIF4E, and the central portion of eIF4G (167). Although it is still possible that the reduction in viral protein synthesis was due to a host response that was enhanced due to inefficient virus assembly, our results suggested that the effect was more likely due to the inability of the loop mutant proteins to up-regulate VSV translation. To determine if any of the factors required for translation from the EMCV IRES were affected during rVSV-D125A infection, we examined translation of luciferase expressed from an EMCV IRES and found it was not significantly affected by rVSV-D125A infection.

Interestingly, there was an increase in luciferase at $6 \mathrm{hpi}$ in infected cells when compared to mock infected cells (Fig. 4-12) where newly made viral mRNA should be preferentially translated (208). These data implicate that eIF4E may be the factor involved in the protein synthesis effect observed during rVSV M loop mutant infections (35) since it is the only major initiation factor that is not required for EMCV IRES protein translation initiation but is required for cap-dependent $\beta$-globin mRNA translation (167). Importantly, it has been shown that VSV infection affects host protein synthesis by dephosphorylation of eIF4E (34) and a model was proposed that the short UTR of VSV mRNA did not require phosphorylated eIF4E for translation initiation (33) and therefore viral protein synthesis was not affected. The short UTR model was tested by expression of protein from rVSV genomes that had long UTRs on the mRNA and it was observed that the mRNA with long UTRs were translated as efficiently as mRNA with shorter UTRs (209). This led to the model suggesting that it was the timing of the mRNA synthesis that allowed for preferential translation (208). The data presented here together with that published by others $(13,33-35,208,209)$ suggests that M protein may regulate viral translation by playing a supporting role for the loss of phosphorylated eIF4E and that the exposed loop of $\mathrm{M}$ (amino acids 121 to 125) is involved in this regulation to allow cap-dependent translation of newly synthesized mRNA. However, not only may this loop be involved in protein translation enhancement, but also we show that it is required for efficient assembly. Whether these two phenotypes can be separated genetically will require additional investigation. 


\section{Chapter 5: General Discussion}

\section{VSV Entry and Uncoating}

Entry and uncoating of enveloped viruses has been examined for the past three decades but new technologies over the past 10 years have made it possible to gain further insight into these processes [reviewed in $(114,136)]$. Virions entering a cell have highly condensed genomes protected by proteins. Before these particles can replicate in a host cell, after fusion and entry, uncoating must occur and this is described as the dissociation of the proteins condensing the genome. Once the genome has uncoated, replication of the virus can begin and this process is the opposite of what occurs to package the virus for release. Here lies the question; how can a virus accomplish these two distinct events using the same protein? It has been shown how influenza virus, another enveloped negative-strand RNA virus, can accomplish this through virus-host interactions. Studies on Sindbis virus and Semliki Forest virus have also suggested virus-host interactions in uncoating. Based on the available details for VSV entry and influenza, Sindbis, and Semliki Forest virus entry and uncoating, I am proposing a model for VSV entry and uncoating with a focus on the events that occur right before fusion and after release into the cytosol.

Upon entry, the VSV virion must be disassembled. The structure of the virion is composed of a helical nucleocapsid, containing L-P-N-RNA-M, surrounded by a membrane derived from an infected host cell membrane with transmembrane, trimeric $\mathrm{G}$ proteins protruding from the surface. The RNA genome is encapsidated by $\mathrm{N}$ protein and RNA-dependent RNA polymerase, consisting of the $\mathrm{L}$ and $\mathrm{P}$ proteins, is associated with this ribonucleoprotein complex (199). The L-P-N-RNA complex makes up the ribonucleoprotein particle (RNP) which is coiled into a tightly packed helix and the M protein condenses the RNP into this tightly packed helix, called the skeleton $(153,154)$. Also a population of membrane bound $\mathrm{M}$ anchors the skeletons to the viral membrane $(31,151)$. Viral uncoating, in the case of VSV, is regarded as the dissociation of M from RNPs and is an essential step in entry (130). Without the release of M protein transcription of viral mRNAs (productive infection) cannot proceed since it has been shown that M protein inhibits transcription $(32,44,212)$.

VSV entry. When VSV enters the host cell through the clathrin-mediated endocytic pathway (139), it uses the decreasing $\mathrm{pH}$ of endocytic organelles as a trigger for fusion in order to penetrate the cell as is the case for influenza virus (207). In our model, VSV virus attaches to the surface of a cell and is endocytosed by coated vesicles (Fig. 5-1A) and transported through the endosomal pathway to early endosomes (Fig. 51B). In early endosomes (189), $\mathrm{pH}$-dependent fusion of the endosomal and viral membrane occurs (Fig. 5-1C) and the G protein is directly responsible for these entry steps $(18,82,134,161,198,211)$. Our model presents early endosomes as the location 
Figure 5-1. VSV entry model.

(A) Attachment to the cell surface by VSV. (B) Subsequent clathrin-mediated endocytosis of a VSV particle in a coated pit (orange stars depict clathrin scaffolding) that leads to Rab5 dependent trafficking to the early endosomes where a $\mathrm{pH}$-dependent conformational change occurs for $\mathrm{G}(\mathrm{C})$ resulting in acidification of the virion interior (D). Acidification of interior of the virion has an effect on the $\mathrm{M}$ protein (E, bluebordered stars) and results in an enhanced dissociation of $\mathrm{M}$ after fusion (F). The bulk of $\mathrm{M}$ remains associated with endosomes and is trafficked to the recycling endosomes where $\mathrm{M}$ is thought to be located at the point of fusion $(\mathrm{G})$. A smaller fraction of $\mathrm{M}$ is released into the cytosol and reaches the NPCs in a microtubule and actin independent manner $(\mathrm{H})$. Events observed in steps $\mathrm{G}$ and $\mathrm{H}$ correlate with replication of RNPs that have uncoated. 


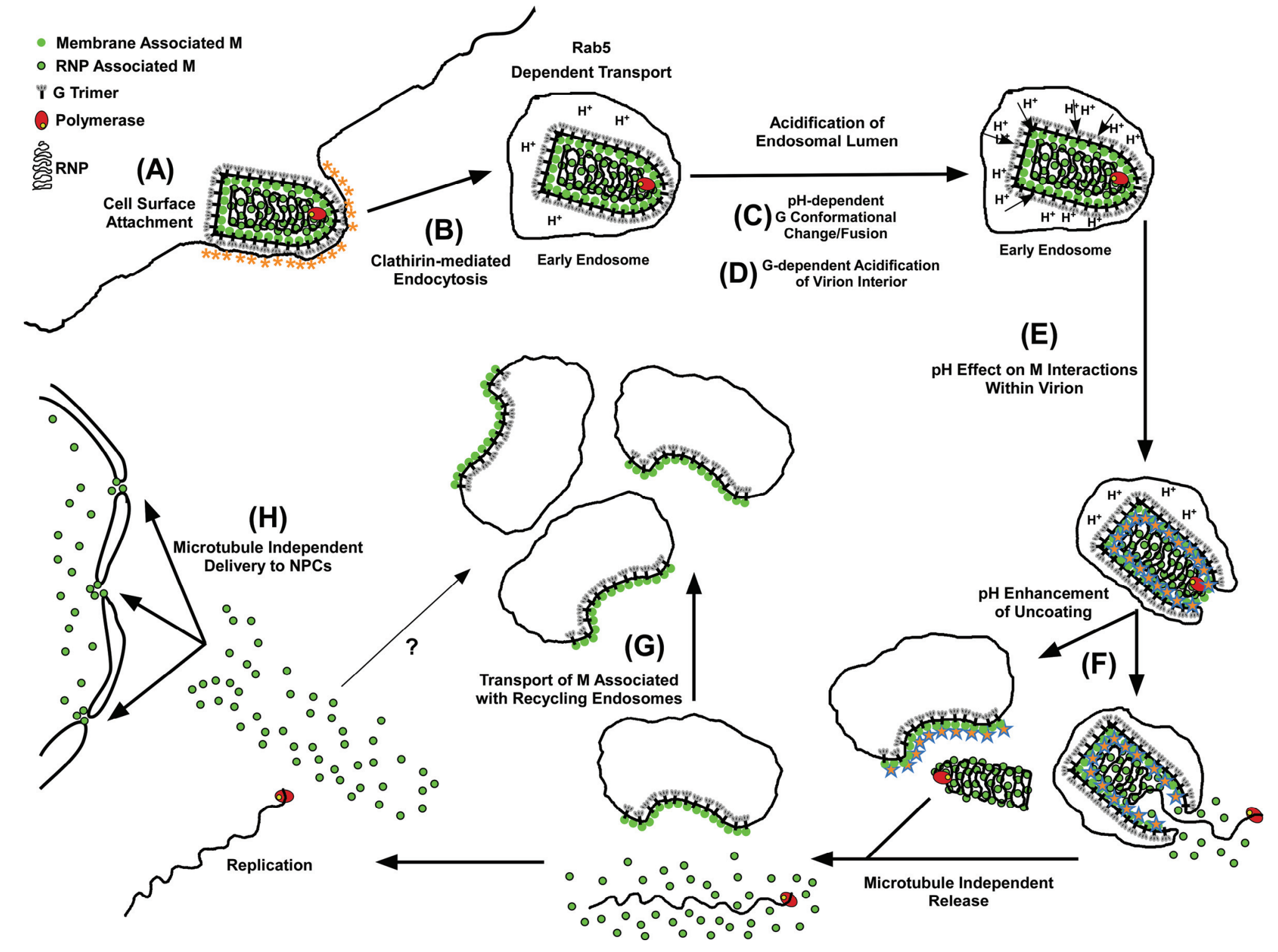


of fusion and release of RNPs based on Rab5 dominant negative (189) and nocodazole (42) studies that indicate fusion does not require transport to late endosomes as another group has recently suggested (117).

Acidification of the VSV interior and its effect on M. Our model proposes that the interior of VSV becomes acidified over the course of entry in a G-dependent manner (Fig. 5-1D). This is a different mechanism than the one used for influenza as discussed in Chapter 3 (Discussion section) but results in a similar enhancement of the uncoating of the $M$ protein. Since the M associated with purified RNPs is not sensitive to acidic conditions [(130), and our lab's unpublished data] our model has the $\mathrm{pH}$ affecting the interaction between the membrane bound and RNP bound M (Fig. 5-1E, blue-bordered stars) which allows the release of RNPs from endosomes after fusion. The release of RNPs could be a general unwinding of the RNP from one end or release of the condensed RNP as depicted in figure $(5-1 \mathrm{~F})$.

Completion of uncoating and the subsequent distribution of $M$. In our model, after the release of the skeleton from the membrane bound $\mathrm{M}$, the bulk of the M protein remains associated with endosomes that traffic to the recycling endosomes (Fig. 5-1G). Although binding to one side of the endosome by the $\mathrm{G}$ of unfused virions or the reassociation of released $\mathrm{M}$ protein to endosomes cannot be completely ruled out, the separation of M and the RNPs at these locations and the fact that only misfolded temperature-sensitive $\mathrm{M}$ mutants have been seen to associate with vesicles in cells (132) suggests that these are points of fusion. The RNP associated $\mathrm{M}$ then releases from the RNPs into the cytosol as previously proposed (130) and reaches the nucleus and binds the NPCs by diffusion (Fig. 5-1H). Diffusion of M to the NPCs is proposed here since the protein could reach the nucleus and separate from RNPs without the need for actin or microtubules; however an undiscovered mode of trafficking perhaps using RaeI or vesicular transport that is not dependent on these two cytoskeletal elements cannot be ruled out. Brefeldin A inhibition of retrograde transport could rule out that transport process in the trafficking to NPCs (27). siRNA knock down or use of dominant negatives of the known Rab GTPases involved in vesicular trafficking may also be ways to rule out known vesicular pathways that are commandeered by bacterial pathogens to survive within host cells (23). The binding of M to NPCs has been proposed previously as the first cytopathic effect of VSV (60) and one could reason that directly after release of M the association with the NPCs would give the virus the ability to prevent antiviral responses requiring gene expression before the virus has started replication.

\section{VSV Assembly}

The assembly of VSV virions, which is the reverse process of uncoating, has been studied extensively and a model has been proposed by our lab (90). Proteolytic cleavage of an exposed loop prevented M self-association $(10,65-68)$ and this self-association has been implicated in condensation of skeletons (154). These observations suggested that 
the exposed loop may be involved in the assembly of virions in regards to condensation of RNPs into skeletons. Others made mutations at amino acids 121 to 124 within this loop and a minor assembly defect was observed, but there was an unexpected reduction in viral protein synthesis for this mutant rVSV (35).

The current model from Jayakar, et al. (90), outlines 5 steps in VSV assembly. The first step is encapsidation of anti- or genomic RNA by the N protein in the cytoplasm while some $\mathrm{M}$ may bind to the nucleocapsid but not enough for condensation. The second step occurs during $\mathrm{N}$ encapsidation of genomic RNA, where G protein is trafficked to microdomains at the cell surface forming favorable sites for condensation of RNPs by M protein. The third step consists of $\mathrm{M}$ association at the plasma membrane (during steps 1 and 2) and condensation of RNPs into skeletons with cytosolic M protein at G-enriched microdomains. At the fourth step, the condensation of skeletons resulting in eversion of the the plasma membrane resulting in an enveloped skeleton by recruitment of membrane and cytosolic M proteins, resulting in bullet shaped evaginations of the membrane. In the final step of the model, the host cell multivesicular body (MVB) fission machinery associates with the PY motif of M through a ubiquitin ligase and this interaction, whether direct or indirect, results in fission and release of mature progeny virions.

Based on the assembly model from our lab, I propose a model for the involvement of the exposed loop in efficient binding to and condensation of RNPs by M during VSV assembly. Initiator $\mathrm{M}\left(\mathrm{M}_{i}\right)$ has been proposed and is yet to be identified however nucleation sites are needed for full scale M self-association to occur (67) and one can imagine a scenario where $\mathrm{M}_{i}$ can bind to RNPs before they reach the plasma membrane to prime the RNPs for condensation by M protein undergoing self-association. Also M inhibits viral transcription within infected cells and this does not necessarily occur at the plasma membrane so $\mathrm{M}_{i}$ could be responsible for the phenomenon. The cleavage of the exposed loop of $\mathrm{M}$ by thermolysin resulted in $\mathrm{M}_{\mathrm{th}}$ which could not form, nor be recruited into nucleation sites (65). I propose a model where the conserved aspartate at residue 125 is involved in the process of initial binding of $\mathrm{M}_{i}$ to RNPs (Fig. 5-2, between I. and II.) and the efficient condensation of RNPs at the plasma membrane (Fig. 5-2, V.) as evidenced by the build up of N protein, hyper-transcription of viral mRNA in rVSVD125A infected cells and the increased ability of the RNPs from virions to exchange with introduced M. This model also includes the most recent model for how budding sites are formed, where RNPs and G initially associate at microdomains before incorporation of M at these sites (195) (Fig. 5-2, III.). The importance of the hypervariable hinge region of $\mathrm{P}$ in assembly (43) may be the important factor in the initiation of association of RNPs at budding sites or for condensation by M (Fig. 5-2, III. and IV., respectively). Also observations from Chapter 2 revealed $\mathrm{M}$ chimeras with large C-terminal fusions affect budding activity suggesting that the PY motif interactions with Nedd4 or an MVB complex are sterically hindered by these $\mathrm{C}$-terminal extensions thereby reducing the budding activity of M protein (Fig. 5-2, VI.). 
Figure 5-2. VSV assembly model.

(I.) Genomic RNA is encapsidated by N protein while $\mathrm{G}$ protein trimers form microdomains at the virus budding site (II.). Initiator $\left(\mathrm{M}_{i}\right.$, orange circles) has been proposed previously (90) and as described in the General Discussion the LXD sequence of the $\mathrm{M}$ protein may be important for forming nucleation sites formed by the $\mathrm{M}_{i}$. After the proposed binding of $\mathrm{M}_{i}$ to the RNP this complex is then trafficked to the plasma membrane where it associates in microdomains with G (III.) and then M (membrane bound green circles) is incorporated to these microdomains (IV.) as recently shown (195). The role for $\mathrm{P}$, in particular residues 191 to 200 , in assembly has been reported recently and it may be involved in steps III. or IV. (V.) After the budding site has been formed the membrane bound $\mathrm{M}, \mathrm{M}_{i}$, and cytosolic $\mathrm{M}$ (black-rimmed green circles) participate in the helical condensation of the RNP and this process is proposed to be less efficient in M proteins with mutations in the LXD sequence. (VI.) Progeny virions then bud from the host cell in an M protein PY motif dependent manner where it has also been shown that large $\mathrm{C}$-terminal extensions affect budding. 

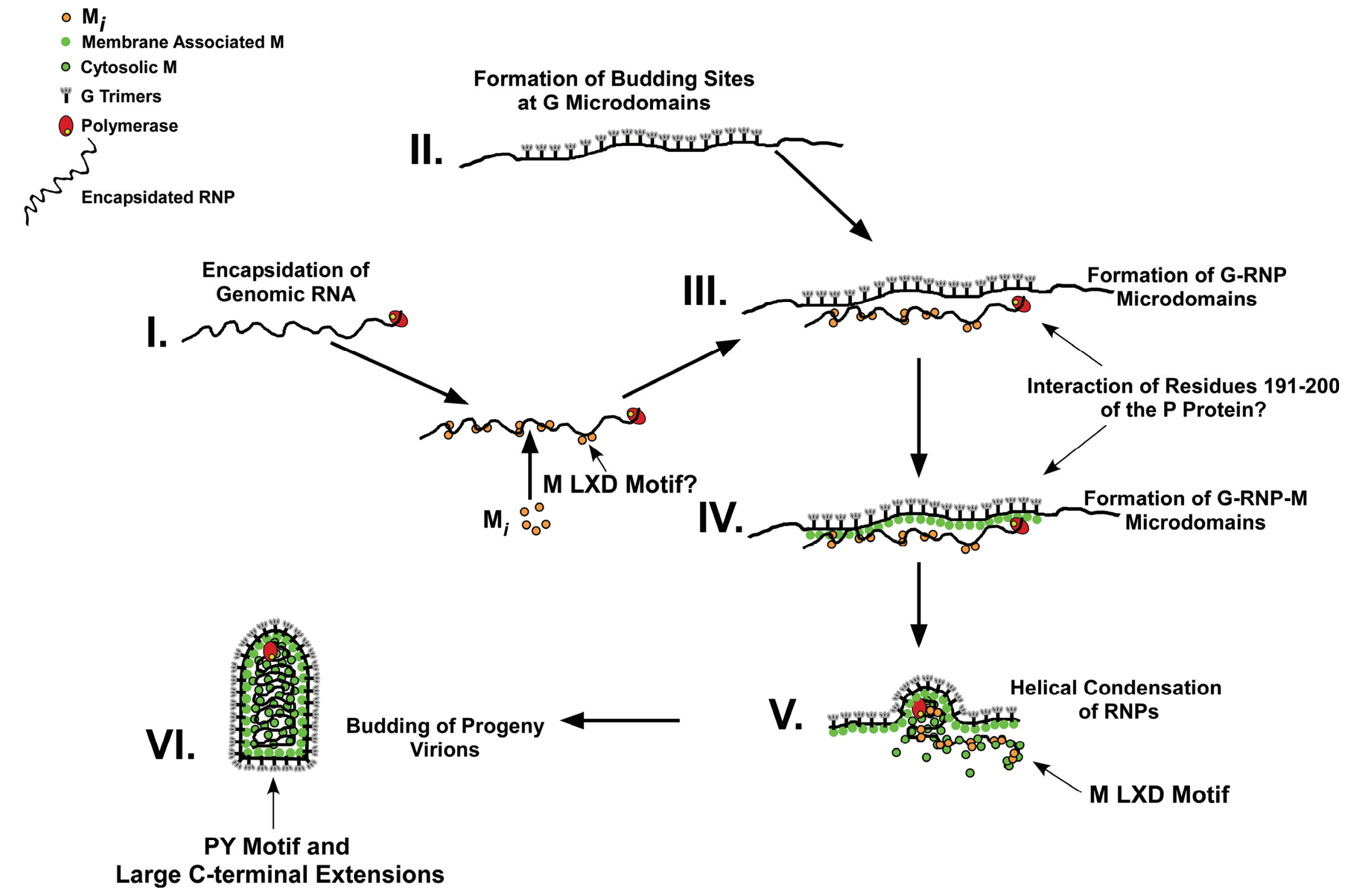


\section{VSV Translation}

Recently, a new model has been proposed for translation of viral proteins during infection where dephosphorylation of eIF4E results in inhibition of host protein synthesis and the host mRNA is then shuttled to inactive mRNPs which in turn allows for newly made viral mRNA to be translated (208). The translation of newly made viral mRNA which occurs between 4 to 8 hpi, may be enhanced by M $(13,35,208)$. In Chapter 4 we observed a reduction in viral protein synthesis between 4 and 8 hpi and I propose here that the M D125A loop mutation results in the inability of the mutant to support viral protein synthesis.

Based on the new virus translation regulation model mentioned above, our model proposes that $\mathrm{M}$ protein supports viral translation by playing a supporting role, after the dephosphorylation eIF4E, for cap-dependent translation of newly synthesized viral transcripts. Based on the D125A phenotype, the exposed loop of M (amino acids 121 to 125) is involved in this regulation (Fig. 5-3). It has to be said that the protein translation and assembly effects have not been separated and for now we designate this loop as important for both of these events. The alignment of proteins retreived from a PubMed blast search of the exposed loop of M revealed a potential LXD motif that could be important in one or both of the phenotypes observed for the loop mutants.

\section{Future Directions}

The models presented here are a combination of the current views from the literature and my work. However, these models require further investigation to clarify the "black boxes" that still exist. In the entry model (Fig. 5-1) for instance, the exact location of M that associates with the recycling endosomes after VSV entry can be visualized using photoconversion of diaminobenzidine (DAB) by Lumio-Red ${ }^{\mathrm{TM}}$ from rVSV-M-Lumio-Red virus for high resolution TEM. In the assembly model (Fig. 5-2), the condensed nature of the LXD mutant RNPs can be further examined by light scattering analysis when compared to wild-type. The spacing between the helical turns of the RNPs in TEM visualization could also be measured and analyzed for statistical significance to determine if there is any difference between the LXD mutant and wildtype RNPs. In the translation model (Fig. 5-3), the exact mechanism for how M supports viral protein synthesis from viral mRNA still needs to be examined. In the model dephosphorylated eIF4E is present in the initiation complex and $\mathrm{M}$ is depicted in support but it may be that $\mathrm{M}$ replaces eIF4E in this complex altogether. Further investigation into the idea that M may replace eIF4E is intriguing since the proteins are similar in size, have a pleiomorphic $\mathrm{N}$-terminus, and similar crystal structure $(20,66)$. This is particularly interesting since there is an unanswered question about why $\mathrm{M}$ is phosphorylated $(98,99)$ where phosphorylation may be required for the support of viral protein synthesis after 4 hpi. Analysis of viral protein synthesis after 4 hpi using phosphorylation site mutants could certainly a way to examine this hypothesis. While there are "black boxes" that still 


\section{Cellular Protein \\ Synthesis}
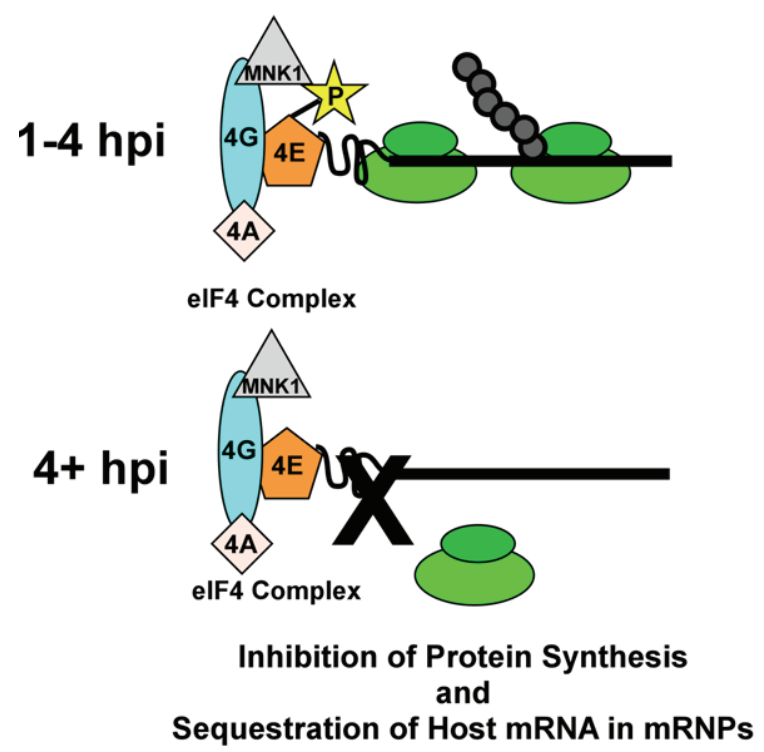

Viral Protein

Synthesis
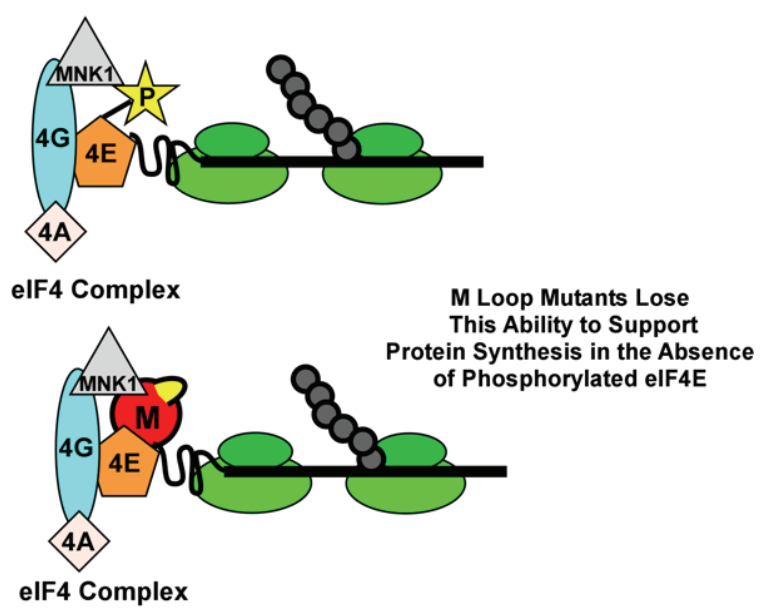

Newly Made Viral mRNA Translated

Through M Rescue of elF4E Activity

\section{Enhancement of the elF4F Complex}

Figure 5-3. VSV translational support model for $M$.

(Left-hand column) After 4 hours post infection (hpi) cellular protein synthesis is inhibited by dephosphorylation of eIF4E and the sequestration of host mRNA to mRNPs $(33,208)$. However, this model proposes that $M$ can support viral protein synthesis after the dephosphorylation of eIF4E and the loop (yellow loop on red $\mathrm{M}$ ) is important for this especially the LXD sequence of $\mathrm{M}$, however the manner in which this occurs is open for speculation. 
exist in the models for VSV replication, additions to these models that help clarify the "black boxes" are important to the contribution of VSV as the prototype Rhabdovirus and may contribute to insights into replication of viruses within the order Mononegavirales. 


\section{List of References}

1. [Anon]. 2006. Vesicular stomatitis affects multiple states in 2005. Javma-Journal of the American Veterinary Medical Association 228:19.

2. Adams, S. R., R. E. Campbell, L. A. Gross, B. R. Martin, G. K. Walkup, Y. Yao, J. Llopis, and R. Y. Tsien. 2002. New biarsenical ligands and tetracysteine motifs for protein labeling in vitro and in vivo: synthesis and biological applications. J Am Chem Soc 124:6063-76.

3. Ahmed, M., and D. S. Lyles. 1998. Effect of vesicular stomatitis virus matrix protein on transcription directed by host RNA polymerases I, II, and III. J Virol 72:8413-9.

4. Ahmed, M., M. O. McKenzie, S. Puckett, M. Hojnacki, L. Poliquin, and D. S. Lyles. 2003. Ability of the matrix protein of vesicular stomatitis virus to suppress beta interferon gene expression is genetically correlated with the inhibition of host RNA and protein synthesis. J Virol 77:4646-57.

5. Arhel, N., A. Genovesio, K. A. Kim, S. Miko, E. Perret, J. C. Olivo-Marin, S. Shorte, and P. Charneau. 2006. Quantitative four-dimensional tracking of cytoplasmic and nuclear HIV-1 complexes. Nat Methods 3:817-24.

6. Arora, K. K., and J. F. Williams. 1987. Introduction and metabolism of pentose and hexose phosphates in permeabilized Morris hepatoma 5123TC cells. Cell Biochem Funct 5:289-300.

7. Baltimore, D. 1980. Evolution of RNA viruses. Ann N Y Acad Sci 354:492-7.

8. Barber, G. N. 2004. Vesicular stomatitis virus as an oncolytic vector. Viral Immunol 17:516-27.

9. Barber, G. N. 2005. VSV-tumor selective replication and protein translation. Oncogene 24:7710-9.

10. Barge, A., J. Gagnon, A. Chaffotte, P. Timmins, J. Langowski, R. W. Ruigrok, and Y. Gaudin. 1996. Rod-like shape of vesicular stomatitis virus matrix protein. Virology 219:465-70.

11. Barge, A., Y. Gaudin, P. Coulon, and R. W. Ruigrok. 1993. Vesicular stomatitis virus M protein may be inside the ribonucleocapsid coil. J Virol 67:7246-53.

12. Barzilay, E., N. Ben-Califa, K. Hirschberg, and D. Neumann. 2005. Uncoupling of brefeldin a-mediated coatomer protein complex-I dissociation from Golgi redistribution. Traffic 6:794-802.

13. Black, B. L., G. Brewer, and D. S. Lyles. 1994. Effect of vesicular stomatitis virus matrix protein on host-directed translation in vivo. J Virol 68:555-60.

14. Black, B. L., and D. S. Lyles. 1992. Vesicular stomatitis virus matrix protein inhibits host cell-directed transcription of target genes in vivo. J Virol 66: 4058-64.

15. Black, B. L., R. B. Rhodes, M. McKenzie, and D. S. Lyles. 1993. The role of vesicular stomatitis virus matrix protein in inhibition of host-directed gene expression is genetically separable from its function in virus assembly. J Virol 67:4814-21. 
16. Blondel, D., G. G. Harmison, and M. Schubert. 1990. Role of matrix protein in cytopathogenesis of vesicular stomatitis virus. J Virol 64:1716-25.

17. Blower, M. D., M. Nachury, R. Heald, and K. Weis. 2005. A Rae1-containing ribonucleoprotein complex is required for mitotic spindle assembly. Cell 121: 223-34.

18. Blumenthal, R., A. Bali-Puri, A. Walter, D. Covell, and O. Eidelman. 1987. $\mathrm{pH}$-dependent fusion of vesicular stomatitis virus with Vero cells. Measurement by dequenching of octadecyl rhodamine fluorescence. J Biol Chem 262:13614-9.

19. Bridges, V. E., B. J. McCluskey, M. D. Salman, H. S. Hurd, and J. Dick. 1997. Review of the 1995 vesicular stomatitis outbreak in the western United States. J Am Vet Med Assoc 211:556-60.

20. Brown, C. J., I. McNae, P. M. Fischer, and M. D. Walkinshaw. 2007. Crystallographic and mass spectrometric characterisation of eIF4E with N7alkylated cap derivatives. J Mol Biol 372:7-15.

21. Brown, E. L., and D. S. Lyles. 2003. A novel method for analysis of membrane microdomains: vesicular stomatitis virus glycoprotein microdomains change in size during infection, and those outside of budding sites resemble sites of virus budding. Virology 310:343-58.

22. Brown, J. C., W. W. Newcomb, and S. Lawrenz-Smith. 1988. pH-dependent accumulation of the vesicular stomatitis virus glycoprotein at the ends of intact virions. Virology 167:625-9.

23. Brumell, J. H., and M. A. Scidmore. 2007. Manipulation of rab GTPase function by intracellular bacterial pathogens. Microbiol Mol Biol Rev 71:636-52.

24. Bui, M., G. Whittaker, and A. Helenius. 1996. Effect of M1 protein and low pH on nuclear transport of influenza virus ribonucleoproteins. J Virol 70:8391-401.

25. Campbell, E. M., O. Perez, M. Melar, and T. J. Hope. 2007. Labeling HIV-1 virions with two fluorescent proteins allows identification of virions that have productively entered the target cell. Virology 360:286-93.

26. Carroll, A. R., and R. R. Wagner. 1979. Role of the membrane (M) protein in endogenous inhibition of in vitro transcription by vesicular stomatitis virus. $\mathrm{J}$ Virol 29:134-42.

27. Casanova, J. E. 2007. Regulation of Arf activation: the Sec 7 family of guanine nucleotide exchange factors. Traffic 8:1476-85.

28. Charpilienne, A., M. Nejmeddine, M. Berois, N. Parez, E. Neumann, E. Hewat, G. Trugnan, and J. Cohen. 2001. Individual rotavirus-like particles containing 120 molecules of fluorescent protein are visible in living cells. J Biol Chem 276:29361-7.

29. Chen, S. S., N. Ariel, and A. S. Huang. 1988. Membrane anchors of vesicular stomatitis virus: characterization and incorporation into virions. J Virol 62: 2552-6.

30. Chong, L. D., and J. K. Rose. 1994. Interactions of normal and mutant vesicular stomatitis virus matrix proteins with the plasma membrane and nucleocapsids. J Virol 68:441-7.

31. Chong, L. D., and J. K. Rose. 1993. Membrane association of functional vesicular stomatitis virus matrix protein in vivo. J Virol 67:407-14. 
32. Clinton, G. M., S. P. Little, F. S. Hagen, and A. S. Huang. 1978. The matrix (M) protein of vesicular stomatitis virus regulates transcription. Cell 15:1455-62.

33. Connor, J. H., and D. S. Lyles. 2005. Inhibition of host and viral translation during vesicular stomatitis virus infection. eIF2 is responsible for the inhibition of viral but not host translation. J Biol Chem 280:13512-9.

34. Connor, J. H., and D. S. Lyles. 2002. Vesicular stomatitis virus infection alters the eIF4F translation initiation complex and causes dephosphorylation of the eIF4E binding protein 4E-BP1. J Virol 76:10177-87.

35. Connor, J. H., M. O. McKenzie, and D. S. Lyles. 2006. Role of residues 121 to 124 of vesicular stomatitis virus matrix protein in virus assembly and virus-host interaction. J Virol 80:3701-11.

36. Connor, J. H., C. Naczki, C. Koumenis, and D. S. Lyles. 2004. Replication and cytopathic effect of oncolytic vesicular stomatitis virus in hypoxic tumor cells in vitro and in vivo. J Virol 78:8960-70.

37. Copeland, K. F., and J. L. Heeney. 1996. T helper cell activation and human retroviral pathogenesis. Microbiol Rev 60:722-42.

38. Cotton, W. 1926. The causal agent of vesicular stomatitis proved to be a filterpassing virus. J Am Vet Med Assoc 23:168-179.

39. Cotton, W. 1927. Vesicular stomatitis. J Am Vet Med Assoc 22:169-175.

40. Craven, R. C., R. N. Harty, J. Paragas, P. Palese, and J. W. Wills. 1999. Late domain function identified in the vesicular stomatitis virus $\mathrm{M}$ protein by use of rhabdovirus-retrovirus chimeras. J Virol 73:3359-65.

41. Dalton, K. P., and J. K. Rose. 2001. Vesicular stomatitis virus glycoprotein containing the entire green fluorescent protein on its cytoplasmic domain is incorporated efficiently into virus particles. Virology 279:414-21.

42. Das, S. C., D. Nayak, Y. Zhou, and A. K. Pattnaik. 2006. Visualization of intracellular transport of vesicular stomatitis virus nucleocapsids in living cells. $\mathrm{J}$ Virol 80:6368-77.

43. Das, S. C., and A. K. Pattnaik. 2005. Role of the hypervariable hinge region of phosphoprotein $\mathrm{P}$ of vesicular stomatitis virus in viral RNA synthesis and assembly of infectious virus particles. J Virol 79:8101-12.

44. De, B. P., G. B. Thornton, D. Luk, and A. K. Banerjee. 1982. Purified matrix protein of vesicular stomatitis virus blocks viral transcription in vitro. Proc Natl Acad Sci U S A 79:7137-41.

45. Dohner, K., C. H. Nagel, and B. Sodeik. 2005. Viral stop-and-go along microtubules: taking a ride with dynein and kinesins. Trends Microbiol 13:320-7.

46. Dubovi, E. J., and R. R. Wagner. 1977. Spatial relationships of the proteins of vesicular stomatitis virus: induction of reversible oligomers by cleavable protein cross-linkers and oxidation. J Virol 22:500-9.

47. Dunigan, D. D., S. Baird, and J. Lucas-Lenard. 1986. Lack of correlation between the accumulation of plus-strand leader RNA and the inhibition of protein and RNA synthesis in vesicular stomatitis virus infected mouse L cells. Virology 150:231-46.

48. Dunigan, D. D., and J. M. Lucas-Lenard. 1983. Two transcription products of the vesicular stomatitis virus genome may control L-cell protein synthesis. J Virol 45:618-26. 
49. Duntsch, C. D., Q. Zhou, H. R. Jayakar, J. D. Weimar, J. H. Robertson, L. M. Pfeffer, L. Wang, Z. Xiang, and M. A. Whitt. 2004. Recombinant vesicular stomatitis virus vectors as oncolytic agents in the treatment of high-grade gliomas in an organotypic brain tissue slice-glioma coculture model. J Neurosurg 100:1049-59.

50. Ebert, O., S. Harbaran, K. Shinozaki, and S. L. Woo. 2005. Systemic therapy of experimental breast cancer metastases by mutant vesicular stomatitis virus in immune-competent mice. Cancer Gene Ther 12:350-8.

51. Eidelman, O., R. Schlegel, T. S. Tralka, and R. Blumenthal. 1984. pHdependent fusion induced by vesicular stomatitis virus glycoprotein reconstituted into phospholipid vesicles. J Biol Chem 259:4622-8.

52. Fan, D. P., and B. M. Sefton. 1978. The entry into host cells of Sindbis virus, vesicular stomatitis virus and Sendai virus. Cell 15:985-92.

53. Faria, P. A., P. Chakraborty, A. Levay, G. N. Barber, H. J. Ezelle, J. Enninga, C. Arana, J. van Deursen, and B. M. Fontoura. 2005. VSV disrupts the Rae1/mrnp41 mRNA nuclear export pathway. Mol Cell 17:93-102.

54. Feng, Y., B. Press, and A. Wandinger-Ness. 1995. Rab 7: an important regulator of late endocytic membrane traffic. J Cell Biol 131:1435-52.

55. Ferran, M. C., and J. M. Lucas-Lenard. 1997. The vesicular stomatitis virus matrix protein inhibits transcription from the human beta interferon promoter. $\mathrm{J}$ Virol 71:371-7.

56. Fields, B. N., and K. Hawkins. 1967. Human infection with the virus of vesicular stomatitis during an epizootic. N Engl J Med 277:989-94.

57. Flood, E. A., and D. S. Lyles. 1999. Assembly of nucleocapsids with cytosolic and membrane-derived matrix proteins of vesicular stomatitis virus. Virology 261:295-308.

58. Florkiewicz, R. Z., and J. K. Rose. 1984. A cell line expressing vesicular stomatitis virus glycoprotein fuses at low pH. Science 225:721-3.

59. Fredericksen, B. L., and M. A. Whitt. 1998. Attenuation of recombinant vesicular stomatitis viruses encoding mutant glycoproteins demonstrate a critical role for maintaining a high $\mathrm{pH}$ threshold for membrane fusion in viral fitness. Virology 240:349-58.

60. Fresco, L. D., M. G. Kurilla, and J. D. Keene. 1987. Rapid inhibition of processing and assembly of small nuclear ribonucleoproteins after infection with vesicular stomatitis virus. Mol Cell Biol 7:1148-55.

61. Fuerst, T. R., P. L. Earl, and B. Moss. 1987. Use of a hybrid vaccinia virus-T7 RNA polymerase system for expression of target genes. Mol Cell Biol 7:2538-44.

62. Gaddy, D. F., and D. S. Lyles. 2007. Oncolytic vesicular stomatitis virus induces apoptosis via signaling through PKR, Fas, and Daxx. J Virol 81:2792-804.

63. Gaddy, D. F., and D. S. Lyles. 2005. Vesicular stomatitis viruses expressing wild-type or mutant $\mathrm{M}$ proteins activate apoptosis through distinct pathways. J Virol 79:4170-9.

64. Gaietta, G., T. J. Deerinck, S. R. Adams, J. Bouwer, O. Tour, D. W. Laird, G. E. Sosinsky, R. Y. Tsien, and M. H. Ellisman. 2002. Multicolor and electron microscopic imaging of connexin trafficking. Science 296:503-7. 
65. Gaudier, M., Y. Gaudin, and M. Knossow. 2001. Cleavage of vesicular stomatitis virus matrix protein prevents self-association and leads to crystallization. Virology 288:308-14.

66. Gaudier, M., Y. Gaudin, and M. Knossow. 2002. Crystal structure of vesicular stomatitis virus matrix protein. Embo J 21:2886-92.

67. Gaudin, Y., A. Barge, C. Ebel, and R. W. Ruigrok. 1995. Aggregation of VSV $\mathrm{M}$ protein is reversible and mediated by nucleation sites: implications for viral assembly. Virology 206:28-37.

68. Gaudin, Y., J. Sturgis, M. Doumith, A. Barge, B. Robert, and R. W. Ruigrok. 1997. Conformational flexibility and polymerization of vesicular stomatitis virus matrix protein. J Mol Biol 274:816-25.

69. Glodowski, D. R., J. M. Petersen, and J. E. Dahlberg. 2002. Complex nuclear localization signals in the matrix protein of vesicular stomatitis virus. J Biol Chem 277:46864-70.

70. Griffin, B. A., S. R. Adams, and R. Y. Tsien. 1998. Specific covalent labeling of recombinant protein molecules inside live cells. Science 281:269-72.

71. Grinnell, B. W., and R. R. Wagner. 1983. Comparative inhibition of cellular transcription by vesicular stomatitis virus serotypes New Jersey and Indiana: role of each viral leader RNA. J Virol 48:88-101.

72. Grinnell, B. W., and R. R. Wagner. 1985. Inhibition of DNA-dependent transcription by the leader RNA of vesicular stomatitis virus: role of specific nucleotide sequences and cell protein binding. Mol Cell Biol 5:2502-13.

73. Grinnell, B. W., and R. R. Wagner. 1984. Nucleotide sequence and secondary structure of VSV leader RNA and homologous DNA involved in inhibition of DNA-dependent transcription. Cell 36:533-43.

74. Gruenberg, J., G. Griffiths, and K. E. Howell. 1989. Characterization of the early endosome and putative endocytic carrier vesicles in vivo and with an assay of vesicle fusion in vitro. J Cell Biol 108:1301-16.

75. Gruenberg, J., and F. R. Maxfield. 1995. Membrane transport in the endocytic pathway. Curr Opin Cell Biol 7:552-63.

76. Han, Z., J. M. Licata, J. Paragas, and R. N. Harty. 2007. Permeabilization of the plasma membrane by Ebola virus GP2. Virus Genes 34:273-81.

77. Harris, A., B. Sha, and M. Luo. 1999. Structural similarities between influenza virus matrix protein M1 and human immunodeficiency virus matrix and capsid proteins: an evolutionary link between negative-stranded RNA viruses and retroviruses. J Gen Virol 80 ( Pt 4):863-9.

78. Harty, R. N., M. E. Brown, J. P. McGettigan, G. Wang, H. R. Jayakar, J. M. Huibregtse, M. A. Whitt, and M. J. Schnell. 2001. Rhabdoviruses and the cellular ubiquitin-proteasome system: a budding interaction. J Virol 75:10623-9.

79. Harty, R. N., M. E. Brown, G. L. Wang, J. Huibregtse, and F. P. Hayes. 2000. A PPxY motif within the VP40 protein of Ebola virus interacts physically and functionally with a ubiquitin ligase: Implications for filovirus budding. Proceedings of the National Academy of Sciences of the United States of America 97:13871-13876.

80. Harty, R. N., J. Paragas, M. Sudol, and P. Palese. 1999. A proline-rich motif within the matrix protein of vesicular stomatitis virus and rabies virus interacts 
with WW domains of cellular proteins: implications for viral budding. J Virol 73:2921-9.

81. Helenius, A. 1992. Unpacking the incoming influenza virus. Cell 69:577-8.

82. Helenius, A., M. Marsh, and J. White. 1982. Inhibition of Semliki forest virus penetration by lysosomotropic weak bases. J Gen Virol 58 Pt 1:47-61.

83. Hellen, C. U., and E. Wimmer. 1995. Translation of encephalomyocarditis virus RNA by internal ribosomal entry. Curr Top Microbiol Immunol 203:31-63.

84. Her, L. S., E. Lund, and J. E. Dahlberg. 1997. Inhibition of Ran guanosine triphosphatase-dependent nuclear transport by the matrix protein of vesicular stomatitis virus. Science 276:1845-8.

85. Hercyk, N., S. M. Horikami, and S. A. Moyer. 1988. The vesicular stomatitis virus L protein possesses the mRNA methyltransferase activities. Virology 163:222-5.

86. Irie, T., E. Carnero, A. Okumura, A. Garcia-Sastre, and R. N. Harty. 2007. Modifications of the PSAP region of the matrix protein lead to attenuation of vesicular stomatitis virus in vitro and in vivo. J Gen Virol 88:2559-67.

87. Iverson, L. E., and J. K. Rose. 1981. Localized attenuation and discontinuous synthesis during vesicular stomatitis virus transcription. Cell 23:477-84.

88. Iverson, L. E., and J. K. Rose. 1982. Sequential synthesis of 5'-proximal vesicular stomatitis virus mRNA sequences. J Virol 44:356-65.

89. Jang, S. K., T. V. Pestova, C. U. Hellen, G. W. Witherell, and E. Wimmer. 1990. Cap-independent translation of picornavirus RNAs: structure and function of the internal ribosomal entry site. Enzyme 44:292-309.

90. Jayakar, H. R., E. Jeetendra, and M. A. Whitt. 2004. Rhabdovirus assembly and budding. Virus Research 106:117-132.

91. Jayakar, H. R., K. G. Murti, and M. A. Whitt. 2000. Mutations in the PPPY motif of vesicular stomatitis virus matrix protein reduce virus budding by inhibiting a late step in virion release. J Virol 74:9818-27.

92. Jayakar, H. R., and M. A. Whitt. 2002. Identification of two additional translation products from the matrix $(\mathrm{M})$ gene that contribute to vesicular stomatitis virus cytopathology. J Virol 76:8011-8.

93. Jeetendra, E., K. Ghosh, D. Odell, J. Li, H. P. Ghosh, and M. A. Whitt. 2003. The membrane-proximal region of vesicular stomatitis virus glycoprotein $\mathrm{G}$ ectodomain is critical for fusion and virus infectivity. J Virol 77:12807-18.

94. Jeetendra, E., C. S. Robison, L. M. Albritton, and M. A. Whitt. 2002. The membrane-proximal domain of vesicular stomatitis virus $\mathrm{G}$ protein functions as a membrane fusion potentiator and can induce hemifusion. J Virol 76:12300-11.

95. Johnson, J. E., M. J. Schnell, L. Buonocore, and J. K. Rose. 1997. Specific targeting to CD4+ cells of recombinant vesicular stomatitis viruses encoding human immunodeficiency virus envelope proteins. J Virol 71:5060-8.

96. Johnson, K. M., R. B. Tesh, and P. H. Peralta. 1969. Epidemiology of vesicular stomatitis virus: some new data and a hypothesis for transmission of the Indian serotype. J Am Vet Med Assoc 155:2133-40.

97. Justice, P. A., W. Sun, Y. Li, Z. Ye, P. R. Grigera, and R. R. Wagner. 1995. Membrane vesiculation function and exocytosis of wild-type and mutant matrix proteins of vesicular stomatitis virus. J Virol 69:3156-60. 
98. Kaptur, P. E., B. J. McCreedy, Jr., and D. S. Lyles. 1992. Sites of in vivo phosphorylation of vesicular stomatitis virus matrix protein. J Virol 66:5384-92.

99. Kaptur, P. E., M. O. McKenzie, G. W. Wertz, and D. S. Lyles. 1995. Assembly functions of vesicular stomatitis virus matrix protein are not disrupted by mutations at major sites of phosphorylation. Virology 206:894-903.

100. Kaptur, P. E., R. B. Rhodes, and D. S. Lyles. 1991. Sequences of the vesicular stomatitis virus matrix protein involved in binding to nucleocapsids. J Virol 65:1057-65.

101. Kasermann, F., and C. Kempf. 1996. Low pH-induced pore formation by spike proteins of enveloped viruses. J Gen Virol 77 ( Pt 12):3025-32.

102. Kemler, I., G. Whittaker, and A. Helenius. 1994. Nuclear import of microinjected influenza virus ribonucleoproteins. Virology 202:1028-33.

103. Kikonyogo, A., F. Bouamr, M. L. Vana, Y. Xiang, A. Aiyar, C. Carter, and J. Leis. 2001. Proteins related to the Nedd4 family of ubiquitin protein ligases interact with the L domain of Rous sarcoma virus and are required for gag budding from cells. Proceedings of the National Academy of Sciences of the United States of America 98:11199-11204.

104. Kim, G. N., and C. Y. Kang. 2007. Matrix protein of VSV New Jersey serotype containing methionine to arginine substitutions at positions 48 and 51 allows near-normal host cell gene expression. Virology 357:41-53.

105. Kopecky, S. A., and D. S. Lyles. 2003. The cell-rounding activity of the vesicular stomatitis virus matrix protein is due to the induction of cell death. $\mathrm{J}$ Virol 77:5524-8.

106. Kopecky, S. A., M. C. Willingham, and D. S. Lyles. 2001. Matrix protein and another viral component contribute to induction of apoptosis in cells infected with vesicular stomatitis virus. J Virol 75:12169-81.

107. Koyama, A. H. 1995. Induction of Apoptotic DNA Fragmentation by the Infection of Vesicular Stomatitis-Virus. Virus Research 37:285-290.

108. Kurilla, M. G., H. Piwnica-Worms, and J. D. Keene. 1982. Rapid and transient localization of the leader RNA of vesicular stomatitis virus in the nuclei of infected cells. Proc Natl Acad Sci U S A 79:5240-4.

109. Lam, V., K. A. Duca, and J. Yin. 2005. Arrested spread of vesicular stomatitis virus infections in vitro depends on interferon-mediated antiviral activity. Biotechnol Bioeng 90:793-804.

110. Lamb, R. A., S. L. Zebedee, and C. D. Richardson. 1985. Influenza virus M2 protein is an integral membrane protein expressed on the infected-cell surface. Cell 40:627-33.

111. Landry, M. L., D. R. Mayo, and G. D. Hsiung. 1982. Comparison of guinea pig embryo cells, rabbit kidney cells, and human embryonic lung fibroblast cell strains for isolation of herpes simplex virus. J Clin Microbiol 15:842-7.

112. Lanzrein, M., N. Kasermann, and C. Kempf. 1992. Changes in membrane permeability during Semliki Forest virus induced cell fusion. Biosci Rep 12: 221-36.

113. Lanzrein, M., N. Kasermann, R. Weingart, and C. Kempf. 1993. Early events of Semliki Forest virus-induced cell-cell fusion. Virology 196:541-7. 
114. Lanzrein, M., A. Schlegel, and C. Kempf. 1994. Entry and uncoating of enveloped viruses. Biochem J 302 ( Pt 2):313-20.

115. Lanzrein, M., R. Weingart, and C. Kempf. 1993. pH-dependent pore formation in Semliki forest virus-infected Aedes albopictus cells. Virology 193:296-302.

116. Lawson, N. D., E. A. Stillman, M. A. Whitt, and J. K. Rose. 1995. Recombinant vesicular stomatitis viruses from DNA. Proc Natl Acad Sci U S A 92:4477-81.

117. Le Blanc, I., P. P. Luyet, V. Pons, C. Ferguson, N. Emans, A. Petiot, N. Mayran, N. Demaurex, J. Faure, R. Sadoul, R. G. Parton, and J. Gruenberg. 2005. Endosome-to-cytosol transport of viral nucleocapsids. Nat Cell Biol 7: 653-64.

118. Lee, H. K., J. M. Lund, B. Ramanathan, N. Mizushima, and A. Iwasaki. 2007. Autophagy-dependent viral recognition by plasmacytoid dendritic cells. Science 315:1398-401.

119. Lenard, J. 1996. Negative-strand virus M and retrovirus MA proteins: all in a family? Virology 216:289-98.

120. Lenard, J., and R. Vanderoef. 1990. Localization of the membrane-associated region of vesicular stomatitis virus $\mathrm{M}$ protein at the $\mathrm{N}$ terminus, using the hydrophobic, photoreactive probe 125I-TID. J Virol 64:3486-91.

121. Letchworth, G. J., L. L. Rodriguez, and J. Del cbarrera. 1999. Vesicular stomatitis. Vet J 157:239-60.

122. Li, Y., L. Z. Luo, R. M. Snyder, and R. R. Wagner. 1988. Expression of the M gene of vesicular stomatitis virus cloned in various vaccinia virus vectors. J Virol 62:776-82.

123. Lichty, B. D., H. McBride, S. Hanson, and J. C. Bell. 2006. Matrix protein of Vesicular stomatitis virus harbours a cryptic mitochondrial-targeting motif. J Gen Virol 87:3379-84.

124. Lorenz, J. D., J. F. Watkins, and M. J. Smerdon. 1988. Excision repair of UV damage in human fibroblasts reversibly permeabilized by lysolecithin. Mutat Res 193:167-79.

125. Luan, P., and M. Glaser. 1994. Formation of membrane domains by the envelope proteins of vesicular stomatitis virus. Biochemistry 33:4483-9.

126. Luan, P., L. Yang, and M. Glaser. 1995. Formation of membrane domains created during the budding of vesicular stomatitis virus. A model for selective lipid and protein sorting in biological membranes. Biochemistry 34:9874-83.

127. Lun, X., D. L. Senger, T. Alain, A. Oprea, K. Parato, D. Stojdl, B. Lichty, A. Power, R. N. Johnston, M. Hamilton, I. Parney, J. C. Bell, and P. A. Forsyth. 2006. Effects of intravenously administered recombinant vesicular stomatitis virus (VSV(deltaM51)) on multifocal and invasive gliomas. J Natl Cancer Inst 98: 1546-57.

128. Lyles, D. S., M. McKenzie, and J. W. Parce. 1992. Subunit interactions of vesicular stomatitis virus envelope glycoprotein stabilized by binding to viral matrix protein. J Virol 66:349-58.

129. Lyles, D. S., and M. O. McKenzie. 1997. Activity of vesicular stomatitis virus M protein mutants in cell rounding is correlated with the ability to inhibit host gene expression and is not correlated with virus assembly function. Virology 229: 
77-89.

130. Lyles, D. S., and M. O. McKenzie. 1998. Reversible and irreversible steps in assembly and disassembly of vesicular stomatitis virus: equilibria and kinetics of dissociation of nucleocapsid-M protein complexes assembled in vivo.

Biochemistry 37:439-50.

131. Lyles, D. S., M. O. McKenzie, M. Ahmed, and S. C. Woolwine. 1996. Potency of wild-type and temperature-sensitive vesicular stomatitis virus matrix protein in the inhibition of host-directed gene expression. Virology 225:172-80.

132. Lyles, D. S., M. O. McKenzie, P. E. Kaptur, K. W. Grant, and W. G. Jerome. 1996. Complementation of $M$ gene mutants of vesicular stomatitis virus by plasmid-derived $\mathrm{M}$ protein converts spherical extracellular particles into native bullet shapes. Virology 217:76-87.

133. Lyles, D. S., L. Puddington, and B. J. McCreedy, Jr. 1988. Vesicular stomatitis virus $\mathrm{M}$ protein in the nuclei of infected cells. J Virol 62:4387-92.

134. Mannen, K., M. Ohuchi, and K. Mifune. 1982. pH-dependent hemolysis and cell fusion of rhabdoviruses. Microbiol Immunol 26:1035-43.

135. Marsh, M., and R. Bron. 1997. SFV infection in CHO cells: cell-type specific restrictions to productive virus entry at the cell surface. J Cell Sci 110 ( Pt 1): 95-103.

136. Marsh, M., and A. Helenius. 2006. Virus entry: open sesame. Cell 124:729-40.

137. Martin, K., and A. Helenius. 1991. Nuclear transport of influenza virus ribonucleoproteins: the viral matrix protein (M1) promotes export and inhibits import. Cell 67:117-30.

138. Marvaldi, J. L., J. Lucas-Lenard, M. J. Sekellick, and P. I. Marcus. 1977. Cell killing by viruses. IV. Cell killing and protein synthesis inhibition by vesicular stomatitis virus require the same gene functions. Virology 79:267-80.

139. Matlin, K. S., H. Reggio, A. Helenius, and K. Simons. 1982. Pathway of vesicular stomatitis virus entry leading to infection. J Mol Biol 156:609-31.

140. Matthews, R. E. 1985. Viral taxonomy for the nonvirologist. Annu Rev Microbiol 39:451-74.

141. McClure, M. O., M. Marsh, and R. A. Weiss. 1988. Human immunodeficiency virus infection of CD4-bearing cells occurs by a $\mathrm{pH}$-independent mechanism. Embo J 7:513-8.

142. McClure, M. O., M. A. Sommerfelt, M. Marsh, and R. A. Weiss. 1990. The $\mathrm{pH}$ independence of mammalian retrovirus infection. J Gen Virol 71 ( $\mathbf{P t ~ 4 ) : ~}$ 767-73.

143. McCluskey, B. J., B. J. Beaty, and M. D. Salman. 2003. Climatic factors and the occurence of vesicular stomatitis in New Mexico, United States of America. Revue Scientifique Et Technique De L Office International Des Epizooties 22:849-856.

144. McCluskey, B. J., H. S. Hurd, and E. L. Mumford. 1999. Review of the 1997 outbreak of vesicular stomatitis in the western United States. J Am Vet Med Assoc 215:1259-62.

145. McCombs, R. M., M. B. Melnick, and J. P. Brunschwig. 1966. Biophysical studies of vesicular stomatitis virus. J Bacteriol 91:803-12. 
146. McCreedy, B. J., Jr., and D. S. Lyles. 1989. Distribution of M protein and nucleocapsid protein of vesicular stomatitis virus in infected cell plasma membranes. Virus Res 14:189-205.

147. McCreedy, B. J., Jr., K. P. McKinnon, and D. S. Lyles. 1990. Solubility of vesicular stomatitis virus $\mathrm{M}$ protein in the cytosol of infected cells or isolated from virions. J Virol 64:902-6.

148. McGowan, J. J., S. U. Emerson, and R. R. Wagner. 1982. The plus-strand leader RNA of VSV inhibits DNA-dependent transcription of adenovirus and SV40 genes in a soluble whole-cell extract. Cell 28:325-33.

149. Mebatsion, T., M. Konig, and K. K. Conzelmann. 1996. Budding of rabies virus particles in the absence of the spike glycoprotein. Cell 84:941-51.

150. Melki, R., Y. Gaudin, and D. Blondel. 1994. Interaction between tubulin and the viral matrix protein of vesicular stomatitis virus: possible implications in the viral cytopathic effect. Virology 202:339-47.

151. Morrison, T. G., and C. O. McQuain. 1978. Assembly of viral membranes: nature of the association of vesicular stomatitis virus proteins to membranes. $\mathrm{J}$ Virol 26:115-25.

152. Muller, B., J. Daecke, O. T. Fackler, M. T. Dittmar, H. Zentgraf, and H. G. Krausslich. 2004. Construction and characterization of a fluorescently labeled infectious human immunodeficiency virus type 1 derivative. J Virol 78:10803-13.

153. Newcomb, W. W., and J. C. Brown. 1981. Role of the vesicular stomatitis virus matrix protein in maintaining the viral nucleocapsid in the condensed form found in native virions. J Virol 39:295-9.

154. Newcomb, W. W., G. J. Tobin, J. J. McGowan, and J. C. Brown. 1982. In vitro reassembly of vesicular stomatitis virus skeletons. J Virol 41:1055-62.

155. Nishiyama, Y., Y. Ito, K. Shimokata, I. Nagata, N. Kurachi, and Y. Sugiura. 1977. Comparative studies on cytopathic effects induced by vesicular stomatitis virus in two cell types. Microbiol Immunol 21:693-702.

156. Noser, J. A., A. A. Mael, R. Sakuma, S. Ohmine, P. Marcato, P. W. Lee, and Y. Ikeda. 2007. The RAS/Raf1/MEK/ERK signaling pathway facilitates VSVmediated oncolysis: implication for the defective interferon response in cancer cells. Mol Ther 15:1531-6.

157. Odenwald, W. F., H. Arnheiter, M. Dubois-Dalcq, and R. A. Lazzarini. 1986. Stereo images of vesicular stomatitis virus assembly. J Virol 57:922-32.

158. Ogden, J. R., R. Pal, and R. R. Wagner. 1986. Mapping regions of the matrix protein of vesicular stomatitis virus which bind to ribonucleocapsids, liposomes, and monoclonal antibodies. J Virol 58:860-8.

159. Okuma, K., E. Boritz, J. Walker, A. Sarkar, L. Alexander, and J. K. Rose. 2006. Recombinant vesicular stomatitis viruses encoding simian immunodeficiency virus receptors target infected cells and control infection. Virology 346:86-97.

160. Ono, K., M. E. Dubois-Dalcq, M. Schubert, and R. A. Lazzarini. 1987. A mutated membrane protein of vesicular stomatitis virus has an abnormal distribution within the infected cell and causes defective budding. J Virol 61:1332-41. 
161. Pak, C. C., A. Puri, and R. Blumenthal. 1997. Conformational changes and fusion activity of vesicular stomatitis virus glycoprotein: [125I]iodonaphthyl azide photolabeling studies in biological membranes. Biochemistry 36:8890-6.

162. Pal, R., Y. Barenholz, and R. R. Wagner. 1987. Vesicular stomatitis virus membrane proteins and their interactions with lipid bilayers. Biochim Biophys Acta 906:175-93.

163. Pal, R., B. W. Grinnell, R. M. Snyder, and R. R. Wagner. 1985. Regulation of viral transcription by the matrix protein of vesicular stomatitis virus probed by monoclonal antibodies and temperature-sensitive mutants. J Virol 56:386-94.

164. Panchal, R. G., G. Ruthel, T. A. Kenny, G. H. Kallstrom, D. Lane, S. S. Badie, L. Li, S. Bavari, and M. J. Aman. 2003. In vivo oligomerization and raft localization of Ebola virus protein VP40 during vesicular budding. Proc Natl Acad Sci U S A 100:15936-41.

165. Parent, L. J., R. P. Bennett, R. C. Craven, T. D. Nelle, N. K. Krishna, J. B. Bowzard, C. B. Wilson, B. A. Puffer, R. C. Montelaro, and J. W. Wills. 1995. Positionally independent and exchangeable late budding functions of the Rous sarcoma virus and human immunodeficiency virus Gag proteins. J Virol 69: 5455-60.

166. Perrault, J., and D. T. Kingsbury. 1974. Inhibitor of vesicular stomatitis virus transcriptase in purified virions. Nature 248:45-7.

167. Pestova, T. V., V. G. Kolupaeva, I. B. Lomakin, E. V. Pilipenko, I. N. Shatsky, V. I. Agol, and C. U. Hellen. 2001. Molecular mechanisms of translation initiation in eukaryotes. Proc Natl Acad Sci U S A 98:7029-36.

168. Petersen, J. M., L. S. Her, V. Varvel, E. Lund, and J. E. Dahlberg. 2000. The matrix protein of vesicular stomatitis virus inhibits nucleocytoplasmic transport when it is in the nucleus and associated with nuclear pore complexes. Mol Cell Biol 20:8590-601.

169. Pinto, L. H., L. J. Holsinger, and R. A. Lamb. 1992. Influenza virus M2 protein has ion channel activity. Cell 69:517-28.

170. Poirot, M. K., W. M. Schnitzlein, and M. E. Reichmann. 1985. The requirement of protein synthesis for VSV inhibition of host cell RNA synthesis. Virology 140:91-101.

171. Power, A. T., J. Wang, T. J. Falls, J. M. Paterson, K. A. Parato, B. D. Lichty, D. F. Stojdl, P. A. Forsyth, H. Atkins, and J. C. Bell. 2007. Carrier Cell-based Delivery of an Oncolytic Virus Circumvents Antiviral Immunity. Mol Ther 15:123-30.

172. Puri, A., J. Winick, R. J. Lowy, D. Covell, O. Eidelman, A. Walter, and R. Blumenthal. 1988. Activation of Vesicular Stomatitis-Virus Fusion with Cells by Pretreatment at Low Ph. Journal of Biological Chemistry 263:4749-4753.

173. Reidler, J. A., P. M. Keller, E. L. Elson, and J. Lenard. 1981. A fluorescence photobleaching study of vesicular stomatitis virus infected BHK cells. Modulation of $\mathrm{G}$ protein mobility by M protein. Biochemistry 20:1345-9.

174. Rigaut, K. D., D. E. Birk, and J. Lenard. 1991. Intracellular distribution of input vesicular stomatitis virus proteins after uncoating. J Virol 65:2622-8. 
175. Rinke, J., and J. A. Steitz. 1982. Precursor molecules of both human 5S ribosomal RNA and transfer RNAs are bound by a cellular protein reactive with anti-La lupus antibodies. Cell 29:149-59.

176. Robison, C. S., and M. A. Whitt. 2000. The membrane-proximal stem region of vesicular stomatitis virus $\mathrm{G}$ protein confers efficient virus assembly. J Virol 74:2239-46.

177. Roche, S., S. Bressanelli, F. A. Rey, and Y. Gaudin. 2006. Crystal structure of the low-pH form of the vesicular stomatitis virus glycoprotein G. Science 313:187-91.

178. Roche, S., F. A. Rey, Y. Gaudin, and S. Bressanelli. 2007. Structure of the prefusion form of the vesicular stomatitis virus glycoprotein G. Science 315: 843-8.

179. Romer-Oberdorfer, A., E. Mundt, T. Mebatsion, U. J. Buchholz, and T. C. Mettenleiter. 1999. Generation of recombinant lentogenic Newcastle disease virus from cDNA. Journal of General Virology 80:2987-2995.

180. Rose, J. K., L. Buonocore, and M. A. Whitt. 1991. A new cationic liposome reagent mediating nearly quantitative transfection of animal cells. Biotechniques 10:520-5.

181. Rosen, C. A., J. Siekierka, H. L. Ennis, and P. S. Cohen. 1984. Inhibition of protein synthesis in vesicular stomatitis virus infected Chinese hamster ovary cells: role of virus mRNA-ribonucleoprotein particle. Biochemistry 23:2407-11.

182. Sarkar, D. P., S. J. Morris, O. Eidelman, J. Zimmerberg, and R. Blumenthal. 1989. Initial stages of influenza hemagglutinin-induced cell fusion monitored simultaneously by two fluorescent events: cytoplasmic continuity and lipid mixing. J Cell Biol 109:113-22.

183. Schliwa, M., and G. Woehlke. 2003. Molecular motors. Nature 422:759-65.

184. Schnell, M. J., L. Buonocore, E. Kretzschmar, E. Johnson, and J. K. Rose. 1996. Foreign glycoproteins expressed from recombinant vesicular stomatitis viruses are incorporated efficiently into virus particles. Proc Natl Acad Sci U S A 93:11359-65.

185. Schnell, M. J., J. E. Johnson, L. Buonocore, and J. K. Rose. 1997. Construction of a novel virus that targets HIV-1-infected cells and controls HIV-1 infection. Cell 90:849-57.

186. Schubert, M., J. D. Keene, R. C. Herman, and R. A. Lazzarini. 1980. Site on the vesicular stomatitis virus genome specifying polyadenylation and the end of the L gene mRNA. J Virol 34:550-9.

187. Seth, R. B., L. Sun, and Z. J. Chen. 2006. Antiviral innate immunity pathways. Cell Res 16:141-7.

188. Shinozaki, K., O. Ebert, C. Kournioti, Y. S. Tai, and S. L. Woo. 2004. Oncolysis of multifocal hepatocellular carcinoma in the rat liver by hepatic artery infusion of vesicular stomatitis virus. Mol Ther 9:368-76.

189. Sieczkarski, S. B., and G. R. Whittaker. 2003. Differential requirements of Rab5 and Rab7 for endocytosis of influenza and other enveloped viruses. Traffic 4:333-43. 
190. Simon, K. O., P. A. Whitaker-Dowling, J. S. Youngner, and C. C. Widnell. 1990. Sequential disassembly of the cytoskeleton in BHK21 cells infected with vesicular stomatitis virus. Virology 177:289-97.

191. Stallknecht, D. E. 2000. VSV-NJ on Ossabaw Island, Georgia - The truth is out there. Tropical Veterinary Diseases 916:431-436.

192. Stojdl, D. F., B. Lichty, S. Knowles, R. Marius, H. Atkins, N. Sonenberg, and J. C. Bell. 2000. Exploiting tumor-specific defects in the interferon pathway with a previously unknown oncolytic virus. Nat Med 6:821-5.

193. StojdI, D. F., B. D. Lichty, B. R. tenOever, J. M. Paterson, A. T. Power, S. Knowles, R. Marius, J. Reynard, L. Poliquin, H. Atkins, E. G. Brown, R. K. Durbin, J. E. Durbin, J. Hiscott, and J. C. Bell. 2003. VSV strains with defects in their ability to shutdown innate immunity are potent systemic anti-cancer agents. Cancer Cell 4:263-75.

194. Stuchell, M. D., J. E. Garrus, B. Muller, K. M. Stray, S. Ghaffarian, R. McKinnon, H. G. Krausslich, S. G. Morham, and W. I. Sundquist. 2004. The human endosomal sorting complex required for transport (ESCRT-I) and its role in HIV-1 budding. J Biol Chem 279:36059-71.

195. Swinteck, B. D., and D. S. Lyles. 2008. Plasma Membrane Microdomains Containing Vesicular Stomatitis Virus M Protein Are Separate from Microdomains Containing G Protein and Nucleocapsids. J Virol 82:5536-47.

196. Terenzi, F., M. J. deVeer, H. Ying, N. P. Restifo, B. R. Williams, and R. H. Silverman. 1999. The antiviral enzymes PKR and RNase L suppress gene expression from viral and non-viral based vectors. Nucleic Acids Res 27:4369-75.

197. Thacore, H. R. 1978. Effect of interferon on transcription and translation of vesicular stomatitis virus in human and simian cell cultures. J Gen Virol 41: 421-6.

198. Thimmig, R. L., J. V. Hughes, R. J. Kinders, A. G. Milenkovic, and T. C. Johnson. 1980. Isolation of the glycoprotein of vesicular stomatitis virus and its binding to cell surfaces. J Gen Virol 50:279-91.

199. Thomas, D., W. W. Newcomb, J. C. Brown, J. S. Wall, J. F. Hainfeld, B. L. Trus, and A. C. Steven. 1985. Mass and molecular composition of vesicular stomatitis virus: a scanning transmission electron microscopy analysis. J Virol 54:598-607.

200. Timmins, J., R. W. Ruigrok, and W. Weissenhorn. 2004. Structural studies on the Ebola virus matrix protein VP40 indicate that matrix proteins of enveloped RNA viruses are analogues but not homologues. FEMS Microbiol Lett 233: 179-86.

201. Timmins, J., G. Schoehn, S. Ricard-Blum, S. Scianimanico, T. Vernet, R. W. Ruigrok, and W. Weissenhorn. 2003. Ebola virus matrix protein VP40 interaction with human cellular factors Tsg101 and Nedd4. J Mol Biol 326: 493-502.

202. van den Pol, A. N., K. P. Dalton, and J. K. Rose. 2002. Relative neurotropism of a recombinant rhabdovirus expressing a green fluorescent envelope glycoprotein. J Virol 76:1309-27. 
203. Villarreal, L. P., M. Breindl, and J. J. Holland. 1976. Determination of molar ratios of vesicular stomatitis virus induced RNA species in BHK21 cells. Biochemistry 15:1663-7.

204. von Kobbe, C., J. M. van Deursen, J. P. Rodrigues, D. Sitterlin, A. Bachi, X. Wu, M. Wilm, M. Carmo-Fonseca, and E. Izaurralde. 2000. Vesicular stomatitis virus matrix protein inhibits host cell gene expression by targeting the nucleoporin Nup98. Mol Cell 6:1243-52.

205. Webster, R. G., W. J. Bean, O. T. Gorman, T. M. Chambers, and Y. Kawaoka. 1992. Evolution and ecology of influenza A viruses. Microbiol Rev 56:152-79.

206. Weck, P. K., and R. R. Wagner. 1979. Transcription of vesicular stomatitis virus is required to shut off cellular RNA synthesis. J Virol 30:410-3.

207. White, J., K. Matlin, and A. Helenius. 1981. Cell fusion by Semliki Forest, influenza, and vesicular stomatitis viruses. J Cell Biol 89:674-9.

208. Whitlow, Z. W., J. H. Connor, and D. S. Lyles. 2008. New mRNAs are preferentially translated during vesicular stomatitis virus infection. Journal of Virology 82:2286-2294.

209. Whitlow, Z. W., J. H. Connor, and D. S. Lyles. 2006. Preferential translation of vesicular stomatitis virus mRNAs is conferred by transcription from the viral genome. J Virol 80:11733-42.

210. Whitt, M. A., L. Chong, and J. K. Rose. 1989. Glycoprotein cytoplasmic domain sequences required for rescue of a vesicular stomatitis virus glycoprotein mutant. J Virol 63:3569-78.

211. Whitt, M. A., P. Zagouras, B. Crise, and J. K. Rose. 1990. A fusion-defective mutant of the vesicular stomatitis virus glycoprotein. J Virol 64:4907-13.

212. Wilson, T., and J. Lenard. 1981. Interaction of wild-type and mutant M protein vesicular stomatitis virus with nucleocapsids in vitro. Biochemistry 20:1349-54.

213. Wollmann, G., M. D. Robek, and A. N. van den Pol. 2007. Variable deficiencies in the interferon response enhance susceptibility to vesicular stomatitis virus oncolytic actions in glioblastoma cells but not in normal human glial cells. J Virol 81:1479-91.

214. Wollmann, G., P. Tattersall, and A. N. van den Pol. 2005. Targeting human glioblastoma cells: comparison of nine viruses with oncolytic potential. J Virol 79:6005-22.

215. Wong, R. W., G. Blobel, and E. Coutavas. 2006. Rae1 interaction with NuMA is required for bipolar spindle formation. Proc Natl Acad Sci U S A 103:19783-7.

216. Yasuda, J., and E. Hunter. 1998. A proline-rich motif (PPPY) in the Gag polyprotein of Mason-Pfizer monkey virus plays a maturation-independent role in virion release. J Virol 72:4095-103.

217. Ye, Z., W. Sun, K. Suryanarayana, P. Justice, D. Robinson, and R. R. Wagner. 1994. Membrane-binding domains and cytopathogenesis of the matrix protein of vesicular stomatitis virus. J Virol 68:7386-96.

218. Yuan, H., S. Puckett, and D. S. Lyles. 2001. Inhibition of host transcription by vesicular stomatitis virus involves a novel mechanism that is independent of phosphorylation of TATA-binding protein (TBP) or association of TBP with TBP-associated factor subunits. J Virol 75:4453-8. 
219. Yuan, H., B. K. Yoza, and D. S. Lyles. 1998. Inhibition of host RNA polymerase II-dependent transcription by vesicular stomatitis virus results from inactivation of TFIID. Virology 251:383-92.

220. Zakowski, J. J., W. A. Petri, Jr., and R. R. Wagner. 1981. Role of matrix protein in assembling the membrane of vesicular stomatitis virus: reconstitution of matrix protein with negatively charged phospholipid vesicles. Biochemistry 20:3902-7.

221. Zakowski, J. J., and R. R. Wagner. 1980. Localization of membrane-associated proteins in vesicular stomatitis virus by use of hydrophobic membrane probes and cross-linking reagents. J Virol 36:93-102.

222. Zhirnov, O. P. 1990. Solubilization of matrix protein M1/M from virions occurs at different $\mathrm{pH}$ for orthomyxo- and paramyxoviruses. Virology 176:274-9. 


\section{Vita}

Chad Edward Mire was born on February 26, 1977 in Baton Rouge, Louisiana. He graduated from Clearview Regional High School in Mullica Hill, New Jersey in the spring of 1995 and after graduation enrolled at Gettysburg College where he studied biology and played football from the fall of 1995 to the winter of 1998. After taking a self-imposed break from his studies, he enrolled at Rowan Universiy in the fall of 2000 and graduated with a B.S. in biology in the spring of 2002. Following his undergraduate studies, he studied at the University of Tennessee Health Science Center in Memphis, Tennessee in the lab of Michael A. Whitt, Ph.D. and successfully defended his dissertation on May 23, 2008. After graduate school, he went on to do his postdoctoral work in the lab of Thomas Geisbert, Ph.D. at Boston University in the National Emerging Infectious Diseases Laboratroy (NEIDL) where he studied pathogenesis of emerging infectious diseases and rational countermeasures. 